\title{
Advancement of
}

\section{Measurement Techniques for}

\section{Tunable Resistive Pulse}

\section{Sensing}

by

Eva Weatherall

A thesis

submitted to the Victoria University of Wellington

in fulfilment of the

requirements for the degree of

Doctor of Philosophy

in Chemistry.

Victoria University of Wellington

2017 



\begin{abstract}
Tunable resistive pulse sensing (TRPS) is a particle-by-particle analysis technique combining the Coulter principle with size-tunable pores. TRPS can be used to characterize biological and synthetic particles $50 \mathrm{~nm}-20$ $\mu \mathrm{m}$ in diameter. Information is obtained from the resistive pulse signal, a transient change in ionic current observed when a particle passes through the pore. TRPS has been shown to provide excellent resolution and accuracy for measuring particle size and concentration as well as providing information about particle charge. TRPS is therefore applicable to many industrial and fundamental research areas involving aptamers, drug delivery particles, extracellular vesicles and other biological particle types. Advancement of this technology requires a better understanding of the technique, particularly in the area of particle surface charge measurement and this Thesis helps to provide that understanding.
\end{abstract}

In this work, firstly particle $\zeta$-potential measurement using TRPS was 
investigated. A number of different measurement methods are presented and the uncertainties associated with each method are outlined. The $\zeta-$ potential for a variety of particles with different surface charges were measured in a range of electrolytes.

Particle $\zeta$-potential measurements were then improved upon with the addition of streaming potential measurements to measure the pore surface charge. The $\zeta$-potential of the pore surface, which makes a significant contribution to particle $\zeta$-potential calculations, was measured using a set up which works alongside the $q$ Nano. Streaming potential measurements were also used to investigate changes in the pore surface charge following application of number of different chemical coatings. The volume of data collected and detail of analysis in this work (including uncertainties) is unprecedented in TRPS $\zeta$-potential measurements.

Biphasic pulses arising from the charge on the particles were investigated. The pulse is conventionally resistive, but biphasic pulses which include both resistive and conductive components are significant for $<50$ $\mathrm{mM}$ salt concentrations when measuring $200 \mathrm{~nm}$ particles. The experimental variables investigated include the concentration of the electrolyte, particle charge, pore size, applied voltage, and the direction of particle 
motion. Conductive pulse size was seen to decrease with increasing electrolyte concentration and pore size and increase with applied voltage. A linear relationship was found between conductive pulse magnitude and particle surface group density. The influence of direction of motion on conductive pulses was consistent with concentration polarization of an ion selective pore. Biphasic pulses were also seen to affect conventional TRPS particle size measurements.

Finally, size distribution broadening due to varying particle trajectories was investigated. Pulse size distributions for monodisperse particles became broader when the pore size was increased and featured two distinct peaks. Relatively large pulses are produced by particles with trajectories passing near to the edge of the pore. Other experiments determined that pulse size distributions are independent of applied voltage but broaden with increasing pressure applied across the membrane. 


\section{Acknowledgments}

Thanks to my supervisor Dr. Geoff Willmott for his knowledge and guidance. Your enthusiasm and dedication to this project were great motivators. I'm extremely grateful for your support. Thanks also to my secondary supervisor Dr. Justin Hodgkiss for your help and support.

Thanks to my family, my grandparents who I wish could see me graduate, my Mum and Dad, my aunt Ann, and my siblings Ruth and Paul for your love and encouragement. Thanks to my partner Angus and his family for their support. To Claire, Elly, and Marah for their friendship and advice.

Thanks to Elf for his help with python programming and SEM. Thanks to Joseph Deere at Callaghan Innovation for his help in the lab and his interest in my research. Also to Mike, Andrea and John in the Sensing and 
Automation group at Callaghan Innovation. Thanks to Ben, Rebecca, Nick and Nadiah at Izon Science for the hospitality and knowledge sharing.

Thanks to the MacDiarmid Institute, the Royal Society of New Zealand, the Victoria University Submission Scholarship and Victoria Clubs sponsorship for funding. 


\section{List of Symbols}

\begin{tabular}{|c|c|c|}
\hline Symbol & Units & Description \\
\hline$\alpha$ & & $\begin{array}{l}\text { macroscopic strain, } \alpha=(X- \\
\left.X_{0}\right) / X_{0}\end{array}$ \\
\hline$\varepsilon$ & $\mathrm{F} \mathrm{m}^{-1}$ & absolute permittivity, $\varepsilon=\varepsilon_{0} \varepsilon_{r}$ \\
\hline$\zeta$ & $\mathrm{V}$ & zeta potential \\
\hline$\zeta_{C a l}$ & $\mathrm{~V}$ & $\begin{array}{l}\zeta \text {-potential of calibration stan- } \\
\text { dard particle }\end{array}$ \\
\hline$\zeta_{n e t}$ & $\mathrm{~V}$ & $\zeta_{\text {particle }}-\zeta_{\text {pore }}$ \\
\hline$\zeta_{\text {particle }}$ & $\mathrm{V}$ & $\zeta$-potential of particle \\
\hline$\zeta_{p o r e}$ & $\mathrm{~V}$ & $\zeta$-potential of pore walls \\
\hline$\zeta_{\text {Sample }}$ & $\mathrm{V}$ & $\zeta$-potential of sample particle \\
\hline$\eta$ & Pa s & fluid dynamic viscosity \\
\hline$\kappa$ & $\mathrm{m}^{-1}$ & inverse of the Debye length \\
\hline
\end{tabular}


viii

\begin{tabular}{|c|c|c|}
\hline$\lambda$ & $\mathrm{S} \mathrm{m}^{-1}$ & electrolyte conductivity \\
\hline$\mu_{e o}$ & $\mathrm{~m}^{2} \mathrm{~V}^{-1} \mathrm{~s}^{-1}$ & electroosmotic mobility \\
\hline$\mu_{e p h}$ & $\mathrm{~m}^{2} \mathrm{~V}^{-1} \mathrm{~s}^{-1}$ & electrophoretic mobility \\
\hline$\rho$ & $\Omega \mathrm{m}$ & electrolyte resistivity \\
\hline$\rho_{e}$ & $\mathrm{C} \mathrm{mol} \mathrm{L}^{-1}$ & excess surface charge \\
\hline$\sigma$ & $\mathrm{Cm}^{-2}$ & surface charge density \\
\hline$\sigma_{n}^{p d f}$ & $\mathrm{~m} \mathrm{~s}^{-1}$ & uncertainty in $v_{n}^{p d f}$ \\
\hline$\Delta \Phi$ & $\mathrm{V}$ & streaming potential \\
\hline$\Psi$ & $\mathrm{V}$ & electrostatic field potential \\
\hline$\Psi_{0}$ & $\mathrm{~V}$ & $\begin{array}{l}\text { electrostatic field potential at } \\
\text { surface }\end{array}$ \\
\hline$\Psi_{s t}$ & $\mathrm{~V}$ & $\begin{array}{l}\text { electrostatic field potential at the } \\
\text { Stern layer }\end{array}$ \\
\hline$a$ & $\mathrm{~m}$ & radius of small pore opening \\
\hline$A$ & $\mathrm{~m}^{2}$ & pore cross-sectional area \\
\hline$b$ & $\mathrm{~m}$ & radius of large pore opening \\
\hline$C$ & $\mathrm{~mol} \mathrm{~L}^{-1}$ & concentration \\
\hline$c_{i}$ & $\mathrm{~mol} \mathrm{~L}^{-1}$ & concentration of ion $i$ in solution \\
\hline$d$ & $\mathrm{~m}$ & particle diameter \\
\hline
\end{tabular}




\begin{tabular}{|c|c|c|}
\hline$D$ & $\mathrm{~m}$ & cylindrical pore diameter \\
\hline$D_{p d f / e p}$ & & $\begin{array}{l}\text { dimensionless ratio showing the } \\
\text { relative importance of pressure- } \\
\text { driven flow to electrophoresis }\end{array}$ \\
\hline$e$ & $\mathrm{C}$ & charge of an electron \\
\hline $\mathbf{E}$ & $\mathrm{V} \mathrm{m}^{-1}$ & electric field \\
\hline$g$ & $\mathrm{~s}^{-1} \mathrm{~Pa}^{-1}$ & $\begin{array}{l}\text { gradient of pressure against par- } \\
\text { ticle rate }\end{array}$ \\
\hline$I$ & A & current \\
\hline$I_{0}$ & $\mathrm{~A}$ & $\begin{array}{l}\text { baseline current through the } \\
\text { aperture }\end{array}$ \\
\hline$I_{c}$ & A & conduction current \\
\hline$I_{n}$ & $\mathrm{~A}$ & $\begin{array}{l}\text { current at } n \text { fraction of the block- } \\
\text { ade magnitude }\end{array}$ \\
\hline$I_{s}$ & A & streaming current \\
\hline$I S$ & $\operatorname{mol~L}{ }^{-1}$ & ionic strength \\
\hline$\Delta I$ or $\Delta I_{r}$ & A & resistive pulse magnitude \\
\hline$\Delta I_{c}$ & $\mathrm{~A}$ & conductive pulse magnitude \\
\hline
\end{tabular}




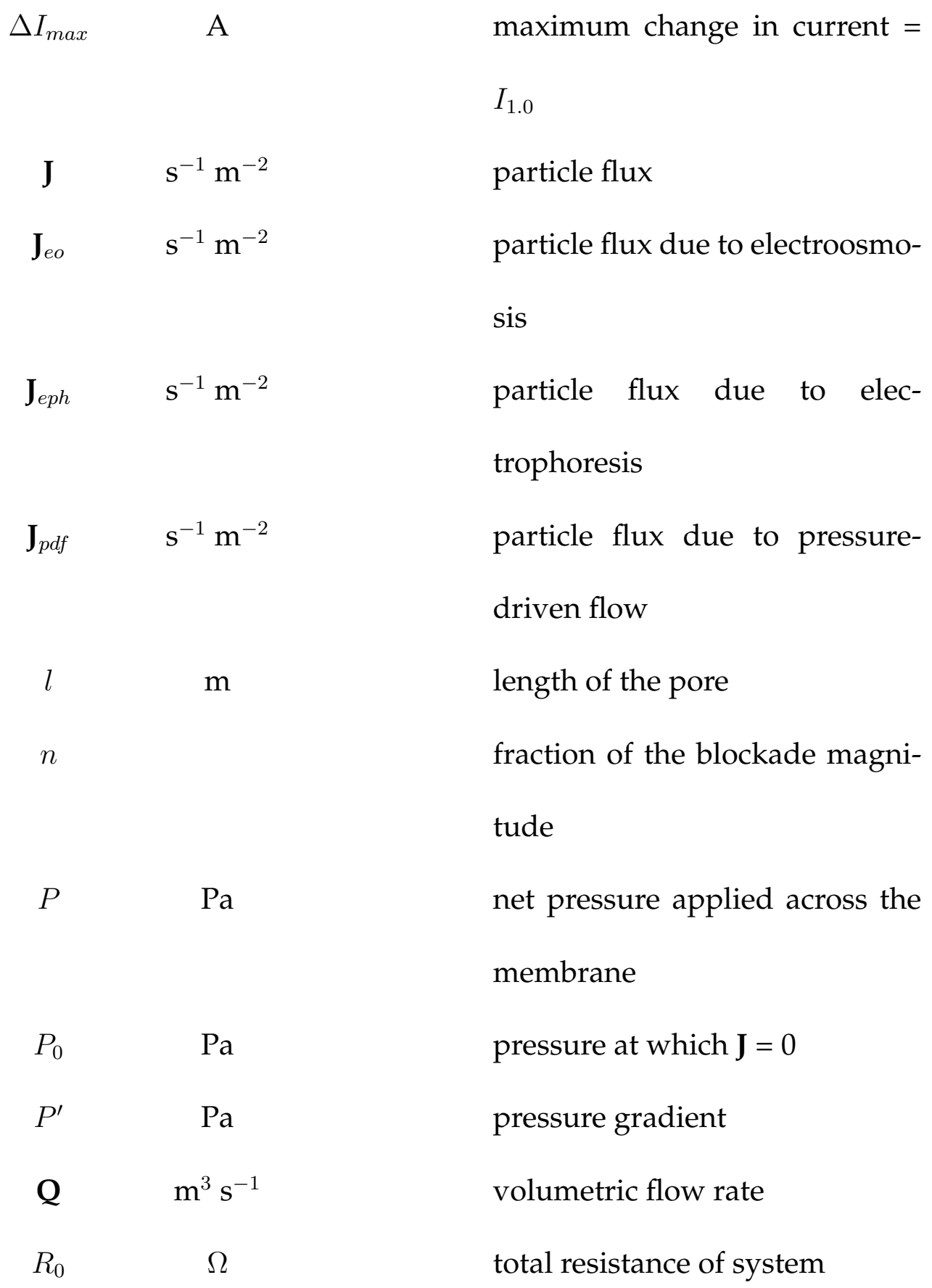




\begin{tabular}{|c|c|c|}
\hline$R_{\text {end }}$ & $\Omega$ & $\begin{array}{l}\text { electrical resistance due to pore } \\
\text { openings }\end{array}$ \\
\hline$R_{f}$ & & rectification ratio \\
\hline$\Delta R$ & $\Omega$ & $\begin{array}{l}\text { increase in resistance of system } \\
\text { due to presence of particle }\end{array}$ \\
\hline$T_{n}$ & $\mathrm{~s}$ & time at $n$ \\
\hline$v$ & $\mathrm{~m} \mathrm{~s}^{-1}$ & fluid velocity \\
\hline$v_{n}$ & $\mathrm{~m} \mathrm{~s}^{-1}$ & $1 / T_{n}$ \\
\hline$v^{e o}$ & $\mathrm{~m} \mathrm{~s}^{-1}$ & electroosmotic fluid velocity \\
\hline$v^{e l}$ & $\mathrm{~m} \mathrm{~s}^{-1}$ & electrokinetic fluid velocity \\
\hline$v^{e p h}$ & $\mathrm{~m} \mathrm{~s}^{-1}$ & electrophoretic fluid velocity \\
\hline$v^{p d f}$ & $\mathrm{~m} \mathrm{~s}^{-1}$ & $\begin{array}{l}\text { pressure-driven flow fluid ve- } \\
\text { locity }\end{array}$ \\
\hline$v^{t o t}$ & $\mathrm{~m} \mathrm{~s}^{-1}$ & total fluid velocity \\
\hline$V_{0}$ & $\mathrm{~V}$ & applied voltage \\
\hline$X$ & $\mathrm{~m}$ & membrane stretch \\
\hline$X_{0}$ & $\mathrm{~m}$ & $X$ with no stress applied \\
\hline$y$ & $\mathrm{~m}$ & $\begin{array}{l}\text { distance from surface into fluid } \\
\text { through EDL }\end{array}$ \\
\hline
\end{tabular}


xii

$y_{s t}$

$\mathrm{m}$

$y_{\zeta}$

m

$z$

$\mathrm{m}$

$z_{i}$ distance from surface to the

Stern layer

distance from surface to the

shear plane

distance into pore from small

pore opening

ion charge magnitude of ion $i$ in

solution 


\title{
List of Abbreviations
}

\author{
Abbreviation \\ Description \\ $\alpha-\mathrm{HL}$ \\ $\alpha$-hemolysin \\ ABA \\ 3-aminobenzylamine \\ Afp \\ Anti-feeding prophage \\ ALE \\ Arbitrary Lagrangian Euler \\ ATR \\ Attenuated total reflectance \\ bp \\ Base pairs \\ C. Parvum \\ Cryptosporidium paroum \\ CPC \\ Carboxylated polystyrene cali- \\ bration (particles) \\ CPC\#\#\# \\ CPC particles of nominal diam- \\ eter \#\#\#
}

xiii 
xiv

\begin{tabular}{|c|c|}
\hline $\mathrm{CPN}$ & $\begin{array}{l}\text { Non-carboxylated polystyrene } \\
\text { calibration (particles) }\end{array}$ \\
\hline CPN\#\#\# & $\begin{array}{l}\text { CPN particles of nominal diam- } \\
\text { eter \#\#\# }\end{array}$ \\
\hline $\mathrm{CV}$ & Coefficient of variance \\
\hline DCS & Disc centrifugal sedimentation \\
\hline DFCC & Disposable folded capillary cell \\
\hline DLS & Dynamic light scattering \\
\hline DP & Dexamthasone phosphate \\
\hline ds & Double stranded \\
\hline E. coli & Escherichia coli \\
\hline EDL & Electrical double layer \\
\hline EDTA & Ethylenediaminetetraacetic acid \\
\hline EM & Electron microscopy \\
\hline EV & Extracellular vesicles \\
\hline FC & Flow cytometry \\
\hline FEM & Finite element modelling \\
\hline FFF & Field flow fractionation \\
\hline
\end{tabular}




\begin{tabular}{|c|c|}
\hline FTIR & $\begin{array}{l}\text { Fourier transform infrared spec- } \\
\text { troscopy }\end{array}$ \\
\hline FWHM & Full-width half-maximum \\
\hline HEPES & $\begin{array}{l}\text { 4-2-hydroxyethyl-1- } \\
\text { piperazineethanesulfonic acid }\end{array}$ \\
\hline ICR & Ion-current rectification \\
\hline ICS & Izon control suite \\
\hline $\operatorname{IgG}$ & Immunoglobulin $\mathrm{G}$ \\
\hline miRNA & microRNA \\
\hline MSC & Mesenchymal stem cells \\
\hline $\mathrm{NO}$ & Nitric oxide \\
\hline NP\#\#\# & $\begin{array}{l}\text { Nanopore suited for measuring } \\
\text { particles with nominal diameter } \\
\# \# \#\end{array}$ \\
\hline NTA & Nanoparticle tracking analysis \\
\hline PAA & Polyacrylic acid \\
\hline PBS & Phosphate buffered saline \\
\hline PDGF & Platelet-derived growth factor \\
\hline PEEK & Polyetheretherketone \\
\hline
\end{tabular}


xvi

\begin{tabular}{|c|c|}
\hline PEG & Polyethylene glycol \\
\hline PEI & Polyethylenimine \\
\hline PHEMA & Polyhydroxyethylmethacrylate \\
\hline PLGA & Poly(lactic-co-glycolic acid) \\
\hline PMP & Platelet microparticle \\
\hline PNA & Peptide nucleic acid \\
\hline PSNE & Phase-shift nanoemulsion \\
\hline PTA & $\begin{array}{l}\text { Particle tracking analysis } \\
\text { (Equivalent to NTA) }\end{array}$ \\
\hline $\mathrm{RBC}$ & Red blood cell \\
\hline RMS & Root mean squared \\
\hline RPS & Resistive pulse sensing \\
\hline S. aureus & Staphylococcus aureus \\
\hline SEB & Standard electrolyte buffer \\
\hline SEM & Scanning electron microscopy \\
\hline SMA & SubMiniature version A \\
\hline $\mathrm{SPB}$ & Superparamagnetic bead \\
\hline SPC & Soy phosphatidylcholin \\
\hline
\end{tabular}




$\begin{array}{cl}\text { SPION } & \text { Superparamagnetic iron-oxide } \\ & \text { nanoparticle } \\ \text { sS } & \text { Single strand } \\ \text { TEM } & \text { Transmission electron mi- } \\ & \text { croscopy } \\ \text { TPU } & \text { Thermoplastic polyurethane } \\ \text { tris } & \text { tris(hydroxymethyl)aminomethane } \\ \text { TRPS } & \text { Tunable resistive pulse sensing } \\ \text { UDC } & \text { Universal dip cell } \\ \text { VPM } & \text { Variable pressure manometer } \\ \text { XPS } & \text { X-ray photoelectron spec- } \\ & \text { troscopy }\end{array}$




\section{Contents}

1 Introduction 1

1.1 Particle Characterization . . . . . . . . . . . . 1

1.2 Early Resistive Pulse Sensing . . . . . . . . . . . . 5

1.3 Introduction to Tunable Resistive Pulse Sensing $\ldots . . . .9$

1.4 Thesis Structure . . . . . . . . . . . . . . . . . . . 12

2 Literature Review 13

2.1 Technical Aspects . . . . . . . . . . . . . . . . . 15

2.1.1 Pore Specimens and Stretching . . . . . . . . . . 15 
2.1 .2 Apparatus .................... 18

2.1.3 Analysis and Measurement Protocols . . . . . . . . 22

2.1.4 Comparison with Other Techniques . . . . . . . 38

2.1.5 Distinct Characteristics of TRPS . . . . . . . 43

2.2 Applications .................... 46

2.2.1 Diagnostics and Genomics . . . . . . . . . 46

2.2.2 Extracellular Vesicles . . . . . . . . . . . . 57

2.2.3 Nanomedicine ................ 65

2.2.4 Phages, Viruses, Bacteria and Algae . . . . . . . 73

2.2 .5 Other ...................... 77

2.3 Conclusion ..................... 81

3 Materials and Methods $\quad 85$

3.1 Materials ..................... 86

3.1.1 Electrolytes ................. 86 


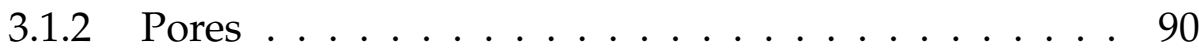

3.1.3 Particles ....................... 92

3.2 Experimental Equipment . . . . . . . . . . . . . . 93

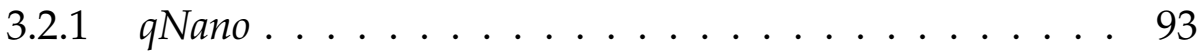

$3.2 .2 \mathrm{VPM} \ldots \ldots \ldots \ldots \ldots$

3.2.3 Pulse Analysis . . . . . . . . . . . . . . . . . . . 94

3.2.4 Zetasizer ..................... 96

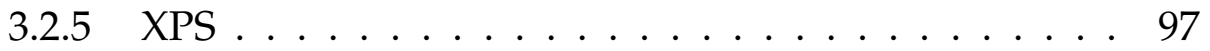

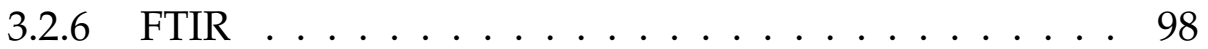

3.2 .7 SEM . . . . . . . . . . . . . . 98

3.3 Pore Coating Methods . . . . . . . . . . . . . . . . . . . . 101

3.3.1 Dopamine and 'Fe + Dopamine' . . . . . . . . . . . 101

$3.3 .2 \quad$ PEI/PAA ....................... 101 
4.1 Theory and Analysis . . . . . . . . . . . . . 105

4.1.1 Variable Pressure Method . . . . . . . . . . . . . 111

4.1.2 Velocity Profile Method and Method I . . . . . . . . . 113

4.1.3 TRPS Calibration Charge Methods (Methods II-IV) . 116

4.1.4 Measurement Uncertainties . . . . . . . . . . . . 126

4.2 Results and Discussion . . . . . . . . . . . . . . . 128

4.2.1 Comparing Method I and Method II . . . . . . . . . 128

4.2.2 DLS Measurements . . . . . . . . . . . . . . 135

4.2.3 Method III and IV with Varied Electrolytes . . . . . . 140

4.2.4 Methods III and IV with Varied $\mathrm{pH} \ldots . . . . . .147$

4.3 Conclusion . . . . . . . . . . . . . . . . . . 151

5 Improved $\zeta$-potential Measurements

5.1 Streaming Potential Theory . . . . . . . . . . . . 156

5.2 Streaming Potential Measurement Set-Up . . . . . . . . . 158 
$5.3 \zeta_{\text {particle }}$ and $\zeta_{\text {pore }}$ Measurements . . . . . . . . . . . 161

5.3 .1 Pore Size . . . . . . . . . . . . . . . . 161

5.3.2 Electrolyte Concentration . . . . . . . . . . . 165

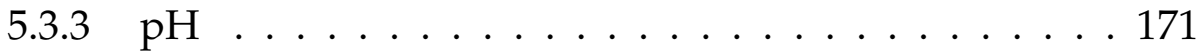

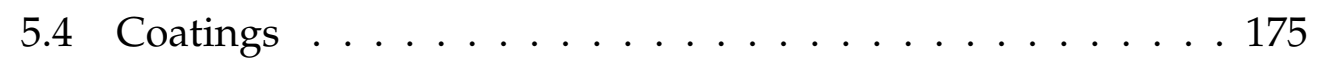

5.4 .1 Dopamine .................. 176

5.4.2 Polymer Brush Functionalisation . . . . . . . . . . 192

$5.4 .3 \quad$ PEI/PAA . . . . . . . . . . . . . . 194

5.5 Oscillations . . . . . . . . . . . . . . . . 198

5.6 Conclusion . . . . . . . . . . . . . . . . . 202

6 Biphasic Pulses 205

6.1 Introduction . . . . . . . . . . . . . . . 205

6.2 Background ..................... 206

6.3 Results and Discussion . . . . . . . . . . . . . . . 209 
6.3.1 Particle Direction, Applied Bias and Pore Orientation 210

6.3.2 Particle Charge . . . . . . . . . . . . . 217

6.3.3 Electrolyte Concentration . . . . . . . . . . . 220

6.3.4 Applied Voltage . . . . . . . . . . . . . . 224

6.3 .5 Applied Stretch . . . . . . . . . . . . . 227

6.3.6 Particle Sizing . . . . . . . . . . . . . . . 229

6.4 Conclusion . . . . . . . . . . . . . . . 231

7 Broadening of Pulse Size Distributions 233

7.1 Reference Data . . . . . . . . . . . . . . 238

7.1.1 Finite Element Modelling of Particle Trajectories . . . 238

7.1.2 Single Particle Pressure Reversal . . . . . . . . . . 242

7.2 Results and Discussion . . . . . . . . . . . . . . 245

7.2.1 Stretch Variation . . . . . . . . . . . 246

7.2.2 Pressure Variation . . . . . . . . . . . 252 
7.2.3 Pore Orientation and Applied Potential . . . . . . . . 257

7.2.4 Electrokinetic Effects . . . . . . . . . . . . . . 259

7.2 .5 Discussion . . . . . . . . . . . . . 262

7.3 Conclusion . . . . . . . . . . . . . . . . 263

8 Conclusions and Future Work

$8.1 \zeta$-potential Measurements . . . . . . . . . . 266

8.2 Biphasic Pulses . . . . . . . . . . . . . . . 271

8.3 Distribution Broadening . . . . . . . . . . . . 272

8.4 Future work . . . . . . . . . . . . . . . . . . 274

$\begin{array}{ll}\text { Bibliography } & 275\end{array}$

$\begin{array}{ll}\text { Appendices } & 307\end{array}$

A Publications and Presentations . . . . . . . . . . . . . 309

B SEM Images $\ldots \ldots . \ldots 313$ 
C $\quad \zeta$-potential Raw Data Example: Method IV . . . . . . . . . 323 


\section{List of Figures}

1.1 Schematic section through a tunable pore. . . . . . . . . . 4

1.2 Sketch of the original nanopore sequencing concept. . . . . . 7

2.1 Specimen imaging. . . . . . . . . . . . . 16

2.2 The $q$ Nano TRPS apparatus. . . . . . . . . . . . . . 20

2.3 Size distributions for urinary EVs. . . . . . . . . . . . 32

2.4 Pulses from an experiment and semi-analytic model. . . . . 34

2.5 Particle sets distinguished from a mixture. . . . . . . . . . 36

2.6 Comparison of TRPS with other characterization techniques. 40

2.7 TRPS experiments using DNA. . . . . . . . . . . . . . 48 xxvii 
2.8 Nanorod aggregation. . . . . . . . . . . . . 53

2.9 Size histograms for nanoparticles coupled to aptamers. . . . 55

2.10 TRPS experiments using EVs. . . . . . . . . . . . . . 59

$2.11 \mathrm{pH}$ responsive expansile nanoparticles. . . . . . . . . . . 70

2.12 TRPS of a phage and a virus. . . . . . . . . . 74

2.13 Histogram of published papers each year for TRPS. . . . . . 82

3.1 Example of SEM to size particles. . . . . . . . . . . . . 99

3.2 Example of SEM of pores . . . . . . . . . . . . 100

4.1 EDL diagram. . . . . . . . . . . . . . . 106

4.2 S-curves from variable pressure method. . . . . . . . . . . 111

4.3 Velocity profile plot. . . . . . . . . . . . . . . 114

4.4 Schematic of the multi-point charge measurement model. . . 117

4.5 Schematic $1 / T_{n}$ against $V$ calibration plot. . . . . . . . . 120

4.6 Schematic $1 / T_{n}$ against $P$ calibration plot. . . . . . . . 122 
4.7 SEM of tungsten needles. . . . . . . . . . . . . . . 128

$4.8 \zeta$-potential for charged and uncharged particles. . . . . . . . 129

4.9 Schematics of DLS measurement cells. . . . . . . . . . . . . 136

4.10 Plots showing particle charge using UDC and DFCCs. . . . . 138

$4.11 \zeta_{\text {particle }}$ in 3 electrolytes. . . . . . . . . . . . . . . 141

$4.12 \zeta_{\text {particle }}$ in 11 electrolyte concentrations. . . . . . . . . . . . 142

$4.13 \zeta$-potential of particle sets at different pHs . . . . . . . . . 149

4.14 Measurement difficulty with and without surfactant. . . . . 150

5.1 Schematic of TRPS streaming potential measurement set-up. 159

5.2 Example of streaming potential plot. . . . . . . . . . . . 160

$5.3 \zeta_{\text {pore }}$ measurements with stretch $\ldots \ldots . \ldots . \ldots 162$

$5.4 \zeta_{\text {pore }}$ and $\zeta_{\text {particle }}$ at different stretches . . . . . . . . . . 164

$5.5 \zeta_{\text {pore }}$ for an uncoated NP150 pore at 3 electrolyte concentra-

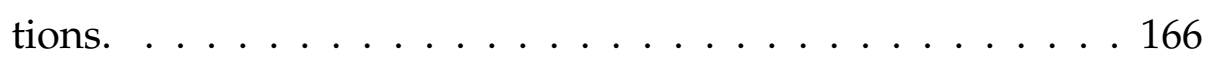


$5.6 \zeta_{\text {particle }}$ of particles in 3 PBS concentrations. . . . . . . . 168

$5.7 \zeta_{\text {pore }}$ at a range of $\mathrm{pHs}$ to identify isoelectic point. . . . . . . 172

$5.8 \zeta_{\text {particle }}$ measurement of 8 particle types at 4 different $\mathrm{pHs} . \quad$. 173

5.9 FTIR transmittance spectra. . . . . . . . . . . . . . 179

5.10 XPS surveys. . . . . . . . . . . . . . . . 181

5.11 High resolution XPS $(\mathrm{O} 1 \mathrm{~s}$ and $\mathrm{C} 1 \mathrm{~s}) \ldots \ldots . \ldots . . \ldots 184$

5.12 High resolution XPS (N 1s). . . . . . . . . . . . 185

5.13 Schematic of ICR $I-V$ curves. . . . . . . . . . . 188

5.14 Ratchet model . . . . . . . . . . . . . . . . . . . . . 189

5.15 ICR before and after dopamine coating. . . . . . . . . . . 190

5.16 ICR before and after dopamine $\left(\mathrm{Fe}^{2+}\right)$ coating. . . . . . . . . 191

5.17 Change in $\zeta_{\text {pore }}$ for polymer brush coatings. . . . . . . . . 193

5.18 Schematic showing layer-by-layer coating. . . . . . . . . . 195

5.19 ICR for PEI/PAA coatings. . . . . . . . . . . . . . . . 196 
5.20 Example of large current oscillations in TRPS following a 0 to $1.6 \mathrm{~V}$ switch. . . . . . . . . . . . . . . . . 199

5.21 Current oscillations with voltage. . . . . . . . . . . . . . 201

6.1 Schematic of concentration polarization. . . . . . . . . . 208

6.2 Pulse dependence on particle direction. . . . . . . . . . . . 212

$6.3 \Delta I_{c}$ with applied negative pressure. . . . . . . . 216

$6.4 \Delta I_{c}$ dependence on $\sigma \ldots \ldots \ldots \ldots$

$6.5 \Delta I_{c}$ dependence on electrolyte concentration. . . . . . . . . 222

$6.6 \Delta I_{r}$ and $\Delta I_{c}$ with $V_{0} \ldots \ldots \ldots \ldots \ldots \ldots \ldots \ldots \ldots \ldots \ldots \ldots$

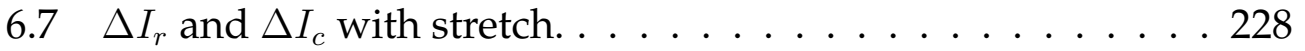

7.1 Typical size distribution measurements. . . . . . . . . . 236

7.2 Geometry used for modelling. . . . . . . . . . . . . . . 239

7.3 FEM resistive pulse simulations. . . . . . . . . . . . . 241

7.4 TRPS pulses from pressure reversal. . . . . . . . . . . 243 
7.5 Pulse size distributions as a function of stretch. . . . . . . . 247

7.6 CVs and peak distance with stretch. . . . . . . . . . 249

7.7 Size distributions with $P . \ldots \ldots . \ldots \ldots$

7.8 CVs, peak distance and peak height with $P \ldots \ldots 254$

7.9 CVs with varied stretch and $P \ldots \ldots \ldots$

7.10 Size distributions with $V$ and orientation. . . . . . . . . . 257

7.11 Pulse electrokinetic effects. . . . . . . . . . . . . . 259

B.1 SEM images of A20407 . . . . . . . . . . . . . 313

B.2 SEM images of A21820 . . . . . . . . . . . . . . 314

B.3 SEM images of A28818 . . . . . . . . . . . . . . . 314

B.4 SEM images of A32029 . . . . . . . . . . . . . . . 315

B.5 SEM images of A12353 . . . . . . . . . . . . . . . . . 315

B.6 SEM images of A23300 . . . . . . . . . . . . . 316

B.7 SEM images of A40357 . . . . . . . . . . . . . 316 
B.8 SEM image of A40338 . . . . . . . . . . . . . . 317

B.9 SEM images of A41972 . . . . . . . . . . . . . . . . 317

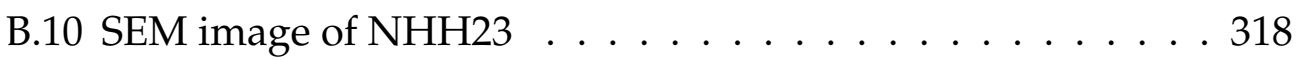

B.11 SEM image of A21032 . . . . . . . . . . . . . . 318

B.12 SEM image of A23802 . . . . . . . . . . . . . . . . 319

B.13 SEM image of A25135 . . . . . . . . . . . . . . . 319

B.14 SEM images of A27082 . . . . . . . . . . . . . . . 320

B.15 SEM images of A25136 . . . . . . . . . . . . . 320

B.16 SEM images of A33701 . . . . . . . . . . . . . . 321

C.1 $T_{n}$ for calibration particles . . . . . . . . . . . 324

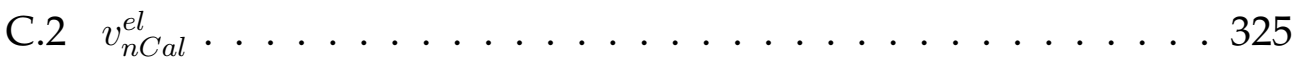

C.3 Averaged $v_{n C a l}^{e l} \ldots \ldots \ldots \ldots \ldots \ldots \ldots \ldots$

C.4 $T_{n}$ for sample particles $\ldots \ldots \ldots$. . . . . . . . 327

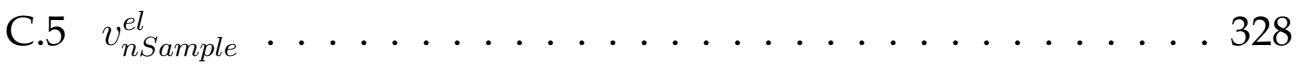




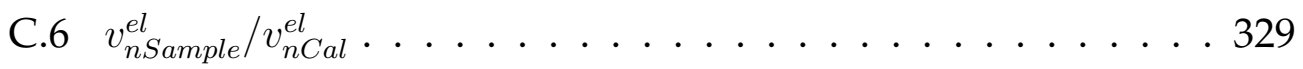

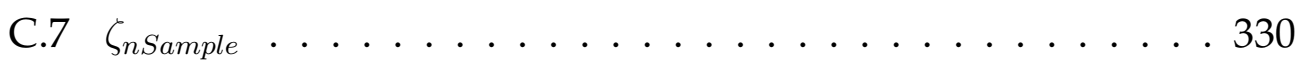




\section{List of Tables}

2.1 Izon Science nanopore specifications. . . . . . . . . . . . . 19

2.3 Summary of TRPS aggregation studies. . . . . . . . . . . 45

3.2 Summary of pores used. . . . . . . . . . . . . . 89

3.3 Summary of particles used. . . . . . . . . . . . . . 91

4.1 Summary of uncertainties. . . . . . . . . . . . . . . 125

4.2 Random error in $\zeta_{n \text { Sample }}-$ Method II. . . . . . . . . . . . . . 133

4.3 Systematic random error Method II. . . . . . . . . . . . . . . 133

4.4 Calibration particle $\zeta$-potentials. . . . . . . . . . . . . 139 
4.5 Random error in $\zeta_{n \text { Sample }}$ - Method IV. . . . . . . . . . . . 145

5.1 Random error in $\zeta_{n \text { Sample }}$ - Method IV. . . . . . . . . . . 170

5.2 FTIR assignments for Figure 5.9 . . . . . . . . . . 180

5.3 XPS survey assignments. . . . . . . . . . . . . . 182

5.4 High resolution XPS assignments. . . . . . . . . . . 183

$5.5 \zeta_{\text {pore }}$ and $R_{f}$ for PEI/PAA coating. . . . . . . . . . . 197

6.1 Summary of experimental conditions Ch. 6. . . . . . . . . 210

6.2 Particle sizing using biphasic pulses. . . . . . . . . . . . 230

7.1 Particle sets used in Ch. 7. . . . . . . . . . . . . . 246 


\section{Chapter 1}

\section{Introduction}

\subsection{Particle Characterization}

Nano and micro-particles are of great interest to researchers in a number of fields including medicine [1-3], biology [2, 4], electronics [5], materials science [6] and food technology [7]. Application and development of such particles in these areas requires an efficient and trustworthy method of detection and characterization. With a more complete understanding of these particles some important scientific advances can be made, such as specific, targeted drug delivery for cancer treatment with reduced side 
effects $[3,8]$ and on-bead sensors with a high degree of specificity for a range of chemical and biological targets that can be used in a lab on a chip scenario [9]. The two most common methods for particle analysis are dynamic light scattering (DLS) and electron microscopy (EM).

DLS can be used to elicit particle size and $\zeta$-potential from colloidal particles with diameters $1 \mathrm{~nm}$ up to $10 \mu \mathrm{m}$. The size of a particle suspension is measured by tracking the time-dependent intensity fluctuations of scattered light as particles undergo Brownian motion. $\zeta$-potential is calculated from electrophoretic mobility which is measured using laser doppler velocimetry. Particle speed in an electric field is monitored using light intensity fluctuations in a laser beam [10]. In DLS particle size measurements there is a bias towards larger particles, because light scattering intensity is proportional to particle diameter to the power of 6 , so that even a few large particles in solution can dominate the signal [11]. Combined with the ensemble (i.e. population averaging) nature of the technique, this limits the usefulness of DLS for polydisperse samples or any samples that aren't completely homogeneous [12].

There are two main types of EM, transmission electron microscopy (TEM) and scanning electron microscopy (SEM). In both cases, electron 
beams are used to image a sample. These images can be used to produce number-based size distributions. Because particles are being imaged, detailed shape information is also available, which is not the case in the majority of comparative techniques. However, the technique is labour intensive as it takes comparatively more time and effort to achieve similar particle counts to other techniques. Some EM also requires a vacuum which removes the possibility of in solution particle analysis [12]. However, recent developments such as environmental SEM enable viewing of particles in solution [13].

Resistive pulse sensing (RPS) is an alternative particle analysis technique. A typical resistive pulse sensor consists of two fluid reservoirs, each filled with aqueous electrolyte, partitioned by a membrane containing a connecting aperture (Figure 1.1). When a voltage $\left(V_{0}\right)$ is applied to electrodes on either side of the membrane, a current $\left(I_{0}\right)$ is carried through the aperture by mobile ions in the electrolyte. Particles are conventionally detected using the transient increase in resistance, or resistive pulse which is also referred to as a blockade event, generated as they pass through the aperture. The magnitude, frequency and duration of these resistive pulses are dependent on the size, concentration, and charge of the particles, as 


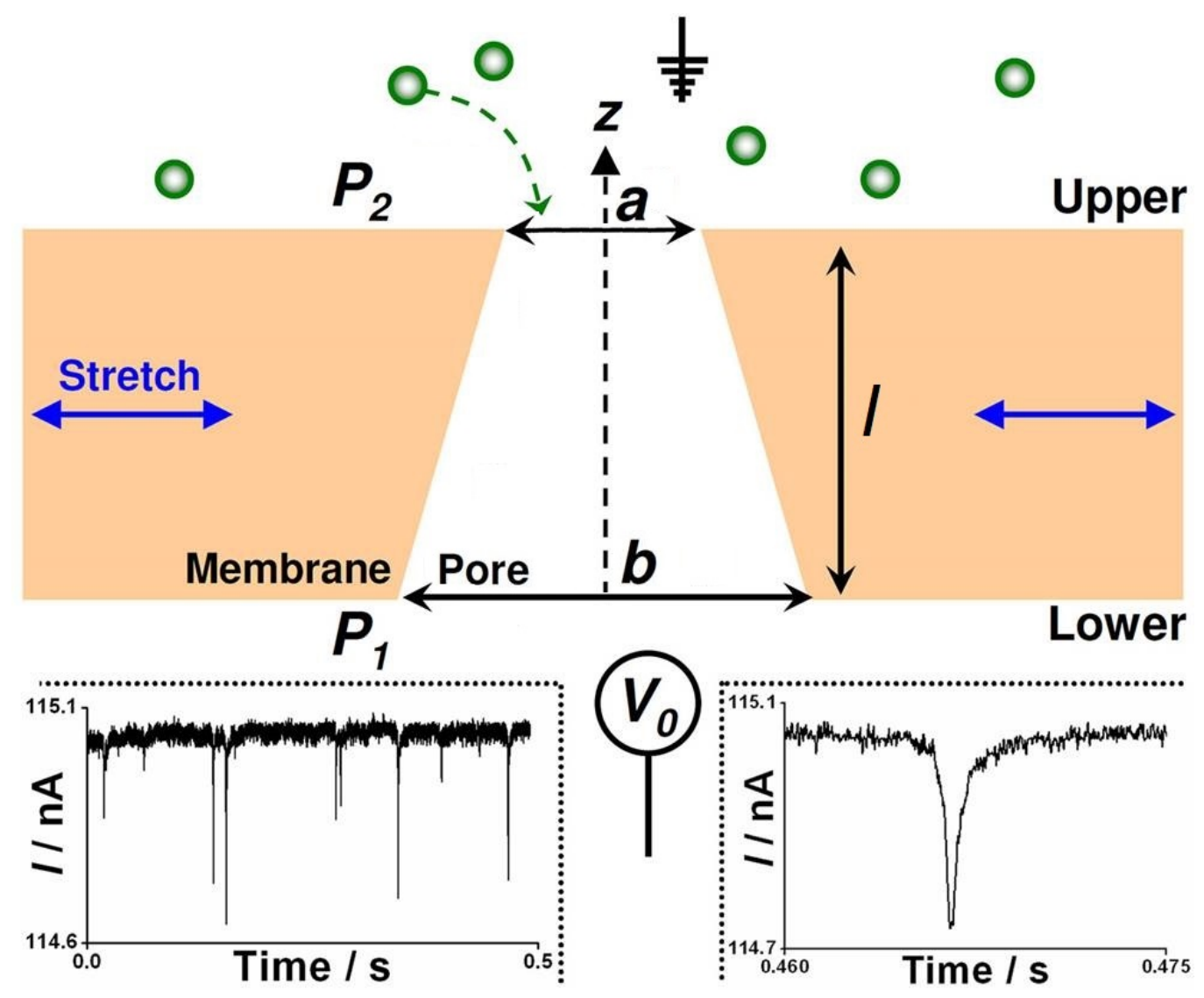

Figure 1.1: Schematic section through a tunable pore, indicating experimental variables usually used in tunable resistive pulse sensing (TRPS), an interesting type of resistive pulse sensing (RPS). The conical pore of length $l$ has opening diameters $a$ and $b$, and is assumed to be symmetric about the cylindrical $z$-axis. Potential $V_{0}$ and pressure $P_{2}-P_{1}$ are applied across the membrane. Lower left, typical experimental data showing resistive pulses. Lower right, one pulse in greater detail. Adapted from Somerville et al. [14] 
well as the aperture geometry. Hence, measurements can be used to calculate these characteristics.

\subsection{Early Resistive Pulse Sensing}

The RPS particle analysis technique, also known as the Coulter principle, was developed and patented in the 1950s by Wallace H. Coulter, an American electrical engineer, with the aim of automating the routine red blood cell (RBC) count [15]. Up until the 1950s blood analysis was achieved by laboriously counting cells in a standard haemocytometer counting chamber under a microscope. The introduction of Coulter Counters substantially improved the throughput and accuracy of complete blood counts, increasing the number of cells counted per sample by approximately 100 times by counting in excess of 6000 cells per second, reducing statistical counting errors by approximately 10 times and giving an 120-fold reduction in measurement time [16]. Nowadays the complete blood count is the most commonly ordered medical test worldwide and instruments such as haematology analysers and flow cytometers employed by haematology laboratories use the Coulter principle for cell analysis [16]. 
Coulter Counters have a variety of aperture sizes available with diameters between $20 \mu \mathrm{m}-2 \mathrm{~mm}$ in diameter. Apertures are bored through a man-made sapphire membrane of a comparable thickness to their diameter and embedded in a glass tube. A typical, modern day, Coulter Counter is capable of detecting particles larger than $500 \mathrm{~nm}$ in diameter, including microorganisms, blood cells, spermatozoa, platelets, algae and yeasts as they are forced through the aperture under pressure-driven flow [17].

RPS was first applied to sub-micron analytes in the 1970s by DeBlois and Bean who examined $60 \mathrm{~nm}$ diameter polystyrene spheres, as well as $\mathrm{T} 2$ and $\mathrm{T} 4$ bacteriophages (approximately $90 \mathrm{~nm}$ in diameter), and a range of insect and animal virus particles [18-21] using track-etched polycarbonate membranes. An early comparative study showed that RPS measurements were in agreement with established techniques such as DLS, EM and spectrometry. It was noted that RPS and EM thrived for polydisperse samples where DLS lacked resolution [21]. Following DeBlois and Bean's work further advances in RPS did not transpire until the mid-1990s.

The resurgence of RPS was spearheaded by Kasianowicz et al. in a 1996 study demonstrating the use of a $2.6 \mathrm{~nm}$ Staphylococcus aureus (S. aureus $\alpha$-hemolysin ( $\alpha$-HL) protein pore embedded in a lipid bilayer for 


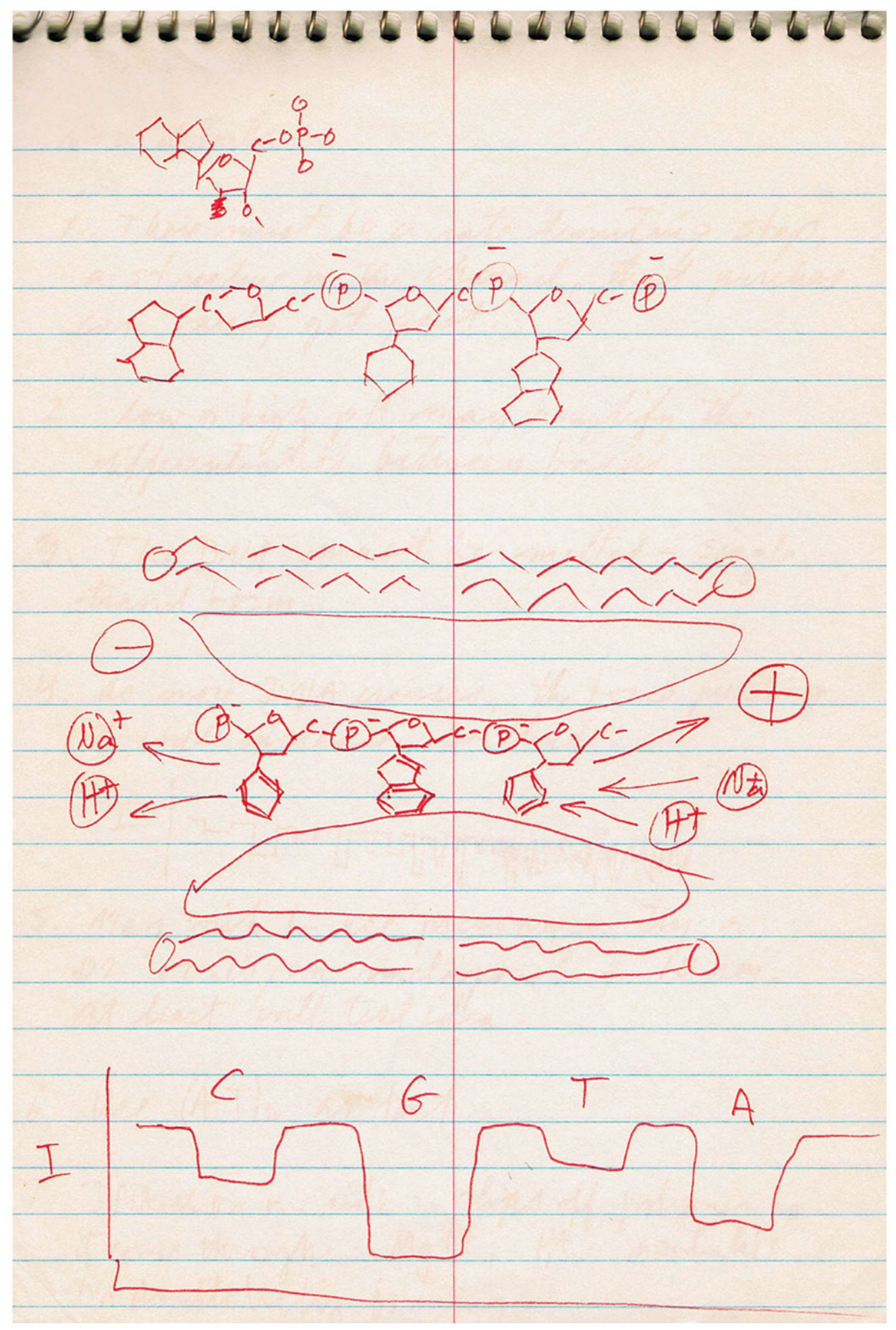

Figure 1.2: Sketch of the original nanopore sequencing concept from the notebook of Deamer who worked alongside Kasianowicz to measure ssDNA using $\alpha$-HL pores. The sketch was dated 1989. Adapted from Deamer et al. [22]. 
single strand (ss) DNA detection (Figure 1.2) [23]. Subsequently, it has been shown that $\alpha$-HL can be used to differentiate between individual Watson-Crick base pairs at the ends of DNA hairpin molecules [24]. The suggestion that a refinement of this technique may allow for sequencing of long native DNA molecules at high speed has generated considerable research interest in the use of nanopores for DNA sequencing and the idea of Coulter Counter miniaturization [25]. This renewed interest in combination with the advances in synthetic biology and nanofabrication techniques has given rise to a myriad of synthetic and biological pore sensors which are capable of counting and sizing a range of particle types from microparticles to single molecules.

Currently, the field of nanopore sequencing is led by Oxford Nanopore Technologies Limited, a UK-based nanopore sequencing company [26]. Founded in 2005, the company released their portable protein nanopore sequencing USB device "MinION" to around 1000 select early access researchers, including NASA for use at the international space station, in May 2015. Inital reports have been mixed, some suggesting that "MinION" results are too erroneous to compete with existing sequencing technologies [27, 28]. Recent reviews have covered analysis of nucleic acids us- 
ing biological nanopores [29-31] and the broader field of molecular-scale RPS [25, 32-40].

\subsection{Introduction to Tunable Resistive Pulse Sens- ing}

Tunable resistive pulse sensing (TRPS) [41-52], a variant of RPS, is distinctive because the membrane, containing the pore, is an elastomer. When the membrane is stretched on macroscopic length scales, the nanoscale dimensions of the sensing pore are mechanically 'tuned'. The most immediate advantages of tuning are pragmatic, such as recovery from a blockage when the membrane is stretched. Pores can be adjusted to suit a sample, so a complete understanding of a sample is not required before measurement. This also means that pores can be used multiple times over a range of different samples making them more economical than single-use pores. The signal-to-noise ratio of the resistive pulse signal can be optimised in situ, during an experiment, to optimise the signal. This allows for detection and discrimination of individual particle populations in a polydisperse sample [53]. TRPS therefore has a flexibility unavailable with static 
pores used for RPS.

First reported almost a decade ago [41], TRPS reached essentially its present form in terms of technical development by 2010 [42] and there are now established TRPS protocols for measurement of particle size [43], concentration [42, 44], and charge [46-48]. Izon Science Ltd., a New Zealand technology company [54], have designed and manufactured a particle analysis platform, the qNano, which utilises these pores and the concept of TRPS. Izon Science have sold over 200 instruments which are in use in a wide range of research institutes and universities around the world [54]. Much of this development work has used spherical polystyrene colloids, commercially available as size standards. The range of particle sizes measurable using TRPS spans from micrometers down to $\sim 50 \mathrm{~nm}$, covering approximately two orders of magnitude in length scale and bridging the important gap between single molecules and cells [49]. A variety of electrolytes can be used, including those which replicate physiological conditions, and $100 \mathrm{mM}$ salt is typical. The accessible range of particle concentrations is approximately $10^{5}-10^{12} \mathrm{~mL}^{-1}[55]$.

Further advancement of this technology requires a better understanding of the technique. In particular, a more rigorous study of how important 
experimental variables affect the accuracy and precision of TRPS particle characterization measurements, especially particle charge, is crucial. An investigation of particle charge using the qNano system will also test fundamental understanding of colloidal particle charge. Experimental investigation of often overlooked phenomena, such as conductive and biphasic pulses at low electrolyte concentrations and broadening or artefacts in particle size distributions, increases fundamental scientific understanding and is also important for assessing the accuracy of particle characterisation using this technique.

This Thesis will study nanoparticle detection in response to evolving scientific directions and applications. To the existing body of work this Thesis adds an exhaustive review of the applications of TRPS, a thorough investigation of surface charge measurements using TRPS including the development of a pore charge measurement method, the discovery and in-depth examination of conductive and biphasic pulses in TRPS, and the rigorous investigation of particle size distribution broadening and double peaks for monodisperse particle samples. 


\subsection{Thesis Structure}

This Thesis consists of 8 chapters. Chapter 2 is an extensive literature review of TRPS providing a guide to the characteristics of TRPS with emphasis on the roles it has played in applied research. Chapter 3 presents the materials and methodologies used in experimental Chapters 4, 5, 6 and 7.

Chapters 4 and 5 describe $\zeta$-potential measurements made using TRPS with an initial and then refined measurement methodology respectively. In Chapter 6 describes the investigation of conductive and biphasic pulses and how particle charge and other experimental variables affect these pulses and the information that can be obtained from them. Chapter 7 investigates the similarly often bypassed phenomenon of peak broadening and double peaks in monodisperse particle samples. The final Chapter provides a summary and concluding remarks as well as highlighting possibilities for future TRPS research. 


\section{Chapter 2}

\section{Literature Review}

TRPS is an experimental technique capable of particle-by-particle detection and analysis of submicron colloids and bioparticles [41-52]. The purpose of this Chapter is to provide a guide to the characteristics of TRPS, with emphasis on the roles it has played in recent applied research. The Chapter covers TRPS research into a wide range of particle types, encompassing numerous research fields and research objectives. It draws on information sources that will be of specific relevance to particular TRPS investigations, and also those that promote interdisciplinary understanding

of the technique. The Chapter demonstrates the challenges of colloidal 
characterization, such as the often complex nature of raw samples (e.g. bodily fluids), the importance of understanding the distinct principles behind different measurement techniques, and the sheer variety of particle types studied.

This Chapter does not detail RPS work at comparable length scales using static, solid-state pores. Such studies have made use of pore materials including silicon-based membranes [56, 57], carbon nanotubes [58, 59], and lithographically moulded [60] and track-etched [33, 61, 62] polymers. Glass pores have been made by laser machining [63-66], the pipette pulling technique $[67,68]$, and moulding around a sharp wire tip [69-72]. Henriquez et al. [73] and more recently Luo et al. [74] have reviewed aspects of static RPS experiments. TRPS has a particular similarity to RPS studies in which the pore geometry is conical $[33,61,66-68,75,76]$.

The first Section ("Technical Aspects") introduces the technology by recounting descriptions of TRPS and sensing characteristics, including particle charge which will be an important theme throughout this Thesis, and then identifying areas of ongoing technical development. Comparisons with other methods are reviewed, revealing some advantages and distinct characteristics of TRPS. The second Section ("Applications") re- 
views application of TRPS to a broad range of particle types. This Section summarizes important results and serves as a guide either to the studies most relevant to each specific experimental area, or to new perspectives from different fields of research. Studies are broadly classified by application area: diagnostics and genomics, extracellular vesicles (EVs), nanomedicine, phages, viruses and bacteria, and others. Inevitably, the boundaries between classifications can be indistinct, as in the case of structurallysimilar EVs, liposomes and emulsions.

Much of the material in this Chapter has been published in [77]. Willmott provided discussion and suggestions on the writing style and content of the paper.

\subsection{Technical Aspects}

\subsubsection{Pore Specimens and Stretching}

Tunable pore specimens (Figure 2.1), commercially available from Izon Science (Christchurch, NZ) [54], are formed by controlled puncture of a thermoplastic polyurethane (TPU) membrane using a chemically etched 

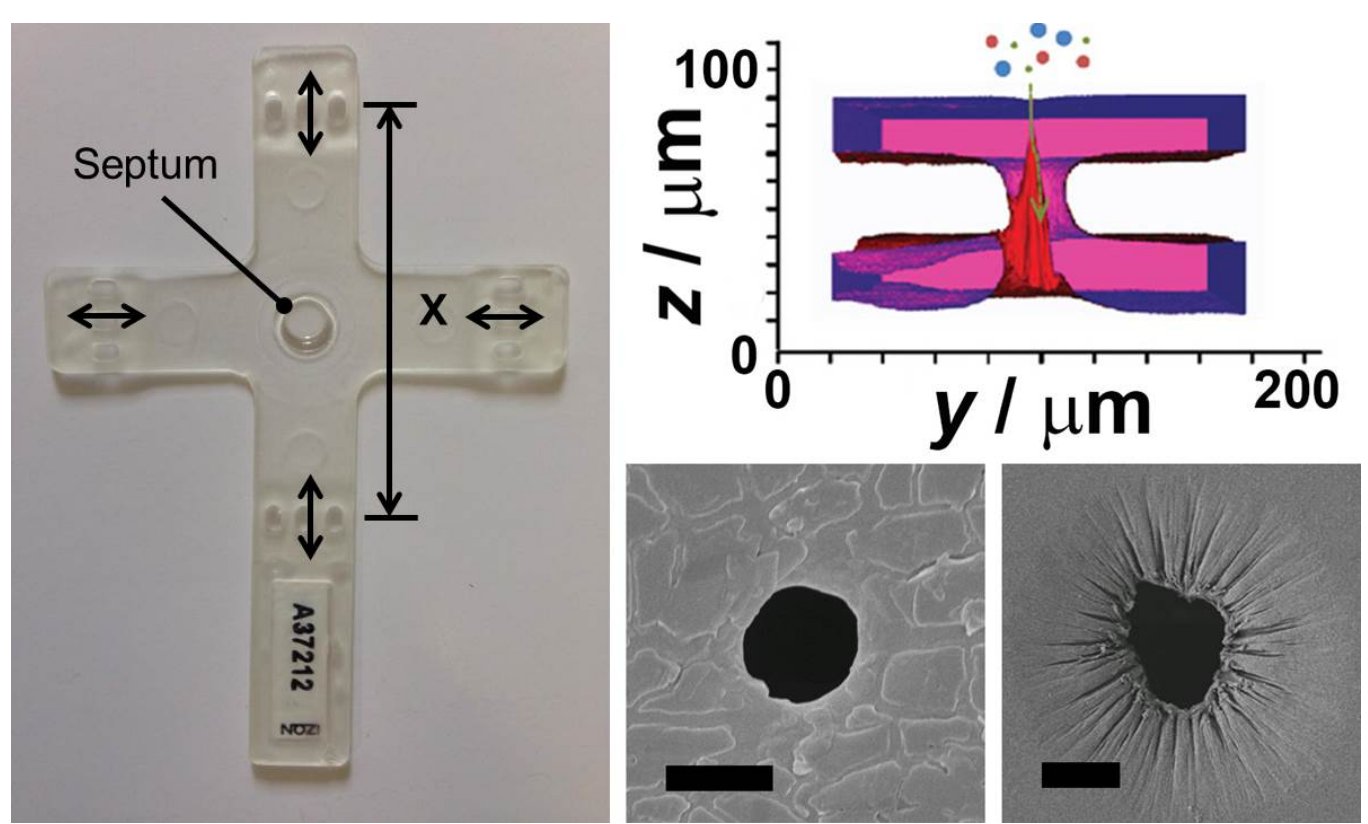

Figure 2.1: Specimen imaging. Left, a TPU specimen. $X$ is $\sim 42 \mathrm{~mm}$ for an unstretched specimen, and the pore is located near the centre of the $\sim 200$ $\mu \mathrm{m}$ thick circular septum. Reproduced from Willmott et al. [78]. Right upper, reconstruction of a pore cross-section from confocal microscopy, adapted from Kozak et al. [49]. Right lower, SEM images of smaller (left) and larger (right) openings of a pore stretched to $X=45 \mathrm{~mm}$ (scale bars 1 $\mu \mathrm{m}$ and $20 \mu \mathrm{m}$ respectively), adapted from Willmott et al. [78]. 
tungsten needle [41]. TPU is a randomly segmented co-polymer composed of hard, crystalline segments containing hydrogen bonded amine and carboxyl moieties which cluster together, and soft amphorous dopamins. The length of these domains can vary from 10s to 100s of nanometers. TPU is stable and inexpensive, and can be extended to high strains without causing large plastic deformations or breakage [51]. The pore making process is relatively efficient in comparison with the intensive techniques necessary to make pores for detecting single molecules [25] - such as electron or ion beam lithography, or use of biological pores. An approximately conical hole is produced, with larger and smaller pore openings (Figures 1.1 and 2.1). Depending on the needle [48] and fabrication parameters, commercially-available pores are given a rating indicating their relative size, and therefore the particle size they are most suited to sensing (Table 2.1). SEM $[14,42,43,47,50-52,79]$ has enabled imaging of a variety of pores at different stretches, and measurement of pore opening sizes, while confocal microscopy can provide three-dimensional information $[42,49$, 53] (Figure 2.1) and atomic force microscopy has also been used for imaging [52].

The membrane is the $\sim 200 \mu \mathrm{m}$ thick central septum of an injection- 
moulded sample (Figure 2.1). Each cross-shaped sample is stretched biaxially and symmetrically by adjusting the separation of teeth placed in the eyelets on the ends of the arms $[42,78]$. Stretching can be quantified using the distance $X$ between the teeth on opposite arms of the specimen, and $X_{0}$, which is the value of $X$ when no stress is applied ( $\sim 42 \mathrm{~mm}$ at manufacture) $[42,52,78,80]$. TPU (as with all elastomers) is viscoelastic, but mechanical reproducibility can be maximised by stress-cycling $[51,78,81]$. Macroscopic membrane actuation changes the micro- or nanoscale pore geometry and ionic resistance, and these relationships have been modelled both empirically $[51,52,78,82]$ and using finite element approaches $[78,80]$. Models for stretching $[78,80,82]$ have been used to calculate membrane thickness in experiments $[14,42,48,83]$. Membrane thickness can be measured using confocal images $[43,79]$ or a modified micrometre screw gauge $[47,50,82,84]$.

\subsubsection{Apparatus}

The commercial qNano instrument (Izon Science) [42] builds on earlier TRPS apparatus $[41,51,52]$. The qNano (Figure 2.2) is a cylindrical chamber $\sim 30 \mathrm{~cm}$ tall and $\sim 12 \mathrm{~cm}$ in diameter. It holds a pore specimen in a 
Table 2.1: Manufacturer's nanopore specifications. Sample particle analysis range and recommended particle concentration for optimal results are given. Recommended carboxylated polystryene calibration (CPC) particles for each pore are listed in the far right column [54]. The nominal diameter of particles is listed after CPC e.g. CPC100 are nominally 100 nm.

\begin{tabular}{|c|c|c|c|}
\hline Pore Size & Target Particle Analysis Range $(\mathrm{nm})$ & Concentration $(/ \mathrm{mL})$ & Calibration Particles \\
\hline NP100 & $40-320$ & $1 \times 10^{10}$ & CPC100 \\
\hline NP150 & $60-480$ & $5 \times 10^{9}$ & CPC100, 200 \\
\hline NP200 & $80-640$ & $2 \times 10^{9}$ & CPC200 \\
\hline NP300 & $115-1150$ & $1 \times 10^{9}$ & CPC200, 400 \\
\hline NP400 & $155-1550$ & $5 \times 10^{8}$ & CPC400, 500 \\
\hline NP800 & $320-3200$ & $1 \times 10^{8}$ & CPC500, 800 \\
\hline NP1000 & $400-4000$ & $5 \times 10^{7}$ & CPC800, 1000 \\
\hline NP2000 & $800-8000$ & $5 \times 10^{6}$ & CPC2000 \\
\hline NP4000 & $1600-16000$ & $1 \times 10^{5}$ & CPC4000 \\
\hline
\end{tabular}

fluid cell at the top of the device. The complete fluid cell is comprised of a lower fluid cell, upper fluid cell and shielding cap (Figure 2.2). The lower fluid cell contains a fluid well and one of the $\mathrm{Ag} / \mathrm{AgCl}$ electrodes. It is connected to the instrument's electronics via a SubMiniature version A (SMA) connector. The specimen is placed on top of the lower fluid cell with its eyelets fitted over the stretching jaws. The upper fluid cell slots into the lower fluid cell, resting on top of the pore. It contains a central, bottomless fluid well which sits atop the pore septum, as well as the second $\mathrm{Ag} / \mathrm{AgCl}$ electrode. The shielding cap is placed on top of the fluid cell and essentially acts as a Faraday cage, minimising electrical interference.

The stretching jaws sit either side of the fluid cell. As described above 


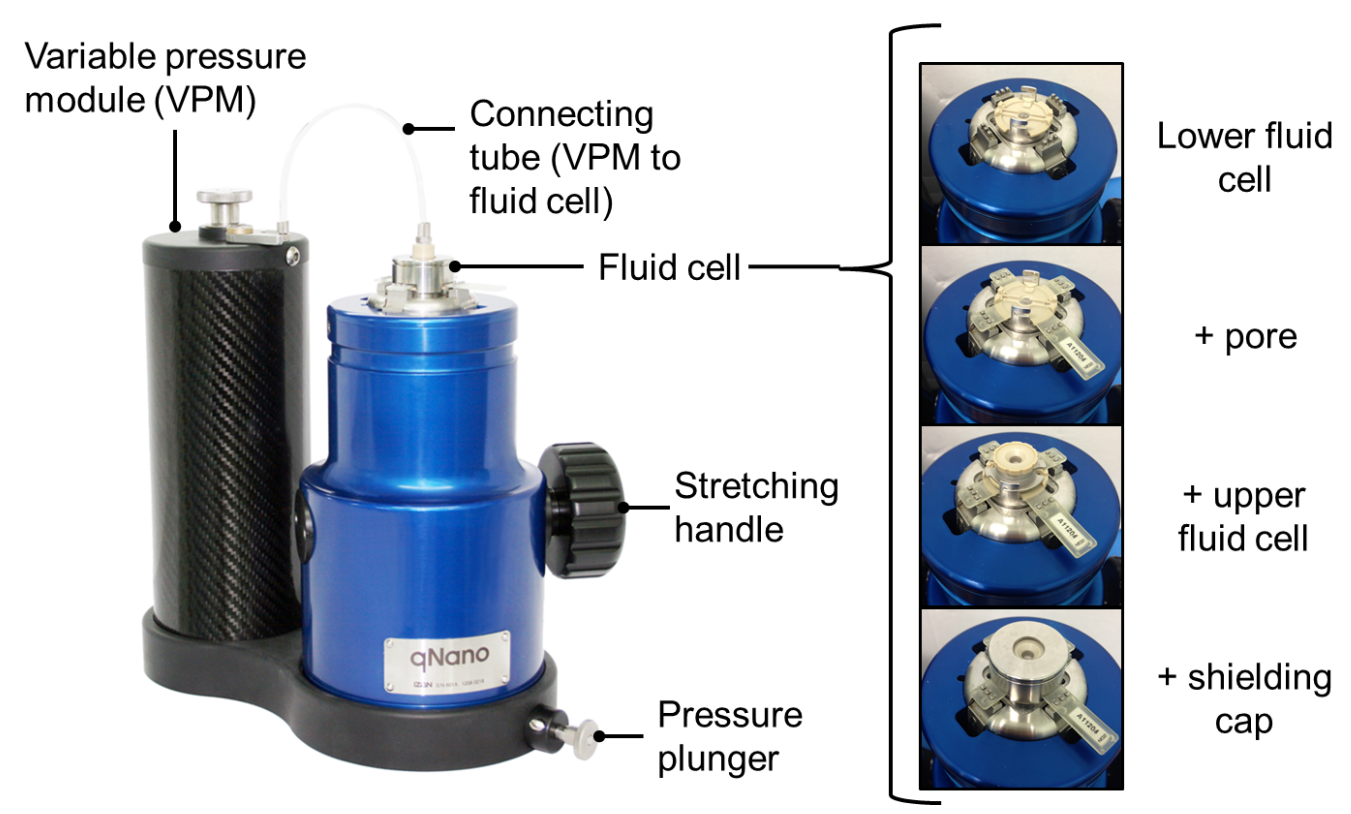

Figure 2.2: The qNano (left, Izon Science) TRPS apparatus, and (right) the magnified fluid cell. Ag/AgCl electrodes contact each half of the cell. Actuation is achieved by turning the handle on the side of the qNano, which moves teeth placed in the specimen. The black cylindrical variable pressure manometer (VPM) connects to the top of the fluid cell via tubing. Adapted from Willmott et al. [78] and Weatherall et al. [48]. 
the stretch $X$ applied to the pore specimen is measured between sets of teeth placed in holes at the ends of opposite arms on the specimen, and is symmetric across the two pairs of arms. The qNano is positioned on a platform attached to the variable pressure manometer (VPM), which connects to the instrument via tubing to the upper fluid cell. Precise application of pressure across the membrane controls pressure-driven liquid flows through a pore, and this is enabled by the gravitational pressure head of water within a water VPM [47], or the air pressure using an air VPM, along with knowledge of the inherent pressure head in the fluid cell $[43,46]$. Signal noise and bandwidth are important [63], and undersampling occurs if particles are driven too quickly through a pore, causing the pulse duration to approach the $50 \mathrm{kHz}$ sampling rate [74].

Other Izon Science technologies include the qViro and the qMicro. The qViro (also known as the qViro-X) apparatus uses the same specimen type, and can withstand the decontamination processes required for experimentation with viruses. The qMicro again uses the same specimen type, but is designed for micron sized particles in the $4-300 \mu \mathrm{m}$ range. For microparticle analysis the whole sample is run through the pore and size and concentration information is extracted. Further bespoke developments 
(e.g. high frequency actuation [81] and co-ordination with optical techniques $[85,86])$ can be anticipated.

\subsubsection{Analysis and Measurement Protocols}

Simple, efficient resistive pulse analysis is necessary for high-throughput experimentation. Introduced here is the physical modelling that has been used to develop the existing TRPS protocols for measuring particle concentration, size, and charge of particles in general particle characterization experiments. In the regime relevant to TRPS, models are typically constructed by calculating resistive pulse magnitude as a function of particle position, and separately calculating the motion of a particle by considering transport mechanisms acting upon it. Separation of these calculations is justified by the relatively small timescale of ionic diffusion [87].

A semi-analytic technique has been used to calculate resistive pulse size as a function of particle position in TRPS [88], based on previous RPS work $[49,51]$. Early analyses for cylindrical $[19,20]$ and conical pores [61] suggest that the pore's electrical resistance to ionic current $R_{0}$ can take the form 


$$
R_{0}=\int \frac{\rho}{A(z)} d z
$$

where $A(z)$ is the pore's cross sectional area at position $z$ (Figure 1.1). Here it is assumed that the electrolyte resistivity $\rho$ is homogeneous, because the size of the electrical double layer (EDL, e.g. ranging between 10 to $1 \mathrm{~nm}$ for 1 to $100 \mathrm{mM} \mathrm{KCl}$ ) is small relative to usual TRPS geometry. The access resistance beyond the ends of the pore [89] $\left(R_{e n d}=\frac{\rho}{4 a}\right.$ for opening radius $\left.a\right)$ is usually included [42]. If a particle (an insulating sphere) is introduced, the new pore resistance can be numerically calculated using the same approach. By implementing this technique, models $[79,88]$ have reproduced experimental resistive pulse asymmetry, enabled resistance calculations when a particle is partly or fully outside of the pore, and found that pulse size should be maximised when the particle is not entirely within a conical pore $[53,89]$.

The particle position as a function of time can be calculated using the Nernst-Planck approach [90]. Transport mechanisms give a vector sum for the particle flux $\mathbf{J}$ (units: particles $\mathrm{m}^{-2} \mathrm{~s}^{-1}$ ), taking the form 


$$
\begin{aligned}
\mathbf{J} & =\left(\mathbf{J}_{e p}+\mathbf{J}_{e o}\right)+\mathbf{J}_{p d f}+\ldots \\
& =\frac{C \varepsilon}{\eta}\left(\zeta_{\text {particle }}+\zeta_{\text {pore }}\right) \mathbf{E}+\frac{C \mathbf{Q}}{A}+\ldots
\end{aligned}
$$

The specified terms represent fluxes driven by the three most important mechanisms for TRPS: electrophoresis $\left(\mathbf{J}_{e p}\right)$, electroosmosis $\left(\mathbf{J}_{e o}\right)$ and pressure-driven flow $\left(\mathbf{J}_{p d f}\right) . C, \varepsilon$ and $\eta$ are respectively particle concentration, solution permittivity and viscosity. $\zeta$ is the $\zeta$-potential of the subscripted surface, $\mathbf{E}$ is the electric field and $\mathbf{Q}$ is the volume flow rate. The explicit expressions for electrophoresis and electroosmosis are appropriate for a thin EDL, and all three terms require that the pore wall has a low gradient, approximating a cylinder $[47,90]$. Development of transport modelling for TRPS was recently summarized, with the relative importance of transport mechanisms compared over a range of geometries [91].

Together, Equations 2.1 and 2.3 can be used to construct resistive pulses $[46,79,83]$. The model developed for TRPS has been used elsewhere [92], and there are relevant RPS studies of transport mechanisms [56, 69, 70, 93]. Previous finite element modelling (FEM) of TRPS [88, 94] has used 
comparable methodologies to conical pore RPS simulations $[68,76,87,95]$, and has been compared with the semi-analytic model [88].

\section{Concentration Measurements}

When transport is dominated by pressure-driven flow, the pulse rate is proportional to the flow rate and independent of chemical and physical differences between particles (Equation 2.2). The Nernst-Plank equation can be shortened to

$$
\mathbf{J}_{p d f}=\frac{C \mathbf{Q}}{A} .
$$

Modification of the Hagen-Poiseuille equation for flow in a tube to account for the conical geometry of the pore results in the following expression for Q [88]:

$$
\mathbf{Q}=\frac{3 \pi P}{8 \eta l} \frac{b-a}{b^{3}-a^{3}} a^{3} b^{3}
$$

Here $P$ is the applied pressure, $l$ is the length of the pore, $a$ is the radius of 
the small pore opening and $b$ is the radius of the large pore opening.

Using standard polystyrene particles, the pulse rate has been demonstrated to vary linearly with pressure up to $\sim 1.8 \mathrm{kPa}[42,45]$ and with concentration between approximately $1 \times 10^{8}$ and $5 \times 10^{10} \mathrm{~mL}^{-1}[42,47,53]$. Independence from particle type has been confirmed by verifying these relationships with particles such as virions and bacteria $[47,53,96]$, and liposomes [97].

Concentration of an unknown particle set can be calculated by calibrating the pressure-driven flow using particles of known concentration. The most commonly used calibration method uses the linear relationship between the particle rate $J \times A$ and $P$. As per Equations 2.4 and 2.5 the gradients of rate against pressure can be compared for particles of known and unknown concentration in order to extract the unknown concentration

$$
C_{2}=\frac{g_{2}}{g_{1}} C_{1}
$$

where $C_{1}$ and $g_{1}$ are, respectively, the concentration of the calibration sample, and its associated gradient taken from a linear fit to the plot of mea- 
sured particle count rate against pressure, $g_{2}$ is the same gradient associated with the sample of unknown concentration, and $C_{2}$ is the unknown concentration which can then be calculated.

Alternative concentration methods have been explored, and include a calibration-free method using geometric parameters [47, 53] (Equations 2.4 and 2.5) and an internal calibration technique [98]. These avoid the requirement for separate sample and calibration measurements and could therefore be advantageous for complex biological media.

Roberts et al. investigated the accuracy of both calibration and calibrationfree methods [53]. The external calibration method was shown to be accurate to within 3\% when calibrating different dilutions of $400 \mathrm{~nm}$ carboxylated polystyrene particles to each other. It was noted however, that the validity of these results was dependent on the accuracy of previous concentration measurements made by the manufacturer. Following this the concentration of $1 \mu \mathrm{m}$ Baculovirus occlusion bodies and cyanobacterium Prochlorococcus were also measured using the external calibration method and were found to be within $17 \%$ of microscopy and within $6 \%$ of flow cytometry (FC) measurements respectively. The calibration-free method was shown to have a poor level of accuracy. An $86 \%$ difference was found be- 
tween the calculated concentration and the manufacturer's concentration, for a sample of $400 \mathrm{~nm}$ National Institute of Standards and Technology traceable polystyrene particles. This lower level of accuracy was attributed to the imperfect conical shape of the pore and possible changes in the pore geometry between the geometric measurements using microscopy and the TRPS experiments.

\section{Size Measurement}

The most well-established particle sizing method for RPS uses the principle that particle volume is proportional to the corresponding pulse magnitude. This principle was established using cylindrical pores [18, 19] and was first demonstrated with TRPS by Vogel et al. [43], who also showed that results were consistent with the semi-analytic model (based on Equation 2.1) for conical pores.

The resistance of an electrolyte filled pore is given by Equation 2.1 and can be written more specifically for a slowly-varying conical pore as [61]

$$
R_{0}=\frac{4 \rho l}{\pi a b}
$$


Including end effects this becomes [88]

$$
R_{0}=\frac{4 \rho[l+0.4(a+b)]}{\pi a b}
$$

Again, $\rho$ is homogeneous throughout the system. This is consistent with the typical Ohmic response of pores and the fact that TRPS experiments generally take place in the high-salt limit where the Debye length is much smaller than the pore dimensions [99].

As an insulating particle translocates the pore it increases the overall resistance $(\Delta R)$. The first model for calculating $\Delta R$ from particle size in a long cylindrical pore was developed by Maxwell [100] and later Lord Rayleigh [101]. This work showed that the cause of the increase in resistance was the displacement of a volume of electrolyte from the pore. This volume of electrolyte was equal to the volume of the particle and hence $\Delta R$ was proportional to the volume of the particle in the small-sphere limit ( $d<<D$, where $d$ is the particle diameter and $D$ is the pore diameter),

$$
\Delta R=\frac{4 \rho d^{3}}{\pi D^{4}}
$$


This expression was first applied to particles and compared to other sizing methods by DeBlois in the 1970s [18] who was able to extend this model to a wider range of particle sizes using the relation [19]

$$
\Delta R=\frac{4 \rho d^{3}}{\pi D^{4}}\left[1-0.8\left(\frac{d}{D}\right)^{3}\right]^{-1}
$$

For conical pores, $\Delta R$ can be determined by integrating the resistance between the end of a conical pore with and without the presence of a particle

$$
\Delta R=\rho \int \frac{d z}{A(z)}-R_{0}
$$

where $R_{0}$ is given by Equation 2.8. The key outcome of this equation is that $\Delta R$ is largely proportional to the volume of the particle, which is the basis for the most commonly used TRPS size measurement method demonstrated in experiments by Vogel et al. [43].

Calibration-free methods are possible. If the pore geometry is known the particle diameter can be calculated directly from the resistive pulse. However, irregularities in pore shape, changes in pore shape with stretch, and the time consuming and possibly destructive nature of pore geometry 
determination make calibration a much simpler and more commonly used method [43].

When comparing resistive blockade magnitudes $(\Delta I)$ for a calibration sample of known size and an unknown sample measured under identical conditions (same pore, stretch, pressure, voltage), the ratio of maximum blockade magnitudes is equal to the ratio of the volumes of the particles. Hence the diameter of the unknown sample can be calculated using

$$
d_{2}=\sqrt[3]{\frac{\Delta I_{2}}{\Delta I_{1}} d_{1}^{3}},
$$

where $d_{2}$ is the unknown particle diameter, $\Delta I_{2}$ is the maximum blockade magnitude of the unknown sample, $\Delta I_{1}$ is the maximum blockade magnitude of the calibration sample and $d_{1}$ is the calibration particle diameter. The calibration method is the approach recommended by Izon Science and implemented by their proprietary software [54].

The use of TRPS for particle sizing was first demonstrated by Vogel et al. [43] where the calibration method was used to measure the size of a range of biological and synthetic particles and gave results in agreement 


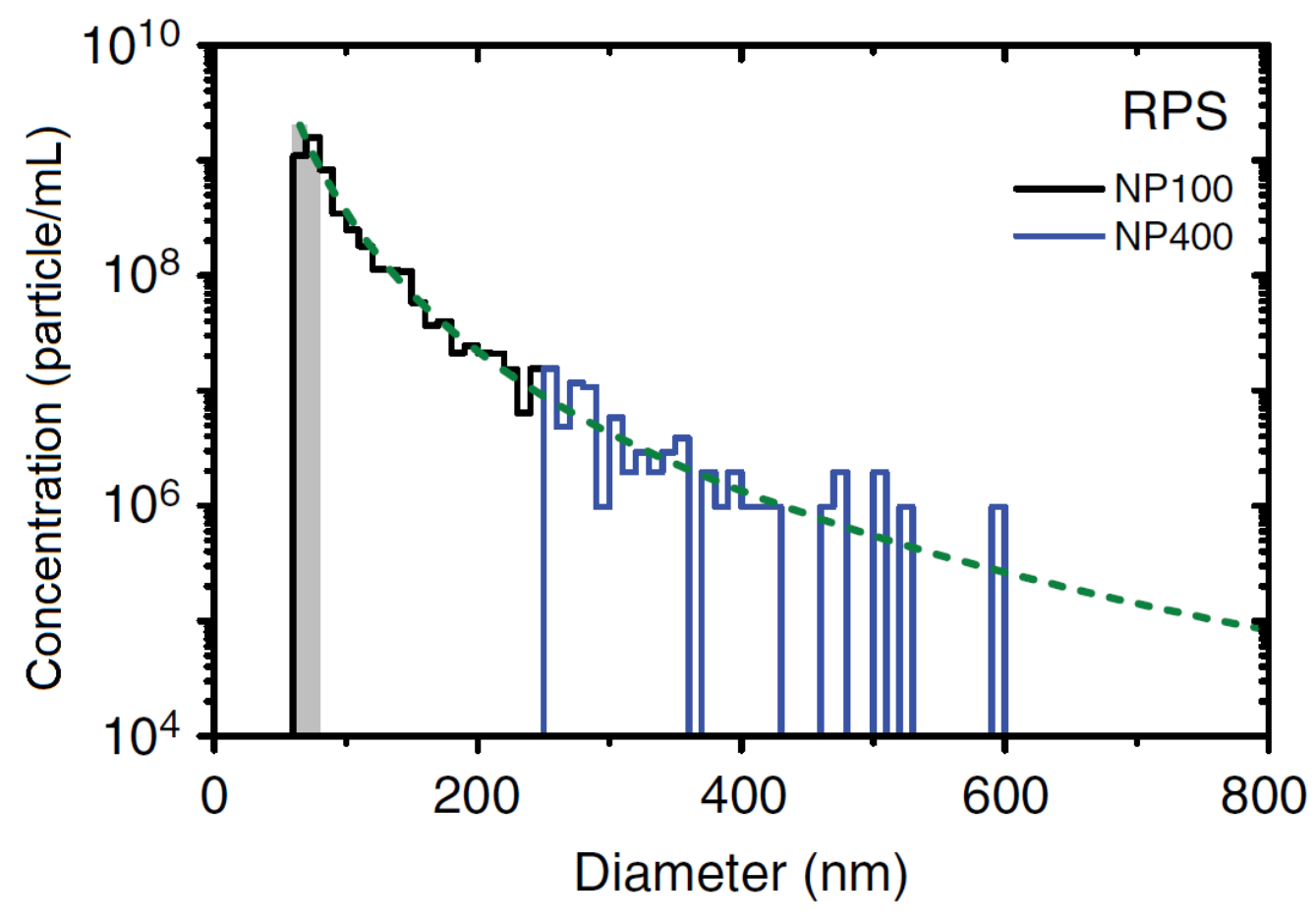

Figure 2.3: Size distributions for urinary EVs obtained using two tunable pores of different size (black and blue data) are combined to give a wideranging distribution, fitted by power law (green dashes). Reproduced from van der Pol et al.[102]. 
with TEM and DLS. Since then, TRPS size measurements have been carried out at a range of pore stretch settings [82], using multi-modal distributions $[12,53,98,103]$, and by combining size distributions obtained using pores of different sizes (Figure 2.3) [103]. Arjmandi et al. recently used TRPS apparatus to demonstrate a sedimentation technique for nanoparticle mass measurement [104], citing the need for new mass measurement methods for virions in particular.

Particles are usually assumed to be spherical for analysis, and in this Thesis sizes refer to the equivalent spherical diameter (unless stated). However, the semi-analytic model can be applied to any smooth functions describing pore and particle geometry. TRPS of non-spherical particles is gaining prominence, with recent examples including self-assembled cylinders [105], nanorods [99], viruses [55], bacterial chains [96], rod-shaped bacteria [99], RBCs [50] and specific aggregates [106]. Particle aggregates are especially important for assays in which a target molecule causes two or more particles to stick together. Two recent assays $[107,108]$ have attributed pulses to dimers, trimers, and tetramers when the pulse height has been an integer multiple of the height for individual particles, as initially modelled for superparamagnetic particle aggregates (Figure 2.3) [83, 
106]. A method has been suggested for determining whether particles are aspherical based on their pulse shapes [50]. This method allows for differentiation between spherical and non-spherical particles and has been used to measure non-spherical particles such as RBCs and bacteria [99].

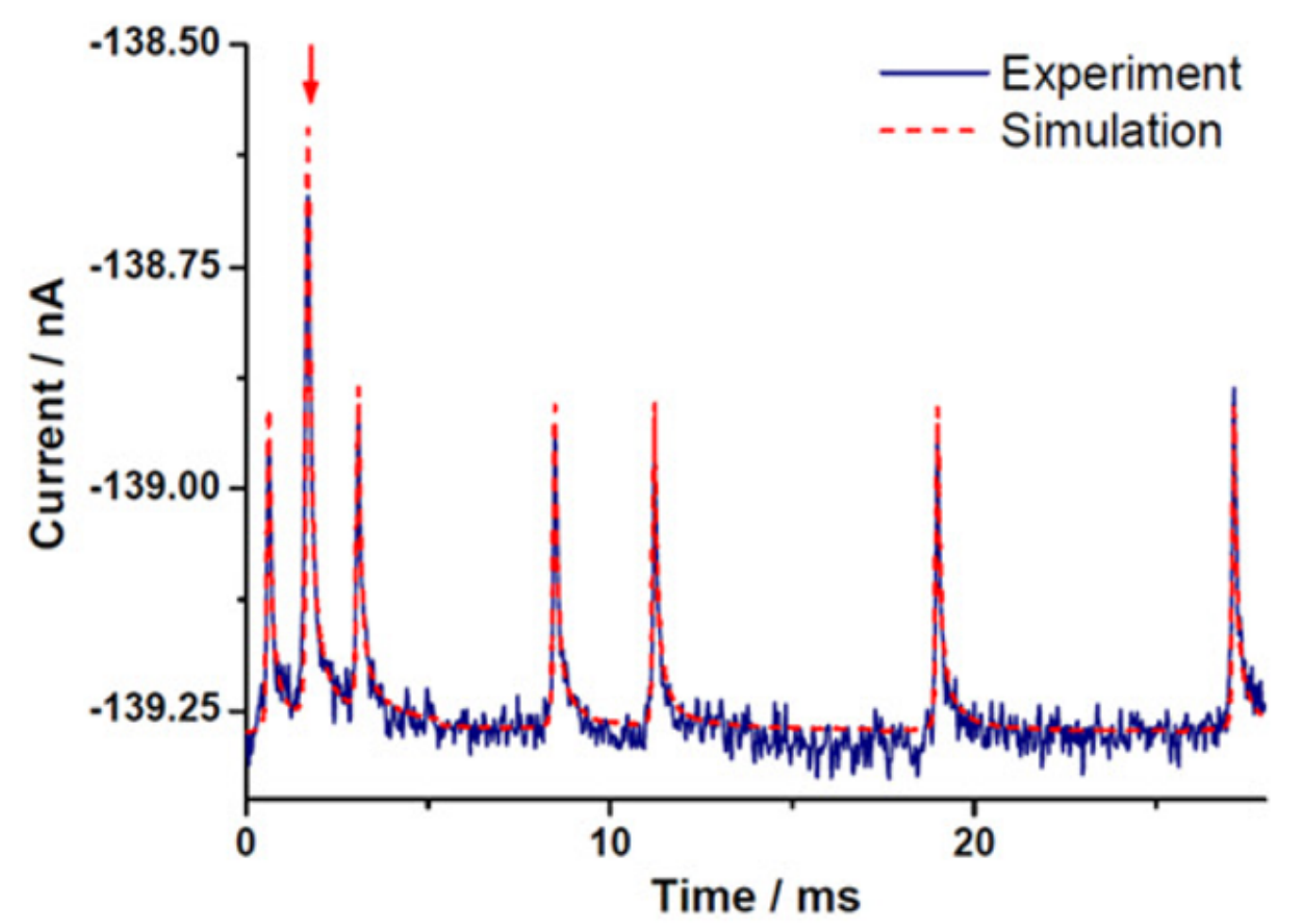

Figure 2.4: Resistive pulses for $1 \mu \mathrm{m}$ spheres from an experiment (blue) and the semi-analytic model (red). The pulse indicated by the red arrow is modelled as a dimer of spheres, whereas other pulses correspond to individual particles. Reproduced from Willmott et al. [83]. 


\section{Charge Measurement}

Measurements of $\zeta$-potential, the most widely used measure of colloidal electronic charge, were first reported using particle-by-particle resistive pulse measurements by DeBlois et al. [19]. These researchers used polycarbonate nanopores to obtain $\zeta$-potentials for populations of $91 \mathrm{~nm}$ latex spheres and a range of viruses, calculating the particle mobility from the measured resistive pulse rate. Ito et al. $[58,59,109]$ later reported $\zeta$ potentials for $60 \mathrm{~nm}$ carboxylated polystyrene particles based upon the total duration of individual resistive pulses, measured using carbon nanotubebased Coulter Counters. Both of these groups worked with cylindrical pores of fixed size. These studies have been improved upon in recent analyses of resistive pulse-based $\zeta$-potential measurement $[56,110]$.

TRPS can be used to find a particle's $\zeta$-potential $\left(\zeta_{\text {particle }}\right)$. Considering Equation 2.3, the electrophoretic mobility can be calculated by measuring the particle velocity, then finding the electric field and pressure-driven flow using the semi-analytic model. To enable calculation of $\zeta_{\text {particle }}$, the $\zeta$-potential of TPU $\left(\zeta_{\text {pore }}\right)$ has been measured $[47,111]$. TRPS measurement of $\zeta_{\text {particle }}$ was first achieved [47] by finding the pressure at which $\mathbf{J}=0$ 


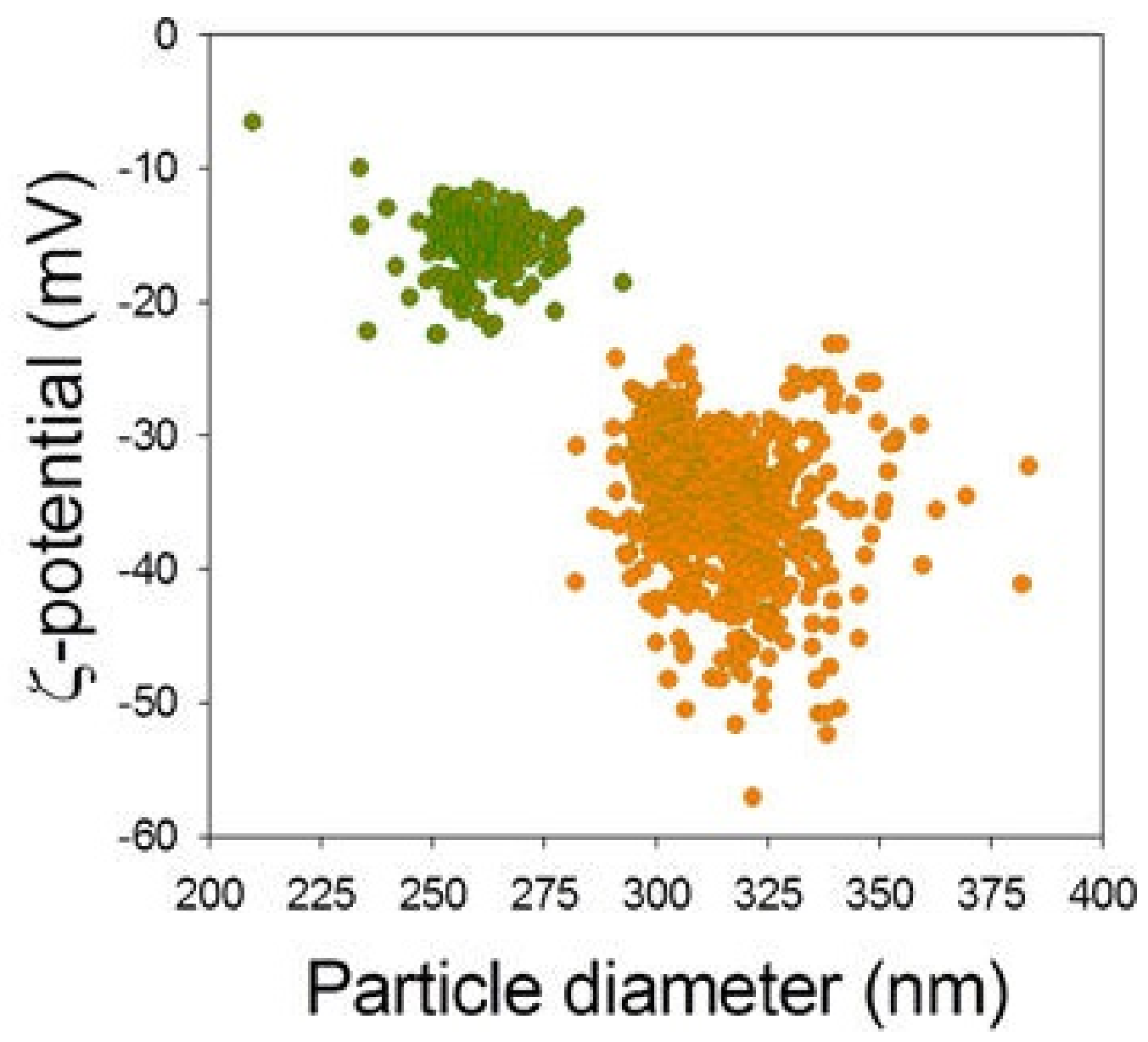

Figure 2.5: Two populations of $300 \mathrm{~nm}$ carboxylated polystyrene particles are distinguished from a mixture. The scatter plot includes particle-byparticle measurements of size and $\zeta_{\text {particle }}$, the latter calculated from fullwidth half-maximum (FWHM) durations. Adapted from [46]. 
in Equation 2.2. Pressure can be varied either continuously or in discrete steps, and $\mathbf{J}=0$ can be identified using either the greatest value of the fullwidth half-maximum (FWHM) duration or the minimum pulse rate [111]. When $\mathbf{J}$ is non-zero, velocity profiles can be built for individual pulses [82], yielding particle-by-particle $\zeta_{\text {particle }}$ values using either geometric inputs [46] (Figure 2.5) or calibration particles [48]. Recently, a multi-point iteration of the velocity profile method has been used to measure the $\zeta$ potential of particles modified with biological scaffolds [112-114]. TRPS charge measurements are covered in more detail in Chapters 4 and 5.

Comparable RPS charge measurements include a method based on transit time alone [58] as well as more detailed calculations [56, 74]. TRPS has been used to find $\zeta_{\text {particle }}$ for an emulsion [14], liposomes [115, 116] and DNA conjugated nanoparticles [117]. Pulse rates and durations can also be used to infer changes in surface functionalization without explicit calculation of charge $[42,53,118-121]$. For example, injectable drug carriers often use polyethylene glycol (PEG) to avoid adhesion to biological material before reaching a tumour, and TRPS can be used to observe the addition of PEG to particle surfaces [122]. 


\subsubsection{Comparison with Other Techniques}

Colloidal characterization techniques such as TRPS, DLS [123] and SEM and TEM each employ different physical principles. They are often used together to study the same sample, with each experiment providing distinct information. Two quantitative studies $[12,124]$ have compared the same emerging techniques (TRPS, nanoparticle tracking analysis (NTA), and differential centrifugal sedimentation (DCS)) with more established techniques (TEM and DLS). Anderson et al. [12] measured particle size distributions for monodisperse and multiple population solutions of 220$410 \mathrm{~nm}$ polystyrene particles (Figure 2.6), whereas Bell et al. [124], who also used scanning mobility particle sizing, measured monodisperse samples of five Stöber silica particle sets with diameters of 100-400 nm. Both studies produced TRPS size distributions consistent with TEM. Another quantitative comparison [103] included multimodal size distributions of polystyrene beads measured alongside urinary EVs, and compared TRPS with TEM, NTA, and conventional and dedicated FC. For the polystyrene beads (>100 nm), TRPS had the lowest sizing error after TEM and dedicated FC, with the latter technique performing well due the refractive index uniformity of polystyrene particles. In concentration measurements 
(approximate range $10^{9}-10^{10} \mathrm{~mL}^{-1}$ ), TRPS gave good results for polystyrene standards, and results for EVs agreed with values obtained using NTA, with FC giving a lower result and TEM suffering from losses during sample preparation. Recently, Varga et al. [125] used EVs to compare TRPS, NTA, EM, small angle X-ray scattering, size exclusion chromatography and DLS. Modal values were consistent $(\sim 130 \mathrm{~nm})$ across the techniques, but the size distributions varied. A recent study compared TRPS and 8 other particle characterisation techniques using poly(isobutylcyanoacrylate) nanoparticles decorated with dextran. It found the single particle measurement techniques, including TRPS, were superior [126]. There are similar comparative studies that have not involved TRPS [102, 127].

Eldridge et al. [50] made a qualitative comparison of TRPS with DLS, NTA, DCS, FC, suspended microchannel resonators and EM in the context of drug delivery applications. The physical nature of each technique was summarized, and measureable ranges of sample size, concentration and volume were tabulated. Similarly, a recent review article examines TRPS for nanomaterials in nanodiagnostics [128]. Similar qualitative comparisons have specifically referred to measurement of EVs [129] and solid lipid nanoparticles [130]. Heider and Metzner's review of virion measure- 


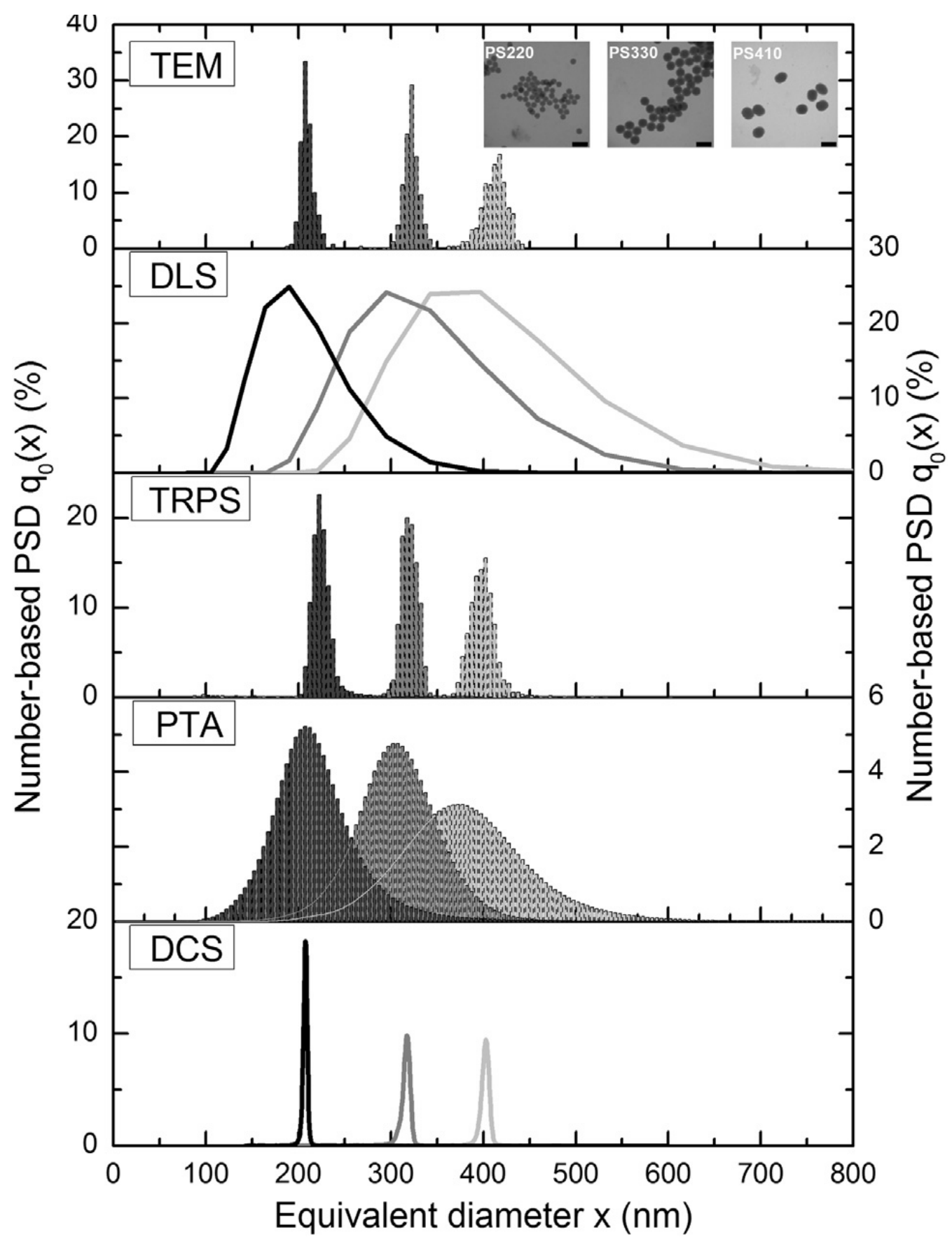

Figure 2.6: Comparison of TRPS with other particle characterization techniques (PTA is equivalent to NTA) showing polystyrene particle size distributions for three solutions. Reproduced from Anderson et al. [12]. 
ment methods [55] considered TRPS, NTA, advanced field flow fractionation (FFF), and a virus counter method involving fluorescent labelling, along with time-consuming biological techniques. Advantages of TRPS for virions include efficient size measurement to determine aggregation state, the ability to measure low particle concentrations by applying pressure, and (along with NTA and FFF) charge measurement capability. Virus shape cannot be determined in great detail using TRPS, while the small instrument footprint and the possibility for specificity are advantages. On the other hand, some viruses are currently beyond the lower size capabilities of TRPS, and refinement of raw biological samples is important for all techniques.

Other experimental comparisons involving TRPS have typically been restricted to a few measurements, the relative merits of which may not extend beyond each set of experimental conditions. Perceived advantages of TRPS have included the accessible ranges of particles [124], electrolytes $[124,131]$ and measured quantities [103, 131, 132]. Advantageous sample volume (tens of $\mu \mathrm{L}$ ) [96, 133], cost and portability [131] and accuracy [97] have also been noted. The accessible size range is not unlimited [98, 103, $124,134]$, but can be extended by the use of multiple pores. Discussions of 
measurement speed (a few minutes per measurement, following training) $[96,103]$ and associated pore clogging [103] require experimental context. For example, sample preparation [135] and classification is an issue for complex biological media regardless of the measurement technique [133]. In concentration measurements, TRPS has been more accurate than FC and phase contrast microscopy [44, 132], and better than optical density measurements in comparison with the plating method for bacteria [96]. However, inconsistencies have been demonstrated in a comparative study of 200 nm EVs and liposomes [116] and studies affected by sample contamination $[55,96,136]$.

There is relatively little comparative work involving charge measurement. Like TRPS, commonly used DLS-based techniques derive $\zeta_{\text {particle }}$ from electrophoretic mobility (Equation 2.3). In a recent comparison using $\sim 200 \mathrm{~nm}$ polystyrene particles [111], the typical difference between $\zeta$-potentials obtained using TRPS and DLS was $15 \%(<5 \mathrm{mV})$, with an experimental error of $\sim 10 \%$ for each technique. In another recent study [114] both TRPS and DLS were used to measure the $\zeta$-potential of $\sim 100 \mathrm{~nm}$ protein coated silica nanoparticles. The techniques found very similar surface charges. 


\subsubsection{Distinct Characteristics of TRPS}

TRPS avoids difficult sample preparation and experimental artefacts associated with EM [74], although the electrolyte can cause unwanted aggregation [130]. Measurements are independent of optical properties [125] such as particle labelling, knowledge of refractive index, or refractive index contrast. TRPS analyses assume that the particle is an ideal insulator, and measurement protocols can require calibration [103]. Experimental parameters can be varied to optimize TRPS data resolution, and two recent studies have done this systematically $[116,137]$. When particles fall outside the size detection thresholds for a particular pore, summary statistics [137] and concentration measurements [116, 137] are necessarily affected. This highlights the advantage of using the population mode as a statistical description particle size distributions, and the benefits of TRPS comparisons under identical experimental conditions. TRPS provides particleby-particle data which is beneficial for resolving multi-modal $[12,53,98$, $103]$ and high dispersity $[103,130,138]$ distributions (especially in comparison with DLS). This data can be used to calculate any central value or spread statistic which enables the broadening study in Chapter 7, and can be transformed for direct comparison with ensemble average data [14]. 
Many considerations apply more generally when selecting a colloidal characterization technique. For example, the accessible ranges of particle concentration and size are important, as well as the volume and type of solution. The overall importance of user knowledge regarding instrument settings and data handling has been noted $[116,125]$. Care is required regarding the specific type of size measurement [124] and theoretical differences in size distributions [12, 102]. For biological solutions, sample preparation is especially critical. Lane et al.'s [135] TRPS study, which used liposomes to systematically compare isolation protocols based on ultracentrifugation, sedimentation reagents and density gradient, is important in this respect. There are opportunities to develop traceable uncertainty analyses for most emerging techniques, including TRPS [125]. Coumans et al. [137] recently used TRPS to obtain 102 repeat size distribution measurements for urinary vesicles, highlighting the importance of studying reproducibility. Finally, the experimental design should always be considered relative to the specific research question. 


\begin{tabular}{|c|c|c|c|c|c|c|c|}
\hline Ref. & Particle Material & Surface Function & Indicative Size $^{a}(\mathrm{~nm})$ & Particle Concentration $\left(\times 10^{9} \mathrm{~mL}^{-1}\right)$ & Analyte & Analyte Concentration $^{b}$ & Detection \\
\hline \multicolumn{8}{|c|}{ Assays } \\
\hline \multirow[t]{2}{*}{ [106] } & $\mathrm{Au} / \mathrm{Ni}$ rods & $\operatorname{Avidin}(\mathrm{Ni})$ and PEG $(\mathrm{Au})$ & 1230 long $\times 100$ diameter & 0.24 & Biotin & $\sim 100 \mathrm{fM}$ & Mean $\triangle \mathrm{I}$, FWHM \\
\hline & & Aptamer $(\mathrm{Ni})$ and PEG $(\mathrm{Au})$ & 1100 long $\times 300$ diameter & 0.09 & PDGF & $\sim 100 \mathrm{fM}$ & Mean $\Delta \mathrm{I}$, FWHM \\
\hline \multirow[t]{3}{*}{ [107] } & SPBs & Avidin & 3000 & 0.5 & Biotin & $\sim 1 \mathrm{pM}-1 \mathrm{nM}$ & Mean $\Delta \mathrm{I}$, mono- and multimer fractions \\
\hline & & Avidin & 1000 & 6 & Biotin & $\sim 1 \mathrm{nM}$ & Monomer fraction \\
\hline & & Streptavidin & 300 & 10 & Biotin & $\sim 1 \mathrm{nM}$ & Monomer fraction \\
\hline [108] & $\mathrm{Au}$ & $\mathrm{DNA}^{c}$ & 25 & 60 & DNA & $5.0 \mathrm{pM}$ & Pulses observed \\
\hline [118] & $\mathrm{Au}$ & Citrate & 50 & 45 & PNA & $5 \mathrm{nM}$ & Size-duration scatter plot \\
\hline [139] & $\mathrm{Au}$ & (i) Avidin (ii) DNA & (i) 30 (ii) 55 & (i) 4.2 (ii) 13 & DNA & 530 copies & Size threshold \\
\hline [140] & Magnetic beads & Protein $^{d}$ & 1000 & $6 \times 10^{-4}$ & $\mathrm{RBCs}$ & $\sim 10^{7} \mathrm{~mL}^{-1}$ & Mean size, size distribution \\
\hline Other & & & & (Specified units) & (Mechanism) & & \\
\hline \multirow[t]{2}{*}{ [14] } & Soy bean oil & $\beta$-lactoglobulin & 150 & $10 \mathrm{wt} \%$ oil & $\sim 120$ days & & Size mode, mean \\
\hline & & $\beta$-lactoglobulin & 150 & $10 \mathrm{wt} \%$ oil & Salt & & Size mean, median \\
\hline [83] & SPBs & Carboxylate & 1000 & $0.4 \times 10^{9} \mathrm{~mL}^{-1}$ & Bar magnet & & Size distribution, pulse rate, time between pulse \\
\hline \multirow[t]{4}{*}{ [131] } & Carbon Nanohorns & & $1-2$ & $0.5-50 \mu \mathrm{g} \mathrm{mL}^{-1}$ & In cell media & & Size distribution \\
\hline & Carbon black & & 14 & $0.5-50 \mu \mathrm{g} \mathrm{mL}^{-1}$ & In cell media & & Size distribution \\
\hline & $\mathrm{CeO}_{2}$ & & $7-25$ & $0.5-50 \mu \mathrm{g} \mathrm{mL}^{-1}$ & In cell media & & Size distribution \\
\hline & $\mathrm{Ni}$ & & 60 & $0.5-50 \mu \mathrm{g} \mathrm{mL}^{-1}$ & In cell media & & Size distribution \\
\hline [141] & Liposomes & EPC-3-based ${ }^{f}$ & 250 & $5 \mathrm{mM}$ lipids & $\sim 1$ hour & & Size distribution \\
\hline [142] & Wine proteins & & $200-4000^{g}$ & $100 \mathrm{mg} \mathrm{L}^{-1}$ & Heating & & Size distribution, concentration \\
\hline
\end{tabular}

Table 2.3: Summary of TRPS studies in which particle aggregation has been detected. $\mathrm{PEG}=$ polyethylene glycol, $\mathrm{PDGF}=$ platelet-derived growth factor, $\mathrm{PNA}=$ peptide nucleic acid, $\mathrm{SPBs}=$ superparamagnetic beads, $\mathrm{RBCs}=$ red blood cells. ${ }^{a}$ As available: mode, mean or manufacturer's specification. ${ }^{b}$ Order of magnitude estimate indicated by $\sim$ unless limit of detection claimed in original publication. ${ }^{c}$ Two types of bead with different DNA ends (18 and 100 base pairs (bp)) matching the ends of the target. ${ }^{d}$ The protein annexin-V binds to phosphatidylserine found on the surface of RBCs after eryptosis. ${ }^{e}$ This study pointed out that the mean particle size gives higher sensitivity to aggregation than the mode when most beads are not aggregated. $f$ Hydrated egg phosphatidylcholine in lipolysis medium. ${ }^{g}$ Range of aggregate sizes formed from molecular protein. 


\subsection{Applications}

\subsubsection{Diagnostics and Genomics}

This Section focusses on the use of TRPS to study DNA, aptamers and other molecules which support specific binding interactions. The goal is often a diagnostic, sensing or monitoring assay at low concentrations of target. Bead aggregation is one common method for detecting the presence of a target, and understanding particle aggregation is of wider importance for TRPS. TRPS studies of particle aggregation have been summarised in Table 2.3.

\section{DNA}

As noted in Chapter 1 , interest in the wider field of nanopore science has been driven by potential DNA sensing applications. TRPS is no exception, as the first description of size-tunable pores [41] involved detection of double stranded (ds) DNA molecules each consisting of 2686 base pairs (bp). Controlled gating of these molecules was reported (Figure 2.7a), with resistive pulses observed only at relatively high stretch. The par- 
ticular geometry of the pore used in this study allowed detection of DNA molecules, but due to the relative ease of tunable pore fabrication at larger length scales, no subsequent TRPS studies have reported detection of single molecules.

Subsequently, TRPS has been used for on-bead DNA sensing in which evidence for molecular interactions is provided by detecting changes in particle size or surface charge due to functionalization or aggregation. Firstly, TRPS was used to simply distinguish $220 \mathrm{~nm}$ organosilica nanoparticles modified with $\lambda$-DNA (48 kbp dsDNA, $16 \mathrm{~nm}$ long) from unmodified particles [53]. The modified particles produced an increased pulse duration resulting from reduced surface charge, without an observable increase in particle size. Low et al. studied non-specific aggregation of citrate capped gold nanoparticles in the presence of a mixed base peptide nucleic acid (PNA, 20 bp single-stranded ssDNA) [118]. The TRPS size measurement for single nanoparticles $(\sim 50 \mathrm{~nm})$ agreed with DLS and TEM results. On addition of PNA, TRPS indicated aggregates with diameters up to 125 $\mathrm{nm}$ in addition to charge neutralisation (increased pulse duration). The lowest PNA concentration at which aggregation was inferred was $5 \mathrm{nM}$, whereas $50 \mathrm{nM}$ was required using DLS. 

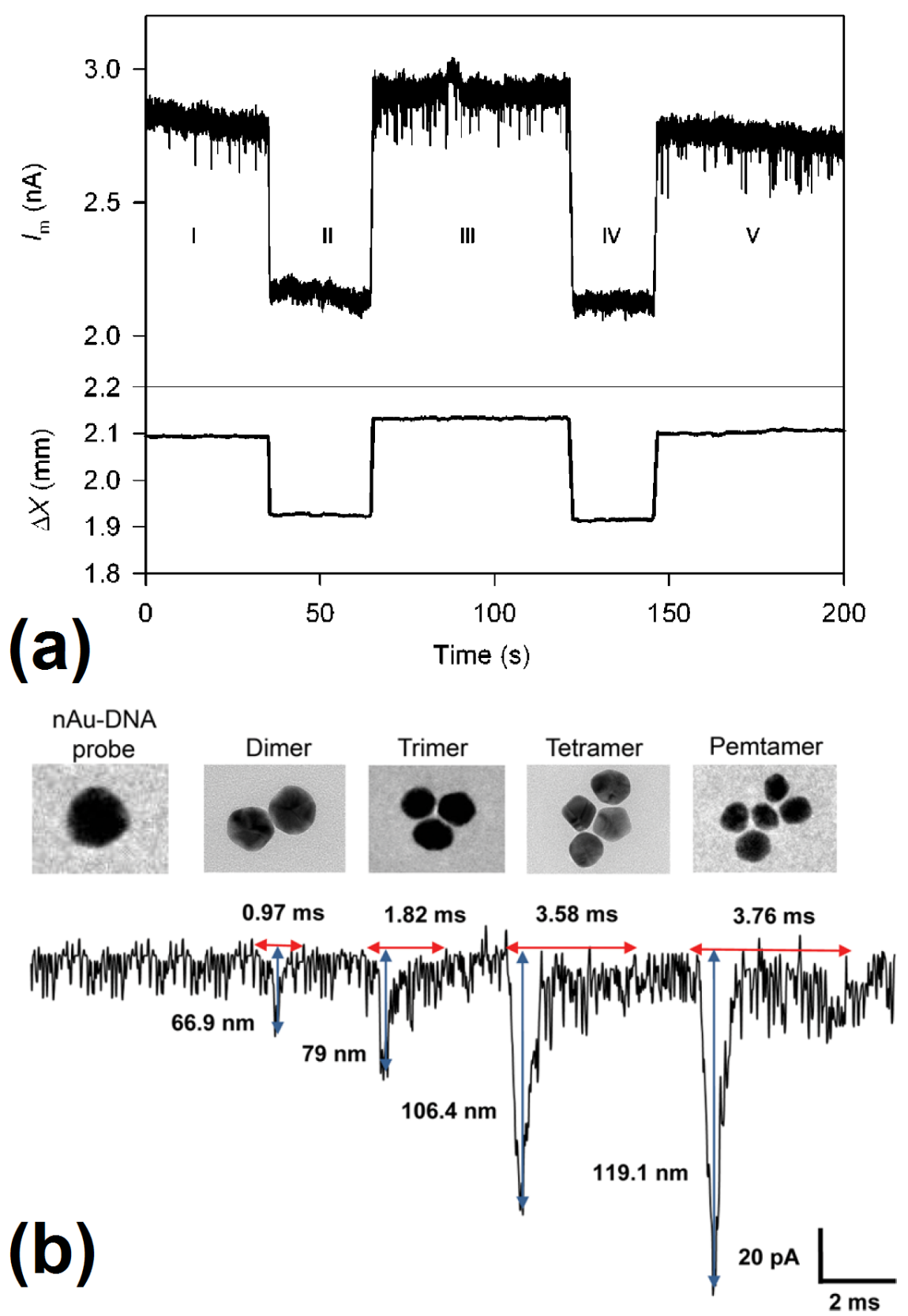

Figure 2.7: TRPS experiments using DNA. (a) Gating of 2.7 kbp dsDNA molecules, reproduced from Sowerby et al. [41]. Traces show the stretch applied to a membrane (lower), and ionic current (upper) as a function of time. Zones labelled I-V are delineated by abrupt changes corresponding to adjustment of $X$. (b) Distinguishing multimers of DNA-aggregated $25 \mathrm{~nm}$ Au nanoparticles. TEM images (upper) demonstrate aggregates as observed in the ionic current trace (lower). Arrows and labels indicate the mean baseline duration and modal magnitude. The latter is scaled to effective spherical diameter. Adapted from [108]. 
Specific DNA interactions were first studied with TRPS by Booth et al. [117], who functionalized dextran-based magnetic beads with 23 bp DNA complementary to a target. There was no significant change in the modal size recorded before $(109 \mathrm{~nm})$ and after $(106 \mathrm{~nm})$ the addition of $0.01 \mathrm{nM}$ target DNA to $2 \times 10^{11} \mathrm{~mL}^{-1}$ beads, but the modal FWHM duration was reduced from $0.95 \mathrm{~ms}$ to $0.68 \mathrm{~ms}$. This indication of increased particle charge was verified using TRPS charge measurements [47] in which the $\zeta$-potential increased from $-11 \mathrm{mV}$ to $-17 \mathrm{mV}$.

Two studies [108, 139] have used a TRPS sensing strategy in which two types of gold nanoparticle have induced aggregation in the presence of specific target DNA. In both cases individual nanoparticles were too small to be detected, so resistive pulses indicated the presence of the target as aggregation increased particle size and therefore resistive pulse magnitude. Ang and Yung [108] prepared two sets of $25 \mathrm{~nm}$ gold particles with DNA fragments (18 and $100 \mathrm{bp}$ ) complementary to the different ends of a target. With the target present, the observed aggregates could be classified as dimers, trimers, tetramers and pentamers on the basis of pulse magnitudes (Figure 2.7b, see also [107]). Aggregates were not observed when the target sequence was altered by a single base. Target concentrations 
from $5.0 \mathrm{pM}$ to $2.5 \mathrm{nM}$ were near-linearly correlated with aggregate detection rate, suggesting a method for quantifying target DNA concentration. Yang et al. [139] used $30 \mathrm{~nm}$ and $55 \mathrm{~nm}$ gold nanoparticles functionalized with avidin and a thiol-DNA probe respectively. The target DNA, a 340 bp gene from biofilm-forming methicillin-resistant $S$. aureus, underwent loop-mediated isothermal DNA amplification so that it would bind to both types of nanoparticle, inducing aggregation. Using arbitrary size thresholds, the authors quoted a limit of detection of 530 DNA copies within a processing time of 2 hours. The technique was tested against a negative control, and also showed good specificity against a strain of S. aureus which does not carry the same gene.

TRPS has been used for label-free counting of individual strands of synthetic Pseudomonas aeruginosa DNA [143]. Padlock probes, which ensured specific capture of the target, were bound to $1 \mu \mathrm{m}$ magnetic beads and subjected to rolling circle amplification, producing large coils of ssDNA attached to the beads. When beads were mixed with DNA at a 10:1 ratio, the number of strands could be quantified using the increased baseline pulse duration. This method was demonstrated using a pulse duration detection threshold, with a lower detection limit near to $10 \mathrm{zmol}$ of 
DNA ( $\sim 6000$ molecules) in $10 \mu \mathrm{L}$ of electrolyte (i.e., $1 \mathrm{fM}$ ). The authors noted that the method is more sensitive than similar fluorescence based methods, with a total assay and analysis time under 1 hour. More recently a novel polymer-peptide hybrid system for gene transfection was synthesized. This formed approximately $100 \mathrm{~nm}$ particles when complexed with DNA, the size of which were measured using TRPS [144].

Blundell et al. [112] measured the $\zeta$-potential of DNA modified particle using a newly developed multi-point velocity profile method (see Chapters 4 and 5 for more details). As the ssDNA concentration added increased from 10-210 $\mathrm{nM}$, the $\zeta$-potential of the particles decreased from -4 to $-16 \mathrm{mV}$. Different lengths and types of complementary DNA were also added to see the effect of dsDNA on particle charge. The types and lengths added included full complementary DNA, middle binding DNA (which only created a small dsDNA section in the middle of the strand), end binding (dsDNA section at the end), and overhang binding (dsDNA at the end which overhangs to give extra ssDNA). Addition of any of the complementary DNA types caused the $\zeta$-potential to become more negative. Longer incubation time also resulted in more negative $\zeta$-potentials. The overhang binding DNA gave the most negatively charged particles 
(up to a $-8 \mathrm{mV}$ change), followed by the full complementary, the middle binding, and finally the end binding DNA which gave the smallest decrease in $\zeta$-potential.

\section{Aptamers and Other Specific Interactions}

Aptamers are short, single-stranded pieces of DNA or RNA that are developed to have specific binding affinity for a target molecule [106]. The selectivity, stability and cost of aptamers are attractive for sensing applications $[106,107,119]$. In that context, it is unsurprising that TRPS has been used to investigate the use of aptamers more often than other specific interactions. Platt et al. [106] were the first to use TRPS to study both an aptamer capture probe and the strong protein-ligand, avidin-biotin interaction. The detection strategy involved aggregation of cylindrical nanorods (diameter $300 \mathrm{~nm}$, length $2-4 \mu \mathrm{m}$ ) in the presence of the target protein, platelet-derived growth factor. Each rod was a 'barcode' of gold and nickel segments. By appropriate functionalization of the barcode, the orientation of aggregates could be controlled, and changes in pulse magnitude and FWHM were specific to the resulting aggregate shape (Figure 2.8). Detection in the femtomolar range was enabled by the superparamagnetic 
properties of the Ni-containing rods.

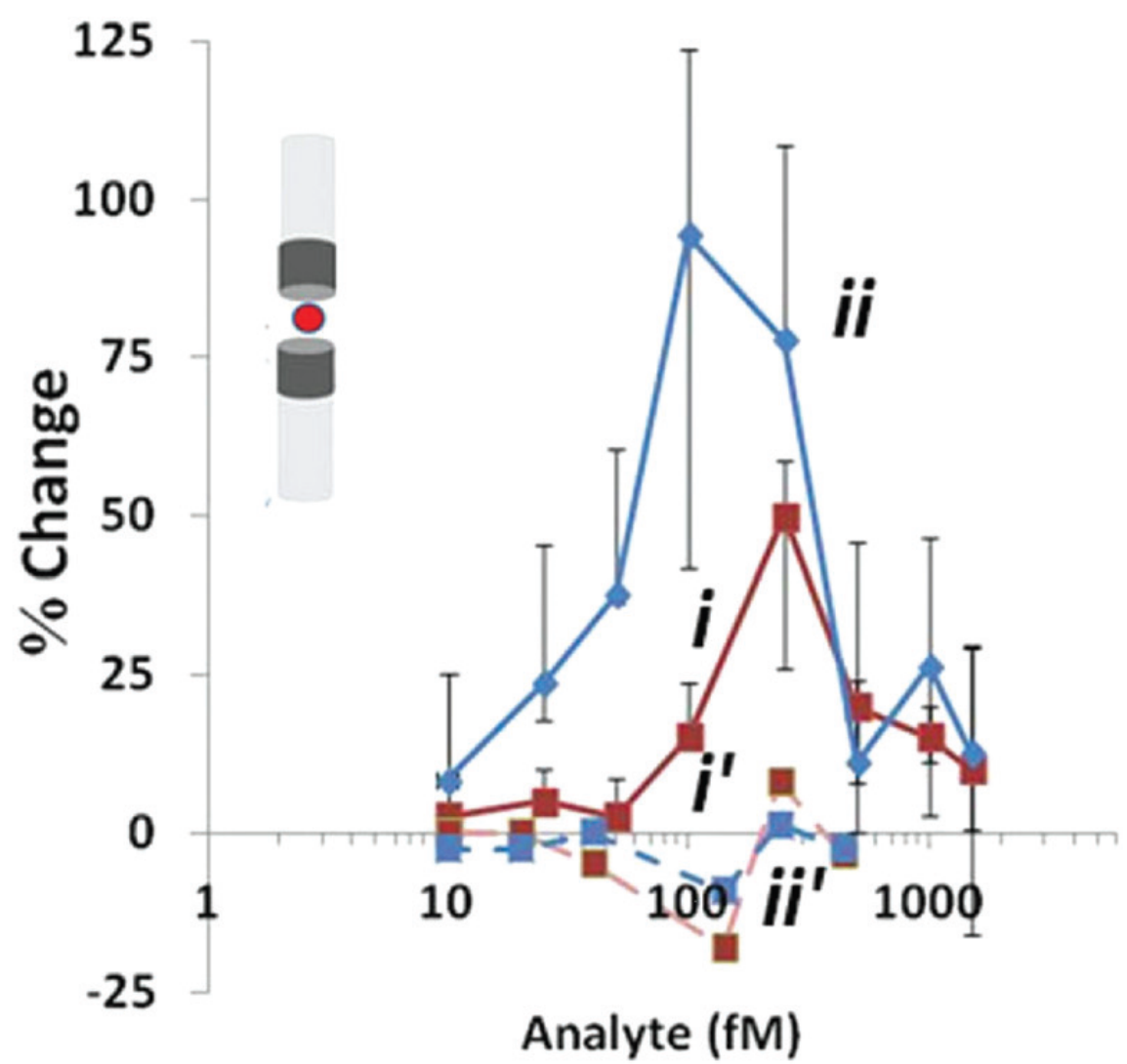

Figure 2.8: Nanorod aggregation in the presence of biotinylated bovine serum albumin. Only the Ni segments of AuNi barcoded rods (dark grey in inset schematic) were functionalized with avidin, inducing end-to-end aggregates. The changes in resistive pulse magnitude $(i, r e d)$ and FWHM duration (ii, blue) are compared with a control analyte (dashed lines). Reproduced from Platt et al. [106].

Billinge et al. [107] subsequently used TRPS to study aggregation of 3 $\mu \mathrm{m}$ superparamagnetic beads (SPBs) coated with avidin in the presence of picomolar biotinylated bovine serum albumin. Aggregation, as indicated 
by increased pulse sizes and fewer pulses, reduced at higher analyte concentrations $(\sim 1 \mathrm{nM})$ due to saturation of the bead surface with analyte. This 'hook' effect, so-called because of the form of data plots, can produce a false negative result and is a general feature of aggregation assays. This aggregation assay was also studied with variations in bead concentration, bead size, binding capacity, and with a bar magnet present. The same team reported on detection of the protein thrombin [145] using beads modified with three different aptamers. In this study, the decrease in pulse rate due to charge screening was the best indicator of target presence, and pulse rates were studied in real time (over $\sim 3$ minutes) after thrombin (0.1-1000 $\mathrm{nM})$ was added to the beads $\left(3 \times 10^{9} \mathrm{~mL}^{-1}\right)$ in the TRPS fluid cell. The change in pulse rate allowed calculation of dissociation constants for the aptamers, and the values obtained were consistent with literature. Variations in assay performace between the three aptamers were attributed to conformational changes when binding to the target. More recently, Billinge et al. [146] showed that the use of two different sized beads allowed for the detection of two separate proteins in a one-step measurement. In contrast to previous studies which saw a diminishing pulse rate with increasing analyte concentration, this study found that at a negative voltage a more conventional positive correlation could be made between 
analyte concentration and pulse rate. This was due to the analyte carrying a positive charge at the $\mathrm{pH}$ used and therefore responding to a negative bias.

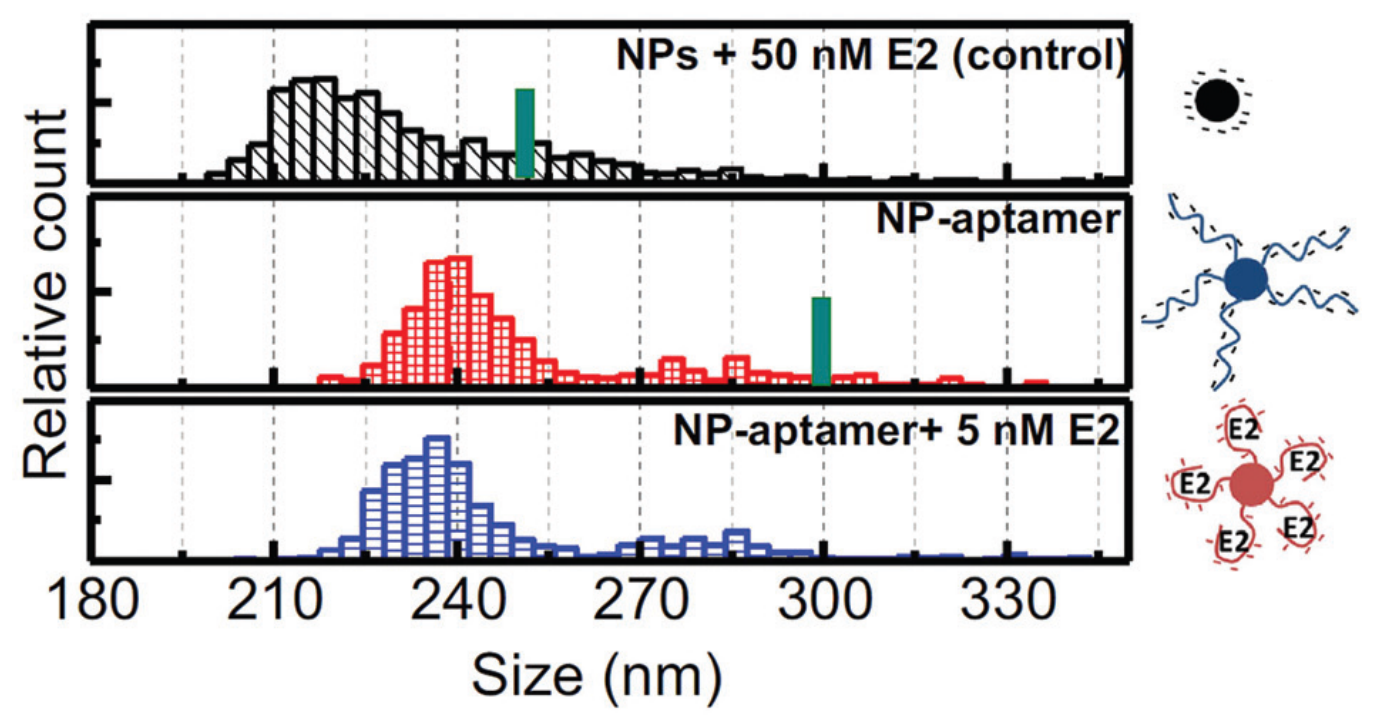

Figure 2.9: TRPS size histograms with accompanying schematic diagrams for carboxylated polystyrene nanoparticles in the presence of a target (upper), the same nanoparticles coupled to aptamers (middle), and modified nanoparticles with the target present (lower). Green bins indicate the average of DLS size distributions. Adapted from Alsager et al. [119].

Alsager et al. also used an on-bead aptamer based detection strategy, but without aggregation [119]. An aptamer for the target $17 \beta$-estradiol was tethered to $217 \mathrm{~nm}$ polystyrene nanoparticles, and TRPS was used to study functionalized nanoparticles $\left(5.2 \times 10^{10} \mathrm{~mL}^{-1}\right)$ exposed to target concentrations between 5-150 nM. Resistive pulse magnitudes increased with the attachment of the aptamer, then decreased on addition of the tar- 
get due to conformational change (Figure 2.9), in qualitative agreement with DLS. Nanoparticle functionalization resulted in increased pulse duration, which was inconsistent with DLS charge measurements, and was attributed to increased steric drag in the pore constriction. Pulse FWHM values also increased when the target was added, in agreement with the DLS trend, and consistent with charge screening by the target. The assay did not discriminate between the target and compounds from the same steroid family, but excellent discrimination was observed for bisphenol compounds. Overall, TRPS assays using aptamers have revealed that changes in particle size and charge can be detected, but are not always predictable due to the unique three-dimensional binding conformation between the aptamer and its target.

Further specific interactions studied using TRPS include detection of aggregates formed by $1 \mu \mathrm{m}$ beads and RBCs [140]. Aggregation was enabled by the specific affinity between a protein on the beads and the outer lipid bilayer of RBCs following eryptosis, which was instigated either by increasing osmolarity or using a potential anti-cancer drug. Lai et al. [147] used TRPS size distributions to observe binding of antibody-loaded protein microparticles to a target molecule. Protein microparticles were sus- 
pended in the fluid cell above a pore, and the mean particle diameter increased from $1041.5 \mathrm{~nm}$ to $1212.6 \mathrm{~nm}$ when the target (mouse immunoglobulin $\mathrm{G}(\operatorname{IgG})$ ) was introduced. No size change was observed on the addition of rabbit IgG.

\subsubsection{Extracellular Vesicles}

TRPS is being applied widely to studies of EVs [148, 149], which are lipid bilayer vesicles secreted by most human cells. EVs play a role in inter-cell communication, in gene delivery and as disease biomarkers [98, 148-150]. Their concentration and composition can be altered by pathological conditions [150], and they can carry microRNA (miRNA). There is currently a need to classify and understand the roles of particular EVs. By one definition [150, 151], EVs of endosomal origin are termed exosomes (generally 30-100 nm), whereas EVs originating from the plasma membrane are termed microvesicles (100-1000 nm). EVs are also sometimes referred to as microparticles. However, classification is challenging, because a raw sample of bodily fluid (e.g. blood, urine or lymph) contains a complex, high dispersity mixture of sub-cellular particles and proteins. This problem has produced high demand for new measurement tools [102], and 
consequently TRPS has recently featured in several reviews and comparisons of EV measurement techniques [103, 125, 129, 133, 137, 152-158]. EVs have been measured from $60 \mathrm{~nm}[54,135,159]$ through to $1 \mu \mathrm{m}$ [132], and multiple pores have been used to handle highly disperse distributions [103, 132], but lower size limits for TRPS do not currently extend to the smallest EVs $[125,137]$ as noted in comparisons with EM (Figure 2.10a) [98, 134, 160]. Protocols for EV collection, isolation, handling, and storage for TRPS, including a size exclusion column produced by Izon Science [161], are in development [135, 152], and analysis of protein content is not currently enabled [129].

TRPS sensitivity and stability specific to the analysis of exosomes was investigated by Anderson et al. [163]. They noted that exosome samples often have a substantial percentage of their size distribution falling below the calculated sensitivity cut-off (defined as the diameter of a particle causing a $0.05 \mathrm{nA}$ blockade at any given settings) for TRPS measurement, and suggested that multiple techniques should be used to characterize exosome samples. Suggested instrument settings for exosome measurement included a root mean squared (RMS) noise of 6-9 pA, low membrane stretch, high applied voltage (as long as RMS noise stays low), 0.05 

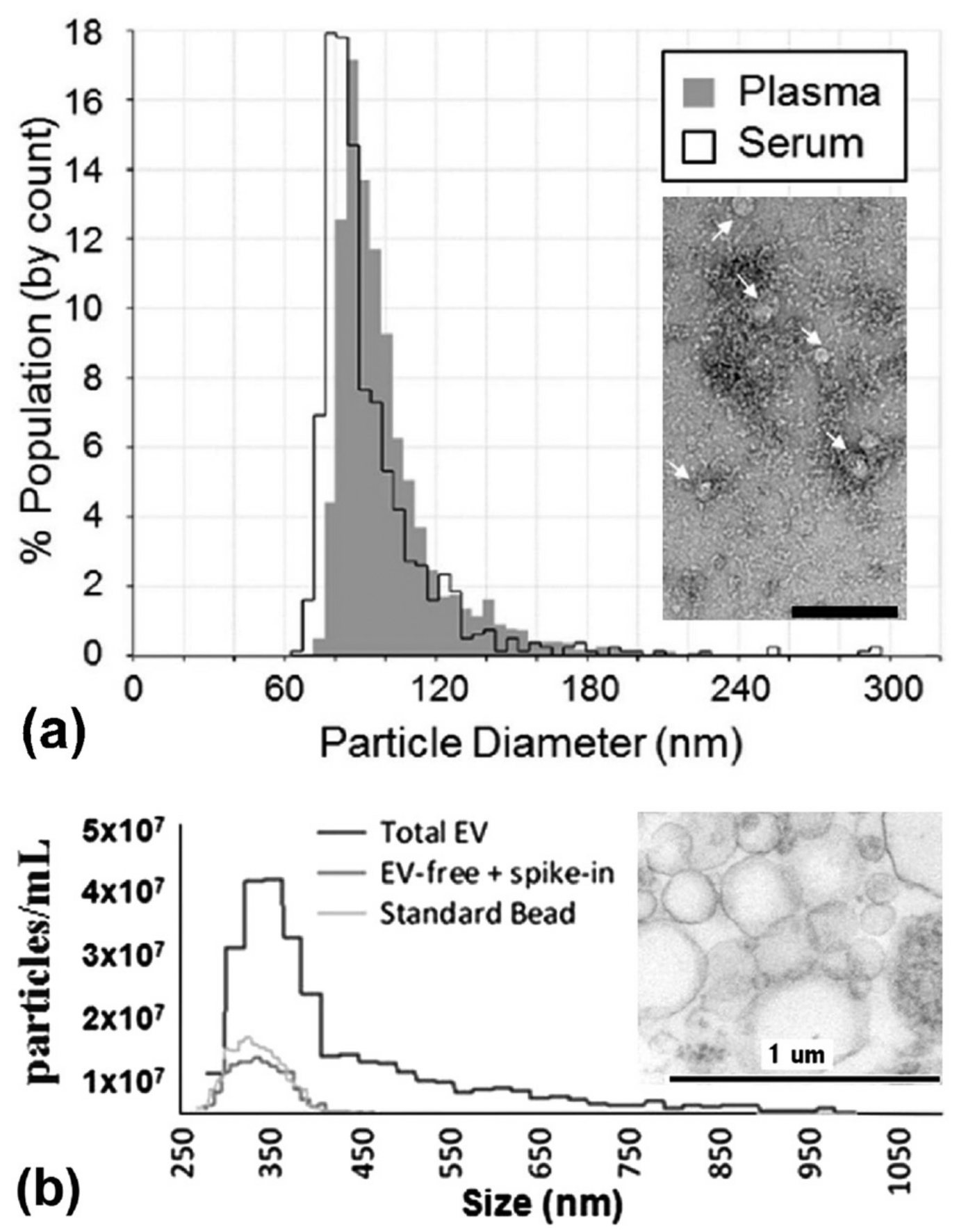

Figure 2.10: TRPS experiments using EVs. (a) Size distributions for exosomes from blood plasma and serum. Inset, TEM of plasma exosomes (denoted by arrows, scale bar $200 \mathrm{~nm}$ ). Adapted from Cheng et al. [160]. (b) TRPS size distributions and TEM (inset) for EVs in cell-free supernatants derived from acute lymphoblastic leukemia cells. In the TRPS data, exclusion of EVs in one solution was confirmed by spiking the solution with $400 \mathrm{~nm}$ reference beads. Adapted from Szabó et al. [162]. 
$\%$ Tween-20 surfactant, pressure below $11 \mathrm{~cm} \mathrm{H}_{2} \mathrm{O}$ for NP100 pores and $<15 \mathrm{~cm} \mathrm{H}_{2} \mathrm{O}$ for NP200, and a particle rate of 100-200 minute ${ }^{-1}$. Prefiltration and pore orientation were not seen to have an effect. The remainder of this Section covers TRPS studies of exosomes isolated from particular cell types, demonstrating a wide range of measurement roles and application areas.

Urinary vesicles occur naturally at relatively high concentration with low contamination. In their comparative study, van der Pol et al. [103] used urine, centrifuged to remove cells and diluted in phosphate buffered saline (PBS). Size distributions for these vesicles were obtained using pores of two sizes (Figure 2.3). Coumans et al. [137] also used urinary vesicles for their study of TRPS reproducibility (see Section 2.1.5), while de Vrij et al. [98] studied EVs isolated from urine as well as blood plasma and pleural fluid. Their samples all produced TRPS size distributions with more than $95 \%$ of particles in the range $150-400 \mathrm{~nm}$. Cheng et al. measured size distributions for exosomes derived from urine [164] and blood [160] in studies aiming to develop collection and processing methods miRNA sequencing. A standardized ultracentrifugation protocol was used to isolate exosomes, and TRPS size distributions provided evidence that the miRNA 
yield from exosomes isolated from urine and resuspended in PBS was superior to samples from the usual medium, cell-free urine.

TRPS has been used to analyse the size and concentration of microparticles in blood before and after apheresis, a treatment for removing cholesterol [165]. Apheresis did not alter the modal microparticle size, while the concentration dropped when measured using pores of target diameter 200 $\mathrm{nm}$ and NTA, but was unchanged when using pores of target diameter $100 \mathrm{~nm}$. These differences illustrate the importance of size thresholds and sample preparation for biological fluids. Burnouf et al.'s review of methods for studying platelet microparticles (PMPs) [133], the most abundant microparticles in blood, included a TRPS size distribution for $\sim 400 \mathrm{~nm}$ PMPs in platelet-poor plasma. PMPs are $0.1-1 \mu \mathrm{m}$ phospholipid-based fragments shed from platelets when they undergo activation, stress, or apoptosis. PMP concentration has been measured in a number of recent studies [166, 167]. Higher-than-normal PMP concentration has be associated with some clotting and inflammatory diseases. Thus, reducing the number of microparticles in plasma using filtration has been investigated. TRPS was used to show that the filtering successfully lowered the concentration of PMPs [166]. 
EVs derived from cancer cells give information about the type of cancer [168] and have been shown to play a role in tumour growth and spread [169]. Szabó et al. [162] analysed centrifuged supernatant from a culture of leukaemia cells in a study concerned with the effects of EVs on human monocytes in the presence of recombinant human tumour necrosis factor. As well as measuring the EV concentration and size distributions (Figure 2.10b), TRPS played a role confirming that a nominally EV-free medium was indeed free of microvesicles. Patko et al. [134] obtained a size distribution in the range $200-300 \mathrm{~nm}$ in a study of leukaemia-derived EVs binding to the extracellular matrix.

The role of an enzyme (capase-3) in production of EVs from breast cancer cells has also been investigated [132]. In cells transfected with capase3, the concentration of EVs increased approximately 5-fold, most significantly in the size range 400-600 nm from an overall measured range of 100$1000 \mathrm{~nm}$. Here, TRPS was preferred over FC as the latter does not give particle size information and underestimated the particle concentration. EVs from cancer cells undergoing treatment play a role in radiation induced non-targeted effects. TRPS was used to measure the size and concentration of EVs from irradiated breast cancer cells and showed an increase in 
concentration after irradiation while EV size did not change substantially. De Vrij et al. also measured tumor cell-derived EVs between 100 and 200 $\mathrm{nm}$ in diameter $[98,170]$ and were able to quantify EV secretion numbers per cell [170]. Murine melanoma cells were used to investigate 3 different exosome isolation methods. Exosome size, measured with TEM and TRPS, was found to be mostly between $50-200 \mathrm{~nm}$ for all methods [171].

EVs derived from mesenchymal stem cells (MSCs) can be used as delivery vehicles. TRPS has been used to measure size distributions for EVs that could deliver enzymes to combat Alzheimer's disease (150-200 nm) [172], EVs which accelerate skeletal muscle regeneration [173], EVs that assist with recovery after traumatic brain injury $(116 \pm 49 \mathrm{~nm})$ [174] and anti-cancer miRNA to tumour cells $(60-180 \mathrm{~nm}$, with the major peak at 65-75 nm) [159]. In the latter study, TRPS suggested that the number of secreted nanoparticles (including exosomes) increased following transfection of synthetic miRNA into MSCs. Elsewhere, EVs from endometrial epithelial cells were compared with EVs found in uterine fluid and mucus in a study of implantation in the uterine cavity [175]. The similarity of size distributions from different sources $(100-500 \mathrm{~nm}$ with a mode of $\sim 100 \mathrm{~nm})$ suggested that epithelial EVs are released into the uterine cavity. Lipo- 
somes, which are synthetic spherical compartments enclosed by a phospholipid bilayer [176], can be used as a model for EVs. Lane et al. [135] demonstrated that model liposomes had a similar size distribution $(\sim 100$ $\mathrm{nm})$ to exosomes derived from serum-free cell culture media at concentrations between $1.5 \times 10^{9}$ and $3 \times 10^{11} \mathrm{~mL}^{-1}$. Maas et al. [116] compared analyses of tumour cell-derived EVs and $212 \mathrm{~nm}$ liposomal EV mimics at $3.3 \times 10^{13} \mathrm{~mL}^{-1}$ using TRPS, NTA and high-resolution FC. TRPS results were obtained for various pore geometries, applied voltages, buffers, calibration particle sets and particle dilutions, and emphasized the importance of reproducible measurement through understanding and control of experimental settings.

In the first TRPS study of bacteria-derived EVs, Bogomolny et al. [177] measured the size and concentration of uropathogenic Escherichia coli (E. coli) EVs. A modal diameter of $124 \pm 3 \mathrm{~nm}$ and lower concentration bound of $7.4 \times 10^{9} \mathrm{~mL}^{-1}$ were measured. EVs produced by sulfur-reducing, hyperthermophilic archaea, Thermococcus onnurineus $\mathrm{NA}^{\mathrm{T}}$ were characterized using TEM and TRPS finding particle sizes in the range 80-210 nm and 120-550 $\mathrm{nm}$ respectively [178].

Emerging reports on EV research [179] suggest that there are other 
TRPS studies in progress concerning EVs derived from fibroblasts, marrow, white blood cells, and from humans as well as animal models. Very recently published studies examine adipocyte-derived EVs [180] and neurological EVs [181].

\subsubsection{Nanomedicine}

\section{Drug Delivery}

Many nanoparticle formulations are being explored in order to provide new drug administration methods that are non-invasive, targeted, and provide controlled medication release [50, 182, 183]. Physical properties (including size, charge and concentration) can affect the circulation time, localization, cellular uptake, drug release profile and toxicity of nanoparticles in vivo [184]. Therefore accurate characterisation is necessary to ensure effectiveness and quality control. Regulatory compliance has been highlighted by a recent study [115] which demonstrated TRPS measurement of reporting statistics for Caelyx, a commercial liposome-based drug treatment. TRPS yielded a narrower size distribution than DLS, and was also used to study liposomal size differences, concentration, charge (therefore 
surface properties), and aggregation during the freeze-thaw process.

Liposomes, a major class of drug delivery nanoparticles, are usually prepared by extrusion of lipid through a filter, producing a particle size range close to the diameter of the filter pores [97, 185]. For the first liposomes studied using TRPS [185], particle size distributions $(\sim 200 \mathrm{~nm})$ and pulse rates were measured as a function of the jaw width and applied pressure. TRPS size distributions have been used to monitor the stability of two liposome dispersions by looking for signs of aggregation in particle size distributions. In the first case, tumour-targeting polymer-liposome complexes $(\sim 150 \mathrm{~nm})$ were more stable than two more standard liposome types when exposed to protein treatment [120]. In the second, the size distribution of soy phosphatidylcholin (SPC) liposomes (100-160 nm) did not change significantly following incubation in a medium approximating the gastro-intestinal track, whereas liposomes based on hydrated egg phosphatidylcholine formed aggregates up to $1 \mu \mathrm{m}$ in diameter over 60 minutes [141]. Yang et al. also studied SPC liposomes [97], extruded using filters approximately 100, 200 and $400 \mathrm{~nm}$ in size. TRPS was able to take measurements at more than 20 times greater dilution of phospholipid than DLS. TRPS and DLS yielded similar size measurements for the smaller two 
samples, while inconsistencies obtained for the largest sample were attributed to DLS uncertainties caused by size dispersity. Incorporation of a photochromic material (spiropyran) into the lipid layers produced a photosensitive liposome, for which the $\zeta$-potential could be switched using exposure to UV-light. Switching the $\zeta$-potential to positive values allowed for better cell adhesion. Correlation of pulse duration to $\zeta$-potential was used to measure the $\zeta$-potential of the liposomes [186].

Similarly, a TRPS size distribution for lyophilisomes (biocapsules made from water soluble proteins, with the defining factor that there is no need for amphiphilicity [187]) over the range 700-1600 nm gave a mean diameter of $1214 \mathrm{~nm}$ [138], in comparison with a higher DLS value of $1695 \mathrm{~nm}$ [187].

Particles which release their drug in response to a particular chemical or physical stimulus are a promising development in drug delivery. The drug can be delivered to a tumour specifically, and in high concentrations. For example, Burgess and Porter $[188,189]$ used TRPS to study phase-shift nanoemulsions (PSNEs), which release their drug when an ultrasound stimulus converts nanoemulsion drops to nanobubbles. The size of PSNEs (mean diameter $178.3 \mathrm{~nm}$ and concentration $5.3 \times 10^{11} \mathrm{~mL}^{-1}$ 
in the 2013 study and modal diameter $203 \mathrm{~nm}$ and concentration $2.03 \times$ $10^{11} \mathrm{~mL}^{-1}$ in the 2015 study) is important as they must be able to move through blood vessel walls into neighbouring tissue. TRPS has more commonly been used to study particles which release their payload in the slightly acidic conditions ( $\mathrm{pH}$ 5.0) found within a cell. This is the case for extracellular-matrix targeting liposomes (above) [120], hydrogel particles of diameter $\sim 1500 \mathrm{~nm}$ which degrade below $\mathrm{pH} 7.4$ [190], and polymeric nanoparticles (200-250 nm) which burst at $\mathrm{pH} 7.4$ and in the presence of gastrointestinal enzymes [191].

Colby et al. [192] used TRPS to study 'expansile' nanoparticles, which SEM suggests are 20-200 nm in diameter near $\mathrm{pH} 7.4$, but expand to 200$2000 \mathrm{~nm}$ at $\mathrm{pH} 5.0$ as their polymer matrix breaks down. The proportion of particles measured by the larger of two pores increased from $<1 \%$ to $90 \%$ over 5 days, consistent with an increase of measured average diameter from $876 \pm 259 \mathrm{~nm}$ to $1339 \pm 516 \mathrm{~nm}$ between days 3 and 5 . Pulse durations for expanded nanoparticles became larger and more irregular when the applied stretch was decreased (Figure 2.11), suggesting that the soft expanded nanoparticles squeeze through the pore. The same group recently investigated stabilizing the expansile nanoparticles with a PEG- 
ylated lipid coating. The qNano was used to confirm that the new coating did not affect swelling properties [193]. These experiments represent an initial foray towards the use of tunable pores to mechanically interact with soft nanoparticles, as also suggested in recent RPS reports using static membranes [74, 194, 195]. The deformability of soft particles can make TRPS measurements challenging as they have a tendency to spread and block the pore as opposed to squeeze through it. Despite this Bachman et al. [196] measured the size of ultra low crosslinked soft microgel particles and compared it to that of more highly crosslinked microgels. Recently, deformable cationic microgels have also been investigated [197].

Nitric oxide (NO)-releasing nanoparticles have emerged as a wound healing enhancer and a novel antibacterial agent that can circumvent antibiotic resistance. The size of NO-releasing poly(lactic-co-glycolic acid) (PLGA)-polyethylenimine nanoparticles was measured using TRPS as well as SEM. Particle sizes were $175 \pm 35 \mathrm{~nm}$ and $179 \pm 25 \mathrm{~nm}$ for particles with and without NO respectively, compared with $166 \pm 46 \mathrm{~nm}$ and 162 $\pm 19 \mathrm{~nm}$ from SEM [198]. The same group also studied dexamthasone phosphate (DP) loaded PLGA nanoparticles, where DP selectively targets activated microphages for alleviation of inflammatory disease. These par- 

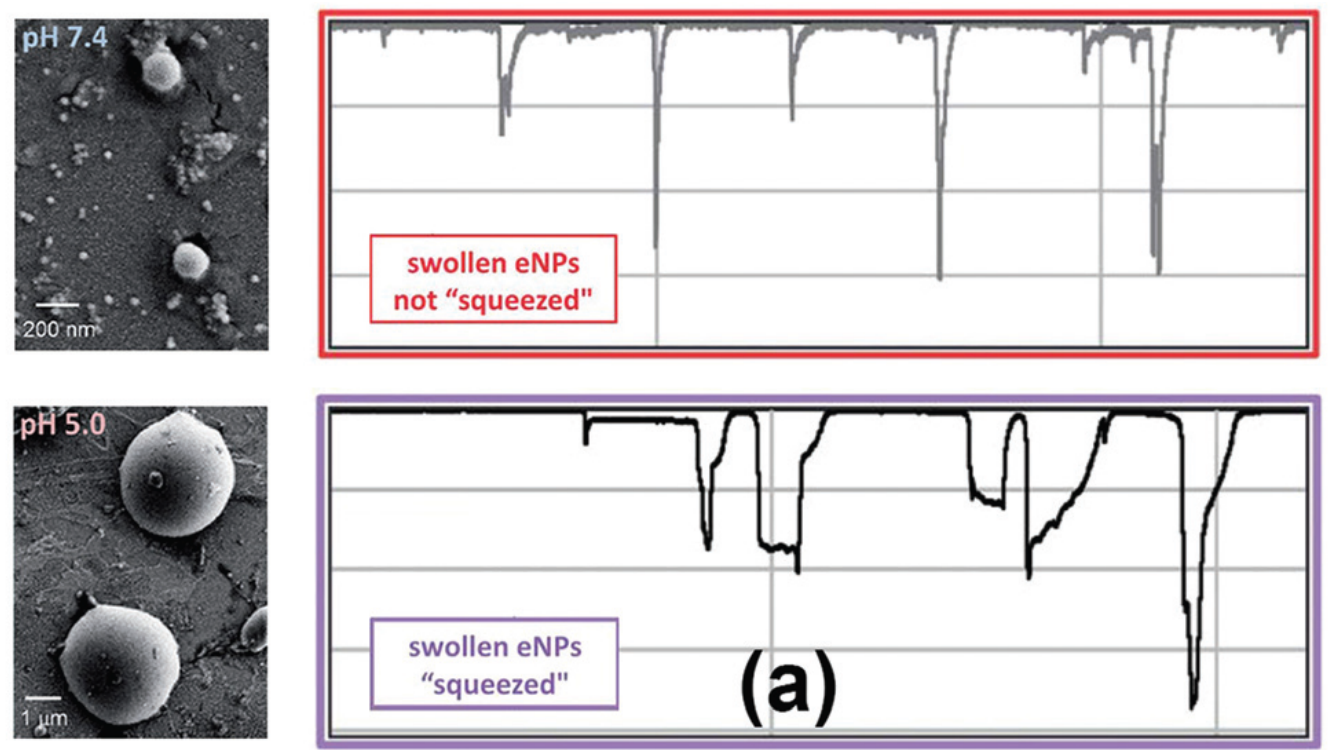

Figure 2.11: Expansile nanoparticles used for drug delivery are much larger at lower $\mathrm{pH}$ (SEM images, left). TRPS experiments using the expanded particles can 'squeeze' the particles by reducing the pore size, resulting in longer pulse durations in current-time traces (right). Adapted from ref. [192] 
ticles were $121 \pm 27 \mathrm{~nm}$ and $128 \pm 30 \mathrm{~nm}$ before and after conjugation with folic acid, respectively [199]. Hwang et al. [200] undertook a study of the cytotoxicity and inflammatory response caused by the addition of cationic surfactants to lipid and polymeric drug delivery particles in which particle concentration was measured using the qNano.

Magnetically loaded particles also show promise for drug delivery because they can be directed to specific sites using an external magnet [201]. TRPS has been used to measure the size distributions of lipid particles that were small enough for administration by inhalation $(<5 \mu \mathrm{m})$, and contained superparamagnetic iron-oxide nanoparticles (SPIONs) along with a model drug, budesonide [202]. These particles had a mean diameter of $2.2 \mu \mathrm{m}$ (mode $1.6 \mu \mathrm{m}$ ) without SPIONs, rising to $2.8 \mu \mathrm{m}$ (mode $1.8 \mu \mathrm{m}$ ) when SPIONs were included. Results obtained using DLS $(3.2 \mu \mathrm{m}$ and 2.9 $\mu \mathrm{m}$ respectively) were again larger due to a small population of large aggregates. The range of drug delivery systems analysed using TRPS now includes solid lipid nanoparticles [130] and fluorocarbon droplets which could simultaneously act as contrast agents [203, 204].

Microbubbles are another potential drug delivery vehicle. Manta et al. [205] optimised TRPS measurements for microbubbles, which included 
short measurement times and multiple pores, for both positive and negatively charged microbubbles. Measurements were comparable to optical microscopy but allowed for the measurement of smaller microbubbles. As well as a solo delivery agent, microbubbles can be used to stabilize other polymeric drug delivery particles, for example PEG-coated poly(butyl cyanocrylate) particles - the concentration of which was measured by TRPS and NTA and found to be $10^{12}$ to $10^{14} \mathrm{~mL}^{-1}$.

\section{Nanotoxicology}

The potential for TRPS in nanotoxicology has been demonstrated by Pal et al. [131], who measured aggregates of engineered nanomaterials - carbon nanohorns, carbon black, $\mathrm{CeO}_{2}$ and Ni nanoparticles. Size and concentration measurements were carried out in fetal bovine serum, and although TRPS and DLS particle size distributions were similar, TRPS was able to distinguish two modes in the size distribution. A key outcome for in vitro toxicity assessment was that particle characterization uncertainties were less significant than the uncertainty in relating an administered dose to the dose delivered to a cell. TRPS has also been suggested as a measurement tool for particles released from nano-enabled products [206]. 


\subsubsection{Phages, Viruses, Bacteria and Algae}

Bacteriophages and viruses, which range in size from tens to hundreds of nanometers, and bacteria, typically a few micrometers in size, can all be cultured in solutions suitable for TRPS. In these fields, TRPS has mostly been used for basic characterization of size and concentration. There is a particular need to complement or replace plating, the laborious gold standard for bacterial concentration measurements [96]. The potential for TRPS in this area was first demonstrated using lambda phage [51], which infects E. coli. The shapes of resistive pulses appeared to be consistent with the head-tail geometry of the phages (Figure 2.12a). Another phage measurement [207] concerned the Serratia entomophila anti-feeding prophage (Afp), which causes amber disease in New Zealand grass grub. A particle concentration of $4.1 \times 10^{7} \mathrm{~mL}^{-1}$ within the size range $140-165 \mathrm{~nm}$ was deemed to correspond to the Afp.

The first TRPS measurement of a virus [43] used purified samples of the spherical adenovirus virion, suspended in PBS, yielding a size distribution with a modal value of $96.5 \pm 15 \mathrm{~nm}$, compared with a literature value of 70-90 nm. The narrow histogram peak (Figure 2.12b) suggested 


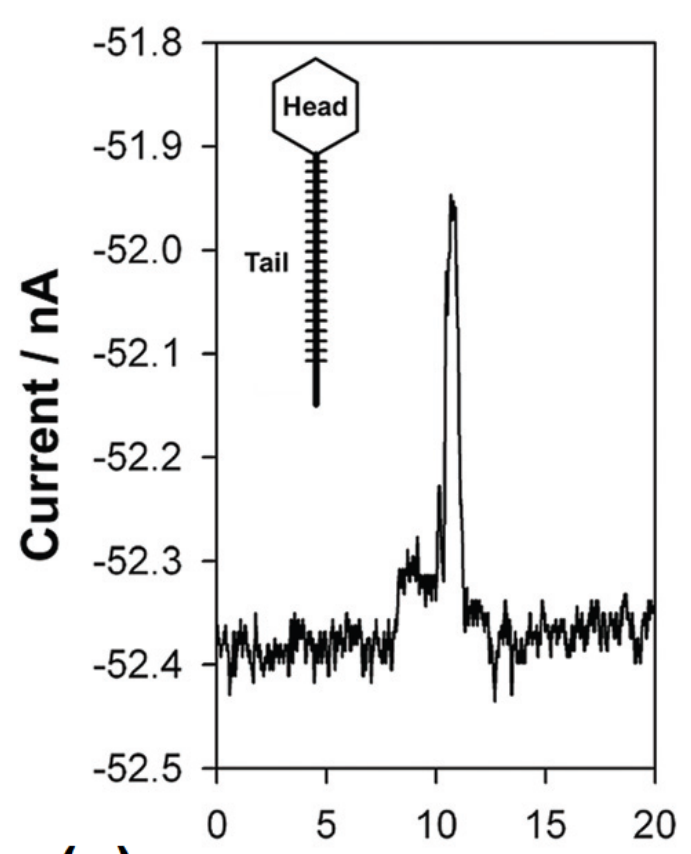

(a)

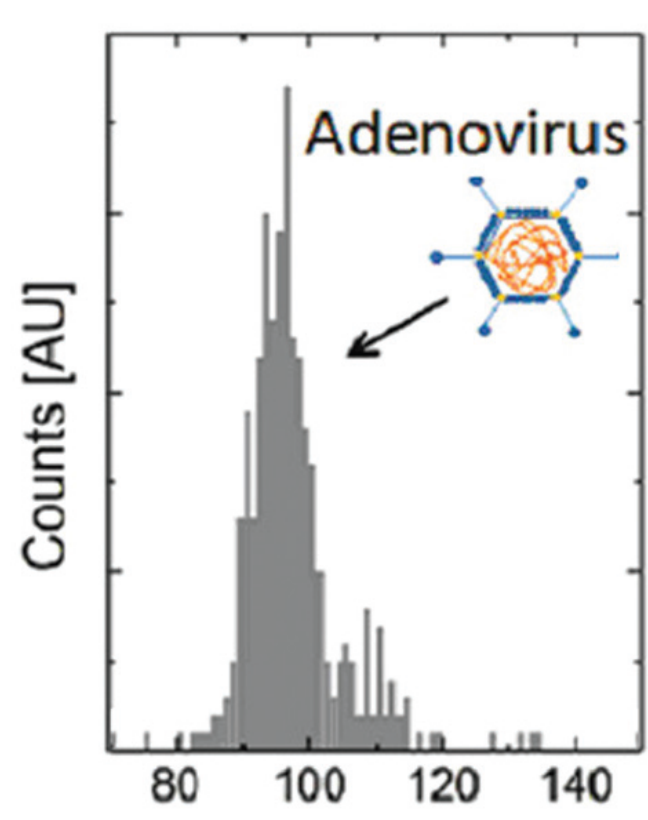

(b) Virus Diameter [nm]

Figure 2.12: (a) Resistive pulse for a lambda phage (length $\sim 230 \mathrm{~nm}$ ), represented by the inset schematic diagram. The shoulder of the pulse prior to the maximum resistance change suggests that the phage tail passed through the pore prior to the head. Reproduced from Willmott et al. [51]. (b) TRPS size histogram for adenovirus particles, with inset schematic of the virus. Adapted from Vogel et al. [43] 
that the virions did not aggregate, an important trait for potential gene therapy applications. Farkas et al. [136] used TRPS to study rotavirus (diameter $\sim 75 \mathrm{~nm}$ ), the most common intestinal virus. A size exclusion chromatography method for sample purification was paired with TRPS to prevent virus count overestimation, a common problem for rotavirus. On the other hand, measurements based on protein and DNA content caused concentration overestimates $\left(>10^{12} \mathrm{~mL}^{-1}\right)$ for lentivirus $(80-100 \mathrm{~nm})$ due to contaminating molecules outside the virions [55]. The TRPS value (8 $\times 10^{10} \mathrm{~mL}^{-1}$ ) was compared with an infectivity titer, indicating that only $0.001 \%$ of particles were infectious. Elsewhere, Arjmandi et al.'s mass measurement technique [104] was applied to inactivated virions in $75 \mathrm{mM} \mathrm{KCl.}$ Human immunodeficiency virus was measured to be $198 \pm 15 \mathrm{~nm}$ in size with density $1.2 \pm 0.1 \mathrm{~g} \mathrm{~cm}^{-3}$ and Epstein-Barr virions were $170 \pm 13$ $\mathrm{nm}$ at a density of $1.7 \pm 0.2 \mathrm{~g} \mathrm{~cm}^{-3}$. These results were comparable with measurements made using rigid etched silicon nanopores and other methods. Recently Akpinar et al. [208] compared TRPS and TEM for measuring the size and concentration of the bullet shaped vesicular stomatitis virus. TRPS was able to quantify concentrations down to $10^{7} \mathrm{~mL}^{-1}$ providing a 50-fold larger measurement range than TEM as well as more reproducible counts. Equivalent particle diameter was estimated from TEM and com- 
pared to TRPS, giving $111.8 \mathrm{~nm}(n=14)$ and $107.8 \mathrm{~nm}(n=1540)$ respectively. Gaudin et al. [209] used the qViro to verify the accuracy of a size sorting method applied to Junin virus.

TRPS size and concentration measurements were first applied to bacteria by Roberts et al. [44], who studied the marine cyanobacterium Prochlorococcus, and Baculovirus occlusion bodies. TRPS size distributions for Prochlorococcus (range 300-1200 nm with a mean of $\sim 650 \mathrm{~nm})$ and Baculovirus $(\sim 1$ $\mu \mathrm{m})$ agreed with previous reported values. Bacterial concentrations (6.0 $\times 10^{8} \mathrm{~mL}^{-1}$ and $9.9 \times 10^{7} \mathrm{~mL}^{-1}$ respectively) were consistent for different users on different days, and lower (by $6 \%$ and $17 \%$ respectively) than measurements using a FC haemocytometer and phase contrast microscopy. Accurate determination of Baculovirus concentration is vital for insecticide applications. Allen et al. [96] measured cell size and concentration simultaneously for strains of Bacillus subtilis and E. coli during colony growth. TRPS concentrations correlated more closely with colony plating than optical density measurements. Particle volumes between 1.5-10 fL were obtained for each sample, consistent with previous work and microscopy, and the cultures incubated with more glucose present produced slightly larger values. Bennett et al. [210] found that the bacteria in probiotics used 
in four dairy feed products had similar sizes, in the range 800-2000 nm, with concentrations between $4.4 \times 10^{9}-1.2 \times 10^{10} \mathrm{~mL}^{-1}$.

In studies of water-borne pathogens which can be harmful to humans, Chung et al. [121] compared a wild type strain of Francisella tularensis with a mutant, while Pang et al. [211] investigated potential surrogates for Cryptosporidium parvum (C. paroum). In the former study, the mutants ( 750 nm) were slightly larger than wild type bacteria, with longer pulse durations suggesting that they also have less negative charge. In the latter, TRPS confirmed that the size of protein-modified polystyrene microspheres $\left(\sim 4.9 \mu \mathrm{m}\right.$, and concentration $\left.2 \times 10^{6} \mathrm{~mL}^{-1}\right)$ was similar to $C$. parvum oocysts (reproductive cysts containing a zygote). The qMicro was used to measure the size of microalgae while investigating their lipid content and accumulation [212].

\subsubsection{Other}

Food and beverage emulsions can be structurally similar to particles such as EVs and liposomes, consisting of small capsules stabilized by surfactant molecules. TRPS has been used to characterise soy bean oil droplets 
stabilised by $\beta$-lactoglobulin [14], an emulsion model for milk. The emulsion was refrigerated and monitored over four months, during which the modal droplet size increased from $150 \mathrm{~nm}$ to more than $200 \mathrm{~nm}$. The size distribution dynamics inconclusively suggested that the dominant growth mechanism involved migration of oil molecules, as in Ostwald ripening, whereas aggregation induced by addition of salt was more consistent with flocculation and coalescence. The surface charge of the emulsion droplets was also measured using the variable pressure method [47]. Average $\zeta-$ potentials were calculated using both resistive pulse rate $(-18.9 \mathrm{mV})$ and duration $(-21.8 \mathrm{mV})$ data, and were compared with a DLS measurement $(-27.6 \mathrm{mV})$. Beyond emulsions, Gazzola et al. studied protein haze [142], a wine quality defect. Aggregation of five wine proteins was analysed following different treatments containing phenolics and/or polysaccharide, by measuring aggregate sizes from approximately $200 \mathrm{~nm}$ to $4000 \mathrm{~nm}$, and concentrations from $2.0 \times 10^{5}$ to $7.5 \times 10^{6} \mathrm{~mL}^{-1}$.

SPBs are widely used in biotechnology, because a magnetic field can transport SPBs independently of other interactions [213]. This is useful for separation, concentration and aggregation, and as discussed above SPBs have been used to assist drug delivery [201], separation in DNA assays 
$[117,143]$, and efficient separation and aggregation in other targeted assays [106, 107]. Two studies have focussed exclusively on understanding magnetic particle transport in TRPS $[83,91]$. In the first, $1 \mu \mathrm{m}$ SPBs were used to show how TRPS can be used to detect aggregation [83]. Upon application of a magnetic field, frequently observed larger pulses could be modelled to determine aggregate size (Figure 2.3). An observation of many pulses in close succession suggested that columnar aggregates (aligned by their dipole moments) were hydrodynamically separated as they moved through the pore. In a more quantitative study of $1.1 \mu \mathrm{m}$ SPBs [91], a bar magnet generated a magnetic field of up to $15 \mathrm{mT}$ at the tunable pore. Beads in the nearer half of the fluid cell were attracted away from the pore, reducing the pulse rate and increasing the FWHM duration as the magnet was moved closer. Size measurements suggested a lack of aggregation, and it was noted that the particles were at relatively low concentration, that aggregates could be sterically excluded from the pore, and that hydrodynamic forces in the pore constriction were strong enough to overcome dipole interactions.

Buchs et al. [214] measured the size of Se nanoparticles formed by conversion of dissolved Se into insoluble, high purity Se by aquatic organ- 
isms. The size distribution for these biogenic nanoparticles $(\sim 360 \mathrm{~nm})$ suggests a mean settling velocity of $2.93 \mathrm{~cm}$ per day. Such information could aid the efficient removal of Se nanoparticles from suspension, with positive environmental effects and potential benefits due to demand for Se in dietary supplements and industry, particularly photovoltaics. Yoon et al. [215] used TRPS to investigate novel amphiphilic Janus particles synthesized using electrohydrodynamic cojetting, with proposed applications related to self-assembly at interfaces. One population was found to have an average diameter of $2 \mu \mathrm{m}$, while the concentration of a second population (average diameter $300 \mathrm{~nm}$ determined by DLS) was found to be $5 \times 10^{7} \mathrm{~mL}^{-1}$. TRPS has been used to characterize cylindrical micelles which self-assemble from elastin-like polypeptides by genetically fusing an assembly domain to one end. The equivalent diameter (100-150 nm) was broadly in agreement with DLS [105]. Elsewhere [216], TRPS was an efficient high-throughput method for analysing raspberry-like particles consisting of $4.5 \mu \mathrm{m}$ polystyrene particles covered by various, smaller microgel spheres in $10 \mathrm{mM}$ formate buffer at $\mathrm{pH}$ 3.3. 


\subsection{Conclusion}

This Chapter has summarized the characteristics and roles of TRPS in recent applied research to provide context for this Thesis. Technical aspects such as pore specimens and stretching, and the qNano apparatus have been discussed. Concentration, size and charge analysis methods and measurement protocols were described. Comparative studies were outlined and the distinct characteristics and advantages of TRPS were highlighted. Applications in the areas of diagnostics and geonomics, EVs, nanomedicine, and biological samples among others were summarised and discussed. Currently, TRPS particle size, concentration and charge measurements are usually limited to calibration methods due to the complex geometry of the pores. Improved measurements of biological particles particularly in the category of EVs are limited by the lower size limit of the smallest available aperture $(\sim 50 \mathrm{~nm})$, and the complexity of biological solutions which can lead to pore blocking.

It can be seen that TRPS is increasing in popularity as a particle analysis technique (Figure 2.13), especially for bioparticles. The main particle characteristics measured are size and concentration and particles of interest 


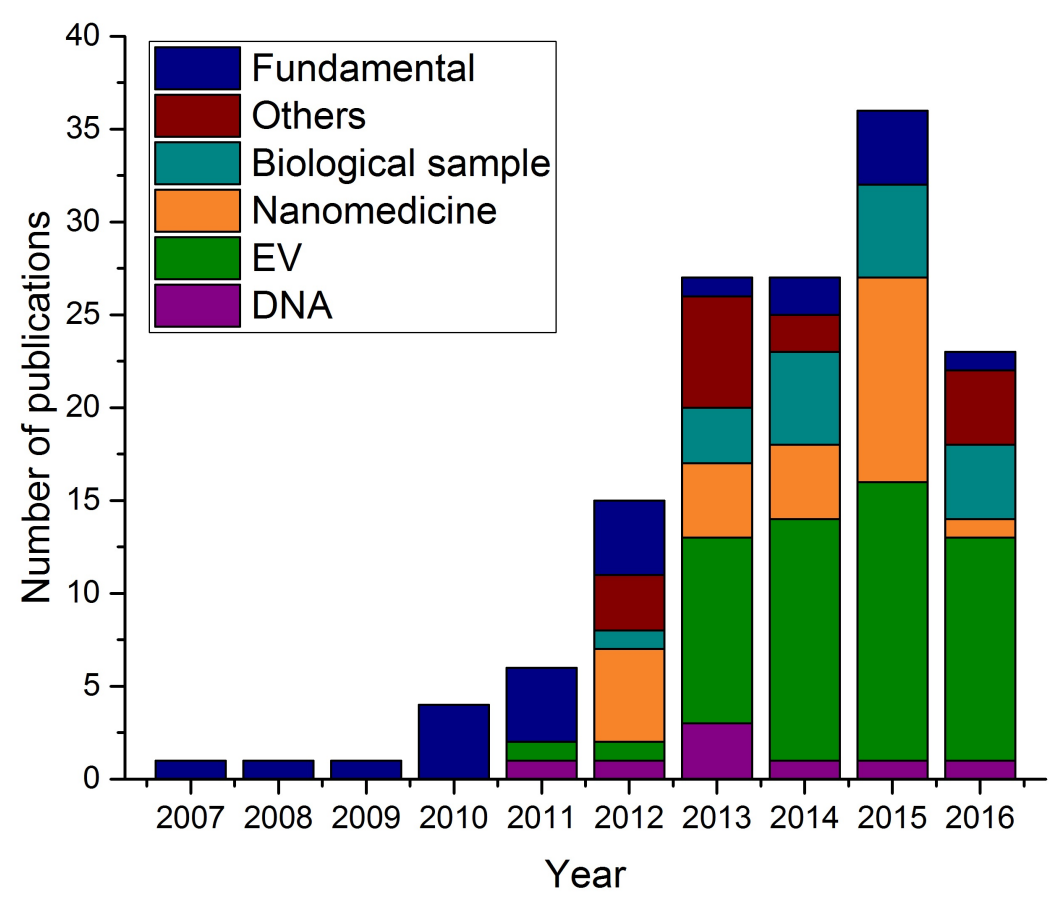

Figure 2.13: Published papers involving TRPS each year separated into categories DNA, EV, nanomedicine, biological samples, others and fundamental. Numbers were gathered from Izon Science's website on the 21st of September, 2016. 
are generally in the $100-400 \mathrm{~nm}$ size range. Particle charge is seldom measured but of late a handful of particle $\zeta$-potential studies have surfaced. However, uncertainties are often disregarded in these measurements and the measurement methods used have not been rigorously investigated or validated. For advancement and more widespread use of TRPS a better understanding of the technique is required especially in the area of particle surface charge. This understanding will benefit a large number of researchers in a range of scientific disciplines. 


\section{Chapter 3}

\section{Materials and Methods}

This Chapter details the materials, experimental set-ups and analytical procedures used to produce the results presented in Chapters 4, 5, 6 and 7. Section 3.1 reports the composition, characteristics and preparation procedures for all electrolytes, pores and particles used. Section 3.2 describes the experimental equipment and methods used including the qNano, VPM, pulse analysis methods (including software), streaming potential, Zetasizer, x-ray photoelectron spectroscopy (XPS), Fourier transform infrared spectroscopy (FTIR) and SEM. Section 3.3 details the pore coating methods. 


\subsection{Materials}

\subsubsection{Electrolytes}

Below, the composition of the electrolytes used are described. $\mathrm{pH}$ of electrolytes was confirmed by measurements using a calibrated PCS Testr35 $\mathrm{pH}$ meter. $\mathrm{pH}$ readings were taken after the $\mathrm{pH}$ meter held the same value in the solution for more than 10 seconds. $0.01-0.03 \% \mathrm{v} / \mathrm{v}$ Triton $\mathrm{X}-100$ (BDH Chemicals Ltd.) was added prior to TRPS experiments to increase dispersion stability unless otherwise stated. Prior to the addition of particles, electrolyte solutions were passed through a $0.45 \mu \mathrm{m}$ syringe filter (Minisart, Sigma-Aldrich).

\section{Standard Electrolyte Buffer}

Standard electrolyte buffer (SEB) consists of $0.1 \mathrm{M} \mathrm{KCl}$ (BDH Chemicals Ltd.), 15 mM 2-amino-2-hydroxymethyl-propane-1,3-diol (tris) (Sigma Aldrich) buffer, and $3 \mathrm{mM}$ ethylenediaminetetraacetic acid (EDTA) (Sigma Aldrich) in deionised water (Milli-Q, $18.2 \Omega \mathrm{cm}$ ). The solution $\mathrm{pH}$ was adjusted to 8.0 by the addition of $\mathrm{HCl}$. 
Phosphate Buffered Saline

PBS consists of one PBS tablet (Sigma Aldrich) was dissolved in $200 \mathrm{~mL}$ deionised water (Milli-Q, $18.2 \Omega \mathrm{cm}$ ) to create a $\mathrm{pH} 7.4$ solution containing $137 \mathrm{mM} \mathrm{NaCl}, 2.7 \mathrm{mM} \mathrm{KCl}, 10 \mathrm{mM} \mathrm{Na}_{2} \mathrm{PHO}_{4}$, and $1.8 \mathrm{mM} \mathrm{KH}_{2} \mathrm{PO}_{4}$. This standard PBS solution was diluted in some experiments to give the range of electrolyte concentrations.

\section{HEPES}

4-2-hydroxyethyl-1-piperazineethanesulfonic acid (HEPES) buffered solution consists of $100 \mathrm{mM} \mathrm{KCl}$ and $15 \mathrm{mM} \mathrm{HEPES} \mathrm{(Sigma} \mathrm{Aldrich)} \mathrm{in} \mathrm{deionised}$ water (Milli-Q, $18.2 \Omega \mathrm{cm}$ ). The solution $\mathrm{pH}$ was adjusted to 7.0 by the addition of $\mathrm{NaOH}$.

$\mathrm{KCl}$

$\mathrm{KCl}$ solutions consist of $\mathrm{KCl}$ (concentrations stated in experimental chapters) in deionised water (Milli-Q, $18.2 \Omega \mathrm{cm}$ ). The solution $\mathrm{pH}$ was sometimes adjusted for specific experiments using $\mathrm{HCl}$ and $\mathrm{NaOH}$. 


\begin{tabular}{|c|c|c|c|c|c|c|c|c|}
\hline Pore ID & NP rating & $P\left(\mathrm{~cm} \mathrm{H}_{2} \mathrm{O}\right)$ & $V_{0}(\mathrm{~V})$ & Stretch $(\mathrm{mm})$ & Electrolyte & $a \operatorname{SEM}(\mathrm{nm})$ & $b \operatorname{SEM}(\mathrm{nm})$ & Experiments \\
\hline A20403 & NP150 & 0 & $0.1-0.6$ & 45 & SEB & - & - & Section 4.2.1 pore (i) \\
\hline A20407 & NP150 & 0 & $0.1-0.6$ & 45 & SEB & 2917 (ripped) & 10079 & Section 4.2.1 pore (ii) \\
\hline A21820 & NP150 & 0 & $0.2-0.7$ & 47 & SEB & 1666 & 35959 & Section 4.2.1 pore (iv) \\
\hline A21830 & NP150 & 0 & $0.2-0.7$ & 47 & SEB & - & - & Section 4.2.1 pore $(\mathrm{v})$ \\
\hline A12673 & NP200 & 0 & $0.1-0.7$ & 45 & SEB & - & - & Section 4.2.1 pore (iii) \\
\hline A28818 & NP150 & 0 & 0.44 & $50-50.5$ & PBS & 645 & 24664 & Section 4.2.3 Figure 4.11 \\
\hline A28818 & NP150 & 0 & 0.44 & 50 & HEPES & 645 & 24664 & Section 4.2.3 Figure 4.11 \\
\hline A28818 & NP150 & 0 & $0.44-0.50$ & $48-50$ & SEB & 645 & 24664 & Section 4.2.3 Figure 4.11 \\
\hline A31629 & NP150 & -0.5 & $0.16-0.48$ & $45.5-48$ & PBS (100-200\%) & 624 & 57570 & Section 4.2.3 Figure 4.12 \\
\hline A28824 & NP150 & -0.5 & $0.60-1.08$ & $46.5-47$ & PBS $(90 \%$ and $60 \%)$ & - & - & Section 4.2.3 Figure 4.12 \\
\hline A28952 & NP150 & -0.5 & $0.54-0.66$ & 46.9-52 & PBS $(80 \%, 70 \%, 50 \%)$ & - & - & Section 4.2 .3 Figure 4.12 \\
\hline А 32029 & NP200 & - & - & $45-52$ & PBS $(10 \%$ and $100 \%)$ & 813 & 27315 & Section 5.3.1 Figure 5.3 \\
\hline A12353 & NP400 & - & - & $45-52$ & PBS $(10 \%$ and $100 \%)$ & 397 & 45629 & Section 5.3.1 Figure 5.3 \\
\hline A22300 & NP800 & - & - & $45-52$ & PBS ( $10 \%$ and $100 \%)$ & 1212 & 46912 & Section5.3.1 Figure 5.3 \\
\hline A40358 & NP150 & -0.5 & $0.34-0.38$ & $50-52$ & PBS & - & - & Section 5.3.1 Figure 5.4 \\
\hline A40357 & NP150 & -0.5 & $0.78-1.20$ & $45.5-47.5$ & PBS (50\%) & 194 & 32739 & Section 5.3.2 \\
\hline A40357 & NP150 & -0.5 & $0.44-0.68$ & $44.6-48$ & PBS (100\%) & 194 & 32739 & Section 5.3.2 \\
\hline A40357 & NP150 & -0.5 & $0.2-0.3$ & $47-50$ & PBS (200\%) & 194 & 32739 & Section 5.3.2 \\
\hline A40339 & NP150 & - & - & 52 & $\mathrm{KCl}(\mathrm{pH} 2-12)$ & - & - & Section 5.3.3 Figure 5.7 \\
\hline A40338 & NP150 & -0.5 & 0.62 & 47 & $\mathrm{KCl}(\mathrm{pH} 4)$ & - & 31156 & Section 5.3.3 Figure 5.8 \\
\hline A40338 & NP150 & -0.5 & $0.60-1.06$ & $45.2-50$ & $\mathrm{KCl}(\mathrm{pH} 6)$ & - & 31156 & Section 5.3.3 Figure 5.8 \\
\hline A40338 & NP150 & -0.5 & $0.60-1.06$ & $45.2-51.6$ & $\mathrm{KCl}(\mathrm{pH} 8)$ & - & 31156 & Section 5.3.3 Figure 5.8 \\
\hline A40338 & NP150 & -0.5 & $1.06-1.32$ & $45.2-45.5$ & $\mathrm{KCl}(\mathrm{pH} 10)$ & - & 31156 & Section 5.3.3 Figure 5.8 \\
\hline A41972 & NP150 & 0 & $-0.6-0.7$ & 46 & PBS & 482 & 32686 & Section 5.4.1 Figure 5.15 \\
\hline A41984 & NP150 & 0 & $-0.8-0.8$ & 45 & PBS & - & - & Section 5.4.1 Figure 5.15 \\
\hline
\end{tabular}




\begin{tabular}{|c|c|c|c|c|c|c|c|c|}
\hline A28972 & NP100 & 0 & $-1.6-1.6$ & 45 & PBS & - & - & Section 5.4.1 Figure 5.16 \\
\hline A41986 & NP150 & 0 & $-1.6-1.6$ & 45 & PBS & - & - & Section 5.4.3 \\
\hline NHH23 & NP2000 & - & - & 52 & PBS and 10 mM KCl & 1208 & 30838 & Section 5.4.2 \\
\hline NHH40 & NP2000 & - & - & 52 & PBS and 10 mM KCl & - & - & Section 5.4.2 \\
\hline NHH31 & NP400 & - & - & 52 & PBS and 10 mM KCl & - & - & Section 5.4.2 \\
\hline NHH32 & NP400 & - & - & 52 & PBS and 10 mM KCl & - & - & Section 5.4.2 \\
\hline NHH14 & NP100 & - & - & 52 & PBS and 10 mM KCl & - & - & Section 5.4.2 \\
\hline NHH17 & NP100 & - & - & 52 & PBS and 10 mM KCl & - & - & Section 5.4.2 \\
\hline A21032 & NP150 & 0 & -1.6 & 47 & PBS (10\%) & 152 & - & Section 6.3.2 \\
\hline A23802 & NP150 & 0 & $-0.15-1.6$ & 49 & PBS (5-100\%) & 176 & - & Section 6.3.3. 6.3.4 and 6.3.6 \\
\hline A25135 & NP150 & $-10-10$ & -1.6 & 49.5 & PBS (10\%) & 600 & - & Section 6.3.1 Figure 6.2 \\
\hline A27082 & NP150 & $0--14$ & 1.6 & 52 & PBS (10\%) & 158 & 44022 & Section 6.3.1 Figure 6.3 \\
\hline A25136 & NP150 & 0 & -0.70 & $46-50$ & PBS (10\%) & 576 & - & Section 6.3.5 \\
\hline A33701 & NP150 & 0 & 0.32 & $45-52$ & SEB & 987 & 23250 & Chapter 7 pore 1 Stretch \\
\hline A33701 & NP150 & $0-8$ & 0.56 & 48 & SEB & 987 & 23250 & Chapter 7 pore 1 Pressure \\
\hline A33701 & NP150 & 0 & $0.20-0.60$ & 48 & SEB & 987 & 23250 & Chapter 7 pore 1 Voltage \\
\hline A33700 & NP150 & 0 & 0.32 & $45-52$ & SEB & - & - & Chapter 7 pore 2 Stretch \\
\hline A33700 & NP150 & $0-8$ & 0.56 & 48 & SEB & - & - & Chapter 7 pore 2 Pressure \\
\hline A21599 & NP400 & 0 & 0.22 & $45-52$ & SEB & - & - & Chapter 7 pore 3 \\
\hline A11134 & NP200 & $0-10$ & $0.28-1.02$ & $44-50$ & SEB & - & - & Chapter 7 pore 4 \\
\hline
\end{tabular}

Table 3.2: Summary of pores used. SEM images of pores can be found in Appendix B. 


\subsubsection{Pores}

Pore specimens (see Table 3.3) were obtained from Izon Science (Christchurch, NZ). Pore ratings were provided by Izon Science indicating the particle size range each pore is suitable for. This pore rating comes in the form 'NP' followed by the optimal particle diameter for that pore. All pores used are summarised in Table 3.2. The pressure, voltage, stretch and electrolyte conditions for each experiment are listed. Specific values for pore geometries are included where possible from SEM imaging. Pores were placed on the qNano in the normal orientation (small opening at the top, large at the bottom) unless otherwise stated. Stretch is defined as the distance $(X)$ measured (with Vernier callipers) between the outer edges of the teeth on which the membrane is set at the time of measurements. The macroscopic strain is defined as $\alpha=\left(X-X_{0}\right) / X_{0}$, where unstretched jaw separation $X_{0}$ is taken to be $42 \mathrm{~mm}$. Specimens were pre-stretched to a minimum of $X=49 \mathrm{~mm}$ for 5 minutes prior to experimental use. 


\begin{tabular}{|c|c|c|c|c|c|c|}
\hline Label & Supplier ID & Manufacturer/Supplier & Nominal diameter $(\mathrm{nm})$ & TRPS diameter $(\mathrm{nm})$ & Surface charge density $(\mu \mathrm{eq} / \mathrm{g})$ & Experiments \\
\hline A & 3393 & Bangs Laboratories & 220 & $213 \pm 2.5$ & 47 & Chapter 4,6 \\
\hline B & $6481 /$ CPC200 & Bangs Laboratories & 200 & $213 \pm 2.5$ & 86 & Chapter $4,5,6,7$ \\
\hline C & 9522 & Bangs Laboratories & 200 & $188 \pm 2.5$ & 120.8 & Chapter 4,6 \\
\hline D & 6569 & Bangs Laboratories & 220 & $173 \pm 2.5$ & 244.4 & Chapter 4,6 \\
\hline E & CPN200 & Izon Science & 200 & $183 \pm 3.1$ & - & Chapter 4,5 \\
\hline F & 11777 & Bangs Laboratories & 200 & $200 \pm 3.5$ & - & Chapter 5 \\
\hline G & 9522 & Bangs Laboratories & 200 & $179 \pm 2.8$ & 120.8 & Chapter 5 \\
\hline H & 10164 & Bangs Laboratories & 200 & $181 \pm 2.6$ & 159 & Chapter 5 \\
\hline I & 6570 & Bangs Laboratories & 250 & $183 \pm 2.9$ & 159.2 & Chapter 5 \\
\hline J & 6569 & Bangs Laboratories & 220 & $164 \pm 2.6$ & 244.4 & Chapter 5 \\
\hline K & 6555 & Bangs Laboratories & 260 & $192 \pm 3.1$ & 638.2 & Chapter 5 \\
\hline L & CPC400 & Polysciences/Izon Science & 350 & - & - & Chapter 7 \\
\hline M & CPC200 & Thermo-Fisher/Izon Science & 220 & - & - & Chapter 7 \\
\hline
\end{tabular}

क

Table 3.3: Summary of particles used. 


\subsubsection{Particles}

Prior to TRPS experiments particles were added to the desired electrolyte at a concentration of $\sim 10^{10} \mathrm{~mL}^{-1}$ and dispersed by vortexing for $10 \mathrm{sec}-$ onds and sonicated at high power for 10 minutes. Particle sets used in TRPS experiments are listed in Table 3.3. The main suppliers of particles were Izon Science (Christchurch, NZ), and Bangs Laboratories (Fishers, IN, USA). Bangs Laboratories provide a surface charge density value for their carboxyl functionalized particles. This value has the units micro equivalents of $\mathrm{COOH}$ groups per gram of particles. A micro equivalent is defined as a mole $\left(6.022 \times 10^{23}\right)$ of charged groups [217]. The surface charge density can be used as an indicator of particle charge.

Additionally, 6 sets of carboxylated polystyrene calibration particles (CPCs) and 6 sets of non-carboxylated polystyrene calibration particles (CPNs) were investigated using DLS in Chapter 4. These particle sets were supplied by Izon Science. Further details of these particles sets can be found in Table 4.4 . 


\subsection{Experimental Equipment}

\subsection{1 qNano}

TRPS was performed using the qNano (pictured and described in Section 2.1.2) obtained from Izon Science. Typically $80 \mu \mathrm{L}$ of electrolyte was added to the lower fluid cell and $40 \mu \mathrm{L}$ of particles suspended in electrolyte was added to the upper fluid cell. In experiments when particles were moving in both directions through the pore, $80 \mu \mathrm{L}$ of particles suspended in electrolyte would be added to the lower fluid cell.

\subsubsection{VPM}

The inherent pressure head is kept consistent by using consistent volumes of liquid (40 $\mu \mathrm{L}$ and $80 \mu \mathrm{L}$, respectively) in the upper and lower fluid cells. The inherent pressure head has been measured as $0.47 \mathrm{~cm} \mathrm{H}_{2} \mathrm{O}$ [47]. Pressure can be applied in addition to this using the VPM which connects to the upper fluid cell. Once the valve is shut, between $0.1-20 \mathrm{~cm} \mathrm{H}_{2} \mathrm{O}$ can be applied in $0.1 \mathrm{~cm} \mathrm{H}_{2} \mathrm{O}$ increments. 


\subsubsection{Pulse Analysis}

The Izon Control Suite (ICS) software was used to collect TRPS data. Current trace information is recorded at $50 \mathrm{kHz}$ as a binary Izon raw blockade data '.irbd' file with columns recording time (s), current (nA), bias (V) and applied stretch $(\mathrm{mm})$ respectively. Once processed this .irbd file is replaced with a .idfs file from which samples can be analysed within ICS. To analyse TRPS data outside of ICS the .irbd file or an equivalent .csv file are required. This can be generated in post processing by exporting the raw data of the idfs file.

The trace analysis run by ICS consists of generating a running average of the current (baseline current) across a time window (typically $100 \mathrm{~ms}$ ). The trace is then scanned for multiple consecutive data points that deviate from the baseline current by more than a set amount (0.05\%). Event durations, defined as the time between the first data point deviating from the baseline by $0.05 \%$ and returning to within $0.05 \%$, also have a minimum threshold which is set at $0.2 \mathrm{~ms}$. There is a maximum data collection time period of 10 minutes at which point a recording will be terminated.

Other software methods used to examine TRPS data include the python 
.irbd process program for biphasic pulses [99] (Chapter 6), Microsoft Excel combined with OriginPro 2016 for analysing particle size distribution broadening (Chapter 7) and Clampfit 10.1 for looking at entire traces. The python irbd process program was written by Eldridge [99]. It converts a .irbd file to a .dat file and from that produces a .csv file output of the times, magnitudes and durations of conductive and resistive pulses. The time window of the average baseline and event magnitude threshold must be specified by the user. These were typically set at $100 \mathrm{~ms}$ and $0.05 \%$ respectively.

Particle size distribution histograms analysed for distribution broadening (Chapter 7) were produced by exporting the .csv file containing individual blockade magnitudes (units $=\mathrm{nA}$ ) from ICS and converting these into histograms (bin size $=0.025)$ of the normalised cubed root magnitude. Data were then transferred to OriginPro. OriginPro plots were fitted with single and double Gaussian peaks to analyse distribution broadening. When double peaks were fitted the centre of these peaks was estimated prior to fitting. The coefficient of variance $(\mathrm{CV})$ of whole distributions was determined by dividing the standard deviation of all blockades by the average of all blockades. CVs of double peaks within a distribu- 
tion were determined using OriginPro's average and standard deviation outputs.

Clampfit is a useful piece of software for viewing and exporting current traces for an entire measurement. In particular this was used when looking at oscillations (Section 5.5). ICS file raw data are exported as a .csv file. This is imported into Clampfit where the entire current trace can be viewed. Observing the entire current trace is not a feature available in ICS.

\subsubsection{Zetasizer}

Comparative and calibration size and $\zeta$-potential measurements were made using DLS with the Zetasizer Nano (Malvern). Universal dip cells (UDCs) (ZEN1002, Malvern) in combination with disposable polystyrene cells were used for DLS $\zeta$-potential measurements after initial comparison measurements with disposable folded capillary cells (DFCCs) (DTS1070, Malvern). DLS particle size measurements were taken in disposable polystyrene cells. Data analysis used the Smoluchowski model for aqueous solutions, and the analysis model Auto Mode, with refractive indices for the dispersant and particles of 1.333 and 1.59 (respectively) and an absorption of 0.01 for 
the particles. The dispersant was the same solution used in TRPS experiments, with relevant parameters set at $25^{\circ} \mathrm{C}$ using the instrument software. For DLS particle size measurements 3-10 samples were prepared and analysed for each particle set studied, with 20-100 runs per sample. To enable like-for-like comparisons of particle size distribution histograms with other data sets, one log-scale population plot representative of all measurements was chosen, and data were extracted using Engauge Digitizer (sourceforge.net). For DLS particle $\zeta$-potential measurements 3-10 samples were prepared and analysed for each particle set studied, with 5 runs per sample.

\subsubsection{XPS}

XPS data for TPU and dopamine coated TPU surfaces were obtained using a Kratos Axis Ultra DLD spectrometer equipped with a monochromatic Aluminium K $\alpha$ source $(1486.6 \mathrm{eV})$. Elemental survey scans were run five times on all samples with pass energy of $160 \mathrm{eV}$, and step size of $1 \mathrm{eV}$. Chemical state scans of the sulfur, nitrogen, oxygen and carbon regions were conducted with pass energy of $20 \mathrm{eV}$, and step size of $0.1 \mathrm{eV}$. Quantification of the survey scans and fitting of the chemical state scans were 
performed using the CasaXPS package [218]. The binding energy scale was corrected for the neutralizer shift using the $C$ 1s signal from saturated hydrocarbon at $285.0 \mathrm{eV}$ as an internal standard.

\subsubsection{FTIR}

IR data for TPU and dopamine coated TPU surfaces were obtained using a Perkin Elmer Spectrum 1 FTIR instrument fitted with a universal attenuated total reflectance (ATR) sample accessory. The ZnSe prism was cleaned prior to each experiment using Kimwipes (Sigma Aldrich). All data collections used 4 scans. A background spectrum was collected from the cleaned prism between experiments.

\subsubsection{SEM}

\section{Particles}

SEM images were acquired under high vacuum using the FEI Nova NanoSEM 450 microscope (at Callaghan Innovation, Lower Hutt) operated at $5 \mathrm{kV}$ (Figure 3.1). Images were analysed using ImageJ software [219] to obtain 


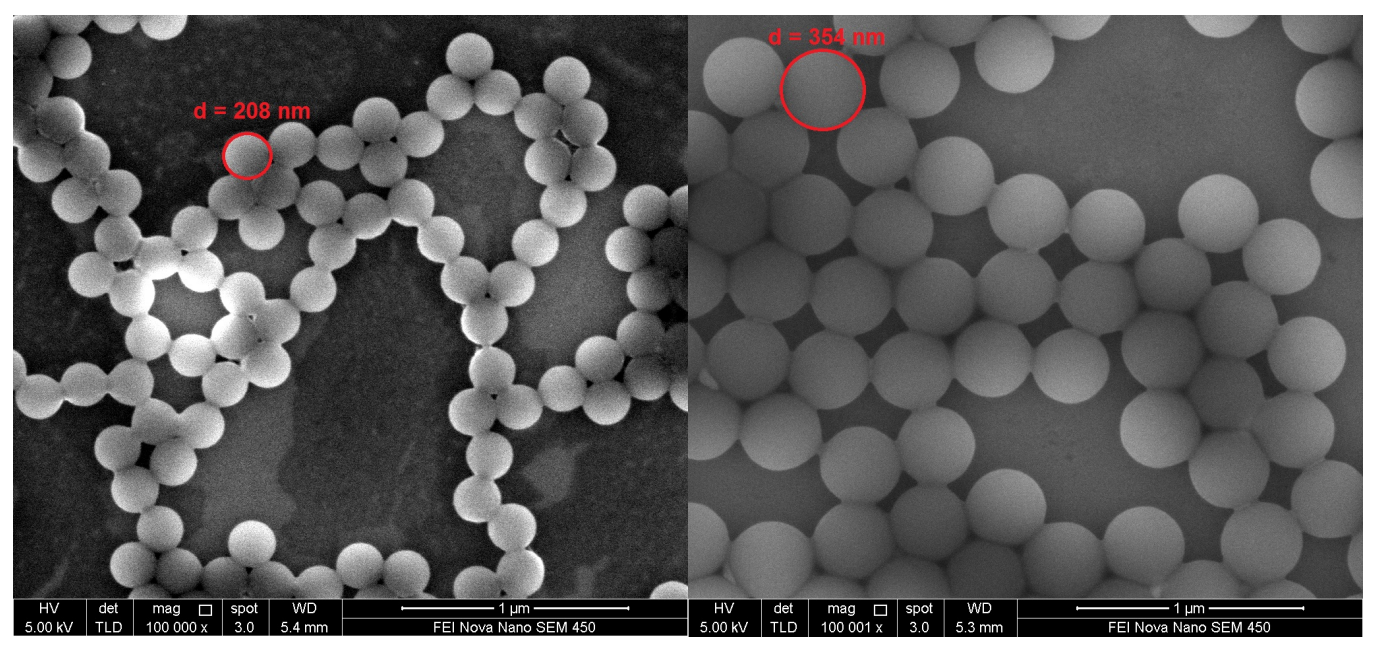

Figure 3.1: Example of SEM images used to size particle sets B (left) and $\mathrm{M}$ (right).

the diameter of 500 particles per particle set. ImageJ was calibrated using the scale bar. The outline of the particles was then drawn using the circle tool and the effective diameter measured using the software (Figure 3.1).

\section{Pores}

Pores were imaged, after being used for TRPS measurements, using a Quanta SEM (at Callaghan Innovation, Lower Hutt) (Figure 3.2 and Appendix B). Pores were mounted on a custom-fabricated stretching stage using the eyelets in the sample, and carbon tape was applied around the pore opening to minimise charging effects. The location of the large pore opening was approximately determined using optical microscopy prior 


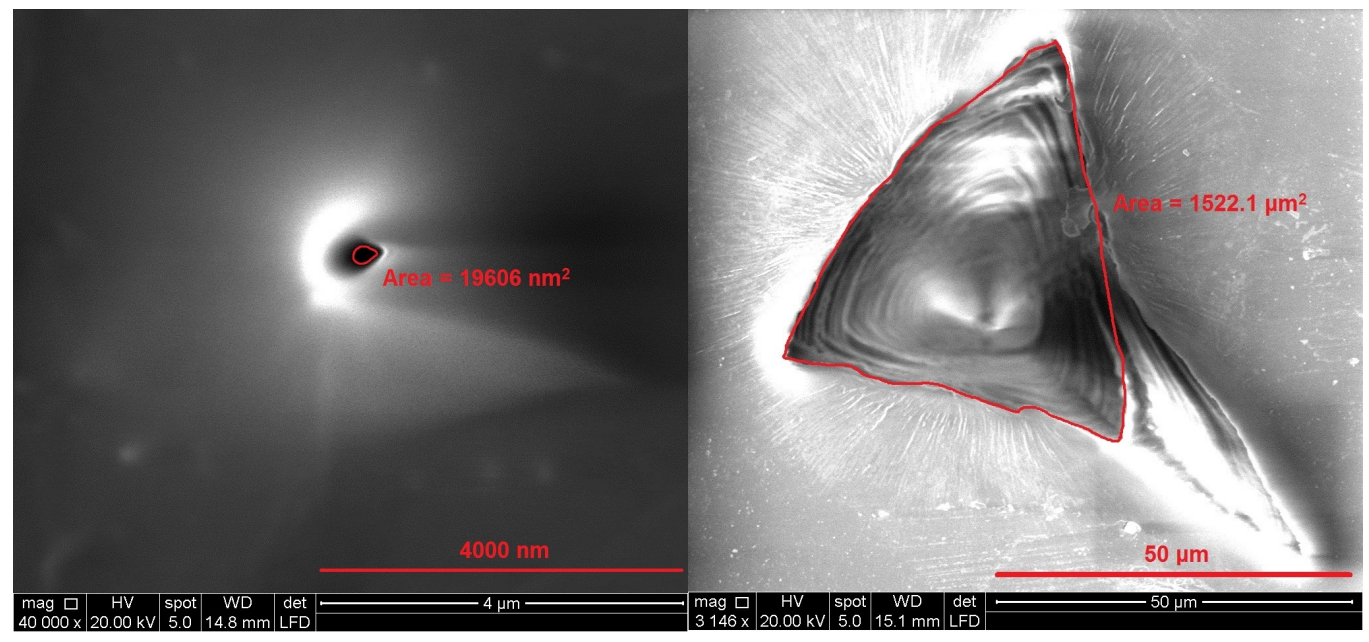

Figure 3.2: Example of SEM of the small (left) and large (right) pore openings of A27082.

to SEM, and the samples marked with a needle to enable location of the large and small pore openings under SEM. The SEM stretching stage teeth used were at $X=45 \mathrm{~mm}$. Images were analysed using ImageJ software to obtain the diameters of the small and large pore openings. ImageJ was calibrated using the scale bar. The outline of the pore was then traced with the free draw tool and the effective diameter was then measured using the software (Figure 3.2). 


\subsection{Pore Coating Methods}

\subsubsection{Dopamine and 'Fe + Dopamine'}

$80 \mu \mathrm{L}$ of $2 \mathrm{mg} / \mathrm{mL}$ dopamine hydrochloride (Sigma Aldrich) in $10 \mathrm{mM}$ tris buffer at $\mathrm{pH} 8.5$ was placed in the lower fluid cell under a new pore stretched to $X=52 \mathrm{~mm}$. A vacuum of $20 \mathrm{~cm} \mathrm{H}_{2} \mathrm{O}$ was applied to draw solution through the pore. After 15 hours the pore was washed with deionised water and dried with compressed air before measurements were taken. In the case of 'Fe + Dopamine' $2 \mathrm{mg} / \mathrm{mL}$ iron(II) chloride tetrahydrate (Sigma Aldrich) was added to the coating solution.

\subsubsection{PEI/PAA}

$80 \mu \mathrm{L}$ of $5 \%$ wt in $\mathrm{H}_{2} \mathrm{O}$ polymer solution, either polyethylenimine (PEI) (Sigma Aldrich) or polyacrylic acid (PAA) (Sigma Aldrich), was placed in the lower fluid cell under a new pore stretched to $52 \mathrm{~mm}$ for 2 hours. The pore was washed with deionised water and dried with compressed air before measurements were taken. The pore was then incubated with a second polymer layer for 2 hours and again washed with deionised water 
and dried with compressed air before measurements were taken. This process was repeated until the desired number of layers was obtained. 


\section{Chapter 4}

\section{Initial $\zeta$-potential Measurements}

In this Chapter, $\zeta$-potential measurements made using a number of methods developed for TRPS are described. These methods (Methods I-IV) are iterations of each other in an attempt to refine and improve the technique. In this Chapter, these methods are initially introduced and the related charge measurement uncertainties are assessed. Then, results obtained using Methods I and II are compared. Finally, Methods III and IV are developed and applied to particles in a range of electrolyte conditions. Uncertainties are calculated, analysed and compared for Methods II and IV. DLS measurements of $\zeta$-potential were made alongside TRPS 
measurements for comparison. In Chapter 5, Method IV is improved on and further $\zeta$-potential measurements are made.

$\zeta$-potential is an important property of colloidal dispersions. It gives information about particle composition, dispersion stability and the nature of the particle-liquid interface. Conventional $\zeta$-potential measurement techniques (e.g. DLS) rely on ensemble measurements which are inherently sensitive to sub-populations of larger particles in a sample. A number of TRPS $\zeta$-potential measurement methods have been reported $[46,47,111,112]$. However, there are challenges associated with each of these methods and further work is needed.

Vogel et al. [47] first used TRPS to demonstrate $\zeta$-potential measurement using resistive pulse rates. Kozak et al. [82] built position-in-time (velocity) profiles for individual particles based on experimental data. With the total pressure applied across the pore still precisely controlled, the $\zeta$ potential of individual particles could be calculated [46]. Blundell et al. [112] were the first to demonstrate Method II (defined below) using DNA coated particles. Sikora et al. [114] and Blundell et al. [220] used Method II to deduce the $\zeta$-potential of silica and polystyrene nanoparticles respectively before and after the absorption of proteins. 
Here, the use of single particle $\zeta$-potential analysis methods is investigated in practice. A method similar to Kozak et al.'s technique, in which the entire resistive pulse data trace is analysed (Method I), is compared with a simplified method examining discrete points along the resistive pulse (Method II). The effects of voltage and specimen fabrication on $\zeta$ potential measurements are explored using both methods. Further iterations, Method III and Method IV, are applied to particles in different electrolyte conditions including different salts and different molarities.

\subsection{Theory and Analysis}

$\zeta$-potential is a measurement of surface charge. When a charged solid surface (such as a particle or a pore) comes into contact with an ionic solution the surface attracts counter-ions, repels co-ions and establishes a distribution of ions that decays away from the surface. This distribution of charges, usually modelled as a Boltzmann distribution, is known as the EDL. In the Gouy-Chapman-Stern model [221] the EDL is divided into two regions dependent on the kinetic properties of the ions occupying them (Figure 4.1). The inner layer, often called the Stern layer, is a compact re- 


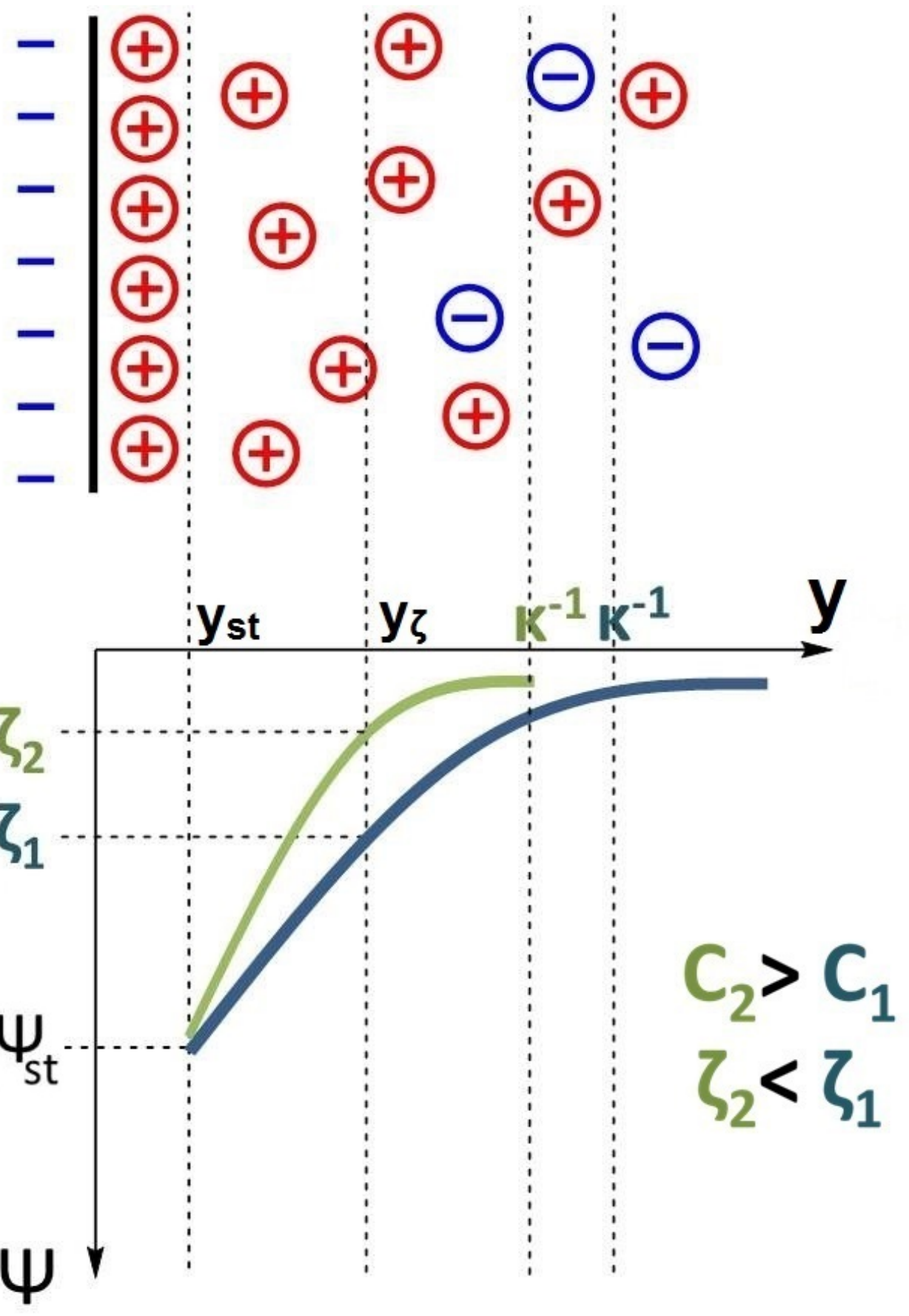

Figure 4.1: EDL diagram showing the layers that make up the doublelayer, adapted from [90,99]. The surface is illustrated with a negative surface charge. Compression of the double layer is shown in green when the surface is exposed to a higher concentration electrolyte. $\Psi$ is the potential, $\zeta$ is the $\zeta$-potential, and $\kappa^{-1}$ is the Debye length. The EDL of the higher electrolyte concentration $C_{2}$ is shown in green, the lower electrolyte concentration $C_{1}$ is shown in blue. 
gion of immobilised ions very near the surface. The distribution of ions in the inner layer is determined by geometrical restrictions and short range interactions between ions and the surface. The distance this layer extends from the surface (distance $y_{s t}$ at which the potential is $\Psi_{s t}$ ) is therefore determined by the size of the counter ions. The outer layer is a diffuse layer containing a Boltzmann distribution of ions described by

$$
\Psi=\Psi_{0} e^{-\kappa y}
$$

where $\Psi$ is the electrostatic potential at $y$ distance from the surface, $\Psi_{0}$ is the electrostatic potential at the surface $(y=0)$ and $\kappa$ is the inverse of the length of the EDL, often called the Debye length. The Debye length in a 1:1 electrolyte in water at $25^{\circ} \mathrm{C}$ is given by

$$
\kappa^{-1}=\frac{0.304}{\sqrt{I S}}
$$

where $I S$ is the ionic strength in $\mathrm{mol} \mathrm{L}^{-1}$ and $\kappa^{-1}$ is in $\mathrm{nm}$. Increasing the ionic strength causes a decrease in $\kappa^{-1}$ and as a result the potential falls off more quickly with $y$. This is referred to as compression of the EDL (Figure 
4.1).

At some distance into the diffuse layer the viscous forces in the bulk fluid balance the electrostatic forces immobilizing ions to the surface. This distance $\left(y_{\zeta}\right)$ is called the shear plane. Ions inside this plane are immobilised and will stay with the surface as either it moves (particle) or the fluid around it is moved (pore). Inside the shear plane the surface and surrounding ions act as a single entity. The potential at the shear plane is the $\zeta$-potential. $\zeta$-potential is therefore measured when examining electrophoretic mobility, electroosmosis or streaming potential as these measurements examine motion beyond the shear plane. $\Psi_{0}$, the actual electrostatic potential of the surface, is experimentally problematic to measure due to the shielding of ions inside the shear plane. The $\zeta$-potential can be expressed as a function of the surface charge density in strong electrolyte conditions $[222,223]$

$$
\sigma \approx \varepsilon \kappa \zeta
$$

where $\sigma$ is the surface charge density. $\zeta$-potential is therefore also related to the concentration and type of ions present in the solution environment 
through $\kappa$ (Equation 4.2).

In TRPS the particle $\zeta$-potential is extracted from particle velocity. The Nernst-Planck equation (Equation 2.3 [43]) describes particle velocity for the relevant particle transport mechanisms, namely electrophoresis, electroosmosis and pressure-driven flow. Diffusion and dielectrophoresis are assumed negligible because of the level of pressure and the low voltages applied, respectively [42].

$\zeta_{\text {particle }}$ is calculated from the electrophoretic mobility which is defined as the particle velocity in an electric field;

$$
v^{e p h}=\mu_{e p h} E
$$

where $v^{e p h}$ is the electrophoretic velocity, $\mu_{e p h}$ is the electrophoretic mobility and $E$ is the electric field strength. The relationship between electrophoretic mobility and $\zeta$-potential based on the Smoluchowski approximation is

$$
\mu_{e p h}=\frac{\varepsilon \zeta_{\text {particle }}}{\eta}
$$


The Smoluchowski or thin EDL approximation is applied for all TRPS measurements. This is suitable for measurements of $\zeta$-potential where the Debye length is much smaller than particle size.

$\zeta_{\text {pore }}$ is also important as it contributes to the total particle velocity from which $\zeta_{\text {particle }}$ is extracted. Electroosmosis occurs due to the movement of ions in the pore wall double layer. The electroosmotic mobility is the opposite of Equation 4.5;

$$
\mu_{e o}=-\frac{\varepsilon \zeta_{\text {pore }}}{\eta}
$$

In order to calculate $\zeta_{\text {particle }}$ it is necessary to understand this electroosmotic component and also any pressure that may be contributing to the total velocity of the particle.

$\zeta$-potential measurement using RPS and TRPS has undergone an evolution of techniques. $\zeta$-potential measurement methods developed for TRPS include the variable pressure method [47], the full blockade mapping method [46], a modified version of the latter using calibration particles [48] (here Method I), and finally the multi-point blockade methods 
using calibration particles $[48,112]$ (here Methods II-IV) which have been extended in this research. These methods are described below followed by an assessment of uncertainty for $\zeta$-potential measurements.

\subsubsection{Variable Pressure Method}

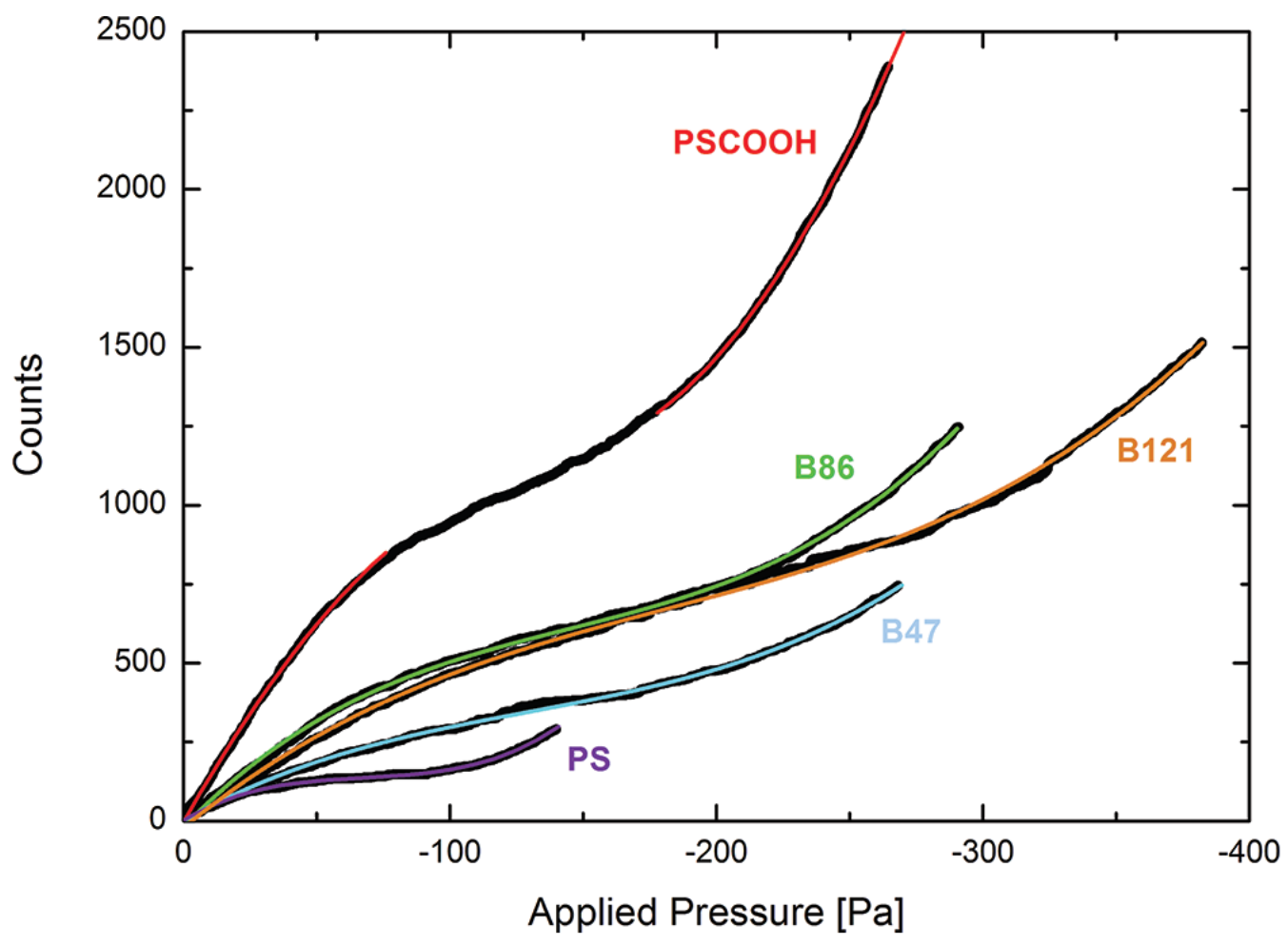

Figure 4.2: S-curves for a range of polystyrene particles of different charges. These data were used to measure particle $\zeta$-potentials using the variable pressure method. Adapted from [47].

The variable pressure method was the first method used to obtain $\zeta$ - 
potential values using TRPS [47]. Using this method pressure is varied either continuously or in discrete steps, and the pressure $\left(P_{0}\right)$ at which the forces acting on a particle are balanced and $\mathbf{J}=0$ is identified using either the greatest value of FWHM duration or the minimum pulse rate [111]. In the latter case, a plot of pressure against particle count gives an "S-curve" with a plateau representing the pressure at which the particle rate is minimal (Figure 4.2). When $\mathbf{J}=0$, Equation 2.3 can be re-written as

$$
\zeta_{\text {particle }}=\frac{\eta \mathbf{Q}}{\varepsilon \mathbf{E} A}-\zeta_{\text {pore }}
$$

where $\mathbf{Q}$ (Equation 2.5) and $\mathbf{E}$ [47] can be calculated given a known $P, V$ and pore geometry. These calculations assume that particle transport is parallel to the pore axis and pore diameter varies slowly with pore length.

It can therefore be seen that if the geometry of the pore $(a, b, l$ and $A)$ and $\zeta_{\text {pore }}$ are known, $\zeta_{\text {particle }}$ can be calculated. This technique has been used to measure the $\zeta$-potential of oil-in-water emulsions [14] and been investigated in detail by Eldridge et al. [99, 111]. 


\subsubsection{Velocity Profile Method and Method I}

Kozak et al. [46] simultaneously measured the $\zeta$-potential and size of particles using their velocity profile method. With a known pore geometry, resistive pulse current with time data could be converted to position-intime or velocity data. Using Equation 2.3 this velocity data can be assigned to the contributing forces: electrophoretic, electroosmotic and pressuredriven flow (Figure 4.3). With known $\zeta_{\text {pore }}$ and $P$, the electroosmotic and pressure driven flow components can be subtracted from the total velocity to extract the electrophoretic component. From this the $\zeta$-potential can be calculated.

Due to uncertainity in the pore geometry, Method I was developed. This method uses calibration particles of known diameter and $\zeta$-potential to determine pore geometry. Pores are assumed to be truncated linear cones, with openings of diameter $a$ and $b$ at the surfaces of a membrane of thickness $l$. Each of these dimensions changes with stretch. $l$ has been precharacterized as a function of $X$ for Izon Science's TPU membranes [82]. a is calculated by measuring $\Delta R$, the maximum resistance change from the baseline value $R_{0}$, for resistive pulses generated by a particle set of known 


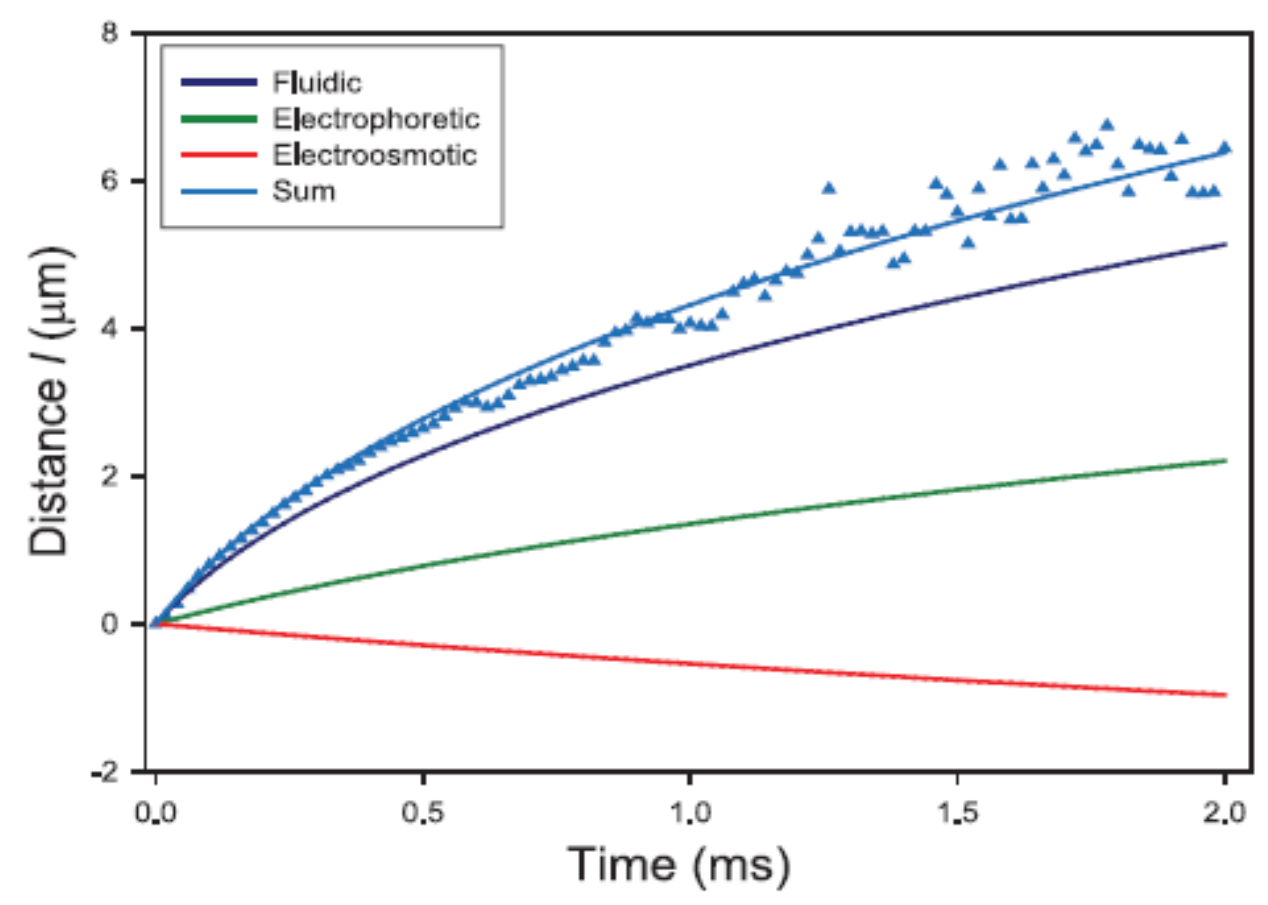

Figure 4.3: Position-in-time plot for a single blockade showing how the electrophoretic, electroosmotic and pressure-driven flow (fluidic) components can be separated out. Adapted from [46]. 
diameter $d$. Assuming that the pore diameter near the particle is $a$ when this maximum occurs, then

$$
a=\left(\frac{4 \rho d^{3}}{\pi \Delta R}\right)^{1 / 4} .
$$

$b$ is calculated using calibration particles with an assigned $\zeta$-potential. The end result of this process is that for each pore specimen used, the geometry $(a, b$ and $l)$ is defined at any given applied stretch. With the pore geometry established, particle position-in-time is calculated using data after the time of the maximum change in current for each experimental resistive pulse, corresponding to when the particle is entirely within the pore, in order to avoid end effects. With the geometry known and $\zeta_{\text {pore }}$ determined for the pore material $[14,46,47,99,111], \zeta_{\text {particle }}$ is the sole unknown, and can be varied to fit the experimental data using a least squares method. 


\subsubsection{TRPS Calibration Charge Methods (Methods II-IV)}

The $\zeta$-potential measurement methods described here seek to find a middle ground between mapping out an entire blockade (as in Section 4.1.2) and over-simplifying the analysis down to one duration measurement (as in references $[118,119])$. To do this the blockade is split into fractions so that a multi-point analysis can be used. For each blockade analysed, the method uses current values $I_{n}$ where $n=0.9,0.8,0.7,0.6,0.5,0.45,0.4$, $0.35,0.3,0.25,0.2,0.15,0.1,0.05$ corresponding to $90 \%, 80 \%, 70 \%$.. etc. of the blockade magnitude (for example $I_{0.9}=0.9 \times I_{1.0}$ ). In some measurements fewer values of $n$ were used (Section 4.2.1). It is assumed that the maximum blockade magnitude $\left(\Delta I_{\max }\right.$ or $\left.I_{1.0}\right)$ corresponds to the particle being in the pore entrance.

$T_{n}$ is then time between $\Delta I=I_{1.0}\left(T_{1.0}\right.$, given the value of $\left.0 \mathrm{~ms}\right)$ and $\Delta I=I_{n}$. The average particle velocity at $n\left(v_{n}\right)$ is proportional to $1 / T_{n}$. It is assumed that at each value of $n$, any particle has moved the same distance through the sensing zone as any other particle (regardless of size or charge) and therefore values of $T_{n}$ and $v_{n}$ can be directly compared between particles (Figure 4.4). For $\zeta$-potential measurements this compari- 


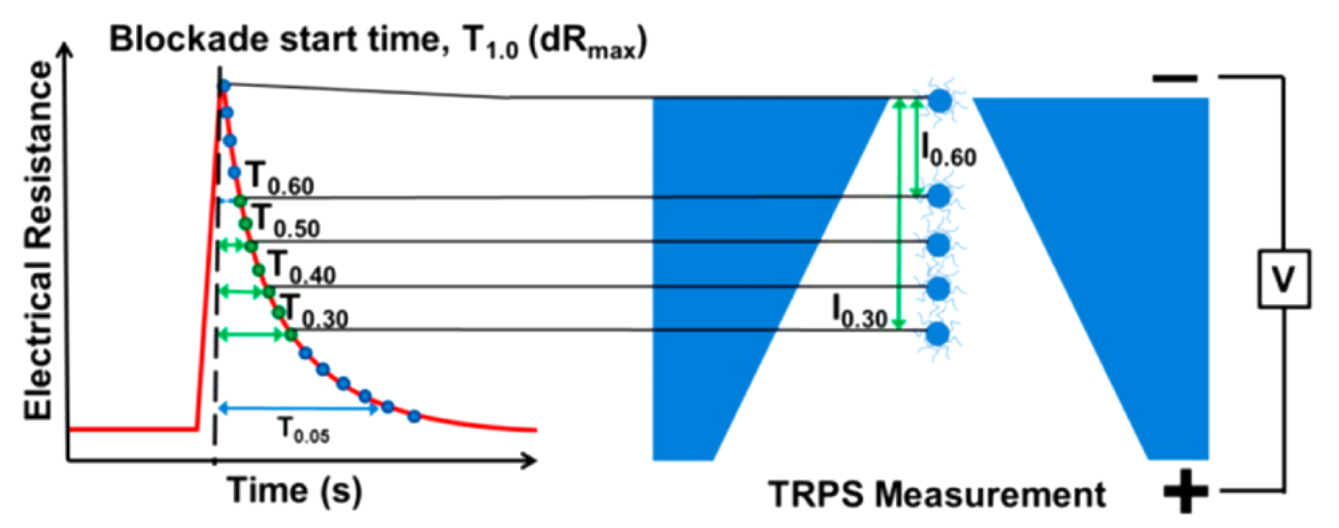

Figure 4.4: Schematic of the multi-point charge measurement model. Shows the relationship between $T_{n}$ and $I_{n}$ and particle position in the pore. Adapted from [220].

son will be between a calibration particle of known $\zeta$-potential $\left(\zeta_{\mathrm{Cal}}\right)$ and a sample particle of unknown $\zeta$-potential $\left(\zeta_{\text {Sample }}\right)$ in order to determine the latter.

Electrokinetic velocities $\left(v_{n}^{e l}\right)$ are related to particle $\zeta$-potential by Equation 4.9 assuming a linear relationship between $\zeta$-potentials as given in the Smoluchowski approximation:

$$
\frac{v_{n \text { Sample }}^{e l}}{v_{n_{\text {Cal }}}^{e l}}=\frac{\zeta_{n_{n e t} \text { Sample }}}{\zeta_{n_{n e t C a l}}}
$$

The net $\zeta$-potentials $\left(\zeta_{n e t}\right)$ for the sample and calibration particles are the difference between the respective $\zeta$-potentials and the $\zeta_{\text {pore }}$ : 


$$
\zeta_{\text {particle }}=\zeta_{\text {net }}+\zeta_{\text {pore }}
$$

$\zeta_{\text {pore }}$ can either be estimated based on literature or directly measured using streaming potential measurements (Chapter 5). $\zeta_{C a l}$ also needs to be measured independently.

As outlined in Chapter 2 the relevant particle transport mechanisms in TRPS are electrophoresis, electroosmosis and pressure-driven flow. The total particle velocity is the sum of the electrokinetic and pressure-driven flow velocities:

$$
v_{n}^{t o t}=v_{n}^{e l}+v_{n}^{p d f}
$$

where $v_{n}^{\text {tot }}$ and $v_{n}^{\text {pdf }}$ are the total and pressure-driven flow velocities respectively at each $n$. The electrokinetic velocity is a result of both electrophoretic and electroosmotic velocities. To find $\zeta_{\text {particle }}$, the electrophoretic velocity is measured, as this is determined by the charge on the particle. To do this the pressure-driven flow component needs to be characterised. This can be done by either calculating $v_{n}^{p d f}$ and subtracting it from the to- 
tal velocity (Equation 4.11), or by physically removing pressure from the system (Equation 4.11 where $v_{n}^{p d f}=0$ ). Once the pressure component has been removed the electrokinetic velocity of a particle can be compared to that of a calibration particle to obtain $\zeta_{\text {net Sample }}$ using Equation 4.9. Then the sample $\zeta$-potential can be calculated by subtracting $\zeta_{\text {pore }}$ (Equation 4.10):

$$
\zeta_{n \text { Sample }}=\frac{v_{n \text { Sample }}^{e l}}{v_{n_{\text {Cal }}}^{e l}} \times\left(\zeta_{\text {Cal }}-\zeta_{\text {pore }}\right)+\zeta_{\text {pore }}
$$

Three methods were used to remove the pressure-driven flow component to obtain the electrokinetic velocity: voltage sweep, pressure sweep and single pressure approaches.

\section{Voltage Sweep Method (Method II)}

In the voltage sweep method the pore is calibrated by measuring the dependence of $1 / T_{n}$ on voltage using standard carboxylated polystyrene particles with a known average $\zeta$-potential. This dependence is assumed to be linear, in accordance with the Nernst-Planck equation. Measurement of calibration particles is made at a minimum of three voltages $\left(V_{1}, V_{2}\right.$, and 
$V_{3}$ where $\left.V_{1}<V_{2}<V_{3}\right) .1 / T_{n}$ is averaged for each $n$ at each voltage. $v_{n}^{p d f}$ is determined from the $y$-intercept of the linear fits to the $1 / T_{n}$ vs. voltage data (Figure 4.5). $v_{n}^{p d f}$ is then subtracted from the total calibration particle velocity at $V_{3}, v_{n C a l}^{\text {tot }}$, to give $v_{n_{C a l}}^{e l}$ (Equation 4.11). Once $v_{n_{C a l}}^{e l}$ data are obtained the pore has been calibrated.

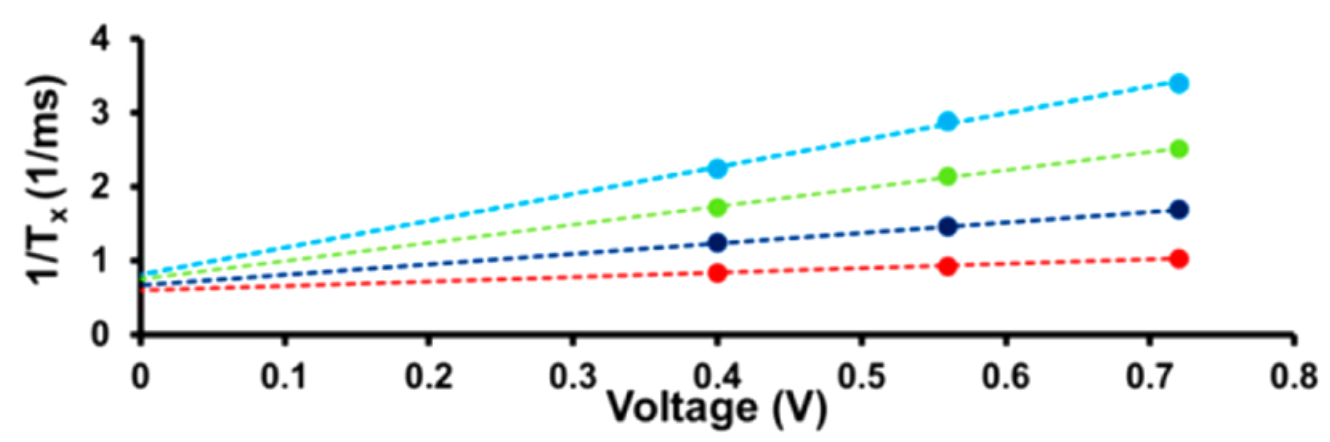

Figure 4.5: Schematic calibration plot of $1 / T_{n}$ against voltage. Different lines represent different $n$-values. Adapted from [220].

Measurement of the sample particles is taken at $V_{3}$ and $v_{n}^{\text {tot }}{ }_{\text {Sample }}$ is obtained. The pressure-driven flow component is assumed the same for calibration and sample measurements. Therefore, $v_{n}^{p d f}$ is subtracted from $v_{n}^{\text {tot }}{ }_{\text {Sample, }}$ again using Equation 4.11 , to give $v_{n \text { Sample }}^{\text {el }}$. Equation 4.12 can then be used to calculate $\zeta_{n \text { Sample }}$.

This method produces particle-by-particle data. The end result is a $\zeta$ potential value at each $n$ for each sample particle. The $\zeta$-potential is then averaged over a range of the $n$ values to give one $\zeta$-potential value per 
particle and can also be averaged over all of the particles measured to give an average $\zeta$-potential for the sample.

In iterations of this method $v^{e l}$ may be averaged over the voltages at which it was measured in order to give $v^{e l} / V$. This could be used to calibrate any sample measurement taken at any voltage, again using the Nernst-Plank equation. If pressure or vacuum is applied in the measurement $v^{p d f}$ may similarly be averaged over the pressures at which it was measured giving $v^{p d f} / P$ in a combination of Method II and Method III (described below). Again, this could be used in conjunction with any sample measurement taken at any pressure, as particle velocity is proportional to the applied pressure.

\section{Pressure Sweep Method (Method III)}

In the pressure sweep method the pore is calibrated by measuring the assumed linear dependence of $1 / T_{n}$ on pressure using standard carboxlyated polystyrene particles with a known average $\zeta$-potential. Measurement of calibration particles is made at a minimum of three pressures $\left(P_{1}, P_{2}\right.$ and $P_{3}$ where $\left.P_{1}<P_{2}<P_{3}\right) .1 / T_{n}$ is averaged for $n$ at each pressure. The 
electrokinetic component of the particle velocity at each $n\left(v_{n C a l}^{e l}\right)$ is determined from the $y$-intercept of the linear fits to the $1 / T_{n}$ vs. pressure data (Figure 4.6. Once $v_{n C a l}^{e l}$ is obtained the pore has been calibrated.

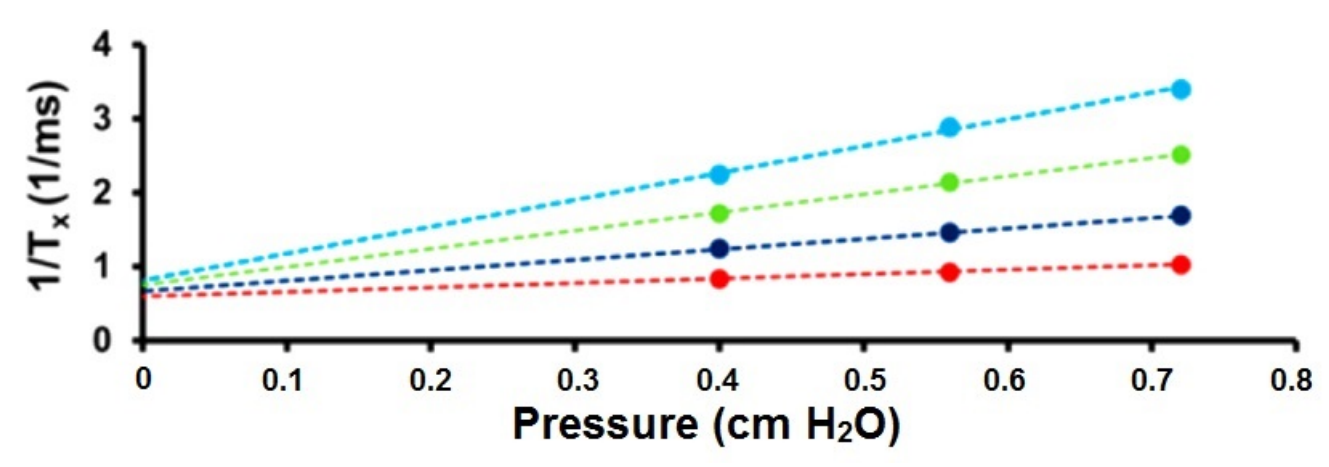

Figure 4.6: Schematic calibration plot of $1 / T_{n}$ against pressure. Different lines represent different $n$-values. Adapted from [220].

Measurements of the sample particles are then also made at a minimum of three pressures and the electrokinetic component of sample particle velocity at each $n\left(v_{n \text { Sample }}^{e l}\right)$ is also determined from the y-intercepts of the linear fits. At this stage the pressure-driven flow component has been removed from both the sample and calibration velocities. $\zeta_{n \text { Sample }}$ can then be calculated using Equation 4.12 .

This is an ensemble method. Because the average particle velocity must be taken at each pressure for both the sample and calibration the calibration particle velocities cannot be compared with individual sample block- 
ades.

\section{Single Pressure Method (Method IV)}

In the single pressure method the pore is calibrated by measuring $v_{n C a l}^{e l}$ at 0 net pressure i.e. directly finding the y-intercept in Figure 4.6. This is done by taking a single measurement at $-0.5 \mathrm{~cm} \mathrm{H}_{2} \mathrm{O}$ of applied pressure, which opposes the inherent pressure head. $v_{n}^{e l}$ is averaged over all particles measured in the calibration measurement to give $v_{n C a l}^{e l}$. Once this is obtained the pore is calibrated. Measurement of the sample particles is also made at 0 net pressure giving $v_{n \text { Sample }}^{e l}$ The pressure-driven flow component has been physically removed from both the sample and calibration velocities. Equation 4.12 can then be used to calculate $\zeta_{n \text { Sample }}$.

This method produces particle-by-particle data. The end result is a $\zeta$ potential value at each $n$ for each sample particle. The $\zeta$-potential is then averaged over a range of $n$ values to give one $\zeta$-potential value per particle and can also be averaged over all particles measured to give an average $\zeta$ potential for the sample. Example raw data for this Method is presented in Appendix C. 


\section{Summary of Methods}

Method I is based on a different model from Methods II-IV. It examines the entire blockade and requires pore geometry measurements. It requires calibration measurements, at different stretches, of a particle sample of known $\zeta_{\text {particle }}$. Once the pore is calibrated measurements of $\zeta_{\text {particle }}$ can be taken at any stretch, voltage or pressure.

Methods II-IV are based on the same multi-point model and the assumption that $I_{n}$ occurs at the same position regardless of particle properties or trajectory.

Method II requires calibration measurements at 3 voltages. Method III requires calibration measurements at 3 pressures. Method IV requires one calibration measurement at $P=-0.5 \mathrm{~cm} \mathrm{H}_{2} \mathrm{O}$. Methods II and IV produce particle-by-particle data. In contrast, Method III averages the electrokinetic velocity over all particles for both the calibration and sample particles and is therefore an ensemble technique.

One disadvantage of Method IV is that if sample particles are close in charge to the pore (i.e. $\zeta_{\text {net }} \sim 0$ ) then they may not be measurable at -0.5 $\mathrm{cm} \mathrm{H}_{2} \mathrm{O}$ and another method may have to be used. 


\begin{tabular}{|c|c|c|c|}
\hline $\begin{array}{l}\text { Method } \\
\text { I }\end{array}$ & $\begin{array}{l}\text { Systematic error } \\
\text { Assumptions: }\end{array}$ & Random error systematically applied & Random error \\
\hline & $\begin{array}{l}\text { - Uses Equation } 2.3 \text { assuming a conical pore of slowly } \\
\text { varying width } \\
\text { - } \Delta R_{\max } \text { occurs at } a, b \text { can be calculated from } \zeta_{C a l}\end{array}$ & $\begin{array}{l}\text { - } \zeta_{\text {particle and }} \zeta_{\text {pore }} \text { inputs measured using tech- } \\
\text { niques separate to TRPS } \\
\text { - } l \text { and its relationship with } X \text { is the same for all pores } \\
\text { - Geometric characterization for } a \text { and } b \text { across } X\end{array}$ & $\begin{array}{l}\text { - Particle-by-particle variation } \\
\text { - Error fitting model to data }\end{array}$ \\
\hline II & $\begin{array}{l}\text { Assumptions: } \\
\text { - Uses Equation } 2.3 \text { assuming a conical pore of slowly } \\
\text { varying width } \\
\text { - } I_{n} \text { occurs at the same position regardless of particle } \\
\text { properties or trajectory } \\
\text { - } v_{n} \propto V \text { right up to } V=0 \\
\text { - } v_{n}^{\text {pdf }} \text { applies to all particles }\end{array}$ & $\begin{array}{l}\text { - Uncertainty in finding the intercept }\left(v_{n}^{p d f}\right) \\
\text { - Uncertainty in averaging to get } v_{n}^{e l} \text { at each } n \\
\text { - } \zeta_{\text {particle }} \text { and } \zeta_{\text {pore }} \text { inputs measured using tech- } \\
\text { niques separate to TRPS }\end{array}$ & $\begin{array}{l}\text { - Uncertainty in each measurement } v_{n \text { Sample }}^{e l} \text { (can } \\
\text { be particle-by-particle) }\end{array}$ \\
\hline III & $\begin{array}{l}\text { Assumptions: } \\
\text { - Uses Equation } 2.3 \text { assuming a conical pore of slowly } \\
\text { varying width } \\
\text { - } I_{n} \text { occurs at the same position regardless of particle } \\
\text { properties or trajectory } \\
\text { - } v_{n} \propto P \text { right up to } P=0\end{array}$ & $\begin{array}{l}\text { - Uncertainty in finding the intercept for the calibra- } \\
\text { tion particles }\left(v_{C a l}^{e l}\right) \\
\text { - } \zeta_{\text {particle }} \text { and } \zeta_{\text {pore }} \text { inputs measured using tech- } \\
\text { niques separate to TRPS }\end{array}$ & $\begin{array}{l}\text { - Uncertainty in finding the intercept for each sample } \\
\left(v_{\text {Sample }}^{n}\right)\end{array}$ \\
\hline IV & $\begin{array}{l}\text { Assumptions: } \\
\text { - Uses Equation } 2.3 \text { assuming a conical pore of slowly } \\
\text { varying width } \\
\text { - } I_{n} \text { occurs at the same position regardless of particle } \\
\text { properties or trajectory }\end{array}$ & $\begin{array}{l}\text { - Uncertainty in applying "zero net pressure" } \\
\text { - Uncertainty in } v_{n C a l}^{e l} \\
\text { - } \zeta_{\text {particle and } \zeta_{\text {pore }} \text { inputs measured using tech- }} \\
\text { niques separate to TRPS }\end{array}$ & $\begin{array}{l}\text { - Uncertainty in each measurement } v_{n S a m p l e}^{e l} \text { (can } \\
\text { be particle-by-particle) }\end{array}$ \\
\hline
\end{tabular}

Table 4.1: Summary of uncertainities that apply to different measurement methods 


\subsubsection{Measurement Uncertainties}

As part of this investigation of $\zeta$-potential measurement using TRPS, it is important to understand experimental uncertainties so that the accuracy and precision of measurements can be ascertained. All experimental uncertainty is due to either random errors or systematic errors. Random errors may occur due to the measuring instruments (here the qNano, VPM, or Zetasizer) or in the environmental conditions (e.g. temperature). Random errors can be reliably estimated using repeat measurements and combined mathematically if there are multiple independent sources of random error. There are 2 types of random errors that have been identified in the measurement methods: random errors that apply systematically to many measurements and random errors that apply to individual measurements.

Systematic errors are caused by inaccuracies associated with the instrument itself, or else the models and experimental design. Systematic errors, unlike random errors, cannot be estimated by repeat measurements. An important systematic uncertainty in the described measurement methods comes from assumptions made in using Equation 2.3 to describe particle transport. Applying Equation 2.3 to TRPS assumes that dielectrophoresis 
and diffusion are negligible due to the low applied voltages and the applied pressure magnitudes. The gradient of the pore wall is assumed to be shallow enough that $\mathbf{E}$ and $\mathbf{Q}$ can take the same form as for an ideal cylinder (i.e. no component perpendicular to the pore axis). The pore is also assumed to be a circular cone geometry. Calculations take place in the thin EDL regime, under the Smoluchowski approximation in which particle size and pore diameter are much greater than the Debye length. As with most $\zeta$-potential measurement techniques, it is the particle mobility that is measured and this must be converted to a $\zeta$-potential using the Smoluchowski approximation (Equation 4.5).

The multi-point $\zeta$-potential measurement methods (Methods II-IV) also rely on assumptions and therefore introduce systematic errors. For example, it is assumed that $I_{n}$ occurs at the same position regardless of particle size, charge, type, or trajectory.

Identified sources of random and systematic uncertainty for each measurement method are collated in Table 4.1. Examples of random error analysis can be found in Tables 4.2-4.3, 4.5, and 5.1. Ultimately, the uncertainty of interest is that of the absolute $\zeta$-potential measurements such as with before and after on-bead experiments where the difference between par- 
ticles is being examined. However, more robust science can be done to understand the information that can be obtained from a $\zeta$-potential value.

\subsection{Results and Discussion}

\subsubsection{Comparing Method I and Method II}
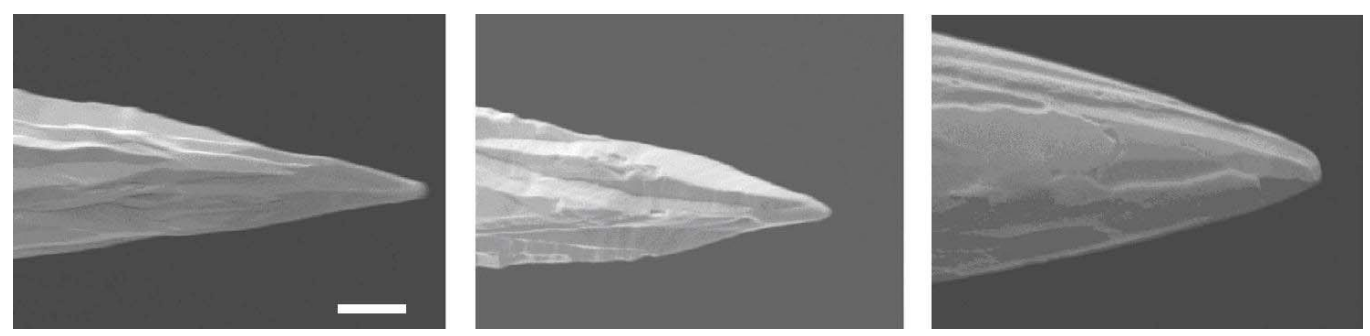

Figure 4.7: SEM of tungsten needles used to make pores. Left to right, needles A, B and C. The scale bar $(1 \mu \mathrm{m})$ applies to all images.

In this Section, Method I, which fits data to the entire resistive pulse trace, is compared to Method II, which examines discrete points along the resistive pulse. The effects of voltage and specimen fabrication on $\zeta$ potential measurements are explored using both methods.

Figure 4.8 plots particle $\zeta$-potential measurements as a function of applied potential, with data obtained from experiments using two particle sets (B and E, Table 3.3) suspended in SEB and five pore specimens (Ta- 
ble 3.2, pores (i)-(v)) which were fabricated using three different needles (Figure 4.7). Pores (i) and (ii) were fabricated using needle A, pore (iii) was fabricated using needle B and pores (iv) and (v) were fabricated using needle C.

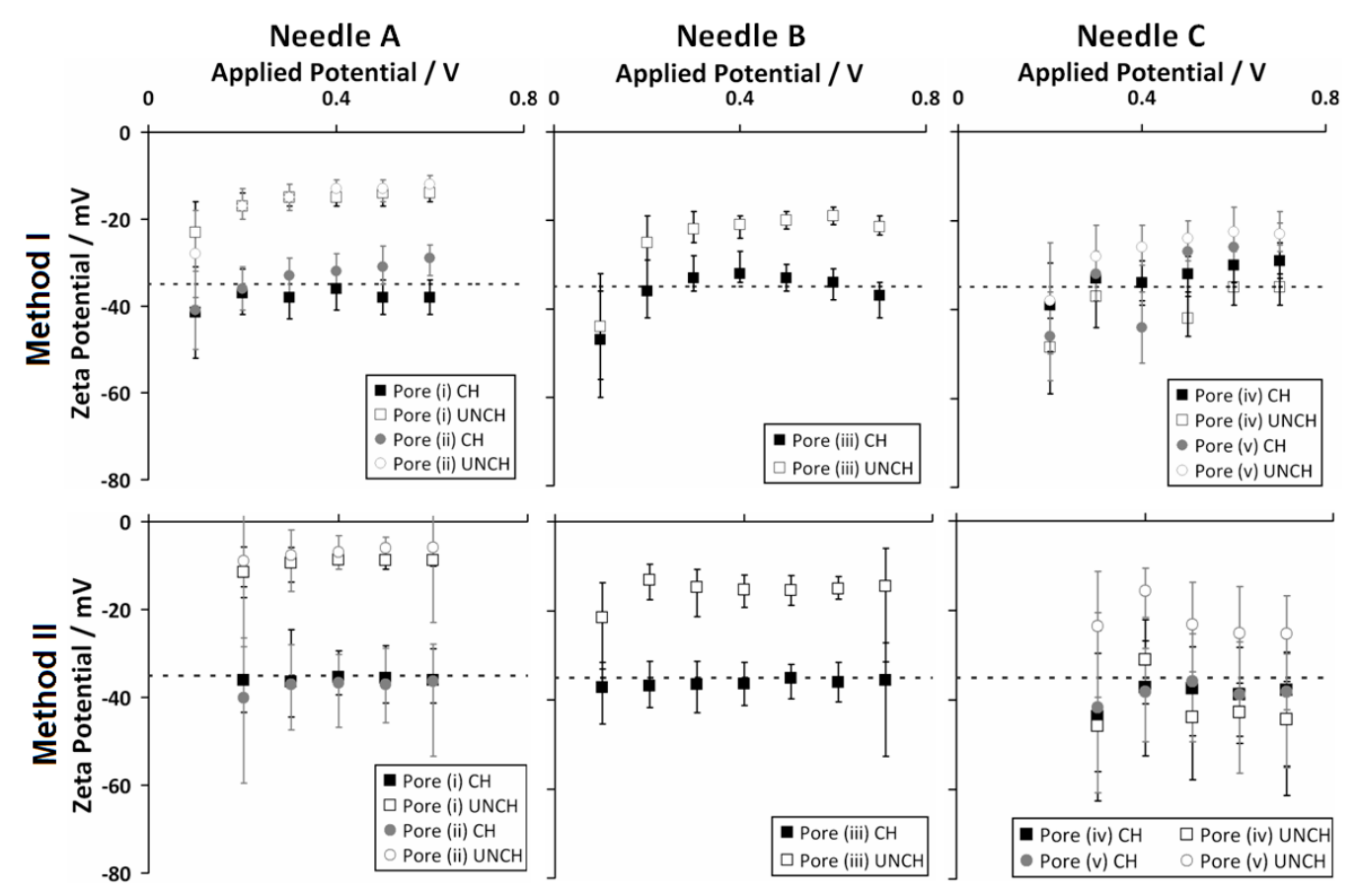

Figure 4.8: $\zeta$-potential values for charged (" $\mathrm{CH}^{\prime}$, set B) and uncharged ("UNCH", set E) particle sets, derived using two analysis methods (Section 4.1.3), plotted as a function of potential applied across the membrane. The five pores used (Table 3.3 pores (i)-(v)) were fabricated by three different needles (Figure 4.7). Dashed horizontal lines indicate the calibration value for charged particles at $0.6 \mathrm{~V}$ applied potential $(-35 \mathrm{mV})$. Error bars indicate the upper and lower quartile values for each particle population.

The charged particle set was used for calibration of both methods, with an assigned $\zeta$-potential of $-35 \mathrm{mV}$ (as specified by the supplier). Calibra- 
tions were carried out at $0.6 \mathrm{~V}$ applied potential for Method I and 0.2-0.6 V for Method II. Because the charged particle sets were used for calibration, it is expected that the $\zeta$-potential values for these particles should be -35 $\mathrm{mV}$, indicated by the horizontal dashed lines in Figure 4.8 .

When using both methods, charged and uncharged particles could be distinguished using pores (i) and (ii), manufactured using Needle A, and pore (iii), manufactured using Needle B. However, measurements from pores (iv) and (v), manufactured using Needle C, revealed little difference in $\zeta$-potential between particle sets within their interquartile ranges.

Overall, the two analysis methods produce similar results, but it appears that Method II produces a larger spread of data. For example, the $\zeta$ potential on the uncharged particle set appears higher (more positive) for all pores when using Method II. For pore (v), the spread of data is greater using Method II than Method I. It is proposed that this difference is due to the sampling of discrete points along the resistive pulse in Method II which gives better resolution of the differences in durations, as opposed to fitting to the entire pulse in Method I where these differences in durations could be blurred. 
A key reason for variability in the results obtained using different pores is geometric variation from specimen to specimen, intentional or otherwise. The pore geometry is dependent on the characteristics of the tungsten needle, puncture control and the material properties [52]. Pores manufactured using the same needle, similar penetration settings and the same membrane material are therefore expected to exhibit similar properties, as observed in Figure 4.8. Needles A and B are the sharpest of the 3 needles whereas Needle $C$ can be seen to be much broader (Figure 4.7). It is therefore proposed that the geometry of pores manufactured using sharper needles is closer to the model used for calculations, being perhaps more regularly shaped and closer to a truncated linear cone, leading to increased precision and accuracy in the results.

Results are less consistent at applied potentials below $0.3 \mathrm{~V}$ and above $0.6 \mathrm{~V}$, defining a working range for apparatus, pores, applied stretches and particles similar to those used. At low potentials, the electrokinetic component of particle motion becomes dominated by pressure-driven flow, so that the electrophoretic mobility of each particle is difficult to discern. At high potentials, electronic noise increases.

As an overall perspective on the two analysis methods, the major chal- 
lenge for Method I is definition of the pore geometry, and the change in geometry as the pore is stretched or relaxed. As defined above, Method I differs slightly from the original methodology proposed by Kozak et al. $[46,82]$ because a calibration particle set of known $\zeta$-potential is used to define $b$, rather than using the baseline current. This change reduces the calculation's dependence on specific pore geometry, but adds the requirement for calibration particles. Method II, which also requires calibration particles, removes the need for well-defined pore geometry, instead making an assumption regarding the similarity of resistive pulse shapes for similar experimental conditions. Although the accuracy of this assumption can be explored further, our data for pore (v) suggest that Method II is superior for distinguishing particles of different zeta potential when the geometry of the pore is more difficult to define.

\section{Uncertainties}

Uncertainty calculations were conducted for Method II to provide an example of the magnitude of uncertainties in absolute values of $\zeta_{\text {particle }}$ and where they are introduced. The random error in $\zeta_{\text {Sample }}$ measurements using Method II for charged particles on pore (iii) was calculated. These 
Table 4.2: Random error in $\zeta_{n S a m p l e}$ for measurements of charged particles on pore (iii) using Method II

\begin{tabular}{|c|c|c|c|c|c|c|c|}
\hline$n$ & $0.1 \mathrm{~V}$ & $0.2 \mathrm{~V}$ & $0.3 \mathrm{~V}$ & $0.4 \mathrm{~V}$ & $0.5 \mathrm{~V}$ & $0.6 \mathrm{~V}$ & $0.7 \mathrm{~V}$ \\
\hline 0.3 & $104.4 \%$ & $109.9 \%$ & $58.9 \%$ & $49.6 \%$ & $40.9 \%$ & $39.2 \%$ & $41.5 \%$ \\
\hline 0.4 & $121.4 \%$ & $96.6 \%$ & $59.1 \%$ & $53.8 \%$ & $46.2 \%$ & $44.0 \%$ & $44.7 \%$ \\
\hline 0.5 & $159.8 \%$ & $89.3 \%$ & $59.7 \%$ & $54.7 \%$ & $46.3 \%$ & $43.3 \%$ & $43.1 \%$ \\
\hline 0.6 & $241.6 \%$ & $91.9 \%$ & $69.3 \%$ & $55.0 \%$ & $46.7 \%$ & $44.9 \%$ & $44.4 \%$ \\
\hline 0.7 & $514.1 \%$ & $107.2 \%$ & $76.8 \%$ & $58.1 \%$ & $50.1 \%$ & $47.1 \%$ & $48.2 \%$ \\
\hline 0.8 & $1796.5 \%$ & $116.9 \%$ & $53.0 \%$ & $66.1 \%$ & $57.2 \%$ & $54.7 \%$ & $57.5 \%$ \\
\hline 0.9 & $485.4 \%$ & $136.1 \%$ & $103.6 \%$ & $85.2 \%$ & $78.4 \%$ & $78.6 \%$ & $83.7 \%$ \\
\hline
\end{tabular}

Table 4.3: Random error in $v_{n}^{p d f}$ for $\zeta$-potential measurements of charged particles on pore (iii) using Method II

\begin{tabular}{|c|c|}
\hline$n$ & $\sigma_{n}^{p d f}$ \\
\hline 0.3 & $4.6 \%$ \\
\hline 0.4 & $6.3 \%$ \\
\hline 0.5 & $2.3 \%$ \\
\hline 0.6 & $3.4 \%$ \\
\hline 0.7 & $5.4 \%$ \\
\hline 0.8 & $5.4 \%$ \\
\hline 0.9 & $5.3 \%$ \\
\hline
\end{tabular}

errors were calculated without including errors from the calibration $\zeta$ potential and $\zeta_{\text {pore }}$ because these values were estimated rather than measured. Combining the uncertainty in $v_{n S a m p l e}^{e l}$ and $v_{n C a l}^{e l}$ using Equation 4.12 gives the percentage uncertainty in $\zeta_{n \text { Sample. }}$ The error in these velocities can be large as they come from Equation 4.11 and therefore the error propagation rule for sums is used. These uncertainties at each $n$ and $V$ can be found in Table 4.2. It can be seen that the error in $\zeta_{n S a m p l e}$ is lowest at 
high voltages and values of $n$ close to 0.3 . The uncertainties are high at low voltages because the blockade size gets close to the size of the noise which introduces error into $I_{n}$ especially if $n$ is large as $I_{n}$ is then closer to the baseline. These uncertainty calculations could be extended with the addition of measured $\zeta_{C a l}$ and $\zeta_{\text {pore }}$ values and their associated errors.

The uncertainty in $v_{n}^{p d f}\left(\sigma_{n}^{p d f}\right)$ was also calculated. $\sigma_{n}^{p d f}$ comes from the uncertainty in finding the $\mathrm{y}$-intercept of the voltage vs. $v_{n}$ plots. The percentage uncertainties in $v_{n}^{p d f}$ at each $n$ are shown in Table 4.3. This is a systematically applied random error as it applies to the calibration measurements which are used to measure both charged and uncharged particles.

To calculate an average $\zeta$-potential value for the sample $\zeta_{\text {Sample }}$ must be averaged over $n$ at a chosen $V$. The values of $n$ which are being averaged over can be chosen based on the lowest observed uncertainties in previous calculations. For experiments in Section 4.2.1 blockades were measured at $n=0.3,0.4,0.5,0.6,0.7,0.8$, and 0.9 and averaged over all values of $n$. At $V=0.7 \mathrm{~V}$, when averaging over $n=0.3-0.9$ there is only a $0.8 \%$ standard error in $\zeta_{\text {Sample. }}$. 
To summarise, for absolute values of $\zeta_{\text {particle }}$ there can be large errors (Table 4.2), the magnitude of these errors is partially due calculations using Equation 4.11. These errors could potentially be larger if the uncertainty in $\zeta_{\text {pore }}$ and $\zeta_{\text {Cal }}$ was included. This is the main error as it is much larger than the uncertainty in finding $v_{n}^{p d f}$ (Table 4.3) or due to averaging across $n$.

The data points and error bars presented in Figure 4.8 are median values and interquartile ranges over minimum 500 particles with no other uncertainties included.

\subsubsection{DLS Measurements}

As with TRPS size and concentration measurements, ideally the system would be calibration-free. However, defining pore geometry is complicated and it is much more straightforward to work with calibration particles. For all charge measurements taken in this research calibration is required. Broader application of these methods would require a range of different sized calibration particles with defined charges, in a number of electrolytes. In this Section, calibration values were obtained using DLS. 


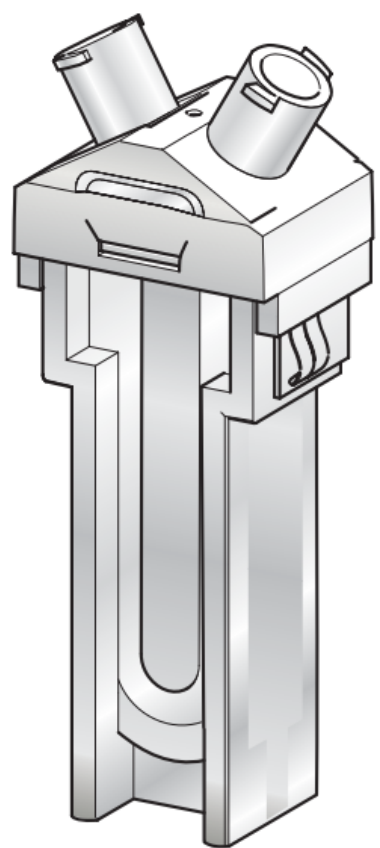

Disposible cell

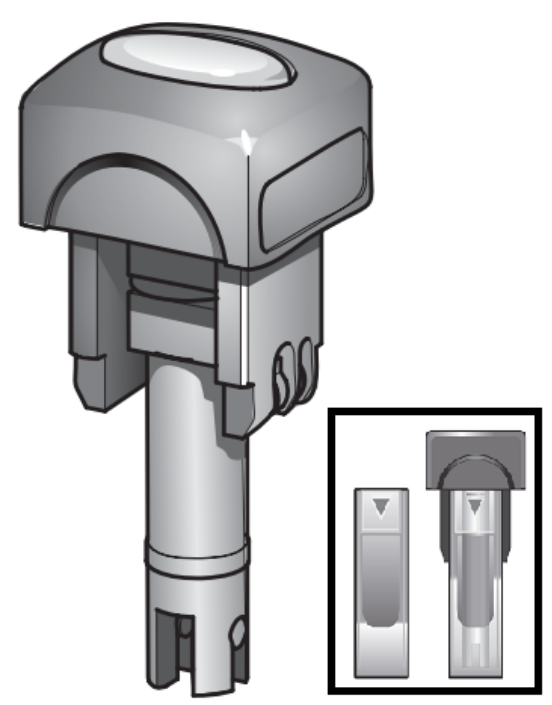

Dip cell

Figure 4.9: Schematics of a DFCC and a UDC with an inset showing how the UDC works with square based cells. Adapted from [224]. 
As outlined in Chapter 3 the instrument used for DLS measurements in this Thesis is the Malvern Zetasizer Nano ZS. The two main types of $\zeta$-potential measurement cells used in conjunction with the Zetasizer are DFCCs and UDCs (Figure 4.9). Initial experiments studied the difference between the two cell types.

DFCCs (Figure 4.8) consist of a polycarbonate body and gold plated beryllium/copper electrodes. They have been designed to be used for a single measurement or series of measurements, then discarded rather than cleaned. UDCs (Figure 4.8) are used with disposable polystyrene cells (for aqueous samples) or reusable glass square aperture cells (for non-aqueous samples). The electrodes are made of palladium, casings are made of natural polyetheretherketone (PEEK), and the upper contacts are phosphor bronze with nickel plating [224].

In these experiments a single DFCC was used for the 5 measurements for each electrolyte. Disposable polystyrene cells were used with the UDC. One disposable polystyrene cell was used per measurement. It was found that DFCCs gave a less negative $\zeta$-potential for all particle sets, in 3 different electrolytes (SEB, PBS and HEPES), apart from the Malvern Standard (MS) in SEB and PBS (Figure 4.10). This suggests that DFCCs are less sen- 

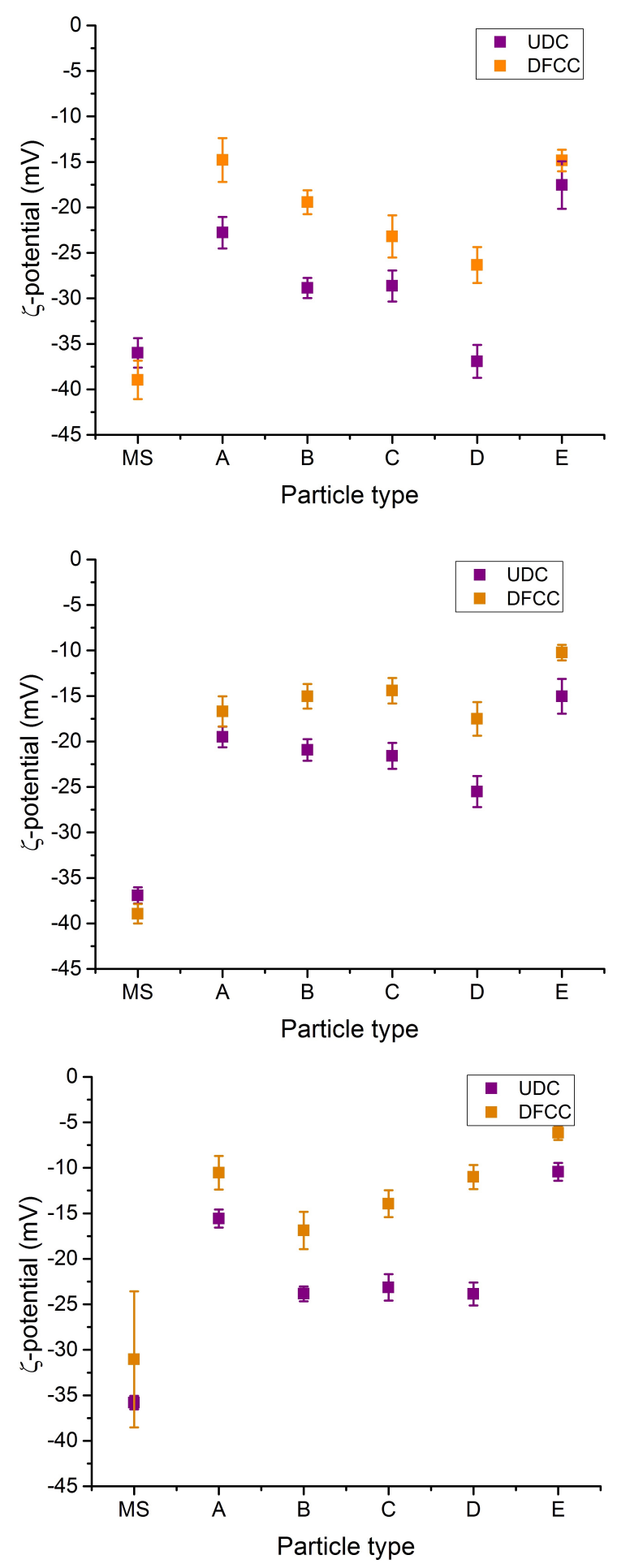

Figure 4.10: Plots showing particle charge measured using the UDC and DFCC in SEB (upper), PBS (middle) and HEPES (lower). Particle set details can be found in Table 3.3. 
sitive than the UDC. DFCC electrodes were observed to discolour after more than one use, suggesting that the electrodes were wearing out and this was affecting the $\zeta$-potential measurements. Due to these results it was decided that the UDC was the best option for measuring particle $\zeta$ potentials.

Table 4.4: Calibration particle $\zeta$-potentials measured using DLS

\begin{tabular}{|c|c|c|c|}
\hline Particle Type & Nominal size $(\mathrm{nm})$ & $\zeta$ in SEB $(\mathrm{mV})$ & $\zeta$ in PBS $(\mathrm{mV})$ \\
\hline \multicolumn{4}{|c|}{ CPN-particles } \\
\hline CPN100 & 100 & $-24.6 \pm 1.2$ & $-11.9 \pm 1.4$ \\
\hline CPN200 (E) & 200 & $-19.9 \pm 1.1$ & $-12.1 \pm 0.7$ \\
\hline CPN400 & 400 & $-19.1 \pm 0.9$ & $-15.4 \pm 0.9$ \\
\hline CPN800 & 800 & $-18.2 \pm 1.8$ & $-14.7 \pm 0.5$ \\
\hline CPN1000 & 1000 & $-17.9 \pm 1.5$ & $-15.0 \pm 0.6$ \\
\hline CPN2000 & 2000 & $-26.2 \pm 1.5$ & $-14.2 \pm 0.7$ \\
\hline \multicolumn{4}{|c|}{ CPC-particles } \\
\hline CPC100 & 100 & $-38.0 \pm 1.6$ & $-15.2 \pm 1.8$ \\
\hline CPC200 (B) & 200 & $-46.8 \pm 3.1$ & $-18.2 \pm 1.5$ \\
\hline CPC400 & 400 & $-56.2 \pm 2.4$ & $-45.4 \pm 1.2$ \\
\hline CPC800 & 800 & $-49.2 \pm 2.6$ & $-39.5 \pm 5.6$ \\
\hline CPC1000 & 1000 & $-65.0 \pm 1.5$ & $-50.3 \pm 2.7$ \\
\hline CPC2000 & 2000 & $-54.5 \pm 2.2$ & $-47.0 \pm 2.7$ \\
\hline
\end{tabular}

Calibration particles are used in TRPS size, concentration and charge experiments. They are usually CPC or CPN spheres. Results from DLS measurements of Izon Science calibration particles CPC100, 200, 400, 800, 1000 and 2000 as well as their CPN equivalents are shown in Table 4.4. Measurements were taken in the two most commonly used TRPS electrolytes, SEB and PBS. This table will be useful to researchers doing charge 
measurements using TRPS.

\subsubsection{Method III and IV with Varied Electrolytes}

$\zeta$-potential is affected by ionic strength $I S$ via the Debye length $\kappa^{-1}$. At lower ionic strengths the EDL is compressed resulting in movement of the shear plane closer to the surface and therefore a lower $\zeta$-potential (Equation 4.2 and Figure 4.1).

The $\zeta$-potential of a range of nominally $200 \mathrm{~nm}$ particles with different surface charges was investigated using DLS and TRPS. The $\zeta$-potential was first investigated in 3 different electrolytes using Method III and then in a single electrolyte at a range of dilutions using Method IV. The results of these measurements are shown in Figures 4.11 and 4.12.

The manufacturer's charge specifications for particle sets A-E can be found in Table 3.3. D is expected to be the most charged particle set, followed by C, B, A and then E. Especially for DLS it can be seen that the particles generally follow this order of charge, although sometimes particles are very close in charge and hard to distinguish between. The TRPS results are slightly less clear but still the general pattern of particle charge 


\section{DLS}

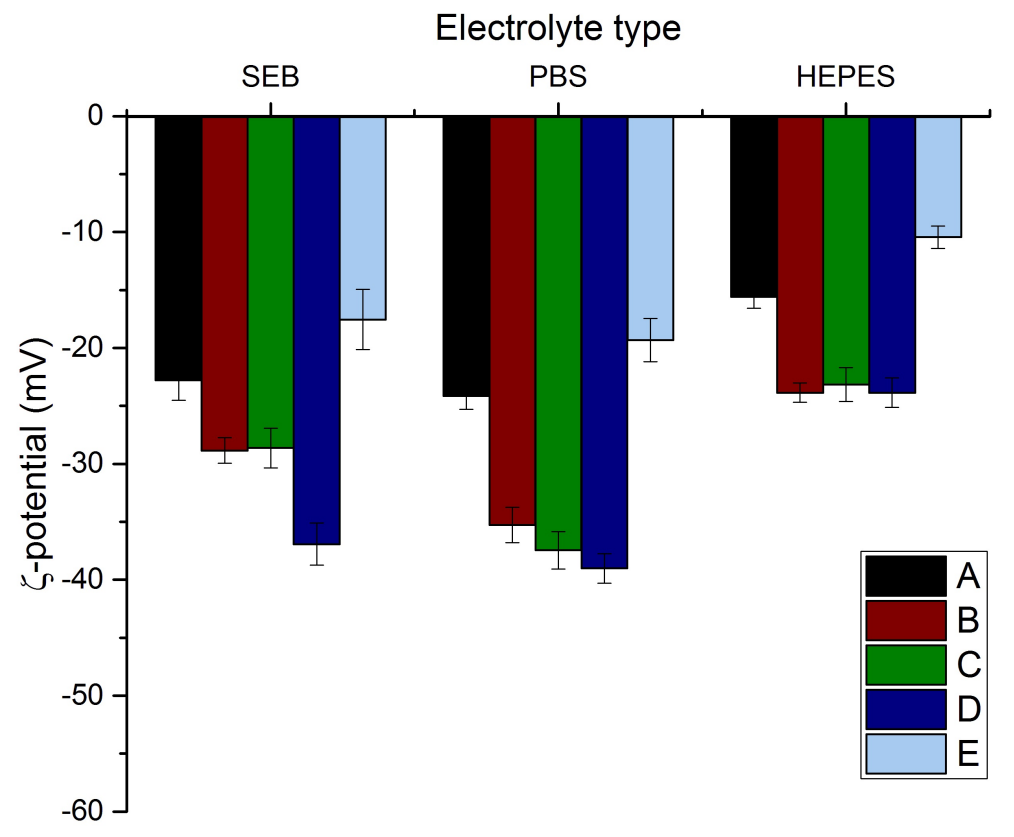

\section{TRPS}

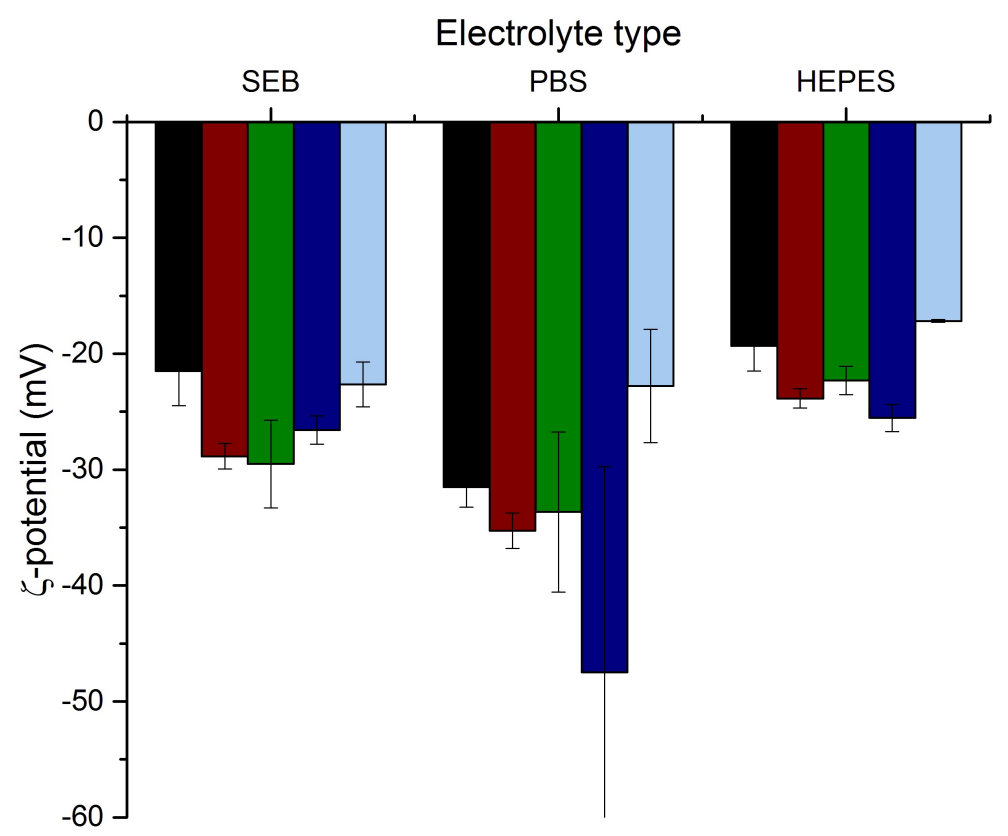

Figure 4.11: $\zeta$-potential of 5 particle sets measured in 3 different electrolytes by DLS (upper) and TRPS Method III (lower). Errors are the standard deviation over 10 and 3 measurements respectively. 

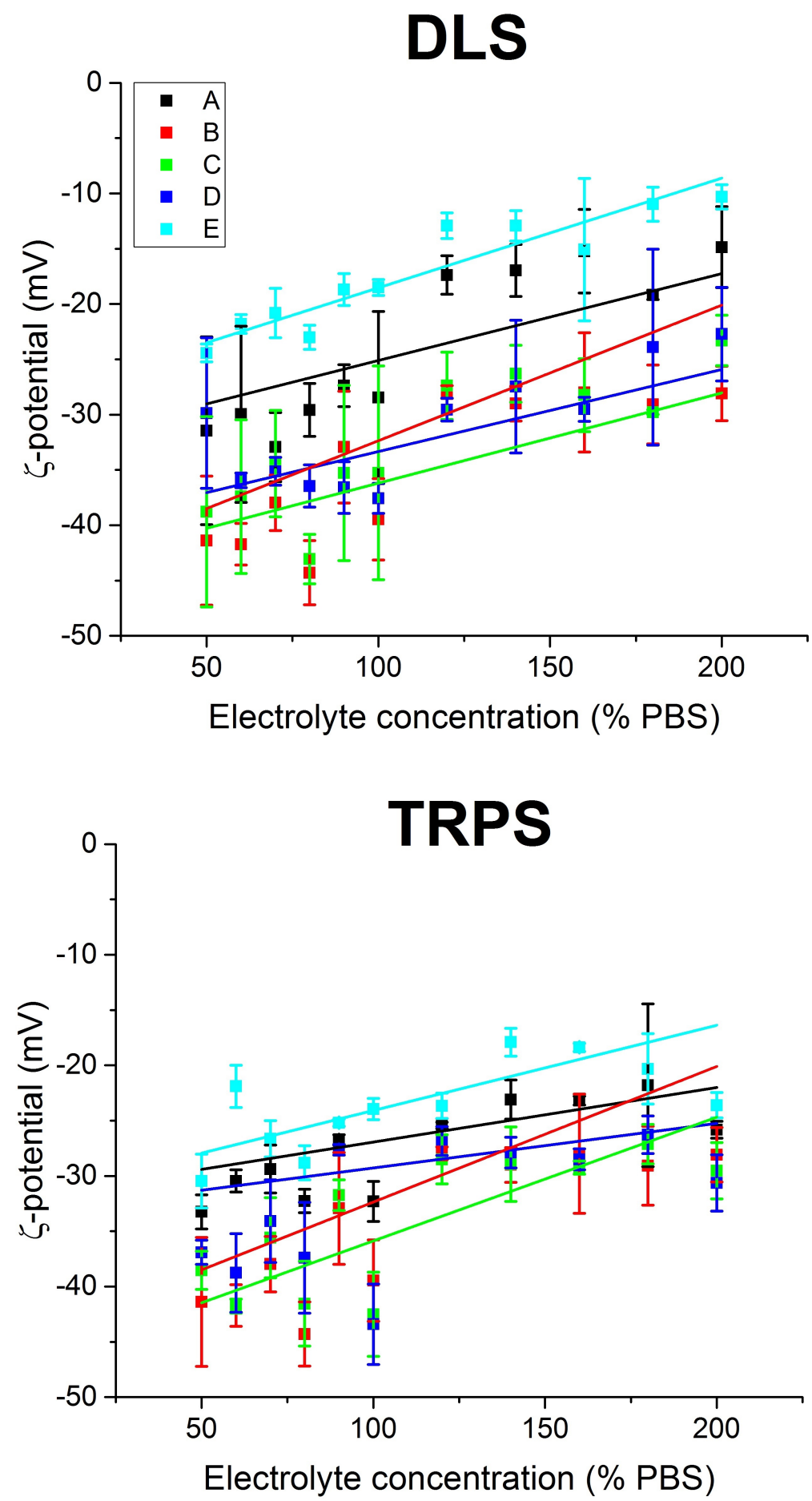

Figure 4.12: $\zeta$-potential of 5 particle sets measured in 11 different electrolyte concentrations by DLS (upper) and TRPS Method IV (lower). Errors are the standard deviation over 10 and 3 measurements respectively. Lines are linear fits to the data. 
is as expected. The large error bars seen for TRPS, here representing the standard deviation over 3 repeat measurements, show that it's easier for errors and anomalies to appear in TRPS results because there are more manually controlled inputs (e.g. stretch, voltage, exact pore used). There were less repeats for the TRPS measurements than for DLS which can also result in larger error bars. For both TRPS and DLS the system was completely re-set between measurements. For TRPS this involved disassembly and cleaning of the pore and fluid cell and introduction of a fresh sample, for DLS this involved cleaning of the UDC and a fresh sample in a new cell. The more complex re-setting of the TRPS system may have also contributed in the larger standard deviation between measurements.

There was no clear trend or difference observed between electrolytes (Figure 4.11). Therefore, this investigation turned to examining electrolyte concentration variation within the same electrolyte (PBS, Figure 4.12). An increase (becoming less negative) in $\zeta$-potential with increasing electrolyte concentration is seen for all particle sets, for both DLS and TRPS measurements (Figure 4.12). Again, the trend with nominal surface charge was clearer for DLS than for TRPS.

The $\zeta_{\text {pore }}$ value for measurements in Figure 4.11 was obtained using ex- 
ternal streaming potentiometer measurements [111]. $\zeta_{\text {pore }}$ values for measurements in Figure 4.12 were estimated based on manufacturer's suggestions. These measurements were improved upon in Chapter 5.

\section{Uncertainties}

Uncertainty calculations were conducted for Method IV to provide an example of the magnitude of uncertainties in absolute values of $\zeta_{\text {particle }}$ and where they are introduced. The random error in $\zeta_{\text {Sample }}$ measurements using Method IV for particle sets A, C, D, and E in $50 \%$ PBS was calculated. These errors were calculated without including errors from $\zeta_{\text {pore }}$ as this value was estimated rather than measured. Combining the uncertainties in $v_{n S a m p l e}^{e l}, v_{n C a l}^{e l}$ and $\zeta_{C a l}$ using Equation 4.12 gives the percentage uncertainty in $\zeta_{n \text { Sample. }}$. These uncertainties at each $n$ for the 4 particle sets can be found in Table 4.5. It can be seen that the error in $\zeta_{n \text { Sample }}$ is lowest at values of $n$ between $0.2-0.45$. The uncertainty is similar across particle types as all particles are similar in size and thus the blockade size does not approach the baseline. Again, when $n$ is larger $I_{n}$ is closer to the baseline current and therefore more error is introduced. 
It can be seen that compared to the errors presented in Method II (Tables 4.2-4.3) there is relatively less random error. Although systematically applied random error measurements are similar, particle-by-particle errors are lower using this method. It is important to remember that the error in finding $P=0$ has not been accounted for. The addition of $\zeta_{\text {pore }}$ measurements and uncertainties would further improve Method IV and the understanding of uncertainties (Chapter 5).

Table 4.5: Random errors in $\zeta_{n \text { Sample }}$ for particle sets A, C, D, and E in $50 \%$ PBS using Method IV.

\begin{tabular}{|c|c|c|c|c|}
\hline$n$ & $\mathrm{~A}$ & $\mathrm{C}$ & $\mathrm{D}$ & $\mathrm{E}$ \\
\hline 0.05 & $52.0 \%$ & $45.6 \%$ & $54.7 \%$ & $49.1 \%$ \\
\hline 0.1 & $51.7 \%$ & $42.8 \%$ & $49.7 \%$ & $46.0 \%$ \\
\hline 0.15 & $49.1 \%$ & $42.2 \%$ & $48.4 \%$ & $44.6 \%$ \\
\hline 0.2 & $47.6 \%$ & $40.4 \%$ & $48.2 \%$ & $39.9 \%$ \\
\hline 0.25 & $45.2 \%$ & $38.6 \%$ & $43.9 \%$ & $43.5 \%$ \\
\hline 0.3 & $44.1 \%$ & $37.7 \%$ & $41.6 \%$ & $40.9 \%$ \\
\hline 0.35 & $44.3 \%$ & $37.6 \%$ & $40.5 \%$ & $39.5 \%$ \\
\hline 0.4 & $44.0 \%$ & $38.6 \%$ & $40.2 \%$ & $39.1 \%$ \\
\hline 0.45 & $45.2 \%$ & $39.9 \%$ & $41.2 \%$ & $40.5 \%$ \\
\hline 0.5 & $47.7 \%$ & $41.3 \%$ & $41.9 \%$ & $41.9 \%$ \\
\hline 0.6 & $49.9 \%$ & $42.6 \%$ & $44.3 \%$ & $46.6 \%$ \\
\hline 0.7 & $51.7 \%$ & $44.1 \%$ & $46.8 \%$ & $49.8 \%$ \\
\hline 0.8 & $56.0 \%$ & $46.3 \%$ & $49.7 \%$ & $57.1 \%$ \\
\hline 0.9 & $112.8 \%$ & $55.8 \%$ & $110.0 \%$ & $63.8 \%$ \\
\hline
\end{tabular}

To calculate an average $\zeta$-potential value for the sample $\zeta_{\text {Sample }}$ must be averaged over $n$. The values of $n$ to be averaged over can be chosen based on the lowest observed uncertainties in previous calculations. For 
experiments in this Chapter using Method IV, blockades were measured at $n=0.05,0.1,0.15,0.2,0.25,0.3,0.35,0.4,0.45,0.5,0.6,0.7,0.8$, and 0.9 and almost always averaged over $n=0.2-0.45$. For particle sets $\mathrm{A}, \mathrm{C}, \mathrm{D}$ and $\mathrm{E}$ when averaging over $n=0.2-0.45$ there is only a $1.3,1.3,2.4$, and 1.3 $\%$ error respectively, in $\zeta_{\text {sample }}$.

To summarise, for absolute values of $\zeta_{\text {particle }}$ there can be large errors (Table 4.2), the magnitude of these errors is partially due calculations using Equation 4.12 which in this method includes the uncertainty due to $\zeta_{\text {Cal }}$. These errors could potentially be larger if the uncertainty in $\zeta_{\text {pore }}$ was included. This is the main error as it is much larger than the uncertainty due to averaging across $n$.

Data points and error bars in Figures 4.11 and 4.12 are the average and standard deviation of mean values over 3 measurement sets each containing minimum 500 particles. These error bars therefore represent the random error in $\zeta_{\text {sample }}$ without the inclusion of the other random errors outlined in Table 4.1. 


\subsubsection{Methods III and IV with Varied $\mathrm{pH}$}

$\mathrm{pH}$ is another important property of the electrolyte that influences the $\zeta$ potential of suspended particles. In this case, the surface charge density of the surface is altered by change in $\mathrm{pH}$. Particles in suspension, such as the carboxylated polystyrene spheres used here, develop their surface charge by charge transfer between the particle surface and the solution they are suspended in. Particle surface hydration and acid dissociation of the carboxyl group leads to a negative charge on the particle surface, and thus positive counter ions balancing this charge in the EDL. A more basic $\mathrm{pH}$ results in an increase in acid dissociation and a more negative surface charge. This in turn results in more cations moving into the shear plane to counterbalance this charge and a more negative $\zeta$-potential. A positive charge arises from the addition of a proton to the carboxyl group, which results in anions counter balancing the surface charge and a positive $\zeta$-potential. The equations provided below show that changes in the concentration of acid or base affect the surface charge and hence the $\zeta$ potential. PS is representative of the polystyrene particle.

$$
\mathrm{PS}-\mathrm{COOH}+\mathrm{H}_{2} \mathrm{O} \longrightarrow \mathrm{PS}-\mathrm{COO}^{-}+\mathrm{H}_{3} \mathrm{O}^{+}
$$


$\mathrm{PS}-\mathrm{COOH}+\mathrm{H}_{3} \mathrm{O}^{+} \longrightarrow \mathrm{PS}-\mathrm{CO}(\mathrm{OH})_{2}{ }^{+}+\mathrm{H}_{2} \mathrm{O}$

The isoelectric point, or point of zero charge, is the $\mathrm{pH}$ at which there is no acid dissociation and the carboxylated polystyrene spheres are completely neutral with no need for counter ions to balance the charge. The $\zeta$-potential is therefore 0 . For carboxylated polystyrene particles this has been found to be at a pH less than 2 [225]. For standard TRPS measurements, lack of surface charge is problematic as it increases aggregation and makes particles difficult to measure.

The final experiments in this Chapter involved varying the $\mathrm{pH}$. Unfortunately, it was discovered that TRPS only works well over a small range of pHs. The addition of surfactant improves TRPS measurements but shields some particle charge, altering observed trends so surfactant was not used for TRPS measurements in this Chapter.

Figure 4.13 shows $\zeta$-potential measurements for the various particle sets using DLS for $100 \mathrm{mM} \mathrm{KCl}$ with and without $0.03 \% \mathrm{v} / \mathrm{v}$ Triton-X. The expected pattern is that with decreasing $\mathrm{pH}$, particle $\zeta$-potential will become less negative. However, this pattern is disrupted by the introduction of surfactant. No TRPS charge measurements were taken in this section of 

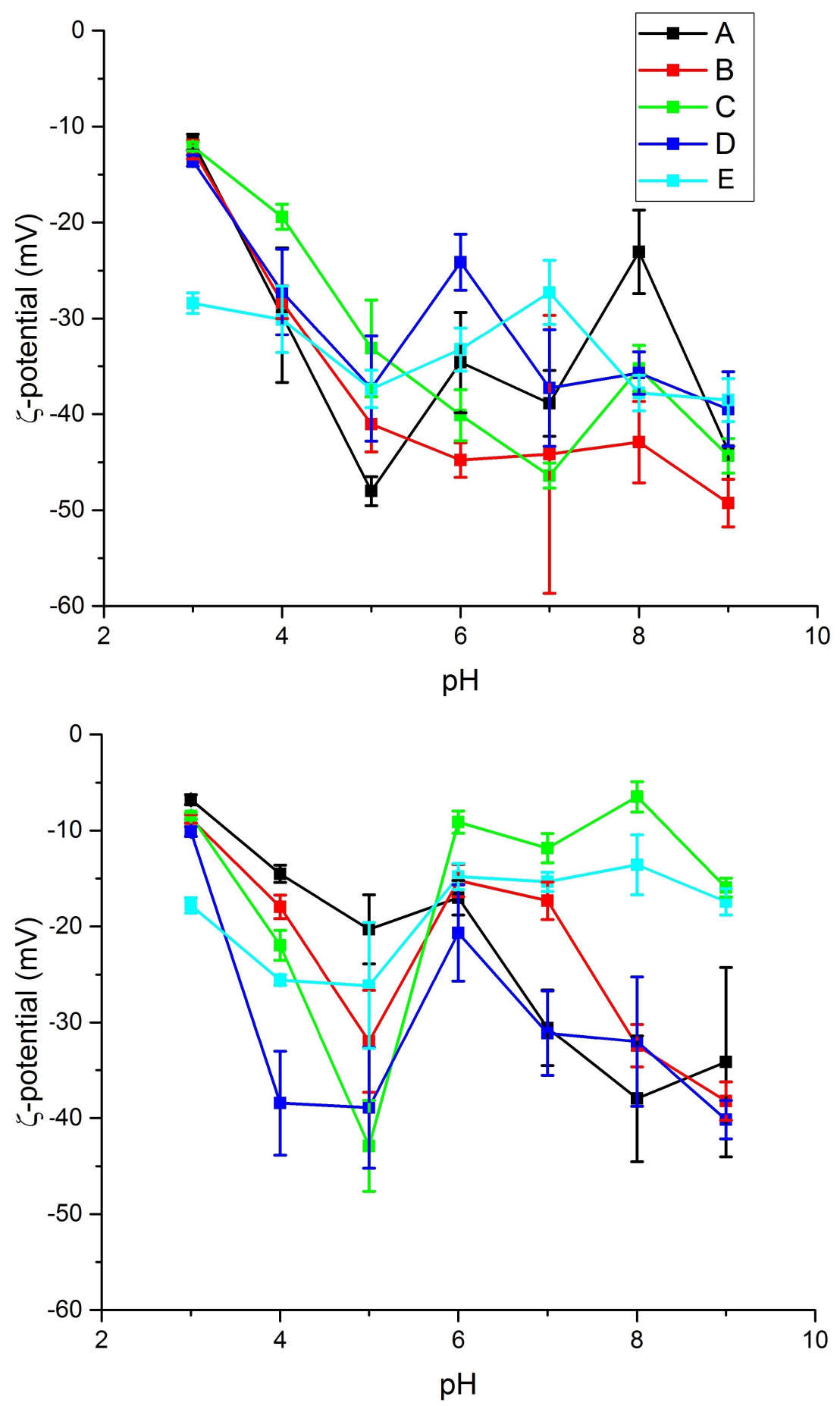

Figure 4.13: $\zeta$-potential of particle sets A-E at pH 3-9 using DLS for $100 \%$ PBS with (lower) and without (upper) $0.03 \% \mathrm{v} / \mathrm{v}$ Triton-X. 


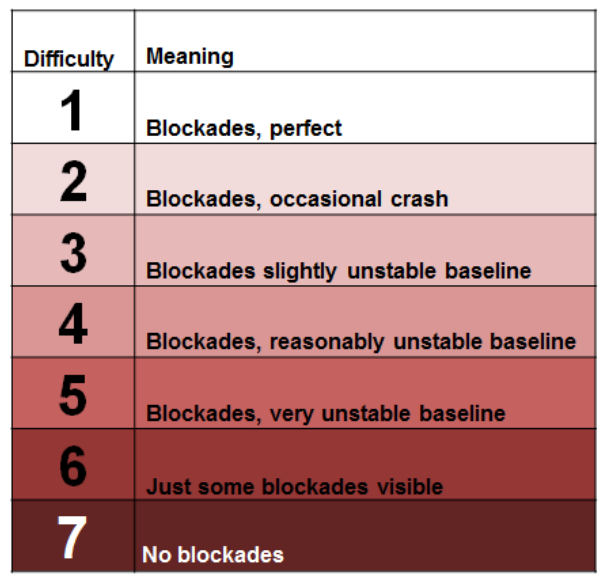

\begin{tabular}{|c|c|c|c|c|c|}
\hline & A & B & C & D & E \\
\hline pH 3 & 7 & 7 & 7 & 7 & 4 \\
\hline pH 4 & 6 & 6 & 6 & 4 & 3.5 \\
\hline pH 5 & 6 & 4 & 5 & 4 & 3.5 \\
\hline pH 6 & 4 & 4 & 4 & 2.5 & 4 \\
\hline pH 7 & 5 & 4 & 3 & 2.5 & 3.5 \\
\hline pH 8 & 4.5 & 3.5 & 3.5 & 2.5 & 3.5 \\
\hline pH 9 & 4 & 3.5 & 2 & 2 & 3.5 \\
\hline
\end{tabular}

\begin{tabular}{|l|l|l|l|l|l|}
\hline & A & B & C & D & E \\
\hline pH 3 & 6.5 & 7 & 7 & 7 & 3.5 \\
\hline pH 4 & 5 & 2 & 5 & 4 & 2 \\
\hline pH 5 & 2.5 & 2.5 & 4 & 1.5 & 2 \\
\hline pH 6 & 2.5 & 2.5 & 3 & 2 & 2 \\
\hline pH 7 & 1.5 & 1.5 & 2 & 1 & 2 \\
\hline pH 8 & 1.5 & 1 & 1.5 & 1.5 & 1.5 \\
\hline pH 9 & 1.5 & 1.5 & 2.5 & 1.5 & 2 \\
\hline
\end{tabular}

\section{Without surfactant}

\section{With surfactant}

Figure 4.14: Measurement difficulty scale (upper) with measurement difficulties for particle sets A-E in pH 3-9 with (right) and without (left) $0.03 \%$ $\mathrm{v} / \mathrm{v}$ Triton-X. 
work due to problems with particle stability without surfactant. Instead a scale was developed to rank measurement difficulty at different $\mathrm{pH}$ values. This scale is seen in Figure 4.14. For particles to be measurable it was thought that they needed to be a 3 or less on the scale.

Figure 4.14 also compares the difficulty of measurements made in 100 $\mathrm{mM} \mathrm{KCl}$ with and without surfactant. It can be seen that most particles were unmeasurable up to $\mathrm{pH} 9$ without surfactant. Particles became measurable at $\mathrm{pH} 5$ with surfactant. Particles became more stable the higher the $\mathrm{pH}$ and the higher their surface charge density, both with and without surfactant. CPN particles (set E), which are non-carboxylated, were mostly unaffected by the changes in $\mathrm{pH}$ but were still easier to measure with surfactant.

\subsection{Conclusion}

In this Chapter detailed theory and background of $\zeta$-potential and its measurement using TRPS were presented and discussed. Following this, a number of $\zeta$-potential measurement methods were outlined including a number of multi-point analysis methods in which the blockade was split 
into fractions and the electrokinetic component of each fraction was derived. The uncertainties associated with each method were described.

Method I and Method II were compared for charged and uncharged particles and it was found that Method II was superior discerning the particle types, regardless of pore geometry. Detailed error analysis of Method II found that blockade fractions closer to $n=0.3$ and measurements at higher voltages resulted in lower uncertainties. The large uncertainty in absolute values of $\zeta_{\text {particle }}$ for this method is due to Equation 4.11 and the combining of errors using Equation 4.12. This analysis was done without the errors associated with $\zeta_{C a l}$ and $\zeta_{\text {pore }}$.

Calibration $\zeta$-potential values were then obtained with DLS using two different DLS measurement cells, one of which was discovered to be of superior quality. This allowed for the measurement of $\zeta_{C a l}$ for a range of Izon Science calibration particles including those to be used in later measurements.

$\zeta$-potential measurements of nominally $200 \mathrm{~nm}$ particles each with a nominal surface charge were achieved in 3 different electrolytes using Method III, and in a single electrolyte at 11 concentrations using Method 
IV. For both DLS and TRPS there was no clear trend or difference observed between electrolytes. An increase in $\zeta_{\text {particle }}$ was seen with increasing electrolyte concentration as expected due to EDL compression at higher electrolyte concentrations. Trends with nominal particle surface charge were clearer with DLS than TRPS.

Detailed error analysis of Method IV was achieved, incorporating measured $\zeta_{C a l}$ and its related uncertainty. It was found that measurements at $n=0.2-0.45$ resulted in lower uncertainties. Lower uncertainties than Method II were observed due to less sources of random error. This analysis was done without errors associated with $\zeta_{\text {pore }}$ which would further improve this method. The large uncertainty in absolute values of $\zeta_{\text {particle }}$ for this method is due to incorporating $\zeta_{\mathrm{Cal}}$ and the combining of errors using Equation 4.12.

An initial investigation of $\mathrm{pH}$ variation was attempted. However, difficulties with surfactant effects on $\zeta$-potential trends were encountered using DLS. TRPS measurements of particles without surfactant were unsuccessful at all attempted $\mathrm{pHs}$ due to pore blocking. The issue of surfactant affecting $\zeta$-potential measurements and pore blocking (which is a commonly occurring problem in TRPS) could be addressed concurrently by 
applying non-fouling pore coatings. 


\section{Chapter 5}

\section{Improved $\zeta$-potential}

\section{Measurements}

In Chapter 4 , a number of $\zeta$-potential measurement methods were described and applied to particles in a range of conditions. In this Chapter, the final method (Method IV), is combined with streaming potential measurements taken using the qNano to give more complete $\zeta$-potential measurements.

First, streaming potential theory and the streaming potential measurements set-up are described. This is followed by investigations into com- 
bined $\zeta_{\text {pore }}$ and $\zeta_{\text {particle }}$ measurements at different stretches, electrolyte concentrations and pHs. Finally, a number of pore coating methods, including dopamine coating, polymer brush functionalisation and layer-by-layer polyelelectrolyte coating, are investigated using $\zeta_{\text {pore }}$ and ion-current rectification (ICR) measurements.

\subsection{Streaming Potential Theory}

When an electrolyte solution is forced to flow through a capillary with charged walls (e.g. a nano or micro-pore) by hydraulic pressure the charges in the mobile part of the double layer near the wall are carried downstream. This charge flow gives rise to a streaming current $I_{s}$ and the accumulation of charge at the ends of the capillary generates a potential difference and hence an electric field. This field causes a conduction current in the opposite direction $\left(I_{c}\right)$. When these two currents are equal $\left(I_{s}=I_{c}\right)$ a steady state is achieved and the resulting electrostatic potential difference between the ends of the channel is the streaming potential $(\Delta \Phi)$. The streaming potential is related to the $\zeta$-potential by the HelmholtzSmoluchowski equation [221]; 


$$
\frac{\Delta \Phi}{\Delta P}=\frac{\varepsilon \zeta}{\eta \lambda}
$$

where $\Delta \Phi$ is the streaming potential, $\Delta P$ is the pressure drop and $\varepsilon, \eta$ and $\lambda$ are the permittivity, viscosity and conductivity of the electrolyte respectively. Streaming potential measurements using Equation 5.1 must take place in the thin EDL limit [223]. Otherwise, geometry-dependent corrections are necessary [226-228]. Highly conductive channel materials also require corrections [229].

Streaming potential is not commonly used in the characterisation of RPS pores but is very commonly used to characterise the surface charge of porous membranes [230]. Some of the few examples of RPS pores characterised using streaming potential include carbon nanotube-based Coulter Counters [58, 59, 231], PDMS micro-channels [232], and TPU pores [46, 47, 111]. Modelling of streaming potential in single channels has also been investigated [233]. 


\subsection{Streaming Potential Measurement Set-Up}

The TRPS streaming potential measurement set-up used here was inspired by the work of Anderson et al. [234]. The set-up requires a qNano, a syringe (and optional syringe pump), a multimeter, a pressure sensor, and a computer as well as the relevant connectors and tubing (Figure 5.1).

Some slight, reversible modifications to the $q$ Nano are required to measure streaming potential. The back plate should be removed so that an SMA connector can be fed from the lower fluid cell to the multimeter while keeping most of the electronics shielded within the qNano body. It is also useful to removed the baseplate of the qNano for ease of access to the lower electronics if switching between measurement modes (e.g. Streaming potential vs. classical TRPS).

The syringe can be placed in a syringe pump or operated by hand. It can be connected to the upper fluid cell using the same tubing that is part of the VPM (which can be easily removed from the VPM). A good quality multimeter with $0.001 \mathrm{mV}$ resolution is required. The one used here is a Keysight Technologies U1272A Digital Multimeter. The pressure sensor used in the present research is a circuit board containing a differential gas 


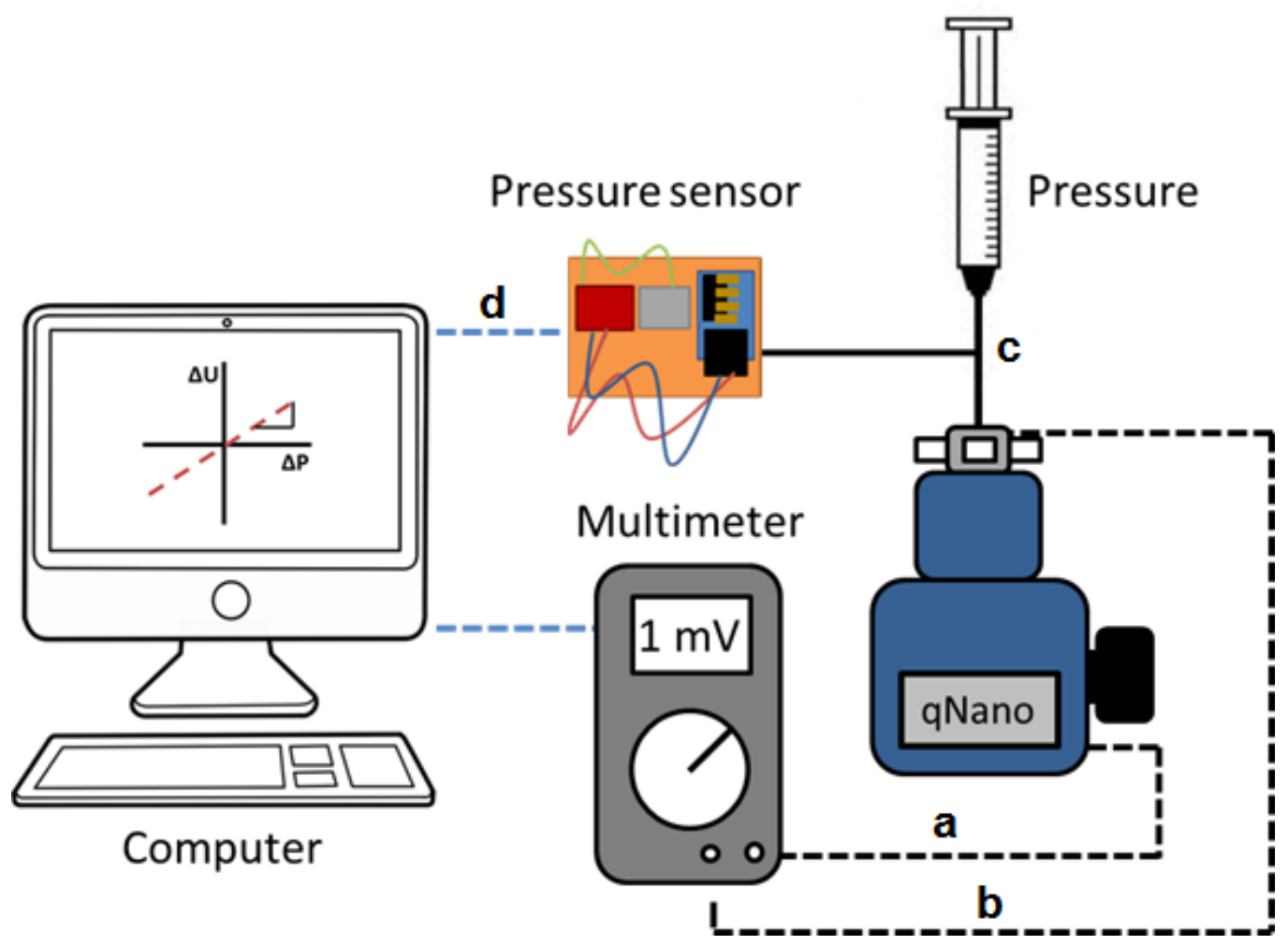

Figure 5.1: Schematic of TRPS streaming potential measurement set-up showing computer, multimeter, pressure sensor, pressure application device and qNano. Connectors and tubing are labelled a-d with (a) the SMA connector rewired from the qNano to the multimeter using a BNC to female double banana plug adapter and a BNC to SMA adapter, (b) alligator clip to banana plug cable, (c) tubing to connect the syringe to the top of the qNano and to the pressure sensor which includes a three-way splice, and (d) a micro USB to USB cable to connect the pressure sensor to the computer. 
pressure sensor $( \pm 50 \mathrm{kPa}$, Phidgets $)$, connected to an analog-digital converter (MCP3424 4-Channel 18-bit, DFRobot) and an Arduino compatible microcontroller (Pro Micro 5V/16MHz, Sparkfun Electronics). The computer must have Arduino software installed to log the pressure read outs.

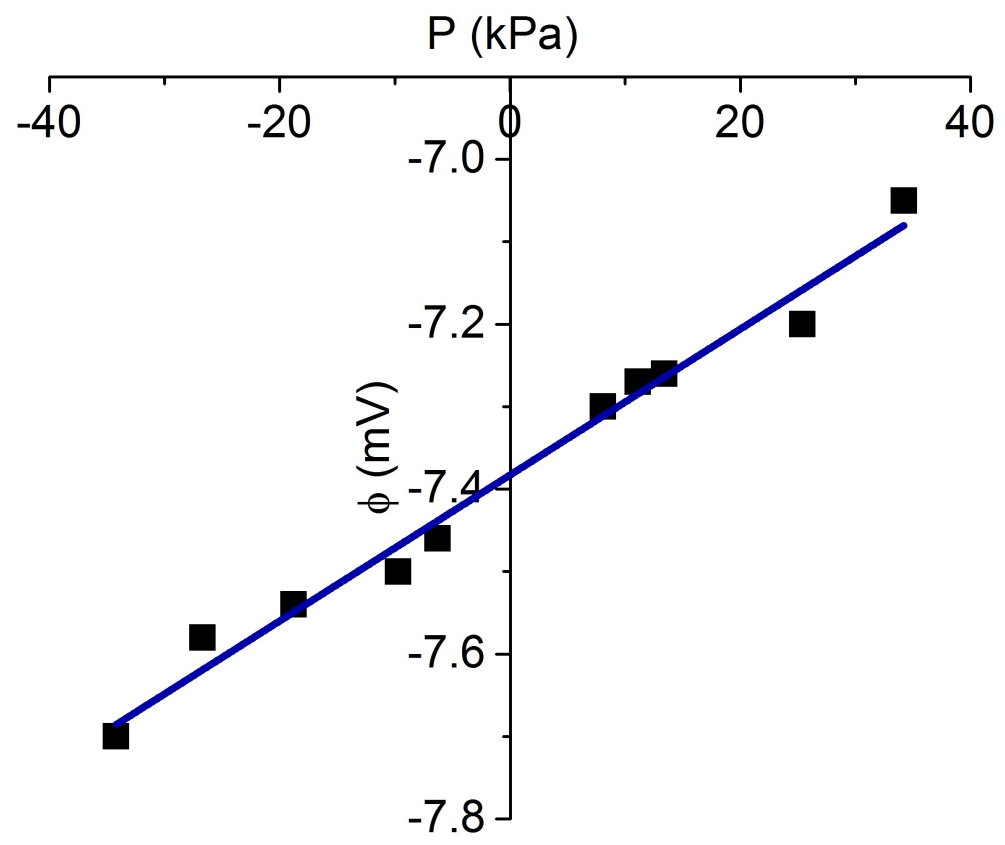

Figure 5.2: Example of streaming potential vs. pressure plot including the linear fit used to calculate $\zeta_{\text {pore }}$.

Pores were soaked in the chosen electrolyte in the qNano set up for 10-30 minutes before measurement. To take a $\zeta_{\text {pore }}$ measurement the upper and lower fluid cells were filled with electrolyte and then step wise 
pressure variation of between $-30 \mathrm{kPa}$ and $30 \mathrm{kPa}$ was applied with approximately 5 - $10 \mathrm{kPa}$ increments applied and the resulting streaming potential was read off the multimeter. A pressure vs. voltage plot is obtained (Figure 5.2) and $\zeta_{\text {pore }}$ is calculated from the slope by applying the Helmholtz-Smoluchowski equation (Equation 5.1).

\section{3 $\zeta_{\text {particle }}$ and $\zeta_{\text {pore }}$ Measurements}

\subsubsection{Pore Size}

In this Section, the effect of pore diameter on pore charge was investigated. It is expected that $\zeta_{\text {pore }}$ is independent of pore size and is determined only by the pore material and the electrolyte as per Equation 5.1.

Figure 5.3 shows measured $\zeta_{\text {pore }}$ with stretch for 3 different pore size ratings in 2 different electrolyte concentrations. It can be seen that at the higher electrolyte concentration (100\% PBS) there is little variation in measured $\zeta_{\text {pore }}$ seen over the stretch range. At the lower electrolyte concentration (10\% PBS) at lower stretches a less negative $\zeta$-potential is measured which appears to equilibrate to a steady $\zeta_{\text {pore }}$ at higher stretches. Low elec- 


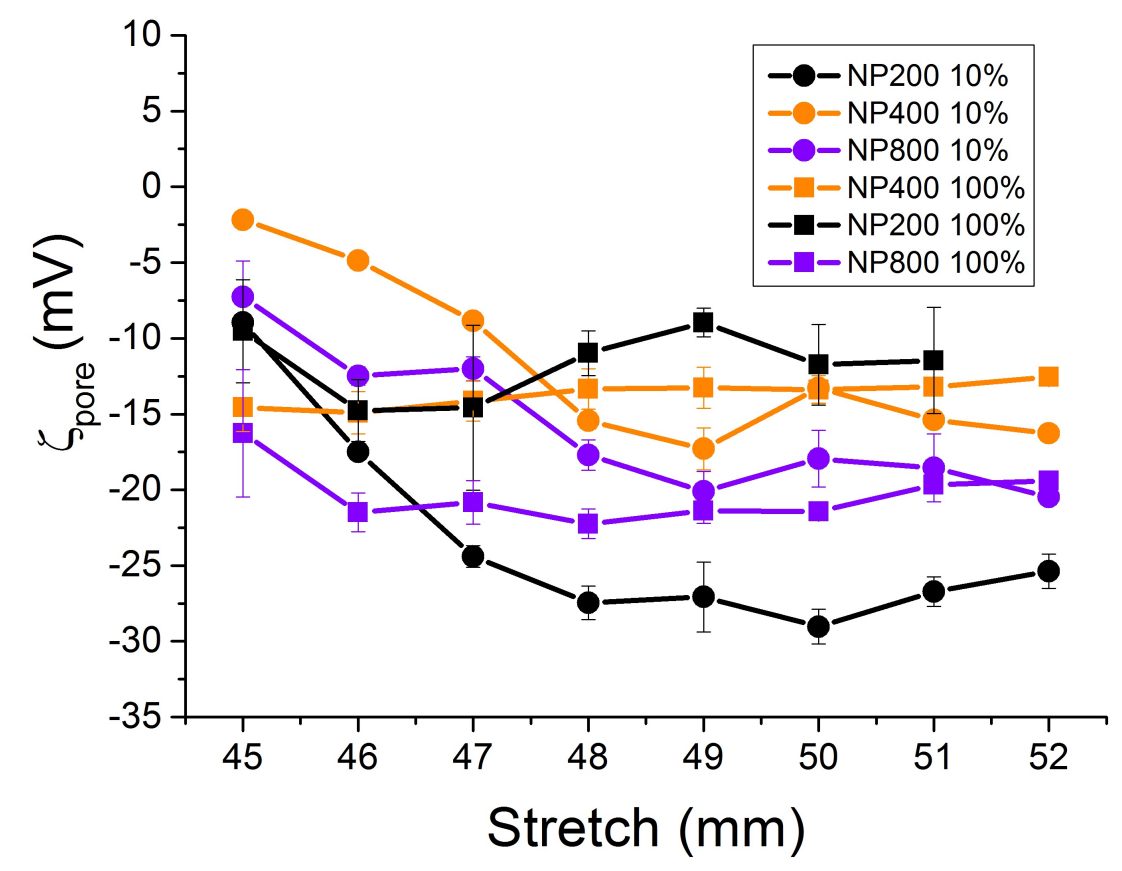

Figure 5.3: $\zeta_{\text {pore }}$ measurements with stretch for 3 different pore ratings (NP800, NP400 and NP200) and two different electrolyte concentrations (10\% and $100 \%$ PBS). Data points and error bars represent the average and standard deviation respectively over 5 measurements. Lines connecting average data points are included to highlight the observed trend. 
trolyte concentrations have introduced uncertainty into previous streaming potential measurements [223]. Alternatively, lower salt concentrations can prevent a pore from wetting fully [235] and these results could suggest that pore wetting increased with increasing pore size until the pore was fully wet and consistent $\zeta_{\text {pore }}$ measurements can be achieved across a range of stretches. Concentration distribution effects have been seen previously at low salt concentrations in relatively small pores [236].

Once these variations in measured $\zeta_{\text {pore }}$ with stretch were understood it was possible to make measurements of $\zeta_{\text {particle }}$ which used a measurement of $\zeta_{\text {pore }}$ made immediately prior using the $q N a n o$ and the same experimental conditions. Figure 5.4 shows $\zeta_{\text {pore }}$ and $\zeta_{\text {particle }}$ measurements of two different particle sets. A number of approaches were used to obtain the results, including measurements at constant voltage and constant current, and averaging $\zeta_{\text {pore }}$ over a range of stretches.

It can be seen that $\zeta_{\text {particle }}$ is always more negative for particle set $\mathrm{H}$ than for set $\mathrm{E}$. This is as expected as particle set $\mathrm{H}$ are carboxylated polystyrene particles with a surface charge density of $159 \mu \mathrm{eq} / \mathrm{g}$ and particle set $\mathrm{E}$ are non-carboxylated polystyrene particles. Another observation is that the $\zeta_{\text {particle }}$ values of the two particle sets seem to converge at higher stretch 

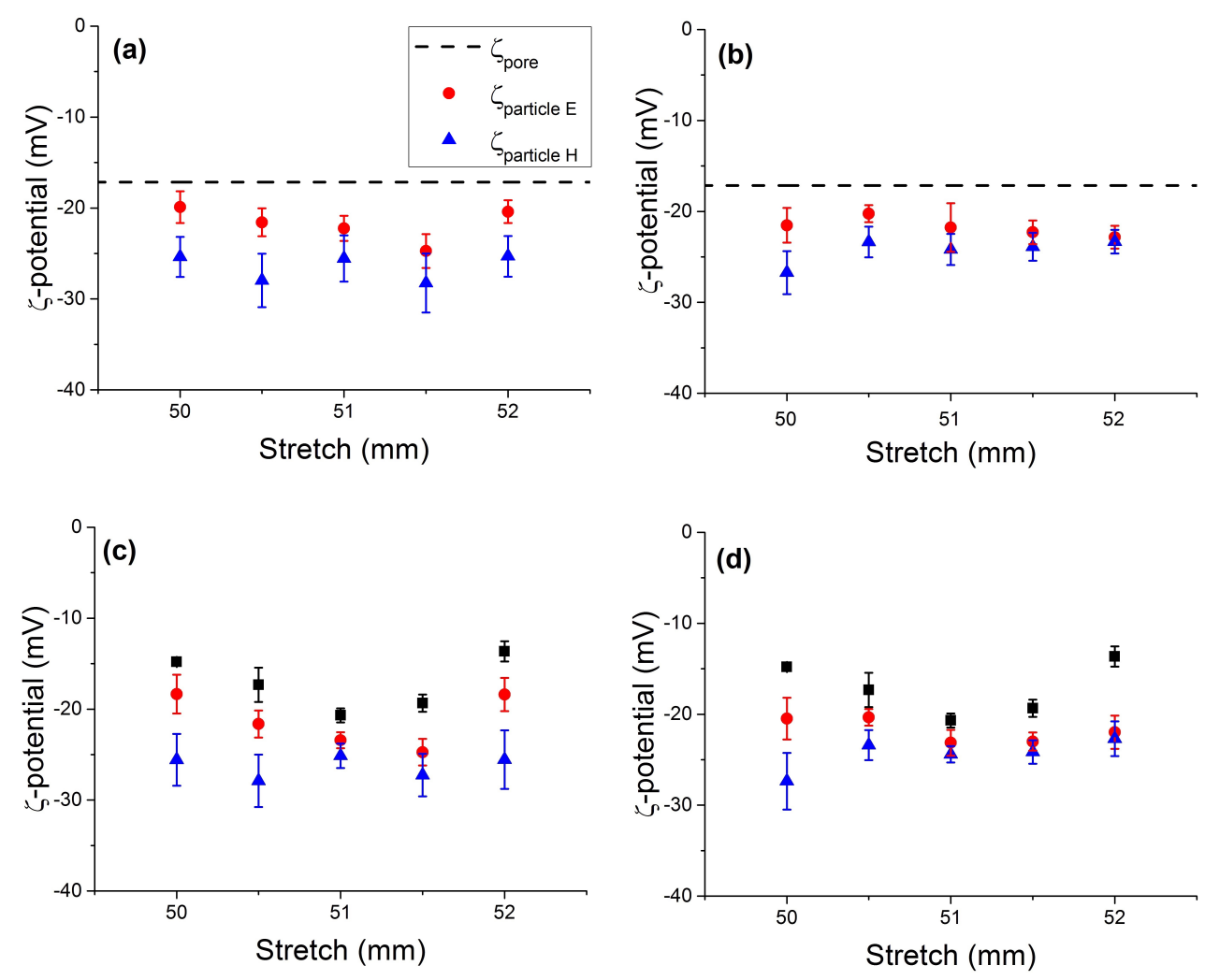

Figure 5.4: $\zeta_{\text {pore }}$ and $\zeta_{\text {particle }}$ measurements for two different particle sets (E and $\mathrm{H}$ Table 3.3) at 5 different stretches. (a) and (c) are measured at constant voltage, (b) and (d) are measured at constant current. For (a) and (b) $\zeta_{\text {pore }}$ was averaged over the 5 stretches and is represented by a dashed line whereas for $(\mathrm{c})$ and $(\mathrm{d})$ the measured $\zeta_{\text {pore }}$ at each stretch is shown as data points. Data points and error bars represent the average and standard deviation respectively over 3 measurements. 
for measurements at constant current (Figure 5.4b and d). This is not seen to such an extent for measurements at constant voltage. As stretch is increased, the baseline current increases and the blockade magnitude decreases. The latter causes a loss of resolution for the multi-point charge analysis. Decreasing the voltage with increased stretch to achieve a constant current means that this effect is exaggerated because blockade magnitude also decreases with decreasing applied voltage and hence particle sets cannot be distinguished at higher stretches. Averaging $\zeta_{\text {pore }}$ across the stretches removes random variation from this source across stretches.

\subsubsection{Electrolyte Concentration}

This Section features measurements of $\zeta_{\text {particle }}$ and $\zeta_{\text {pore }}$ at different electrolyte concentrations. These measurements build on measurements in Section 4.2.3. New particle sets with varying surface charge densities, obtained from the same manufacturer (Bangs Labs), were used in these experiments due to stock availability. These particle sets were also manufactured under near identical conditions so it was expected that their surface charge densities provided by the manufacturer could be more directly compared between particle samples. Particle sets B and E-L were used, de- 
tails can be found in Table 3.3.

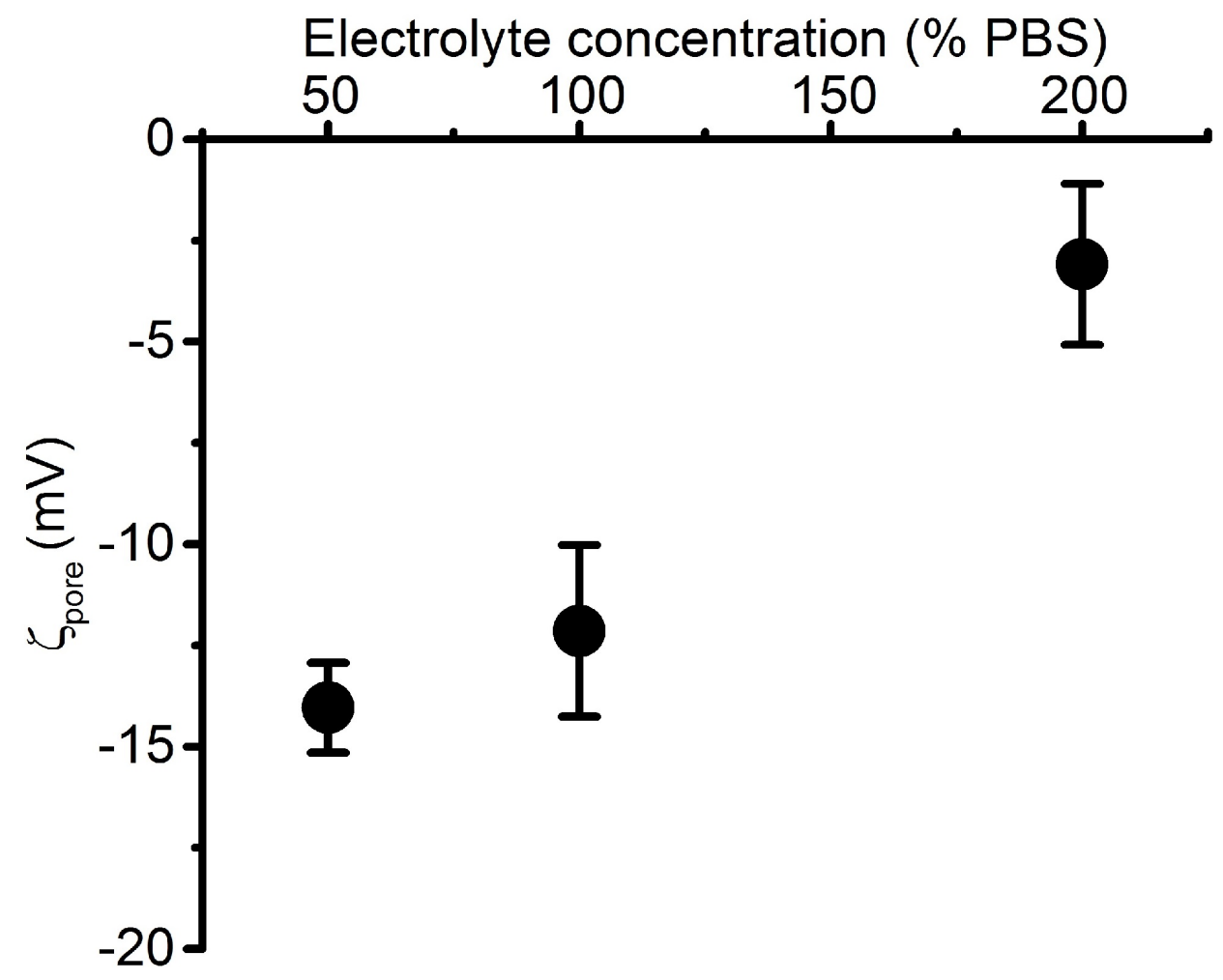

Figure 5.5: $\zeta_{\text {pore }}$ for an uncoated NP150 pore where $X=52 \mathrm{~mm}$ at 3 electrolyte concentrations. These were measured using the qNano streaming potential set-up. Error bars represent the standard deviation across 5 measurements.

The same electrolyte concentration experiments undertaken in Chapter 4 were also undertaken here with the addition of the TRPS streaming potential measurements. Due to this extra level of complexity, and the increase in the number of particle sets examined, only 3 electrolyte con- 
centrations were examined: 50\%, 100\% and 200\% PBS. DLS was also used to measure $\zeta_{\text {particle }}$ as a comparison.

As explained previously, an increase (i.e. becomes less negative) in $\zeta_{\text {particle }}$ and $\zeta_{\text {pore }}$ is expected with increasing electrolyte concentration. It is also expected that particle charge follows the same trend as the surface charge density values given by the manufacturer. An increase in $\zeta_{\text {pore }}$ with increasing electrolyte concentration was observed (Figure 5.5). However, there was no clear increase in $\zeta_{\text {particle }}$ with electrolyte concentration especially in the DLS measurements (Figure 5.6). In the TRPS measurements the relationship between measured $\zeta_{\text {particle }}$ at 50\% PBS and 100\% PBS was as expected for particle sets F-K (Table 3.3). The $\zeta$-potentials measured in 200\% PBS were more negative than expected. Most surprisingly no correlation was seen between the manufacturer's surface charge density assignments and measured $\zeta_{\text {particle }}$ using either DLS or TRPS, which brings into question the validity of comparing these surface charge density values to mobility measurements.

Compared to Section 4.2.3, less of a trend is seen between electrolyte concentrations and between particle types with both DLS and TRPS. This may be because fewer electrolyte concentrations were examined ( 3 vs. 11 

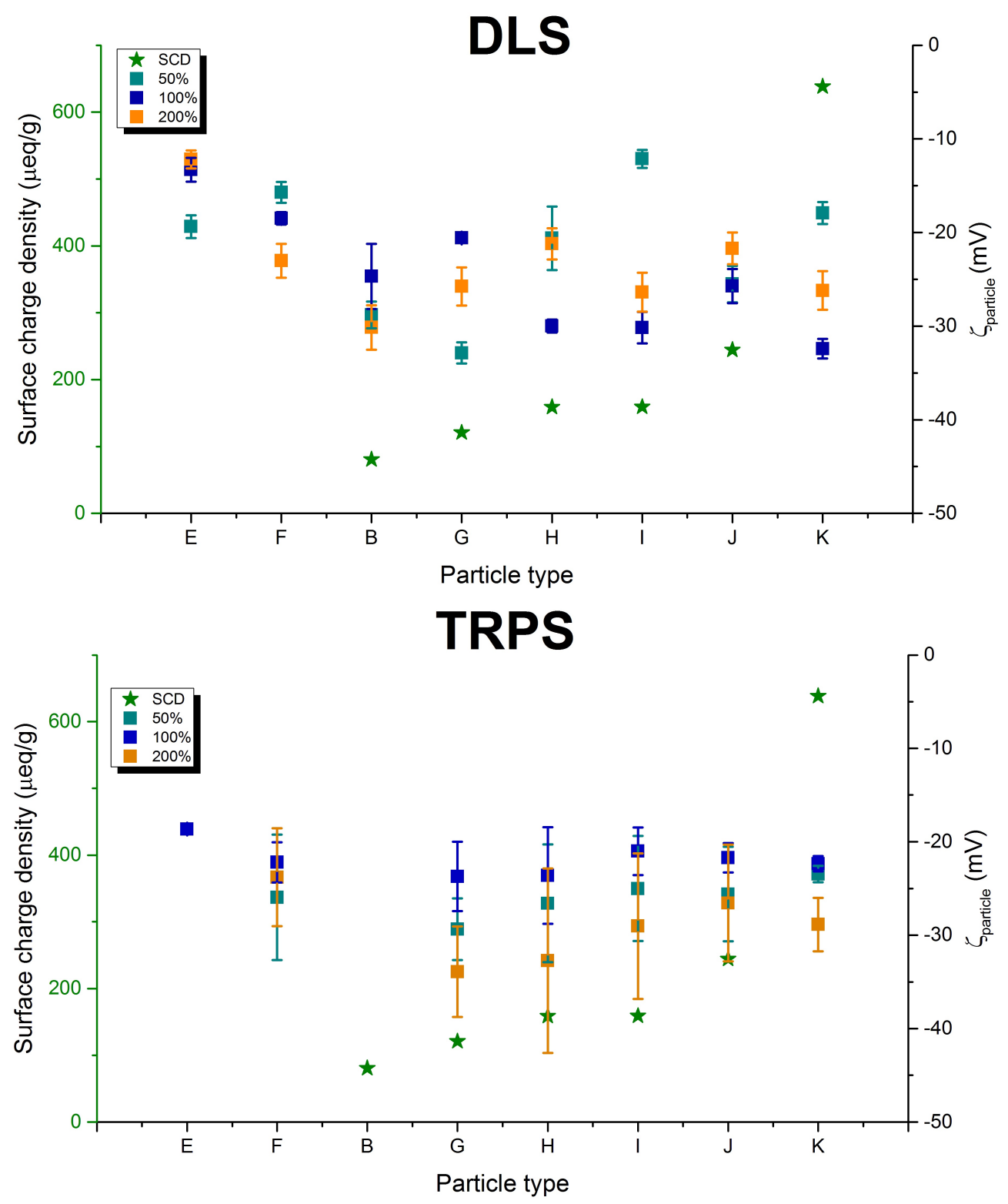

Figure 5.6: DLS and TRPS $\zeta_{\text {particle }}$ measurement of 8 particle types in 3 different PBS concentrations. Details of particle sets can be found in Table 3.3. Dark green stars show the surface charge density of the particles as specified by the manufacturer. Data points and error bars represent the average and standard deviation over 10-15 repeat measurements for DLS and 3 repeat measurements for TRPS. 
in Chapter 4). The lack of trend with particle surface charge density is present for both DLS and TRPS suggesting that this may be an issue with the particles or the direct comparison of surface charge density to mobility. Particle set B was used as calibration particles. The charge on particle set E was too lowly charged to be measured using Method IV.

\section{Uncertainties}

Uncertainty calculations were conducted for Method IV with measured $\zeta_{\text {pore }}$ to provide an example of the magnitude of uncertainties in absolute values of $\zeta_{\text {particle }}$ and where they are introduced. The random error in $\zeta_{\text {Sample }}$ measurements using Method IV for particle sets F-K in $50 \%$ PBS was calculated. These calculations included the errors associated with $\zeta_{C a l}$ and $\zeta_{\text {pore }}$. Combining the uncertainties in $v_{n S a m p l e}^{e l}, v_{n C a l}^{e l}, \zeta_{C a l}$, and $\zeta_{\text {pore }}$ using Equation 4.12 gives the percentage uncertainty in $\zeta_{n \text { Sample }}$. These uncertainties at each $n$ for the 6 particle sets can be found in Table 5.1. It can be seen that the error in $\zeta_{n \text { Sample }}$ is lowest at values of $n$ between 0.20.45 , but it is somewhat particle dependant. Again, when $n$ is larger $I_{n}$ is closer to the baseline current and therefore more error is introduced. It can be seen that compared to the random errors presented in Section 4.2.3 
the percentage errors are larger, probably due to the included variation in measured $\zeta_{\text {pore }}$ using Equation 4.12.

Table 5.1: Random errors in $\zeta_{n \text { Sample }}$ for particles F-K in $50 \%$ PBS using Method IV.

\begin{tabular}{|c|c|c|c|c|c|c|}
\hline$n$ & $\mathrm{~F}$ & $\mathrm{G}$ & $\mathrm{H}$ & $\mathrm{I}$ & $\mathrm{J}$ & $\mathrm{K}$ \\
\hline 0.05 & $36.1 \%$ & $45.0 \%$ & $55.8 \%$ & $31.7 \%$ & $59.2 \%$ & $43.1 \%$ \\
\hline 0.1 & $35.5 \%$ & $41.0 \%$ & $55.6 \%$ & $29.6 \%$ & $57.6 \%$ & $42.4 \%$ \\
\hline 0.15 & $34.8 \%$ & $40.0 \%$ & $56.4 \%$ & $28.7 \%$ & $55.6 \%$ & $41.6 \%$ \\
\hline 0.2 & $35.2 \%$ & $40.1 \%$ & $56.9 \%$ & $28.3 \%$ & $55.2 \%$ & $41.3 \%$ \\
\hline 0.25 & $35.7 \%$ & $41.6 \%$ & $58.0 \%$ & $29.1 \%$ & $55.3 \%$ & $41.7 \%$ \\
\hline 0.3 & $36.8 \%$ & $42.8 \%$ & $59.6 \%$ & $29.9 \%$ & $56.1 \%$ & $42.3 \%$ \\
\hline 0.35 & $37.6 \%$ & $44.2 \%$ & $60.5 \%$ & $30.5 \%$ & $56.5 \%$ & $43.0 \%$ \\
\hline 0.4 & $38.3 \%$ & $44.1 \%$ & $60.9 \%$ & $32.1 \%$ & $57.4 \%$ & $43.8 \%$ \\
\hline 0.45 & $39.1 \%$ & $44.8 \%$ & $61.4 \%$ & $33.8 \%$ & $58.0 \%$ & $44.4 \%$ \\
\hline 0.5 & $39.6 \%$ & $46.3 \%$ & $61.8 \%$ & $35.0 \%$ & $58.4 \%$ & $45.1 \%$ \\
\hline 0.6 & $39.4 \%$ & $51.4 \%$ & $61.5 \%$ & $37.2 \%$ & $58.3 \%$ & $45.2 \%$ \\
\hline 0.7 & $38.6 \%$ & $57.6 \%$ & $60.3 \%$ & $38.2 \%$ & $57.5 \%$ & $44.3 \%$ \\
\hline 0.8 & $37.3 \%$ & $60.4 \%$ & $57.5 \%$ & $38.8 \%$ & $56.6 \%$ & $42.9 \%$ \\
\hline 0.9 & $40.8 \%$ & $63.1 \%$ & $57.1 \%$ & $42.9 \%$ & $56.8 \%$ & $42.4 \%$ \\
\hline
\end{tabular}

To calculate the average $\zeta$-potential values for the sample $\zeta_{\text {Sample }}$ must be averaged over $n$. The values of $n$ to be averaged over can be chosen based on the lowest observed uncertainties in previous calculations. When averaged over $n=0.2-0.45$ for particle sets $F, G, H, I, J$ and $\mathrm{K}$ there is a 2.4, $5.0,4.3,3.0,2.6$, and $2.1 \%$ error respectively in $\zeta_{\text {Sample }}$.

To summarise, for absolute values of $\zeta_{\text {particle }}$ there can be large errors (Table 5.1), the magnitude of these errors is due to calculations using Equation 4.12 which now include uncertainty in $\zeta_{\text {Cal }}$ and $\zeta_{\text {pore }}$. These uncer- 
tainty calculations have the least unknown systematic uncertainty because $\zeta_{C a l}$ and $\zeta_{\text {pore }}$ have been measured. This is the main error as it is much larger than the uncertainty due to averaging across $n$.

Data points and error bars for TRPS in Figure 5.6 are the average and standard deviation over minimum 500 particles. These error bars therefore represent the random error in $\zeta_{\text {Sample }}$ without the inclusion of the other random errors outlined in Table 4.1.

\subsection{3 $\mathrm{pH}$}

The isoelectric point of TPU pores was investigated in $100 \mathrm{mM} \mathrm{KCl} \mathrm{us-}$ ing streaming potential measurements. It is important to know how pore charge changes with $\mathrm{pH}$ when designing experiments because the relationship between pore and particle charge will affect ease of use of the technology. Figure 5.7 shows $\zeta_{\text {pore }}$ change with $\mathrm{pH}$ measured using the qNano streaming potential set-up. The isoelectric point was found to be between $\mathrm{pH} 2$ and $\mathrm{pH} 3$, with linear interpolation between these points giving a $\mathrm{pH}$ of 2.35 , which is similar to values found in the literature [237].

The charge of a range of different particle sets was measured at dif- 


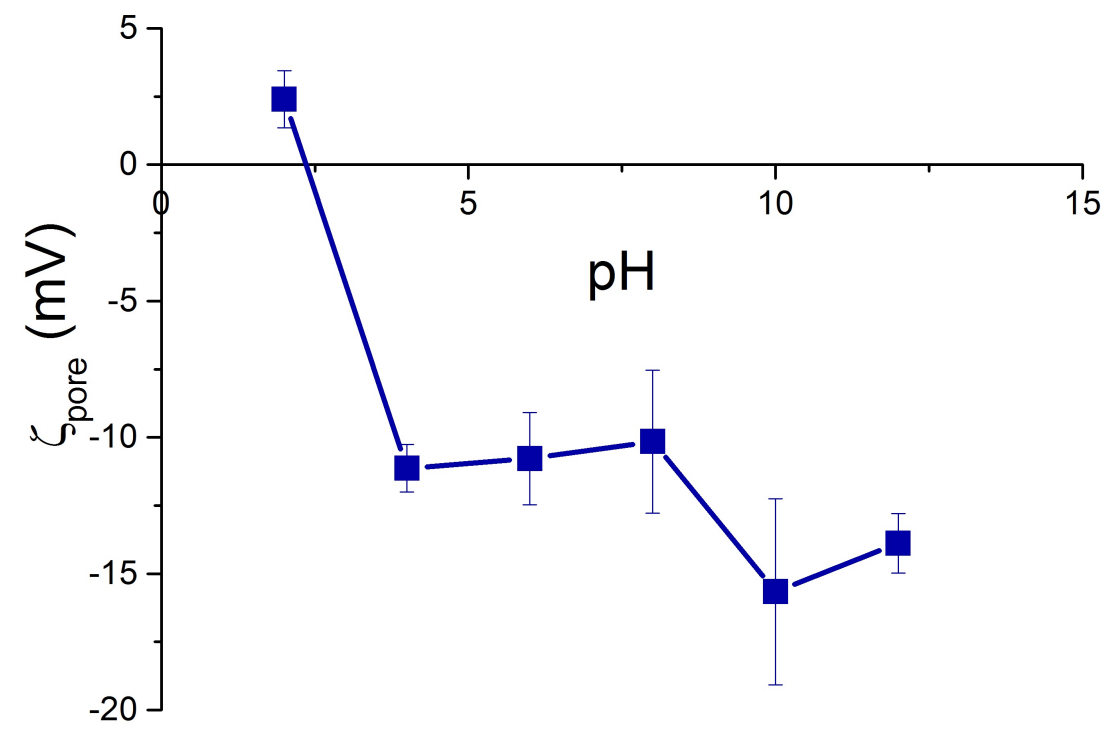

Figure 5.7: $\zeta_{\text {pore }}$ measurement using TRPS at a range of $\mathrm{pHs}$ to identify the isoelectric point of the pore material in $\mathrm{KCl}$. The isoelectric point was found to be between $\mathrm{pH} 2$ and $\mathrm{pH} 3$ and can be estimated as 2.35 using linear interpolation between these points. Data points and errors bars are the average and standard deviation over 3 measurements respectively. 

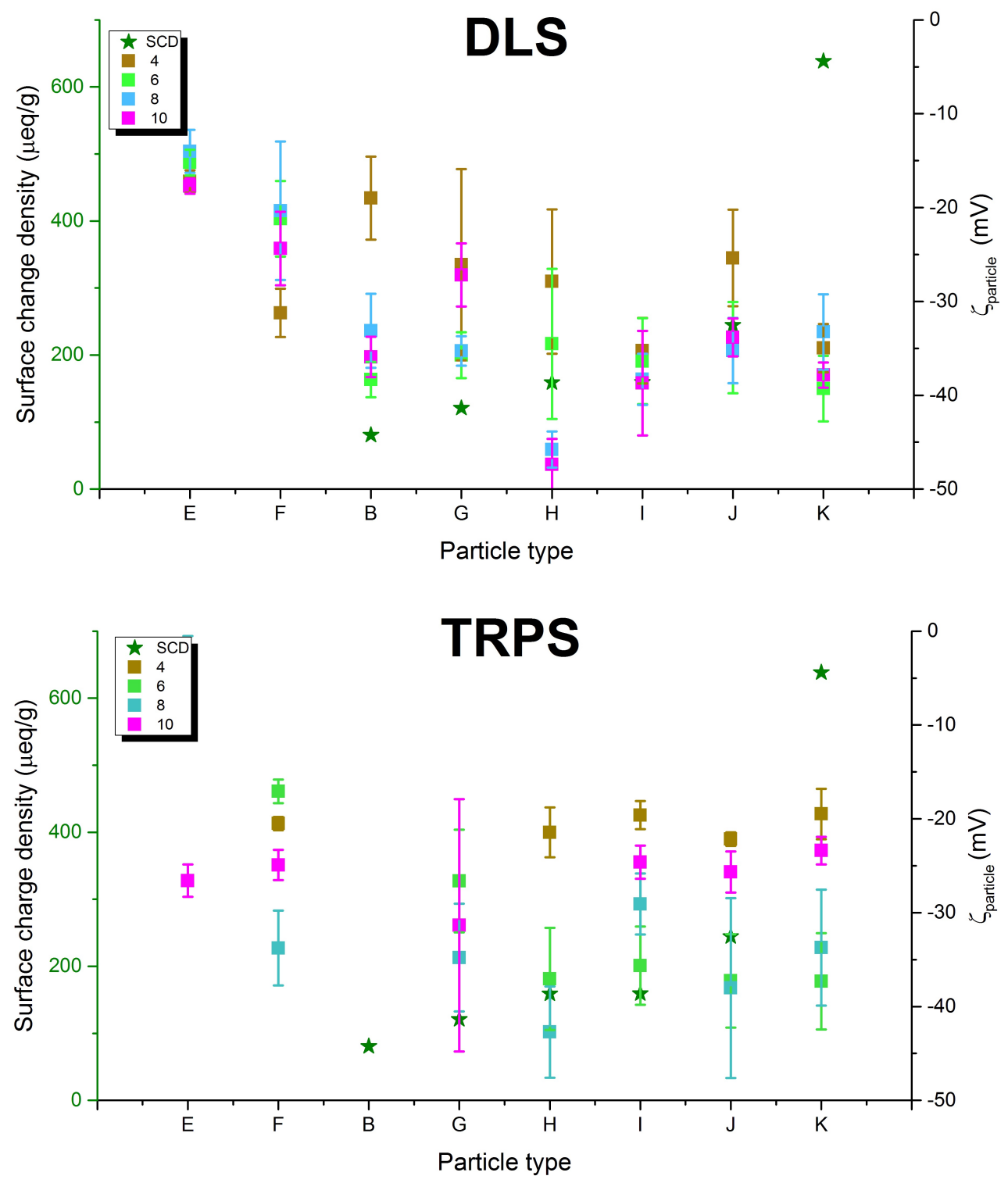

Figure 5.8: DLS and TRPS $\zeta_{\text {particle }}$ measurement of 8 particle types at 4 different pHs. Details of particle sets can be found in Table 3.3. Dark green squares show the surface charge density of the particles as specified by the manufacturer. 
ferent pHs. Surfactant was added to the solutions to prevent aggregation and pore clogging and therefore make the particles measurable. Figure 5.8 shows TRPS and DLS $\zeta_{\text {particle }}$ measurement of 8 particle types (Table 3.3) at 4 different $\mathrm{pHs}$. It is expected that as the $\mathrm{pH}$ becomes more acidic the charge on the particles will become less negative (as with the pore). Unfortunately, no concrete trends were seen with either TRPS or DLS. For the TRPS data, pH 10 and pH 6 data generally do not fit the expected trends. The $\mathrm{pH} 8$ and $\mathrm{pH} 4$ measurements mostly fit the expected trend when compared to each other. There was also no trend seen with between particle surface charge density and measured $\zeta_{\text {particle. }}$. As surfactant was added to all these samples it is possible that this addition shields the surface charge and disrupts expected trends. Similarly to Figure 5.6 it may also be due to the particles or the direct comparison of surface charge density to mobility.

In an attempt to move away from using surfactant so charge trends could be better examined, pore coatings which may prevent pore clogging were investigated. 


\subsection{Coatings}

A regularly occurring problem amongst TRPS users is blocking of the pore, particularly by biological particles [163]. Surfactant is often added to help prevent these blockages. Surface coatings that alter $\zeta_{\text {pore }}$ and may remove the need for surfactant so that more accurate $\zeta$-potential values can be measured are investigated here. Efforts to control the surface properties of single nanopores and nanoporous materials have been reported. These include deposition of metals and metal oxides [238], modification with organic molecules [239, 240], DNA [241], and polymer brushes [242].

In this Section, three coating types are examined. Dopamine coatings, layer-by-layer polymer coatings, and polymer brush coatings were used. Changes in the pore were monitored by XPS and FTIR (for dopamine coatings), $I-V$ curves, and streaming potential measurements. No particles were able to be measured on any of the coated pores. It is unclear whether this is because of decreased pore size increased pore charge or a combination. 


\subsubsection{Dopamine}

Dopamine is most commonly recognized as a neurotransmitter which plays a role in pleasure and motivation [243]. However, recently dopamine has been utilised as a multifunctional surface coating [244-246]. Dopamine mimics the non-discriminatory adhesiveness of biological melanins such as L-DOPA. L-DOPA is one of the glue like proteins that marine mussels use to attach to surfaces. Mussels are able to attach to a wide range of organic and inorganic surfaces in aqueous environments where most adhesives function sub-standardly. Dopamine, in a $\mathrm{pH}$ similar to marine environments ( $\mathrm{pH} 8.5)$, can be used to simply dip coat the material of choice, as a thin film of polydopamine is formed [245].

Dopamine has been used to coat a range of surfaces including gold, silver, glass and polymers such as PDMS and polyurethane [245]. Polydopamine, and polydopamine coated substrates have potential applications in energy, biomedicine, sensing, and water treatment [247]. Most of these applications result from the stickiness of polydopamine. Its catechol, amine and imine functional groups, which allow it to polymerise in the first place, also make it attractive to a range of other chemicals, and chem- 
ical functionalities, as well as cells and nanoparticles [247]. In biomedical applications, whereas quite often the research focus is for a non-fouling surface, polydopamine can be used to capture fungi or bacteria for analysis, due to its adhesive nature [248]. In coatings, polydopamine is often used as a primary coating, which is followed by a secondary coating containing the desired functionality [245]. Common secondary coatings include metal films, electrolessly plated using the catechol-metal interaction, and organic second layers using interactions of other functional groups such as thiols and amines with the catechol group [245]. Generally, the addition of these ad-layers only requires a simple dip coat, in suitable conditions, as with the initial polydopamine coating.

Easily controlling the surface properties of the tunable pores used in TRPS is of great interest as it would provide more insight into the processes going on within the pore during measurements as well as making the pores easier to use when measuring a wider range of particles. Only one instance of polydopamine coated pores is reported in the literature. The pores however were not intended for use with particles. Pérez-Mitta et al. [240] modified "bullet" shaped poly(ethyleneterephthalate) pores with polydopamine and with a secondary coating of 3-aminobenzylamine 
(ABA). Polydopamine resulted in a negatively charged pore surface and ABA switched this to a positively charged surface. The charge on the surface was monitored using $I-V$ curves and the degree of current rectification observed. Gold was also used as a secondary coating to the dopamine, which was then modified with a tertiary coating using thiols. This was again monitored using $I-V$ curves, and a negative, and then positive charge pattern was observed respectively.

Due to the simplicity of the coating method, polydopamine was used as a first step in coating TPU tunable pores in this Thesis. To examine whether the coating was successful FTIR, XPS, $I-V$ curves and $\zeta_{\text {pore }}$ measurements were used. It has also been shown that metal ions co-ordinate to catechols such as dopamine which could result in more even, more complete coating [249]. $\mathrm{FeCl}_{2}$ was added to some pore coating mixtures (dopamine $\left.\left(\mathrm{Fe}^{2+}\right)\right)$ to see if a more complete coating could be achieved.

\section{FTIR}

FTIR spectra are taken in transmittance mode and correspond to unmodified TPU, polydopamine coated TPU (12 hour coating) and dopamine $\left(\mathrm{Fe}^{2+}\right)$ 


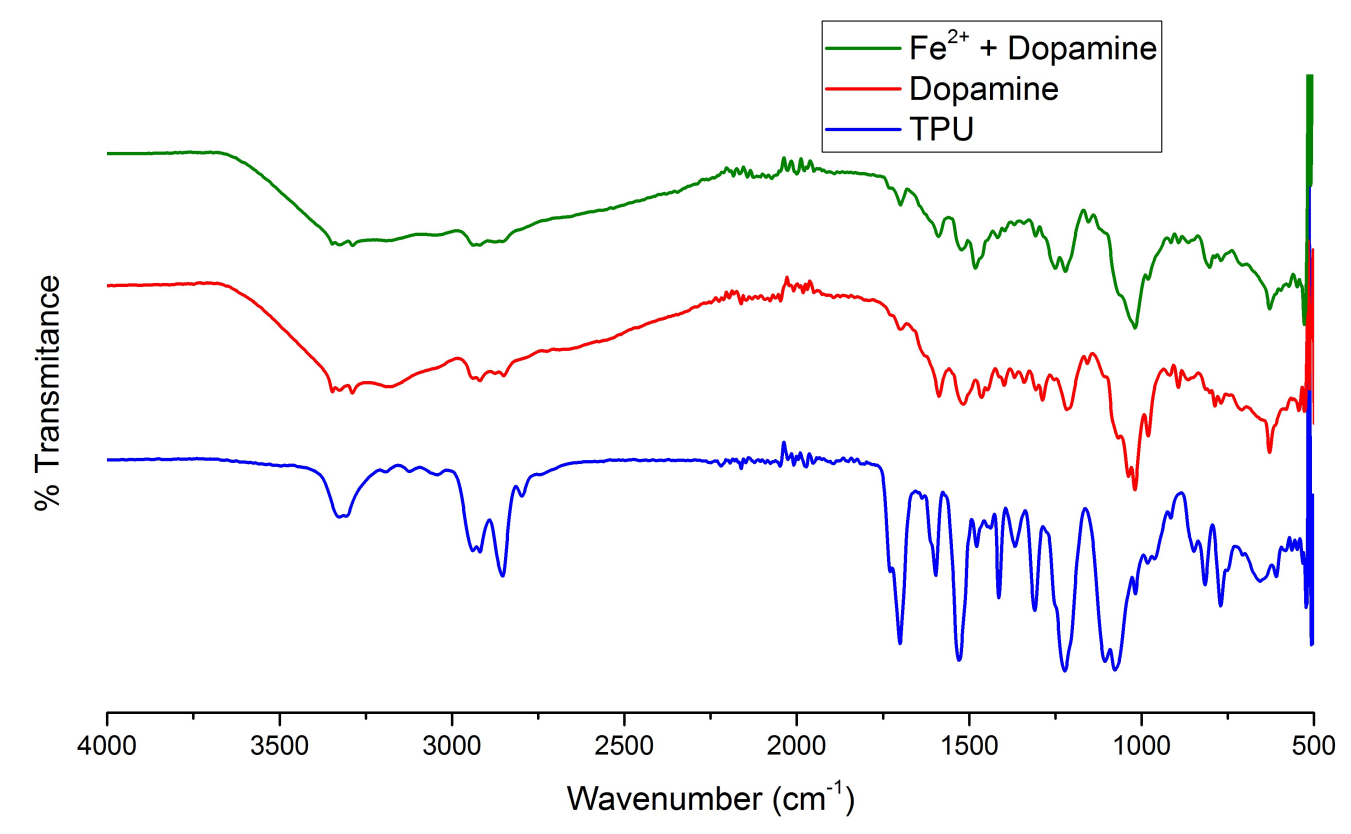

Figure 5.9: FTIR transmittance spectra for TPU (blue), dopamine coated TPU (red) and dopamine $\left(\mathrm{Fe}^{2+}\right)$ coated TPU (green). Assignments are found in Table 5.2. 
Table 5.2: FTIR assignments for bands found in Figure 5.9.

\begin{tabular}{|c|c|}
\hline Wavenumber $\left(\mathrm{cm}^{-1}\right)$ & Assignment \\
\hline \multicolumn{2}{|c|}{ TPU } \\
\hline 3325,1530 & $\nu(\mathrm{N}-\mathrm{H})$ \\
\hline $2942,2919,2850$ & $\nu(\mathrm{C}-\mathrm{H})$ \\
\hline 1730 sh, 1700 & $\nu(\mathrm{C}=\mathrm{O})$ \\
\hline Dopamine and dopamine $\left(\mathrm{Fe}^{2+}\right)$ \\
\hline 3300 br & $\nu(\mathrm{N}-\mathrm{H}), \nu(\mathrm{O}-\mathrm{H})$ \\
\hline $2942,2919,2850$ & $\nu(\mathrm{C}-\mathrm{H})$ \\
\hline $1700 \mathrm{w}$ & $\nu(\mathrm{C}=\mathrm{O})$ \\
\hline 1590,1520 & $\nu_{\text {ring }}(\mathrm{C}=\mathrm{C}), \nu_{\text {ring }}(\mathrm{C}=\mathrm{N})$, \\
\hline 1465 & $\nu_{\text {ring }}(\mathrm{C}=\mathrm{C})$ \\
\hline
\end{tabular}

coated TPU (12 hour coating). Important IR bands are listed in Table 5.2, with their mode assignments made with reference to infrared band tables and literature spectra [250, 251]. FTIR experimental details can be found in Section 3.2.6.

The spectrum for TPU is very similar to those found in the literature [250]. The features found at $3325 \mathrm{~cm}^{-1}$ and $1530 \mathrm{~cm}^{-1}$ are N-H stretching and bending modes respectively. Aliphatic $\nu(\mathrm{C}-\mathrm{H})$ stretching modes occur at 2942, 2919 and $2850 \mathrm{~cm}^{-1}$, and hydrogen bonded and free carbonyl stretching bands occur at $1730 \mathrm{~cm}^{-1}$ and $1700 \mathrm{~cm}^{-1}$ respectively. FTIR of polydopamine coated TPU shows a clear difference to that of the unmodified TPU. The broad peak between 3600 and $2500 \mathrm{~cm}^{-1}$ corresponds to both the N-H stretching mode and the $\mathrm{O}-\mathrm{H}$ stretching mode. The $\mathrm{C}-\mathrm{H}$ 
stretching modes observed are at the same wavenumbers as before coating. The introduction of more aromatic functionality means that more aromatic modes are observed in the range $1700-1300 \mathrm{~cm}^{-1}$ [251]. FTIR of dopamine $\left(\mathrm{Fe}^{2+}\right)$ coated TPU is almost identical to that of polydopamine coated TPU as expected from literature spectra [252].

XPS

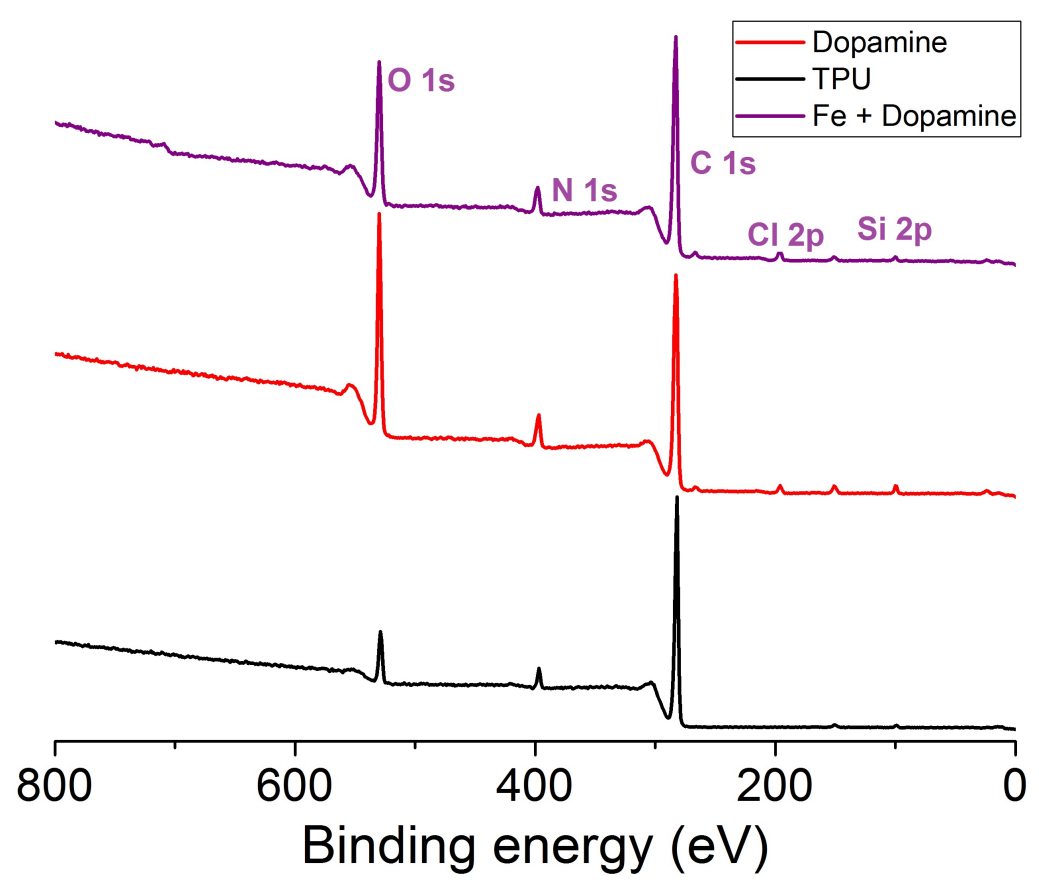

Figure 5.10: XPS surveys for TPU (black), dopamine coated TPU (red) and dopamine $\left(\mathrm{Fe}^{2+}\right)$ coated TPU (purple), showing $\mathrm{O} 1 \mathrm{~s}, \mathrm{~N} 1 \mathrm{~s}, \mathrm{C} 1 \mathrm{~s}, \mathrm{Cl} 2 \mathrm{p}$ and Si $2 p$ peaks. 
XPS provided chemical composition and chemical state information about the original membrane material and subsequent coatings. XPS is a surface sensitive technique that probes the first few nanometres of material in a sample. XPS signatures originating from $\mathrm{C}, \mathrm{N}, \mathrm{O}$ and $\mathrm{S}$ atoms were measured as well as wide scan spectra for each sample. The wide scan spectra (Figure 5.10) show three main signals; C 1s (285 eV), N 1s (397 eV) and O 1s (529 eV). Small additional signatures include Si $2 p(99.7 \mathrm{eV})$ and Cl 2p (199 eV).

Table 5.3: XPS survey assignments for uncoated TPU and dopamine coated TPU. Averaged over 5 spots, the error is the standard deviation.

\begin{tabular}{|c|c|c|c|c|c|c|c|}
\hline Pore coating & C 1s (\%) & N 1s (\%) & O 1s (\%) & Si 2p (\%) & Cl 2p (\%) & C:N & C:O \\
\hline TPU & $89.4 \pm 0.5$ & $3.1 \pm 0.9$ & $6.8 \pm 0.7$ & $0.7 \pm 0.2$ & - & 28.8 & 13.2 \\
\hline Dopamine & $67.8 \pm 1.9$ & $5.9 \pm 0.4$ & $22.9 \pm 1.5$ & $2.6 \pm 0.7$ & $0.7 \pm 0.3$ & 11.4 & 3.0 \\
\hline Dopamine(Fe $\left.{ }^{2+}\right)$ & $71.7 \pm 3.2$ & $5.9 \pm 0.3$ & $19.8 \pm 2.7$ & $1.2 \pm 0.1$ & $1.2 \pm 0.3$ & 12.1 & 3.6 \\
\hline
\end{tabular}

Uncoated TPU shows a $28.8 \mathrm{C}: \mathrm{N}$ ratio and a $13.2 \mathrm{C}: \mathrm{O}$ ratio, similar to other polyurethane samples examined in the literature [253]. The addition of dopamine and dopamine $\left(\mathrm{Fe}^{2+}\right)$ causes a decrease in both ratios as a polydopamine coating contains more nitrogen and oxygen atoms. Chemical composition including atomic percentages are shown in Table 5.3.

Figure 5.11 shows high resolution XPS peak fits for $\mathrm{O} 1$ s and $\mathrm{C} 1$ s peaks for the three different surfaces. Assignments of these peaks can be found 
Table 5.4: High resolution XPS assignments. Assignments were made in accordance with the polymer handbook [254] and XPS handbook [255].

\begin{tabular}{|c|c|}
\hline Functional group & Binding energy $(\mathrm{eV})$ \\
\hline \multicolumn{2}{|c|}{ TPU } \\
\hline \multicolumn{2}{|c|}{ C 1s } \\
\hline $\mathrm{C}-\mathrm{H}_{x}, \mathrm{C}-\mathrm{C}$ & 285.0 \\
\hline $\mathrm{C}-\mathrm{O}$ & 285.6 \\
\hline $\mathrm{HNCOO}$ & 287.9 \\
\hline \multicolumn{2}{|c|}{$\mathrm{N} 1 \mathrm{~s}$} \\
\hline $\mathrm{NCOO}$ & 397.2 \\
\hline \multicolumn{2}{|c|}{$\mathrm{O} 1 \mathrm{~s}$} \\
\hline $\mathrm{C}=\mathrm{O}$ & 528.8 \\
\hline NCOOC & 529.7 \\
\hline \multicolumn{2}{|c|}{ Dopamine } \\
\hline \multicolumn{2}{|c|}{$\mathrm{C} 1 \mathrm{~s}$} \\
\hline $\mathrm{C}-\mathrm{H}_{x}, \mathrm{C}-\mathrm{C}$ & 285.0 \\
\hline $\mathrm{C}-\mathrm{O} / \mathrm{C}-\mathrm{N}$ & 286.5 \\
\hline $\mathrm{HNCOO}$ & 288.2 \\
\hline$\pi-\pi^{*}$ satellite & 289.4 \\
\hline \multicolumn{2}{|c|}{ N 1s } \\
\hline $\mathrm{NCOO}$ & 397.2 \\
\hline $\mathrm{CNH}_{2}$ & 399.1 \\
\hline \multicolumn{2}{|c|}{$\mathrm{O} 1 \mathrm{~s}$} \\
\hline $\mathrm{C}=\mathrm{O}$ & 528.5 \\
\hline $\mathrm{NCOOC}$ & 529.8 \\
\hline $\mathrm{C}-\mathrm{O}-\mathrm{C}$ & 530.5 \\
\hline $\mathrm{C}-\mathrm{OH}$ & 531.2 \\
\hline \multicolumn{2}{|c|}{ dopamine $\left(\mathrm{Fe}^{2+}\right)$} \\
\hline \multicolumn{2}{|c|}{$\mathrm{C} 1 \mathrm{~s}$} \\
\hline $\mathrm{sp}^{2}$ & 283.88 \\
\hline $\mathrm{C}-\mathrm{H}_{x}, \mathrm{C}-\mathrm{C}$ & 285.0 \\
\hline $\mathrm{C}-\mathrm{O} / \mathrm{C}-\mathrm{N}$ & 286.3 \\
\hline $\mathrm{HNCOO}$ & 287.9 \\
\hline$\pi-\pi^{*}$ satellite & 288.7 \\
\hline \multicolumn{2}{|c|}{$\mathrm{N}$ 1s } \\
\hline $\mathrm{NCOO}$ & 397.6 \\
\hline $\mathrm{CNH}_{2}$ & 399.2 \\
\hline \multicolumn{2}{|c|}{ O 1s } \\
\hline $\mathrm{C}=\mathrm{O}$ & 528.5 \\
\hline $\mathrm{NCOOC}$ & 530.1 \\
\hline $\mathrm{C}-\mathrm{O}-\mathrm{C}$ & 530.9 \\
\hline
\end{tabular}



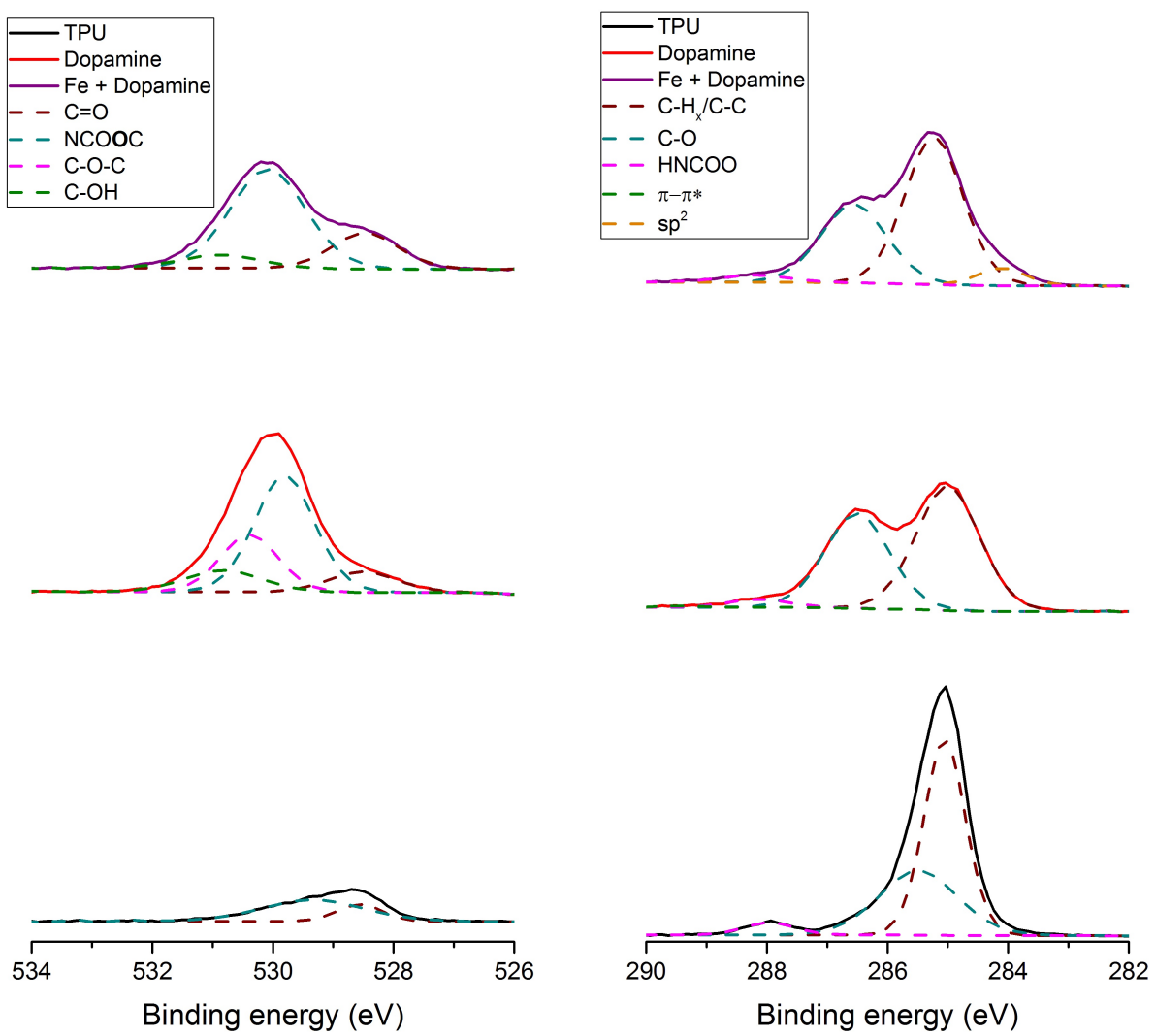

Figure 5.11: High resolution XPS peaks for $\mathrm{O}$ 1s (left) and $\mathrm{C}$ 1s (right). From bottom to top for TPU (black), dopamine coated TPU (red) and dopamine $\left(\mathrm{Fe}^{2+}\right)$ coated TPU (purple). Peak assignments can be found in Table 5.4 

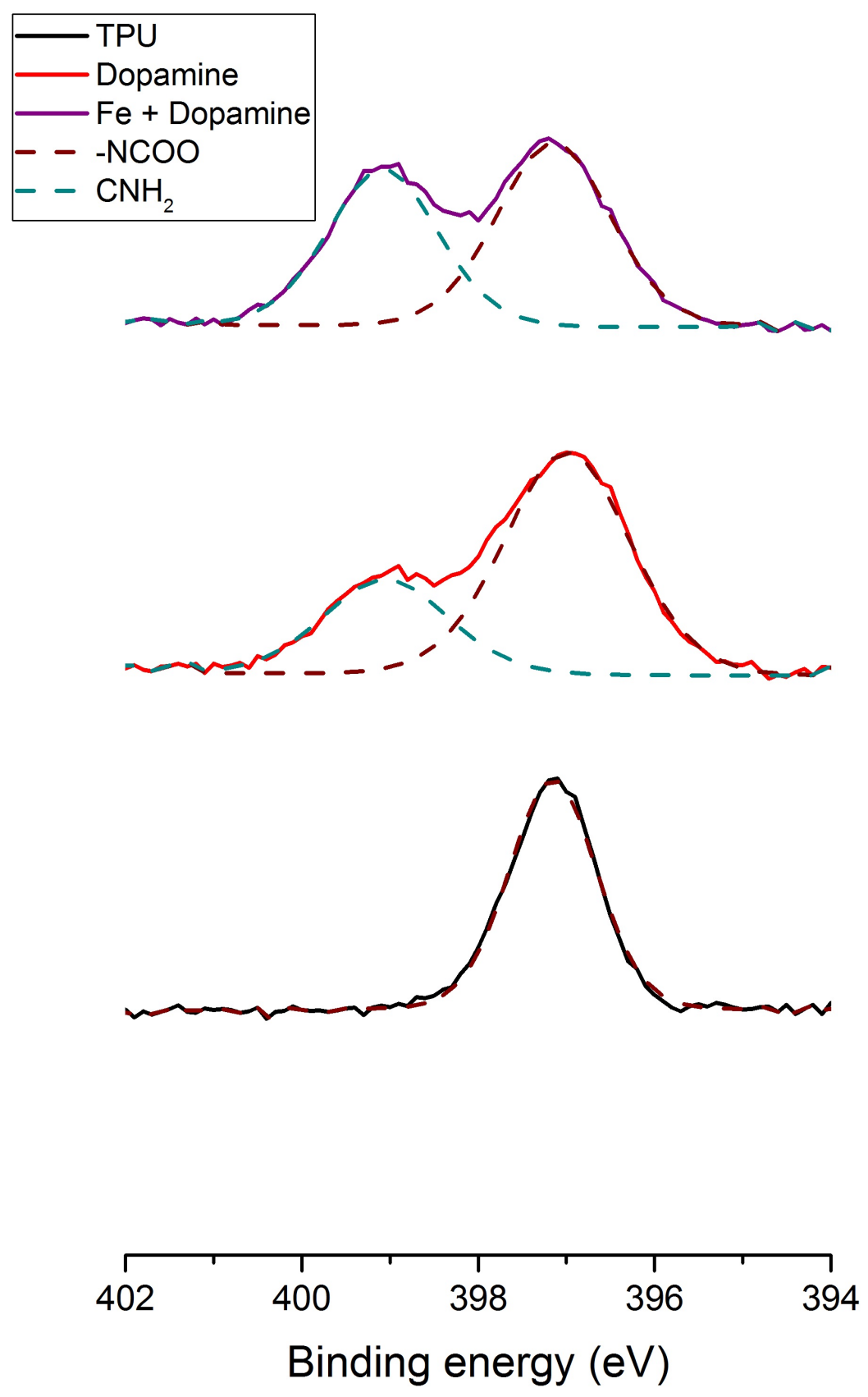

Figure 5.12: High resolution XPS peak first for N 1s. From bottom to top for TPU (black), dopamine coated TPU (red) and dopamine $\left(\mathrm{Fe}^{2+}\right)$ coated TPU (purple). Peak assignments can be found in Table 5.4 
in Table 5.4. It can be seen that the introduction of dopamine increases the amount of oxygen atoms present in the sample and the different environments oxygen is found in. The dopamine and dopamine $\left(\mathrm{Fe}^{2+}\right)$ samples show C-O-C and C-OH components whereas the TPU sample only contains $\mathrm{C}=\mathrm{O}$ and NCOOC components. The carbon signature also clearly changes on the addition of dopamine. A comparatively larger C-O/C-N peak and the addition of $\mathrm{sp}^{2}$ and aromatic carbons is seen. $\mathrm{N} 1$ s signatures show a move from a sole $-\mathrm{NCOO}$ peak to the introduction of a $-\mathrm{CNH}_{2}$ peak (Figure 5.12).

These XPS results continue to confirm the successful coating of the pore with dopamine, including the addition of $\mathrm{Fe}^{2+}$.

\section{$I-V$ Curves and $\zeta_{\text {pore }}$ Measurements}

The current-voltage relationship is a straightforward way to monitor changes in pore surface charge. ICR is the name given to the phenomenon where an asymmetric current-voltage curve is observed, due to the current at one voltage polarity being larger than the current at the same absolute voltage of opposite bias [256]. ICR has been seen in a variety of biological and 
synthetic pores. Biological pores such as those present in lipid membranes have been shown to rectify current, as have artificial pores inserted into lipid membranes [257]. Siwy et al. were the first to show current rectification for purely synthetic pores [257-259]. In a number of studies in the early 21st century, track etched conical polyethylene terephthalate pores were shown to give diode-like asymmetric currents at $\mathrm{pH}$ values above the isoelectric point of the material ( $\mathrm{pH}$ 3.8) and in dilute electrolytes (less than $3 \mathrm{M} \mathrm{KCl}$ ) [259]. The pores were fabricated to mimic biological pores found in lipid membranes [257]. The conditions required to achieve ICR are listed as the opening diameter of the tip being a comparable size to the thickness of the EDL, the presence of excess surface charge on the pore walls, and asymmetry of the ion distribution within the pore [256]. ICR is observed in TRPS despite the pore diameter being much larger than the EDL thickness.

Depending on the sign of the excess surface charge a different ICR signature will be observed (Figure 5.13). A number of explanations for ICR have been presented. The ion depletion and accumulation model (similar to that described in Chapter 6) studied by Lan et al. [260] suggests that a negatively charged pore has a cation selective small pore opening and 


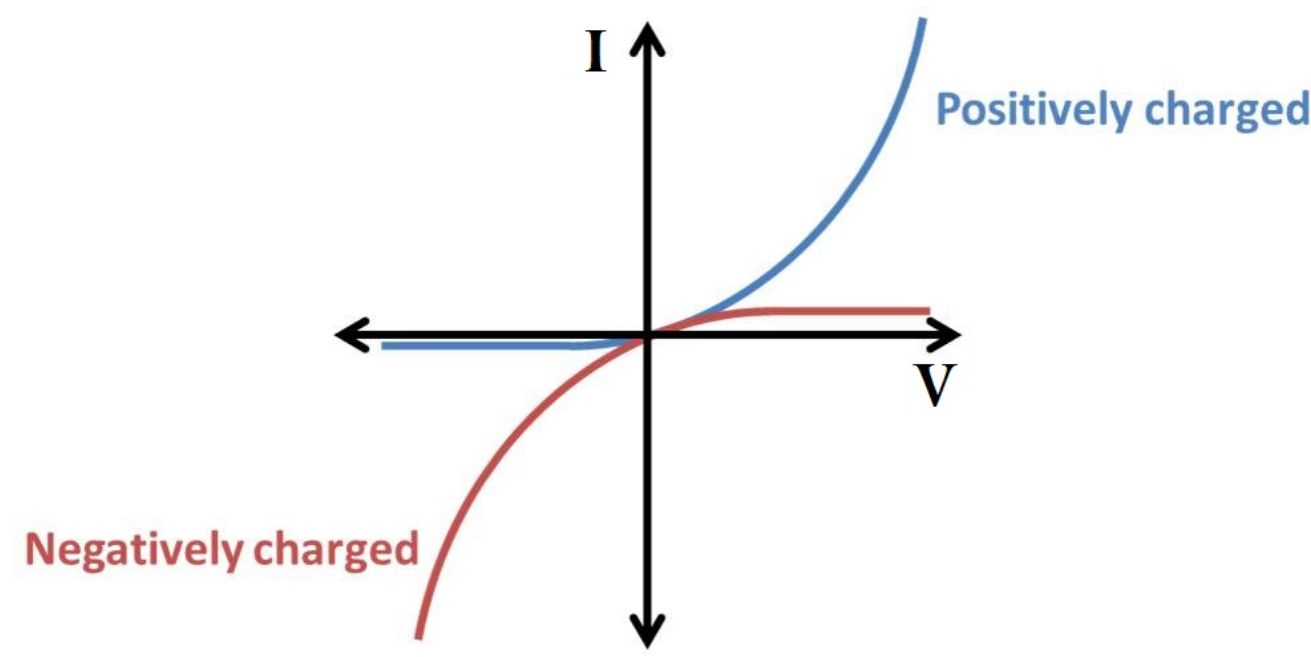

Figure 5.13: Schematic of ICR $I-V$ curves for highly positive and highly negatively charged pores.

treats the large pore opening as part of the bulk. At negative voltages positive ions are directed from the bulk into the pore and negative ions move in the opposite direction. Due to the cation selective nature of the small pore opening there is a build up of both cations and anions inside the pore and an increase in conductivity relative to the bulk. At positive voltages negative ions are directed from the bulk into the pore and positive ions move in the opposite direction. However, negative ions are rejected at the small pore opening and there is a build up of charge outside the pore which leads to a decrease in nanopore conductivity and a very low current at positive voltages. A positively charged pore will be anion selective and therefore have the opposite result. 
(a)
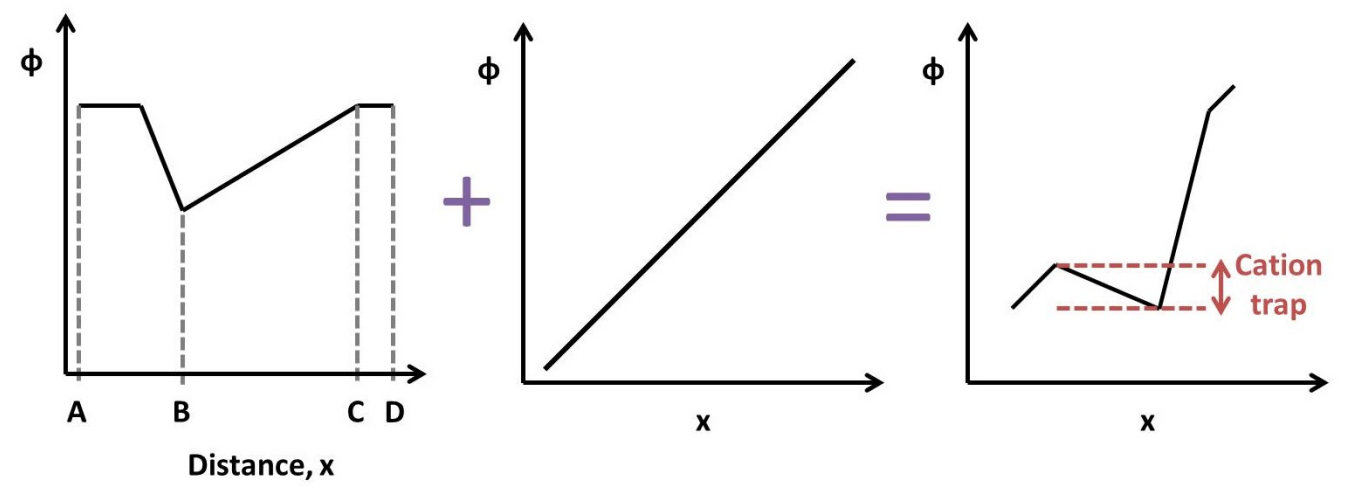

(b)
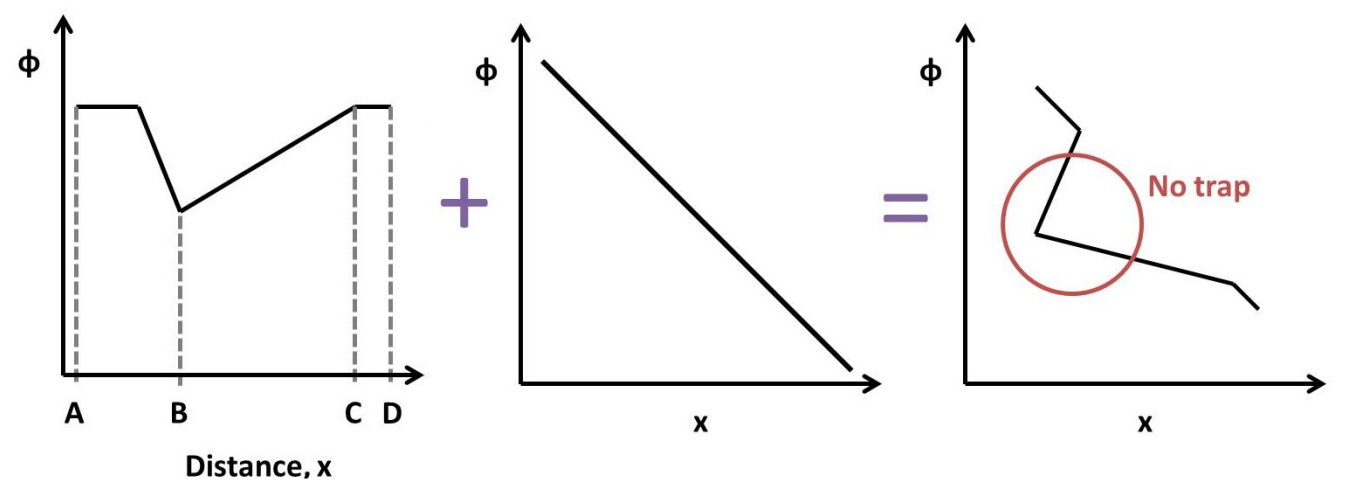

Figure 5.14: Schematic of the ratchet model(a) with cation trap and (b) without cation trap. The far left plot represents the potential due to surface charge, the middle plot represents the potential due to the applied potential and the far right plot represents the combination of the first two. The vertical axis scales are equal across the plots. Region A-B represents the region just outside the tip of the pore, region $B-C$ represents the small part of the pore where the radius is comparable to the double-layer thickness, region C-D represents the part of the nanopores where the radius is much greater than the double-layer thickness and the pore charge no longer has an effect on the potential. 
Another explanation for ICR is the ratchet model. Conical nanotubes with a negative surface charge give a single ratchet energetic 'tooth' for a traversing cation (Figures 5.14 ). When a positive potential is applied across the membrane the voltage drop caused by the resulting positive current is superimposed on the potential across the pore due to the applied voltage. This results in an electrostatic trap for the cation at point B which causes the low current "off" state at positive potentials (Figure 5.14a). When a negative potential is applied across the membrane the voltage drop caused by the resulting negative current results in no electrostatic trap for the cation at and leaves the pore in an "on" state (Figure 5.14b).

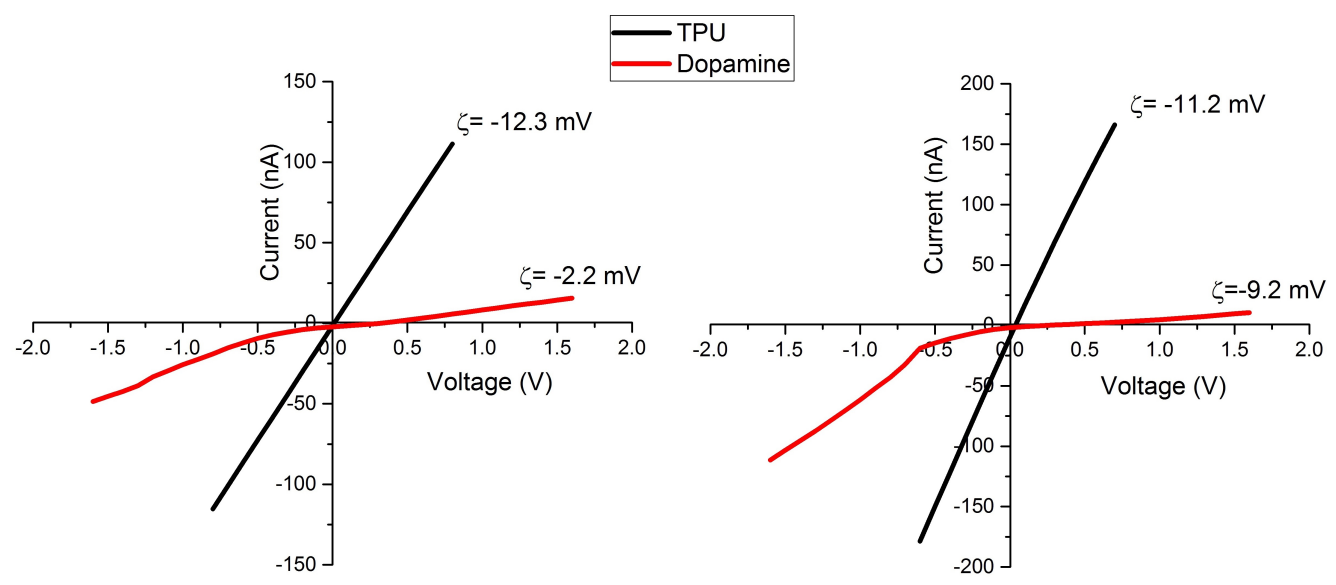

Figure 5.15: ICR before and after dopamine coating two separate NP200 pores in $100 \%$ PBS at $46 \mathrm{~mm}$ stretch. $\zeta_{\text {pore }}$ values from streaming potential measurements are shown on the plot. Data points were taken every $0.1 \mathrm{~V}$.

Under usual TRPS working conditions the ohmic relationship between 
voltage and current holds and no ICR is observed. On the addition of dopamine ICR is observed (Figure 5.15). It is expected that the deprotonated catechol hydroxyl groups of polydopamine would result in a negative surface charge. The ICR observed indicates the pore is more negatively charged than the uncoated pore following the pattern seen in Figure 5.13. Streaming potential measurements however measure a less negative $\zeta_{\text {pore }}$. This is thought to be due to uneven coating of the pore surface giving misleading $\zeta_{\text {pore }}$ results. This uneven coating might also explain why the $I-V$ trace is not smooth and contains some kinks.

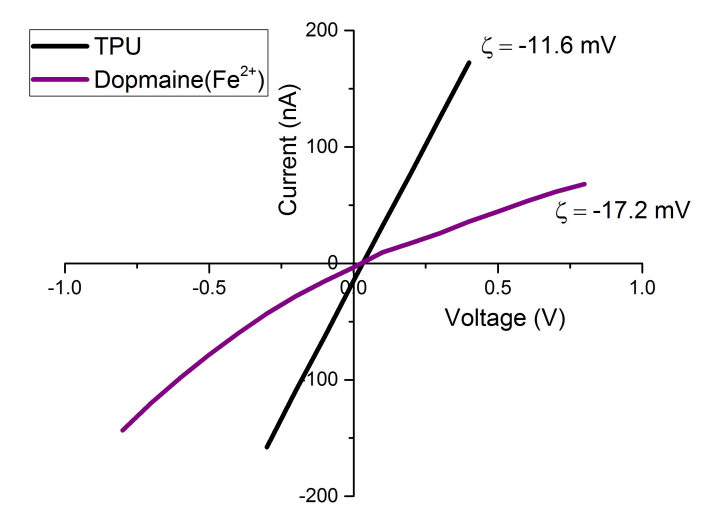

Figure 5.16: ICR before and after dopamine $\left(\mathrm{Fe}^{2+}\right)$ coating an NP200 pore at a $45 \mathrm{~mm}$ stretch. Data points were taken every $0.1 \mathrm{~V}$.

The same experiment was carried out with dopamine $\left(\mathrm{Fe}^{2+}\right)$ coated pores (Figure 5.16). The ICR observed here resembles that of a negatively charged pore. This is also seen in the streaming potential measurements. It is 
thought that the addition of a metal ion, which can co-ordinate to dopamine, resulted in a more even coating hence the agreement between streaming potential and ICR measurements.

\subsubsection{Polymer Brush Functionalisation}

Pores were functionalised with polyhydroxyethylmethacrylate (PHEMA) and PAA using the Graftfast method [261, 262]. Graftfast involves chemical reduction of diazonium salts by reducing agents in the presence of the vinylic monomer. It is a one-pot process that can be performed in aqueous solution at ambient pressure and temperature. PHEMA and PAA coated pores were received from the lab of Prof. Jadranka Travas-Sejdic at the University of Auckland. PHEMA coated pores were expected to be more neutral and PAA coated pores more negatively charged.

In Figure 5.17 the streaming potential measurements taken on these pores are presented. It can be seen that the PAA pores of the same size as the PHEMA pores are more negatively charged as expected. There is a drop in charge with pore size (similar to that seen in Figure 5.3) for the PAA coated pores, possibly due to these pores not wetting during 


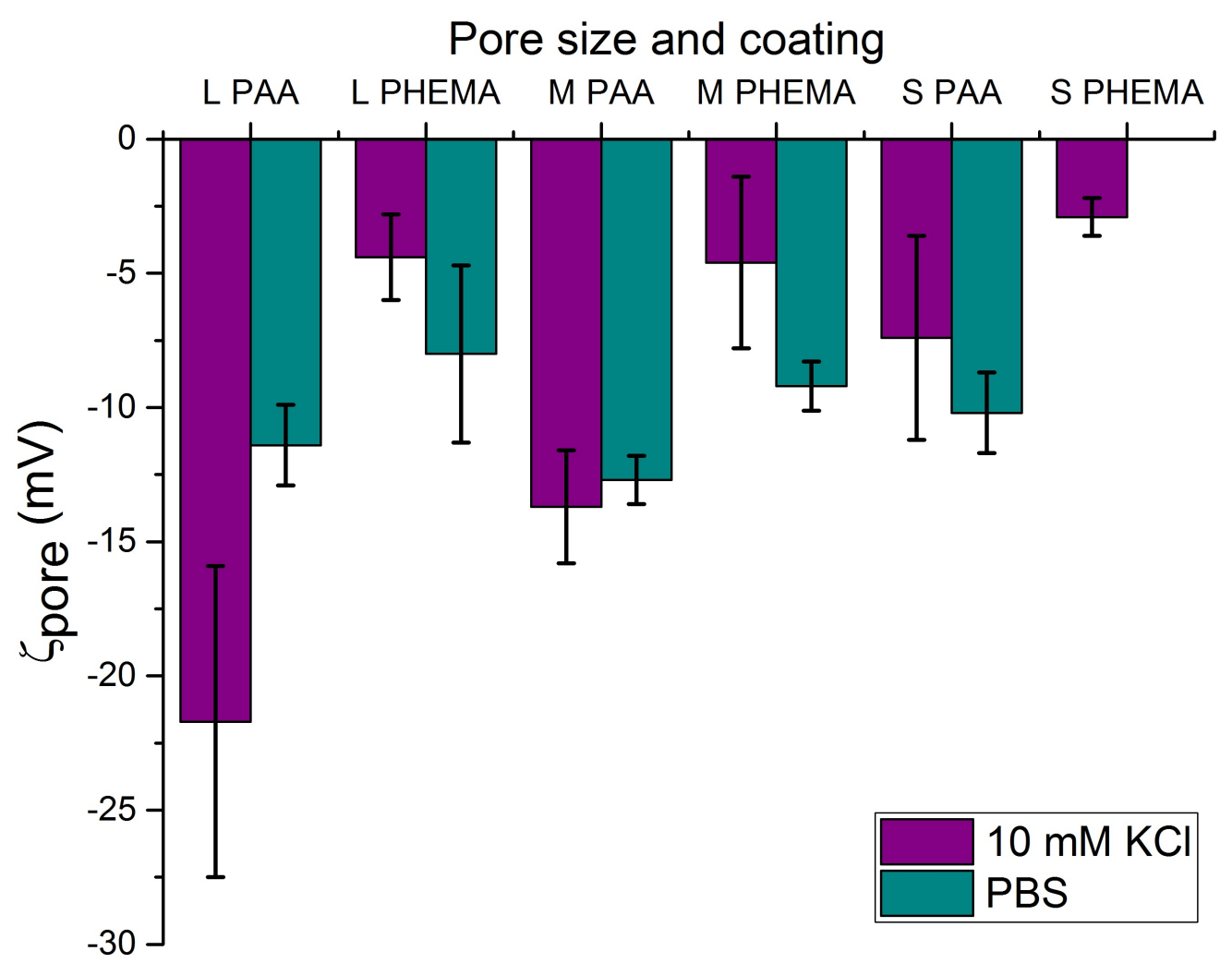

Figure 5.17: Bar chart showing $\zeta_{\text {pore }}$ for different pore sizes and pore coatings using two different electrolytes. L refers to large pores (NP2000), M refers to medium pores (NP400) and S refers to small pores (NP100). Error bars indicate the standard deviation of 5 measurements. 
the streaming potential measurement process. The streaming potential of smaller pores was more difficult to measure. There was more drift in voltage observed with smaller pores. Other experimental artefacts such as the measured pore charge changing bias were also experienced. These could sometimes be counteracted by increasing pore "soaking time" prior to measurement. The small PHEMA pore couldn't be measured in PBS because of these experimental difficulties. $\zeta_{\text {pore }}$ is expected to be greater for PBS than for $10 \mathrm{mM} \mathrm{KCl}$ due to the ionic strength but this wasn't always the case. Other factors such as $\mathrm{pH}$, temperature, substrate material, and counterion type also affect the relationship of zeta potential with electrolyte and may have been the reason for the difference.

\subsubsection{PEI/PAA}

Layer-by-layer coating assembly involves the sequential absorption of polymers onto a surface from solution giving a multi-layered coating. Usually the layers are polyelectrolytes assembled via electrostatic interactions between the charged surface and polyelectrolyte, and then between differently charged polyelectrolytes. The method is simple and versatile and has therefore been used to coat a number of substrates including noble 


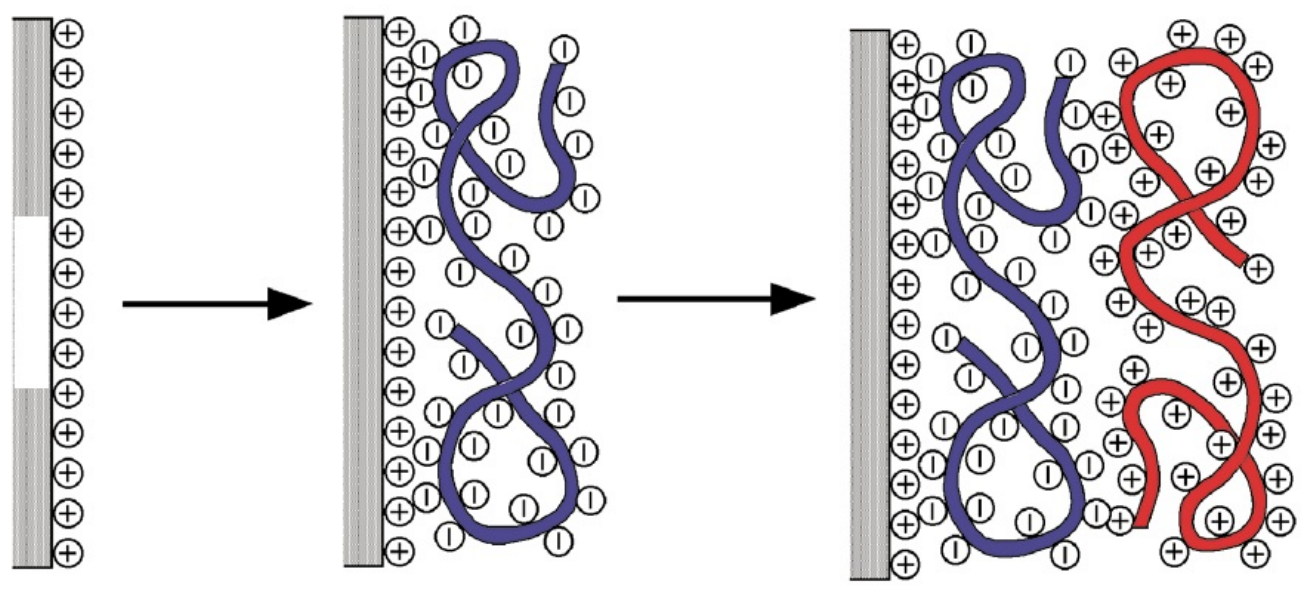

Figure 5.18: Schematic showing layer-by-layer coating of a surface using polyelectrolytes and electrostatic interactions. Adapted from [263].

metals, metal oxides and synthetic polymers [264, 265].

Here, a slight modification of the layer-by-layer coatings used by Blundell et al. [113] is used to coat TPU pores. The negatively charged TPU pore is first coated with the cationic polymer PEI followed by the anionic polymer PAA. $I-V$ curves along with $\zeta_{\text {pore }}$ measurements were used to confirm the different coatings as 2 alternating PEI/PAA layers were added. The surface charge was expected to become more positive when PEI was added and more negative when PAA was added.

Figure 5.19 and Table 5.5 summarize rectification measurements at 3 different electrolyte concentrations, $10 \%, 50 \%$ and 100\% PBS, at different 

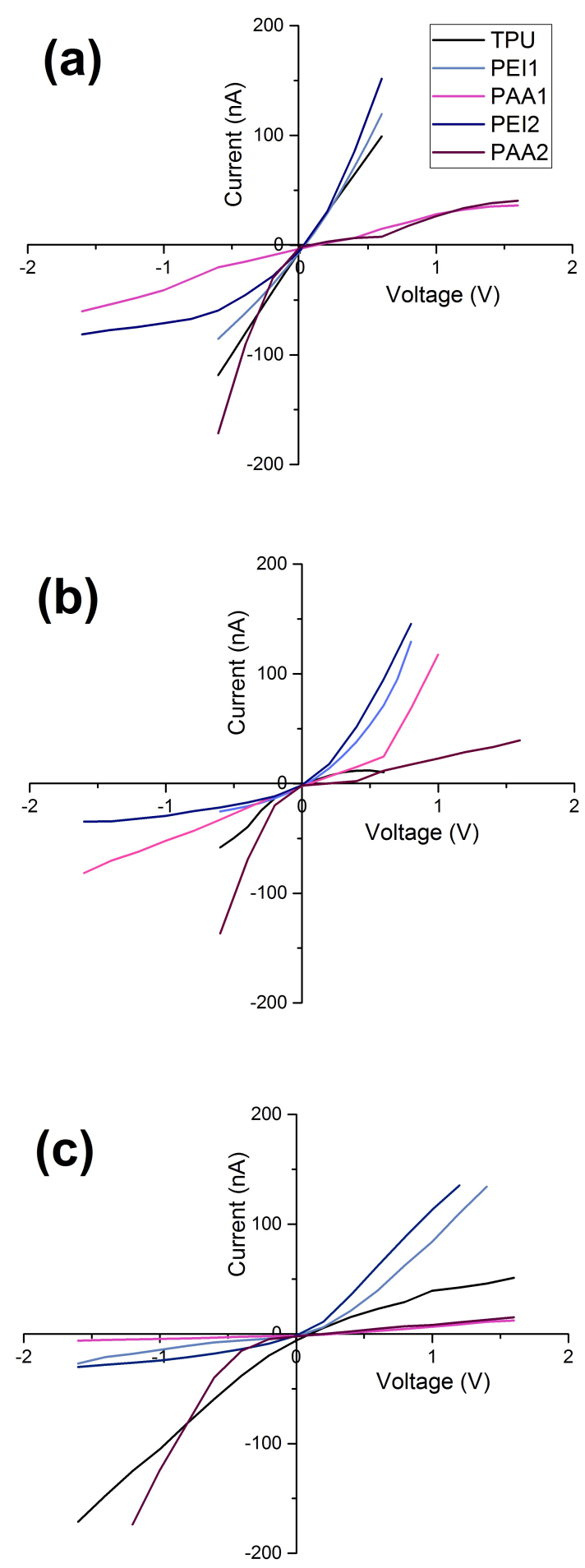

Figure 5.19: ICR switch between positive and negative charged surface using PEI/PAA layer-by-layer coating at (a) 100\% PBS, (b) 50\% PBS and (c) $10 \%$ PBS. Rectification ratios and $\zeta_{\text {pore }}$ values are listed in Table 5.5. Measurements were taken on a single NP150 pore at $45 \mathrm{~mm}$ stretch. Data points were taken every $0.1 \mathrm{~V}$ 
stages throughout the coating process. ICR patterns can be quantified as a rectification ratio $\left(\mathrm{R}_{f}\right)$ which is defined here as the current at positive voltage as a fraction of the current at equivalent negative voltage. $\mathrm{R}_{f}$ is less than 1 when surface charge is negative, $R_{f}$ is equal to 1 when surface charge is neutral and $R_{f}$ is more than 1 when surface charge is positive. Streaming potential measurements were also taken at each stage of the coating process.

Table 5.5: $\zeta_{\text {pore }}$ values and rectification ratios $\left(R_{f}\right)$ for PEI/PAA coated pores at 3 different electrolyte concentrations. 1 indicates the first layer applications. 2 indicates the second layer applications.

\begin{tabular}{|c|c|c|c|}
\hline Coating & Electrolyte (\%PBS) & $\zeta_{\text {pore }}(\mathrm{mV})$ & $\mathrm{R}_{f}$ at $0.6 \mathrm{~V}$ \\
\hline None & $10 \%$ & -13.2 & 0.38 \\
\hline None & $50 \%$ & -9.6 & 0.18 \\
\hline None & $100 \%$ & -9.0 & 0.84 \\
\hline PEI 1 & $10 \%$ & +6.1 & 5.11 \\
\hline PEI 1 & $50 \%$ & +5.6 & 5.07 \\
\hline PEI 1 & $100 \%$ & +6.3 & 1.39 \\
\hline PAA 1 & $10 \%$ & +1.5 & 0.76 \\
\hline PAA 1 & $50 \%$ & +2.7 & 0.74 \\
\hline PAA 1 & $100 \%$ & -17.5 & 0.73 \\
\hline PEI 2 & $10 \%$ & +4.5 & 3.4 \\
\hline PEI 2 & $50 \%$ & +8.9 & 4.4 \\
\hline PEI 2 & $100 \%$ & +21.9 & 2.5 \\
\hline PAA 2 & $10 \%$ & +0.8 & 0.11 \\
\hline PAA 2 & $50 \%$ & -1.6 & 0.08 \\
\hline PAA 2 & $100 \%$ & -3.5 & 0.04 \\
\hline
\end{tabular}

It can be seen that the ICR patterns switch between the positive and negative signatures when PEI and PAA are added respectively. The sig- 
natures are clearer at lower electrolyte concentrations when higher levels of rectification are expected. In some cases the addition of PAA produces inconsistency between the values of $\zeta_{\text {pore }}$ (positive) and $R_{f}(<1)$ showing that this coating doesn't always cover the entire pore. The $\mathrm{R}_{f}$ values are as expected for each coating layer indicating the expected surface charge bias. The magnitude of the $\zeta_{\text {pore }}$ value measured did not always align with magnitude of the $\mathrm{R}_{f}$ value measured but they always indicated the same directional change in charge when a new layer was added.

\subsection{Oscillations}

Oscillating ionic currents have been observed previously with conical pores and were attributed to nanoprecipitation. The transient formation and re-dissolution or migration of precipitates due to intra-pore ion concentrations and applied voltages was thought to temporarily block the pore causing the large (larger than a translocating particle) temporary decreases in current [266]. As ion concentrations within the charged pore are different to those in the bulk solution, different solubility properties are seen within the pore. Because applied voltage also influences the number of 
ions in the pore, solubility can also be seen to be voltage dependant. In this Section, the first report of possible nanoprecipitation seen in TRPS is presented.

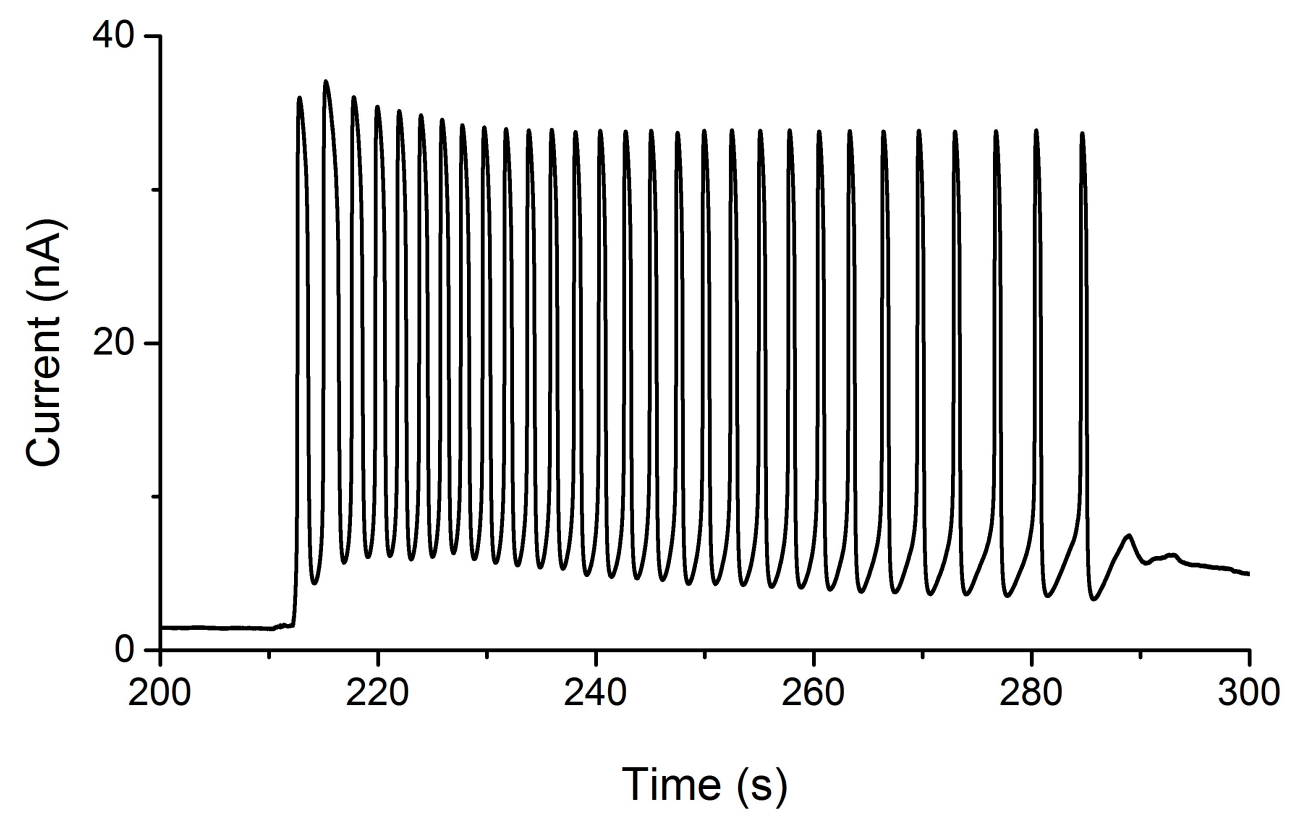

Figure 5.20: Example of large current oscillations in TRPS following a 0 to $1.6 \mathrm{~V}$ switch.

In TRPS experiments oscillations were sometimes observed with layerby-layer coating after both PEI and PAA layers had been added. Oscillations were often observed when voltage or stretch were varied suddenly (Figures 5.20 and 5.21). One explanation is that the precipitation 
of PEI/PAA complexes is the cause of the observed oscillations. Under a negative voltage the small pore opening is depleted of ions and thus the individual polyelectrolytes will have less counterions balancing their charge. This may lead to the oppositely charged polyelectrolytes coming together to form complexes that temporarily block the pore before either re-dissolving or migrating out of the pore. Polyelectrolyte complexes have elaborate phase behaviour that depends on a range of factors including mixing ratio, ionic strength, $\mathrm{pH}$ and mixing conditions. Oscillations were somewhat sporadic and difficult to isolate. Dispite these issues oscillation frequency was seen to increase with applied potential (Figure 5.21) which has been reported previously for nanoprecipitation [266].

An alternative explanation is that these oscillations are due to a change in charge state of the pore with applied voltage or stretch. It may be that a pore coating is spread differently depending on the stretch of the pore and that at a certain stretch the ion selectivity of that pore changes and a transition state can be captured where oscillations occur.

More investigation is needed here as oscillations are difficult to capture, and vary considerably in their magnitude, frequency and longevity from instance to instance. 

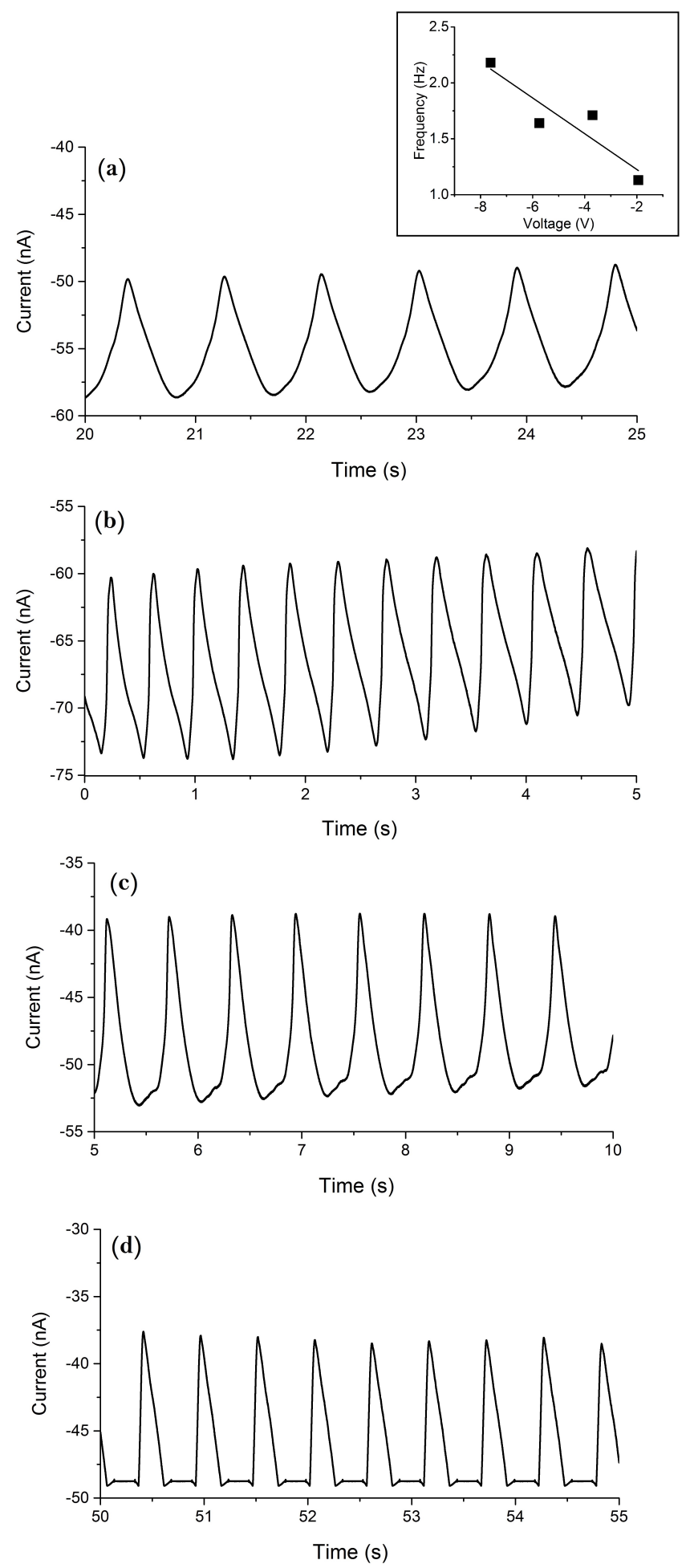

Figure 5.21: Large oscillations seen with sudden voltage decreases from 0 $\mathrm{V}$ to (a) $-1.94 \mathrm{~V}$ (b) $-3.70 \mathrm{~V}$ (c) $-5.74 \mathrm{~V}$ and (d) $-7.61 \mathrm{~V}$ in PEI/PAA coated pores in $10 \%$ PBS. Measurements taken on a single NP150 pore at $45 \mathrm{~mm}$ stretch. Inset shows oscillation frequency against voltage. 


\subsection{Conclusion}

In this Chapter $\zeta_{\text {particle }}$ measurements using Method IV were continued with the addition of streaming potential measurements so that $\zeta_{\text {pore }}$ could be obtained using the qNano. $\zeta_{\text {pore }}$ measurements were made at a range of different pore sizes and then combined with $\zeta_{\text {particle }}$ measurements to show that charge measurements could be made at a range of stretches. $\zeta_{\text {pore }}$ and $\zeta_{\text {particle }}$ measurements were made at a range of different electrolyte concentrations and pHs. The isoelectric point of TPU was obtained. Random uncertainties using Method IV have been shown to be less than previously investigated methods and the inclusion of measured calibration $\zeta_{\text {particle }}$ and $\zeta_{\text {pore }}$ values reduces the systematic uncertainty.

In this Chapter, although uncertainties were better characterized and understood than Chapter 4, trends with respect to $\mathrm{pH}$, electrolyte concentration and manufacturers specifications for charge were unclear, as they were for DLS measurements. These represent some of the ongoing issues for $\zeta$-potential measurements along with measurement and control of pore surface charge.

This Chapter and the previous Chapter represent a thorough investi- 
gation into charge measurements using TRPS. For the most part, previous investigations of $\zeta_{\text {particle }}$ measurement using TRPS have only compared a few particle types in one set of conditions $[14,112,114]$. These previous studies also use a single pre-assigned value of $\zeta_{\text {pore }}$ even when working in biological conditions which can affect pore surface properties. The work presented in these two Chapters builds on other detailed investigations of charge measurement with TRPS [47, 111], by examining a large number of particle sets in a range conditions, as well as addressing the issue of measuring $\zeta_{\text {pore }}$.

In addition to particle charge measurements, a number of types of coatings were successfully applied to TPU pores. Pore coatings were analysed using XPS, FTIR, $I-V$ curves and streaming potential measurements. Pore coatings have not been previously investigated using TRPS so are an interesting expansion of the current technology. However, in this research coating removed the ability of a pore to measure particles. Streaming potential measurements of coated pores in general agreed with ICR in terms of whether the pore was negatively or positively charged. Often ICR was a more consistent indicator of surface charge especially for dopamine coated pores where the ICR indicated a more negatively charged pore but $\zeta_{\text {pore }}$ 
indicated a more positive one. This is most likely because of uneven pore coating of the complicated pore geometry. Large current oscillations were observed with coated pores, possibly due to nanoprecipitation. Improvement of pore coatings to allow for particle measurement and complete coating of the pore (so that ICR agrees with $\zeta_{\text {pore }}$ ), as well as further investigation of the large current oscillations are all areas for future research. 


\section{Chapter 6}

\section{Biphasic Pulses}

\subsection{Introduction}

In this Chapter the first study of biphasic pulses using TRPS is presented. A TRPS pulse is conventionally resistive (Figure 1.1), but conductive pulses are observed under certain experimental conditions, as well as biphasic pulses which include both resistive and conductive components. In this work the effect of a number of experimental variables on the presence and form of biphasic pulses was investigated. The experimental variables investigated here include the concentration of the PBS electrolyte, particle 
charge, pore size, applied voltage, and the direction of particle motion. Besides providing fundamental understanding, the results are important for the TRPS technique because variations in pulse geometry can affect conventional TRPS particle characterization as this is briefly investigated (e.g. size, concentration and charge measurements). Biphasic pulses can also provide new information about particles not previously available with purely resistive pulses.

Much of the material in this Chapter has been published in [236]. All of the experiments and analysis were performed by Weatherall. Willmott developed the electrolyte concentration model and provided discussion and suggestions on writing style and the content of this paper.

\subsection{Background}

Conductive pulses in which the resistance transiently decreases, or biphasic pulses consisting of both a conductive and resistive component, have been reported for sensing of DNA using silicon-based pores [267-272]. These conductive pulses are most simply attributed to the introduction of counter-ions shielding the strong charge on the DNA backbone, pro- 
viding a positive contribution to the local conductivity and therefore ionic current [269]. RPS research at molecular scales can employ explicit molecular dynamics models to predict the structure of resistive pulses in detail. For example, the electrophoretic motion of DNA can be modelled [273, 274].

Recently, conductive and biphasic pulses have also been reported for larger particles. Gold nanoparticles 10-20 nm in diameter have produced biphasic pulses using silicon nitride pores [275], and nanopipettes [276]. Conductive pulses generated by a $300 \mathrm{~nm}$ hydrogel [194] and a $\sim 1 \mu \mathrm{m} \mathrm{mi-}$ crogel were attributed to high conductance of the particles relative to the electrolyte. The groups that carried out these gel studies each followed up with a study of biphasic pulses in which, somewhat remarkably, the particles used were standard polystyrene particles (mostly carboxylatefunctionalized) in the size range 120 - $470 \mathrm{~nm}[87,277]$. Unlike previous reports of conductive and biphasic pulses, neither the size of the EDL of the pore relative to the pore diameter nor the particle bulk conductivity should be significant in these experiments. Lan et al. [87] used conical glass pores of opening diameter $430 \mathrm{~nm}$ and length 50-100 $\mu \mathrm{m}$, while Menestrina et al. [277] used cylindrical polyethylene terephthalate pores of 
opening diameter $500-1500 \mathrm{~nm}$ and length $12 \mu \mathrm{m}$. These two studies provide the main comparisons for experiments outlined in this Chapter, along with their accompanying FEM results.

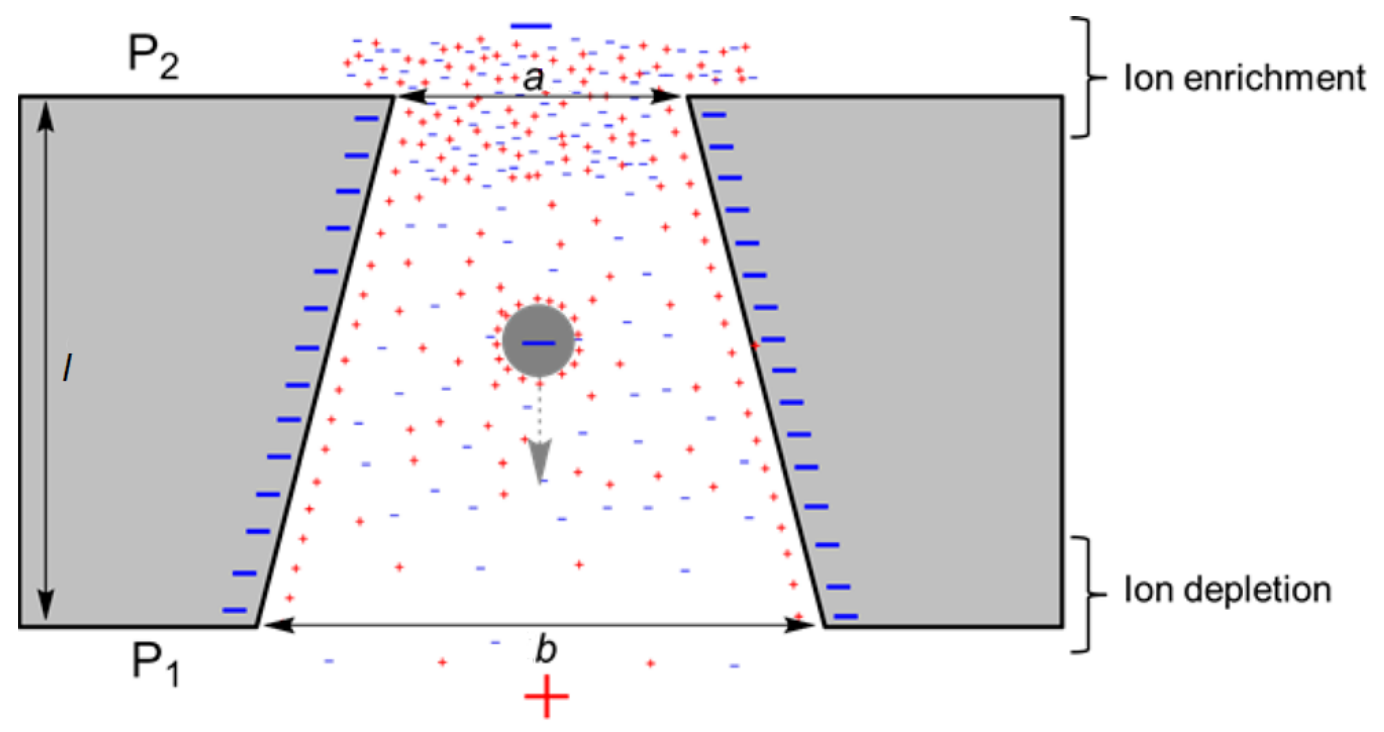

Figure 6.1: Schematic cross section through a membrane of thickness $l$, showing a conical pore (opening diameters $a$ and $b$ ) containing a particle with potential $V_{0}$ and pressure $P_{2}-P_{1}$ applied across the membrane. Both the particle and the pore have negative surface charge. Ionic concentration polarization is qualitatively indicated by the distribution of "+" and "-" signs.

The mechanism for conductive and biphasic pulses at submicron length scales [87, 277] is thought to involve competition between volume exclusion by the particles, which is the conventional mechanism for generating resistive pulses, and the effect of the particle surface charge on the ionic distribution near the pore, which can temporarily increase the pore 
conductivity. The ionic distribution in the pore is affected by concentration polarization, the phenomenon responsible for ICR in asymmetric nanopores [256]. When a potential is applied across a negatively charged pore, positive ions move freely from one fluid cell to the other, while negative ions are electrostatically repelled by the membrane. This leads to an increase in ionic concentration near the cathode and a decrease in ionic concentration near the anode (Figure 6.1).

\subsection{Results and Discussion}

The four particle sets studied here are sets A-D (Table 3.3) of carboxylated polystyrene beads purchased from Bangs Laboratories (Fishers, IN, USA). Particle set D was used for all experiments other than in Sections 6.3.2 and 6.3.6 (where all four particle sets were used), as this particle set gave the largest conductive pulses which were therefore the easiest to monitor. Particle sets A-D have diameters close to $200 \mathrm{~nm}$, but the nominal surface group density varies. The manufacturer-supplied surface group density is defined in terms of the moles of $-\mathrm{COOH}$ functional group bound to the surface per gram of particles $\left(\mu \mathrm{eq}^{-1}\right)$, calculated from titrations during 
manufacturing as described in [278].

Conditions for the experiments in this Chapter are summarized in Table 6.1, including the parameter varied in each case.

Table 6.1: Summary of conditions for each experiment in this Chapter.

\begin{tabular}{|c|c|c|c|c|c|}
\hline Section & Parameter varied & $V_{0}(\mathrm{~V})$ & $P(\mathrm{~Pa})$ & $X(\mathrm{~mm})$ & $a(\mathrm{~nm})$ at $X=45$ \\
\hline 6.3 .1 & Configuration & $-1.6 /+1.6$ & 590 to 980 & 49.5 & 600 \\
\hline 6.3 .1 & Pressure & -1.6 & -1300 to 0 & 52.0 & 158 \\
\hline 6.3 .2 & Charge & -1.6 & 0 & 47.0 & 152 \\
\hline 6.3 .3 & Electrolyte & -0.4 & 0 & 49.0 & 176 \\
\hline 6.3 .4 & Potential & -1.6 to 1.6 & 0 & 49.0 & 176 \\
\hline 6.3 .5 & Pore size & -0.7 & 0 & 46.5 to 50.0 & 576 \\
\hline 6.3 .6 & Particle size & -1.6 & 0 & 46 & 176 \\
\hline
\end{tabular}

\subsubsection{Particle Direction, Applied Bias and Pore Orientation}

Initially, the basic experimental configuration and the concentration polarization mechanism for biphasic pulse generation were investigated. Experiments in this Section used particle set D in $10 \%$ PBS with $V_{0}=-1.6 \mathrm{~V}$. The configuration is defined by the orientation of the pore relative to the sign of the applied bias and the direction of particle motion. The latter was controlled independently of $V_{0}$ by applying pressure using the VPM, and it was ensured that pressure-driven flow was the dominant particle transport mechanism by setting $|P|=0.59-0.98 \mathrm{kPa}\left(6-10 \mathrm{~cm} \mathrm{H}_{2} \mathrm{O}\right)$. The 
most important transport mechanisms for $\sim 200 \mathrm{~nm}$ particles in TRPS are electroosmosis, electrophoresis and pressure-driven flow, and the relative importance of pressure-driven flow to electrophoresis can be calculated using the dimensionless ratio [91]

$$
D_{p d f / e p}=\frac{\eta Q}{\varepsilon \zeta E A}
$$

$Q, E$ and $A$ can be estimated using the previously developed model for transport in TRPS [91] with input parameters $a, b$ and $l$. The pore opening size $a$ can be estimated by linearly scaling $a$ (Table 6.1) with the changing membrane area [82]. Thickness $l$ is determined using an unstretched thickness of $200 \mu \mathrm{m}$ and scaling according to a finite element model of TRPS membrane stretching [78], and a typical value for $b$ is $\sim 30 \mu \mathrm{m}$. The calculation suggests that where $\left|D_{p d f / e p}\right|$ is minimized (at the pore entrance), $\left|D_{p d f / e p}\right|<1$ for $|\zeta|<106 \mathrm{mV}$, well in excess of typical $\zeta$-potentials for carboxylated polystyrene spheres (e.g. -20 to $-50 \mathrm{mV}$ (Chapter 4)). Electroosmosis can be considered by replacing $\zeta$ with the difference between particle and pore $\zeta$-potentials. The latter is typically -10 to $-15 \mathrm{mV}$ (Chapter 5) and the dominance of pressure-driven flow for experiments in this Section 
is unaffected.

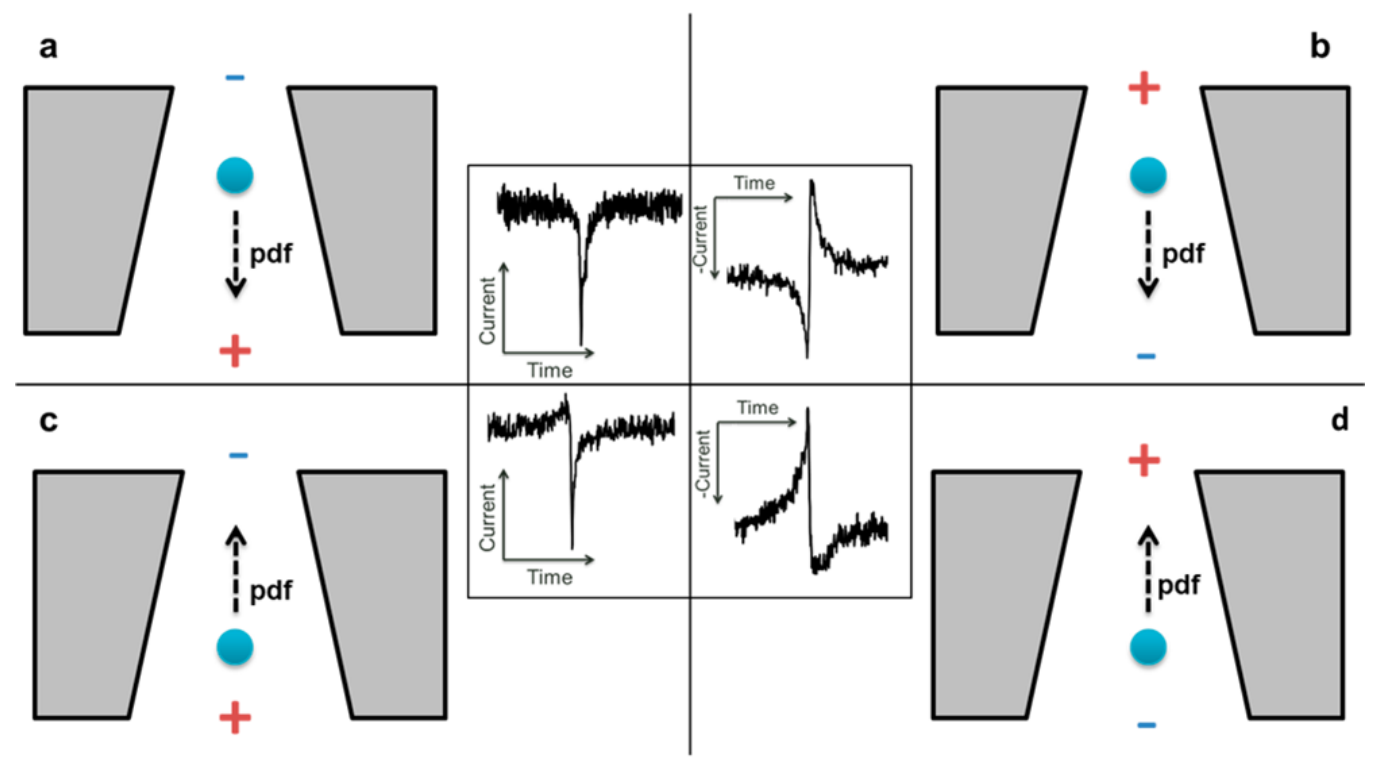

Figure 6.2: Pulse shape dependence on directions of particle motion and $V_{0}$. Each subfigure includes a schematic cross section through the pore and an indicative example of a resistive pulse. (a) Positive $V_{0}$ and positive pressure, as in the conventional TRPS configuration, results in a resistive pulse. (b) Negative $V_{0}$ and positive pressure results in a (conductive, resistive) biphasic pulse. (c) Positive $V_{0}$ and negative pressure results in a (conductive, resistive) biphasic pulse. (d) Negative $V_{0}$ and negative pressure results in a (resistive, conductive) biphasic pulse.

Typical pulses for four configurations of $V_{0}$ and particle motion are shown in Figure 6.2, with the smaller pore facing upwards. Conductive pulses were not detected in the configuration used for conventional TRPS measurements (Figure 6.2a). In Figures 6.2b and 6.2c, a conductive pulse is observed prior to a resistive pulse, and the reverse order is observed in Figure 6.2d. Both normal (small upper opening, large lower opening), 
and opposite (large upper opening, small lower opening) pore orientations were investigated. Experiments with the opposite orientation (i.e. upside-down) did not affect the order and presence of conductive and resistive pulses when the applied bias and direction of particle motion were likewise reversed.

Observations for Figures $6.2 \mathrm{~b}$ to $6.2 \mathrm{~d}$ are consistent with the mechanism for biphasic pulses described by Menestrina et al. [277]. In TRPS, the TPU pore material carries a negative surface charge. In this case, concentration polarization results in a build-up of ions near the cathode and a depletion of ions near the anode (Figure 6.1). When a particle moves through the region of ion enrichment, the overall current is decreased due to volume exclusion (the conventional resistive pulse mechanism) and a reduction of ionic charge carriers within the pore. When the particle moves through a region of depletion near to the anode, counter-ions drawn to the particle increase the availability of carriers within the pore, and the overall current can be enhanced. With this mechanism, a particle travelling through a pore from the cathode to the anode is expected to generate a biphasic pulse in the form of a resistive pulse followed by a conductive pulse (Figure 6.2d). Conversely, when the particle moves from the anode 
to the cathode, the biphasic pulse is a conductive pulse followed by a resistive pulse (Figures $6.2 \mathrm{~b}$ and 6.2c). Inconsistency of the absolute pulse sizes between the different configurations could be caused by variations in $P$, and the asymmetry and specific geometry of the pore.

Lan et al. [87] suggested that conductive pulses were observed when particles were within the ion enrichment region, and were not observed when they passed through a region of ion depletion. While this interpretation is consistent with the experimental and modelling trends in their own work, it does not explain Figure 6.2d, in which the conductive pulse follows the resistive pulse, nor equivalent biphasic pulses observed by Menestrina et al. [277].

In Figure 6.2a only a resistive pulse is observed, consistent with Lan et al.'s result [87] that biphasic pulses disappeared when the applied voltage was switched, changing from a configuration equivalent to Figure $6.2 \mathrm{~b}$ to a configuration equivalent to Figure 6.2a. These observations can be explained by considering two factors. Firstly, observation of a conductive pulse should be aided when the ion-depleted region (hence, the anode) is nearest the most constricted part of the pore (the smaller entrance) where the ionic current is most sensitive to the presence of particle. This is not 
the case in Figures 6.2a and 6.2c. Secondly, the pressure applied in Figures $6.2 \mathrm{a}$ and $6.2 \mathrm{~b}$ acts downwards. It has been suggested [87] that pressuredriven flow rates can change ionic concentration distributions within the pore interior. Figure 6.2a is the only configuration which combines an ion depletion region near the larger pore entrance with a positive applied pressure.

The effects of applied pressure on biphasic pulses were further investigated by varying the negative (upwards) applied pressure over the range 0 to $-13 \mathrm{~cm} \mathrm{H}_{2} \mathrm{O}$ (0 to $-1.3 \mathrm{kPa}$ ) for the same configuration as in Figure 6.2c. Figure 6.3 shows that conductive pulse magnitude $\left(\Delta I_{c}\right)$ becomes larger as a greater negative pressure is applied. Lan et al. found that $\Delta I_{c}$ decreased and eventually disappeared with pressure equivalent to positive (downwards) values from $0.53-4.0 \mathrm{kPa}(4-30 \mathrm{~mm} \mathrm{Hg})$, using a configuration equivalent to Figure $6.2 \mathrm{~b}$ [87]. Both trends are consistent with movement of the ionic depletion region in the direction of the pressure-driven flow. It appears that as the ion depletion region moves closer to the pore constriction, the conductive pulses become larger. This mechanism explains the non-appearance of conductive pulses in Figure 6.2a, and is supported by results from relevant simulations [72] as long as the pressure is not 


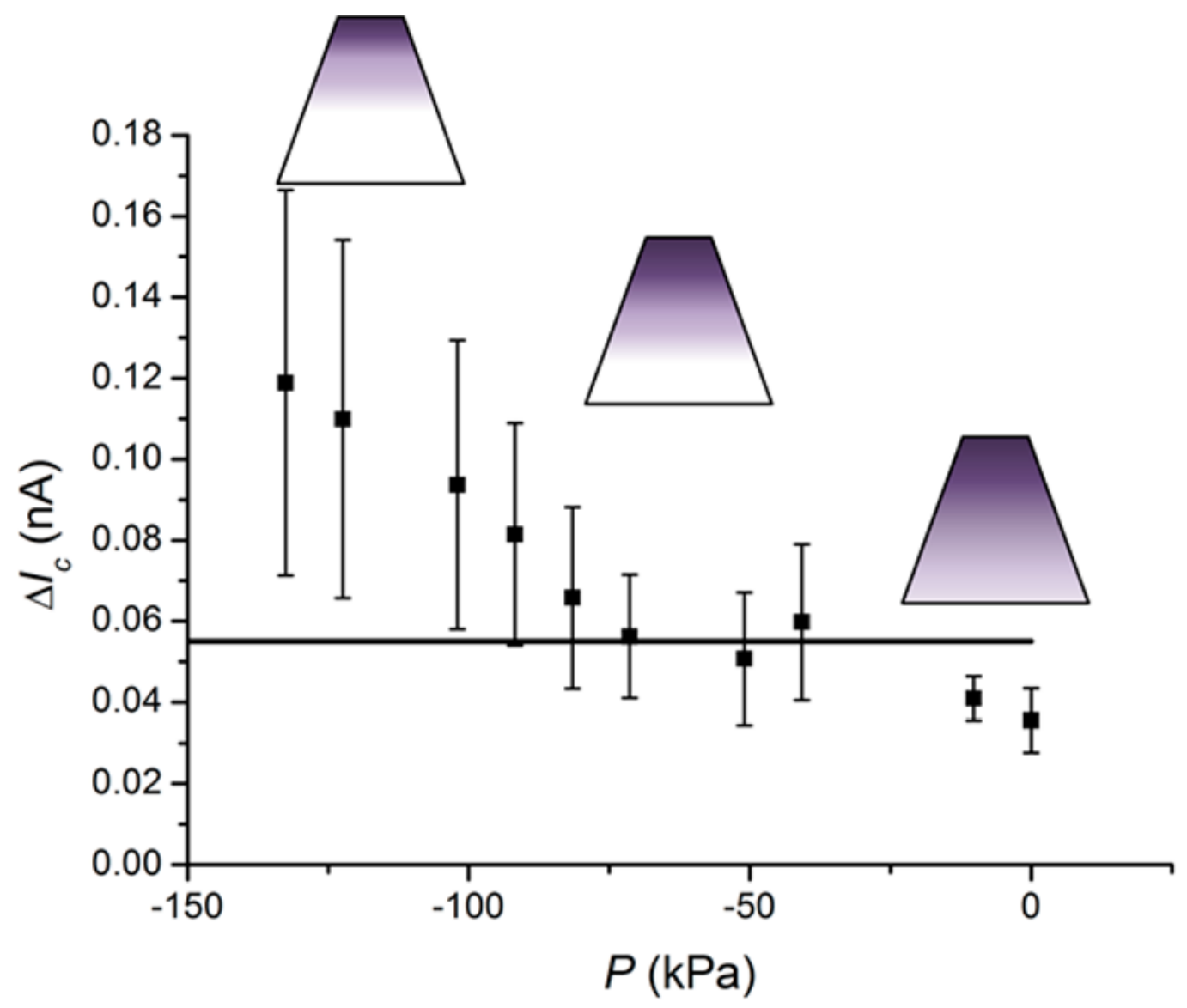

Figure 6.3: Variation of median conductive pulse magnitude $\left(\Delta I_{c}\right)$ with applied negative pressure for experiments in the same configuration as Figure 6.2c. The horizontal line at $0.055 \mathrm{nA}$ indicates the default threshold pulse magnitude, so conductive pulses with magnitudes below the line become difficult to distinguish from the baseline signal. Error bars indicate the upper and lower quartiles. Schematics show the interpretation of the data in terms of the ionic concentration within the conical pore, where darker shading indicates relative ion enrichment. Conductive pulses become more prominent when an ion depleted region (lighter shading) is pushed closer to the pore constriction at relatively large negative pressure. 
large enough to effectively remove variations in ion concentration. Without concentration polarization, a decrease in pulse magnitude at greater absolute pressure may be observed regardless of experimental configuration, because an increase in particle speed can lead to low-pass filtering [74].

\subsubsection{Particle Charge}

The effect of particle surface charge on the size of conductive peaks within a biphasic pulse was investigated using particle sets A-D (Table 3.3) in 10\% PBS. The configuration was the same as in Figure 6.2d, except that particles were driven by electrophoresis $\left(V_{0}=-1.6 \mathrm{~V}\right)$ rather than pressure $(P$ $=0$, see also Section 6.3.4). Conductive pulse magnitude increased monotonically with surface charge (Figure 6.4). Particle set D, which had the highest surface charge density and therefore produced the largest conductive pulses, was used for all further investigations in this Chapter. This increase in $\Delta I_{c}$ with $\sigma$ can be qualitatively explained by considering the EDL surrounding the particle. The extent of the EDL (i.e. the Debye length) is independent of surface charge, taking a value of $\sim 2 \mathrm{~nm}$ for $10 \%$ PBS, and typically 1-5 $\mathrm{nm}$ for the electrolytes used throughout this study. At higher 
surface charge, the density of counter-ions within the EDL increases, so that the mechanism for generating conductive pulses due to concentration polarization (Section 6.3.1) is enhanced. The trend observed in Figure 6.4 is in qualitative agreement with previous computational results and experiments $[87,277]$ in which functionalized particles generated biphasic pulses, but non-functionalized particles produced only standard resistive pulses under the same conditions.

Figure 6.4 suggests that $\Delta I_{c}$ is approximately a linear function of $\sigma$, the nominal surface group density. Rigorous derivation of the quantitative relationship between $\Delta I_{c}$ and EDL charge density is not trivial. However, the postulated conductive pulse mechanism depends on the number of excess ions in the EDL. In typical analyses [279] the total EDL excess charge is equal to $\sigma$, and the local charge density at any point within the EDL is proportional to $\sigma$. It is therefore reasonable to suggest that $\Delta I_{c} \propto \sigma$ as in Figure 6.4. The data point for particle set $D$, which has the greatest value of $\sigma$, lies slightly below the linear fit. This observation could be related to breakdown of the Poisson-Boltzmann model at high surface charge densities. 


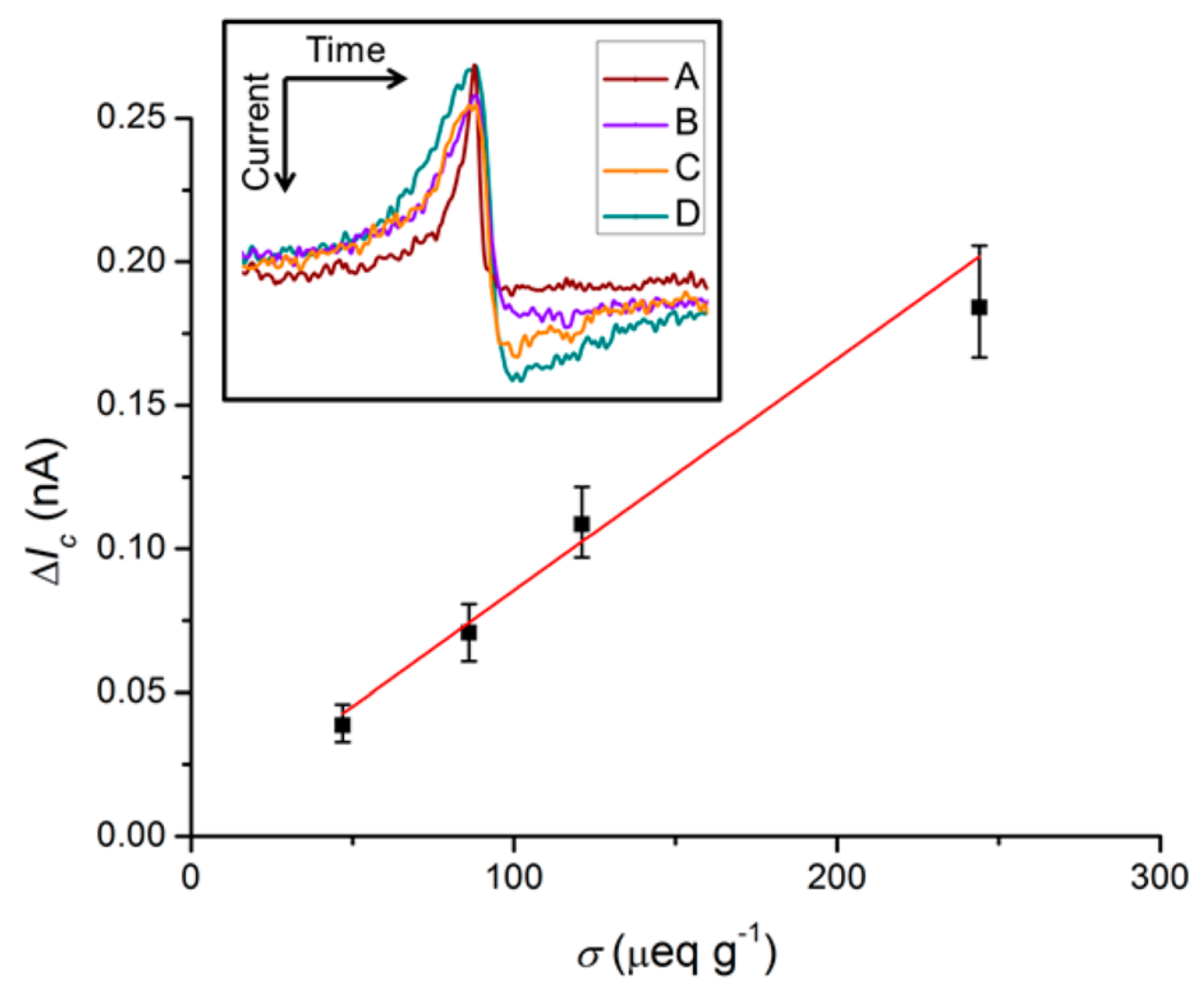

Figure 6.4: Dependence of conductive pulse magnitudes on nominal surface group density for particles in Table 3.3, with a linear fit to the data $\left(\mathrm{R}^{2}\right.$ $=0.987)$. Error bars indicate the upper and lower quartiles. Inset: Example pulses for particles A-D in Table 3.3 under a negative applied voltage, so that the upward pulse is resistive, and the downward pulse is conductive. 


\subsubsection{Electrolyte Concentration}

The effect of electrolyte concentration on biphasic pulses was investigated using particle set $\mathrm{D}$ in a range of PBS dilutions $(100 \%, 75 \%, 50 \%, 25 \%, 10 \%$, and $5 \%)$. It was found that the ratio of $\Delta I_{c}$ to $I_{0}$ increased with decreasing electrolyte concentration (Figure 6.5). At higher electrolyte concentrations, the Debye length is shorter but the total excess charge in the EDL is unchanged. The excess charge at any particular position is

$$
\rho_{e}=e \sum_{i} c_{i} z_{i}
$$

where $e$ is the charge of an electron, and $c_{i}$ and $z_{i}$ are the concentration and valency of ionic species $i$, respectively. For a symmetric 1:1 electrolyte of concentration $C$, the Debye-Hückel and Smoluchowski approximations (respectively valid if the EDL potential is $\leq \sim 25 \mathrm{mV}$ and the EDL is much smaller than the particle size) can be applied to the Poisson-Boltzmann approach to find $\rho_{e}$ at a distance $x$ from a surface (see Equations 5, 7 and 11 in Schoch et al. [90]), 


$$
\rho_{e}(x)=\kappa^{2} e^{-\kappa x}
$$

where $\kappa^{-1}$ is the Debye length, $\kappa^{2} \propto C$. This excess charge density may increase with $C$ for some values of $x$. However, taking the ratio of this charge density to the bulk electrolyte concentration, it is found that

$$
\rho_{e}(x) / C=e^{-c^{0.5} x}
$$

This function suggests that the excess charge relative to the bulk concentration monotonically decreases with $C$ for any value of $x$, consistent with the trend observed in Figure 6.5.

Another analytical approach is to assume that $\Delta I_{c}$ is proportional to the total charge in the EDL (as in Section 6.3.2), and is independent of $C$. If the electrolyte conductivity (hence, $I_{0}$ ) scales linearly with $C$, as expected for a strong electrolyte under ideal conditions [279], then $\Delta I_{c} / I_{0} \propto C^{-1}$. This power law yields a reasonable fit to the experimental data (Figure 6.5), but this approximate analysis does not explain the overall mechanism for conductive pulses. Although the volumetric approach is conceptually 


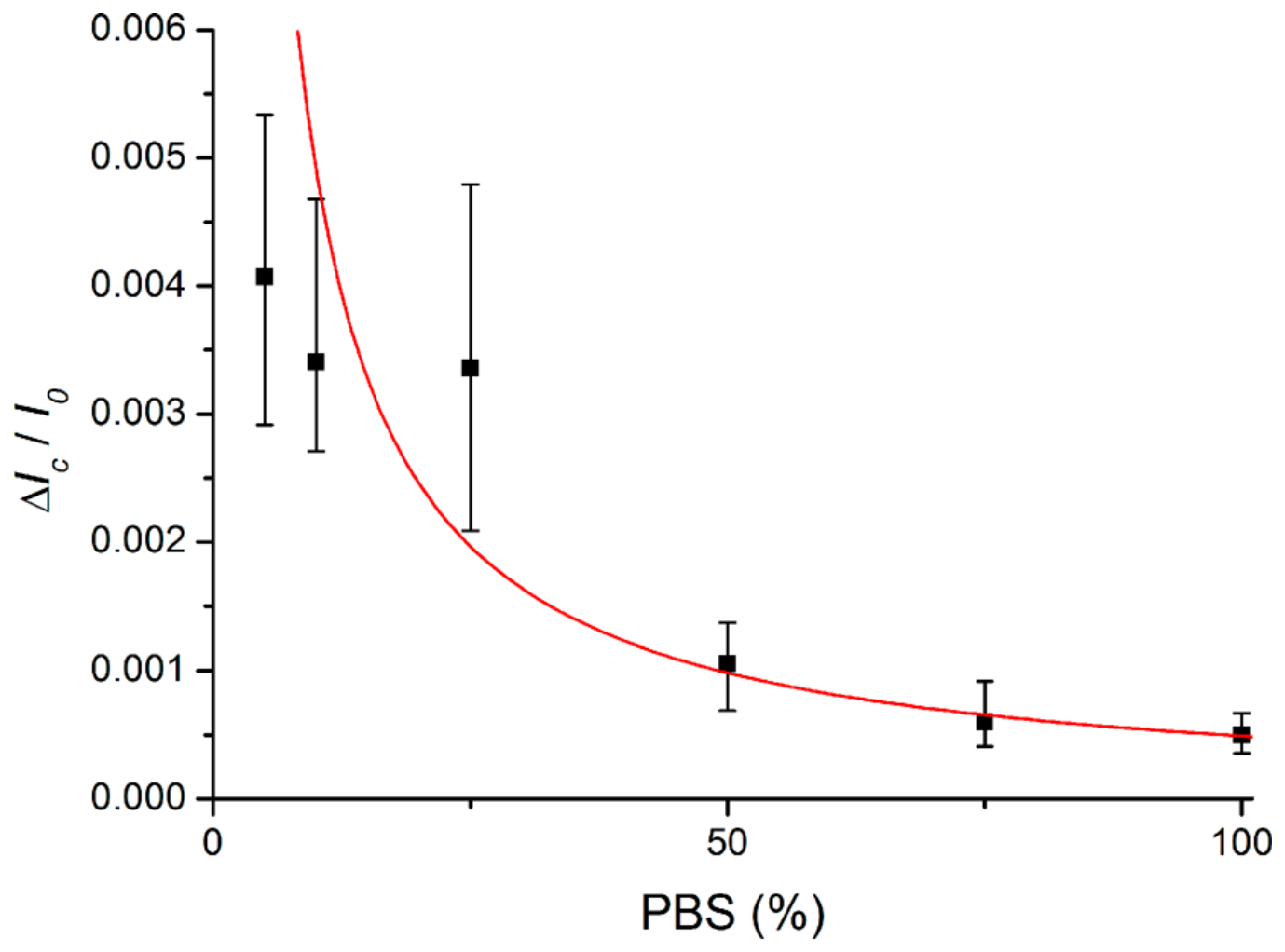

Figure 6.5: Median relative conductive pulse magnitude plotted as a function of electrolyte concentration. Error bars indicate upper and lower quartiles. The line is a power law fit to the data with an exponent of -1 $\left(\mathrm{R}^{2}=0.97\right)$. 
consistent with methods used to analyse resistive pulses, there are differences in the underlying mechanism and assumptions [43]. Additionally, the amount of charge carried in the EDL is insufficient to entirely explain conductive pulses [277].

The onset concentration for conductive pulses is of practical interest. In particular, RPS protocols for measuring particle size and charge are potentially affected by conductive and biphasic pulse phenomena. Figure 6.5 suggests that the size of a conductive pulse crosses the default lower detection threshold $\left(\Delta I_{c} / I_{0}=0.002\right)$ between $25 \%$ and $50 \%$ PBS. Conductive pulses have not been reported previously using TRPS, which is conventionally used with $\sim 100 \mathrm{mM}$ electrolyte.

Because the concentration polarization mechanism depends on pore geometry and materials (among other experimental variables), it is unsurprising that other studies report similar qualitative trends, but with variation in onset values. Biphasic pulses were insignificant at concentrations greater than $300 \mathrm{mM} \mathrm{KCl}$ for Menestrina et al. [277], who recommended using $<200 \mathrm{mM}$ electrolyte for the process of profiling internal pore geometry. Experiments and modelling carried out by Lan et al. [87] suggested that conductive pulses were eliminated by moving from $10 \mathrm{mM}$ to 100 
$\mathrm{mM} \mathrm{KCl}$, and that conductive pulses caused less than a $10 \%$ change in the apparent particle size with less than $0.6 \mathrm{~V}$ applied using $10 \mathrm{mM} \mathrm{KCl}$. Conductive pulses have also been more prevalent at lower electrolyte concentrations in sensing of molecular DNA [268, 269, 271, 272].

\subsubsection{Applied Voltage}

Conductive and resistive pulse magnitudes were measured over a range of applied voltages between -1.6 and $1.6 \mathrm{~V}$ in $10 \%$ PBS with $P=0$. In the range $-0.4 \mathrm{~V}<V_{0}<0.5 \mathrm{~V}$, pulses were small and not reliably distinguished from the background current, so data are not shown. Figure 6.6a shows that when $V_{0}$ was positive, conductive pulses were not detected above the lowest threshold detection level $(0.003 \mathrm{nA})$. This configuration and result are consistent with Figure 6.2a. At negative $V_{0}$, the appearance of biphasic pulses is again dependent on the direction of particle motion, identified by the pulse asymmetry. The onset negative voltage for observation of biphasic pulses $\left(V_{0}=-0.4 \mathrm{~V}\right)$ corresponds to the voltage at which electrophoretic transport dominates pressure-driven flow, so that particles move upward through the pore. This configuration is equivalent to Figure 6.2d, except that pressure acts downward and particle motion in the direction of the 
arrow is driven by electrophoresis. Revisiting Equation 6.1 with the experimental parameters used here (Table 6.1), it is found that at $|V|=-0.4 \mathrm{~V}$, electrophoretic flow dominates $\left(\left|D_{p d f / e p}\right|<1\right)$ as long as the functionalized particles are slightly more charged $(|\zeta|>2.4 \mathrm{mV})$ than the pore surface.

For the biphasic pulses, both $\Delta I_{c}$ and resistive pulse magnitudes $\left(\Delta I_{r}\right)$ increase at greater negative $V_{0}$. The linear fits to the data plotted in Figure 6.6a are supported by the simple volume exclusion mechanism conventionally used for resistive pulses [43], where $\Delta I \propto V_{0}$ when $\Delta I \ll I_{0}$ for an Ohmic pore. The resistive pulses are more sensitive to change in $V_{0}$ than the conductive pulses, as demonstrated by the reduction in the ratio of $\Delta I_{c}$ to $\Delta I_{r}$ with increasing negative $V_{0}$ in Figure 6.6b. This difference between conductive and resistive pulses is not predicted by volume exclusion and reflects the more complicated dependence of the concentration polarization mechanism on $V_{0}$. Surface charge effects are certainly voltage dependent, and equivalence can be drawn with the variations observed in studies of ICR [87].

The trend in Figure 6.6b has not been universally observed. Lan et al.'s [87] modelling results suggested that the ratio of $\Delta I_{c}$ to $\Delta I_{r}$ increased with absolute voltage. Their explanation was that concentration polarization is 

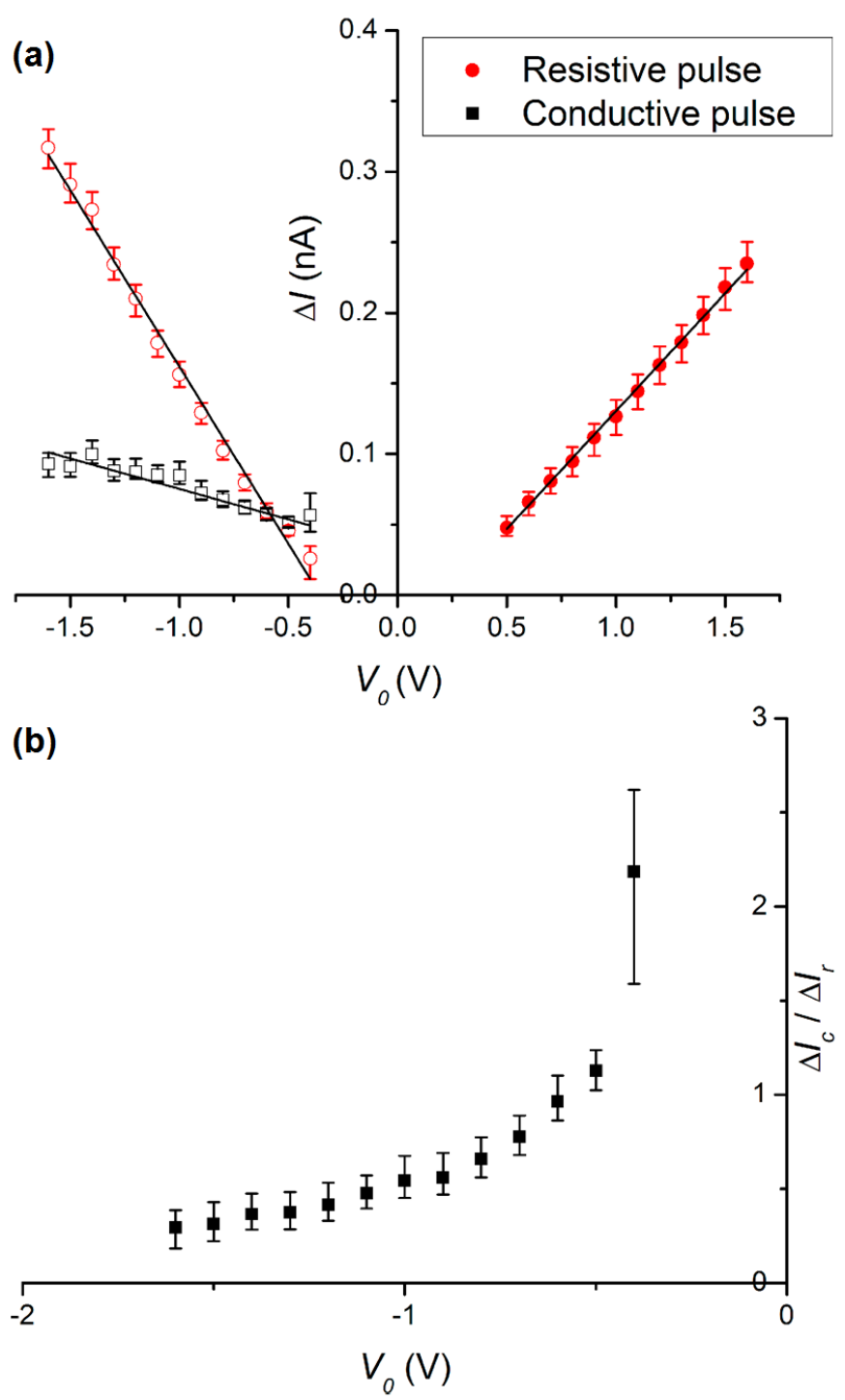

Figure 6.6: Variation of biphasic pulse magnitudes with $V_{0}$. (a) Conductive and resistive pulse magnitudes with $P=0$ in $10 \%$ PBS. Filled markers represent particles travelling in the normal direction (as in Figure 6.2a), open markers represent particles travelling in the opposite direction (as in Figure 6.2d). Lines represent linear fits to the data, with $R^{2}=0.998$ and gradient $=0.167 \mathrm{nA} \mathrm{V}^{-1}$ for $\Delta I_{r}$ at $V_{0}>0, \mathrm{R}^{2}=0.993$, and gradient $=-0.250$ $\mathrm{V}^{-1}$ for $\Delta I_{r}$ at $V_{0}<0$, and $\mathrm{R}^{2}=0.931$ and gradient $=-0.043 \mathrm{nA} \mathrm{V}^{-1}$ for $\Delta I_{c}$ at $V_{0}<0$. Error bars indicate the upper and lower quartiles. (b) Ratio of median conductive and resistive pulse magnitudes from (a). 
enhanced at higher potentials. The modelling was supported by experiments in which biphasic pulses were observed at $-0.4 \mathrm{~V}$ with occasional conductive pulses at $-0.3 \mathrm{~V}$ and none at $-0.2 \mathrm{~V}$ or at positive voltages. Menestrina et al.'s experiments [277] also weakly suggested that the ratio of $\Delta I_{c}$ to $\Delta I_{r}$ increased with $V_{0}$ up to $4 \mathrm{~V}$. Interesting voltage dependencies have been reported elsewhere, including observation of biphasic pulses only at intermediate potentials for dsDNA [271] and dependence of $\Delta I_{c} / I_{0}$ on the sign of $V_{0}$ for $20 \mathrm{~nm}$ gold nanoparticles [275].

\subsubsection{Applied Stretch}

Experiments were carried out with the stretch applied to a membrane varied in a stepwise manner, with results shown in Figure 6.7. Both resistive and conductive pulse magnitudes monotonically decrease as the stretch increases. The decrease in absolute $\Delta I_{r}$ with increasing pore diameter is expected based on the volume exclusion approach [43], in which the resistive pulse magnitude is proportional to $A^{-2}$.

It is qualitatively reasonable that $\Delta I_{c}$ should also decrease with increasing pore size, as the charge carried by the particle becomes less significant 

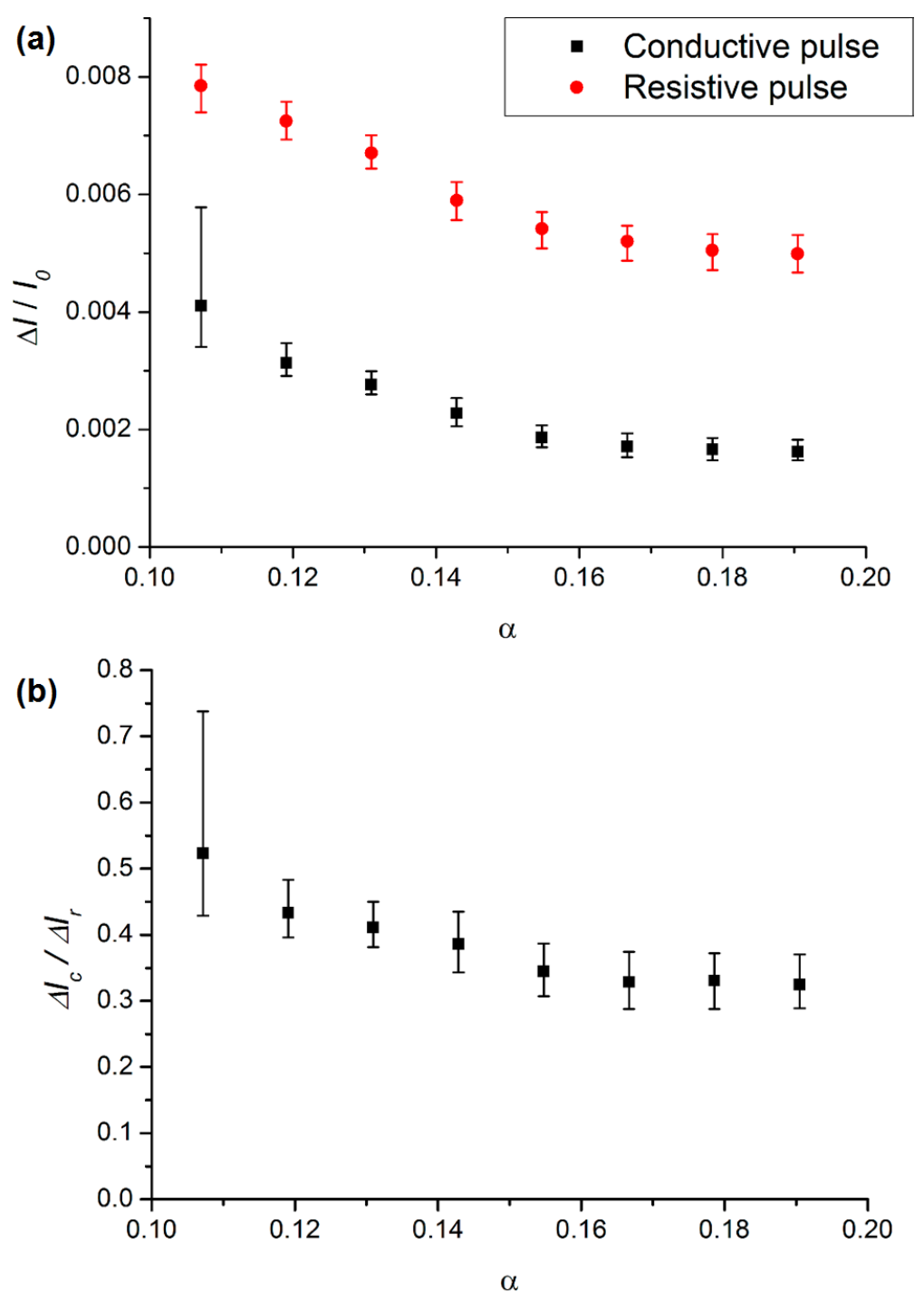

Figure 6.7: Variation of biphasic pulse magnitudes with $\alpha$, the macroscopic strain $\left(\alpha=\left(X-X_{0}\right) / X_{0}\right)$, and therefore pore size. (a) Conductive and resistive pulse magnitudes in 10\% PBS, with $V_{0}=-0.7 \mathrm{~V}$ and $P=0$. (b) Ratio of median conductive and resistive pulse magnitudes from (a). Error bars indicate the upper and lower quartiles. 
relative to the overall ionic distribution. However, the nonequivalence of resistive and conductive pulse mechanisms is again evident, because $\Delta I_{c}$ decreases more quickly with applied stretch than $\Delta I_{r}$ (Figure 6.7b). Consistent with this result, Menestrina et al. [277] found that conductive pulses were less prominent for a relatively large pore. Equivalently, larger particles measured using the same pore produced higher $\Delta I_{c} / \Delta I_{r}$ ratios for $470 \mathrm{~nm}$ particles than for $410 \mathrm{~nm}$ particles, a trend that has also been modeled [87]. However, further experimental data [277] suggested the opposite trend when comparing 280 and $410 \mathrm{~nm}$ particles. As with resistive pulses, conductive pulses can be tuned and optimized in situ using TRPS. This process could be an important tool in further experimental exploration of the relationship between particle surface charge, pore geometry, electrolytes, and the resulting resistive and conductive pulses.

\subsubsection{Particle Sizing}

The discovery of biphasic pulses in TRPS brings to light concerns relating to the accuracy of particle sizing when there is a difference in charge between the calibration and sample particles, which will often be the case. Although in a biphasic pulse the resistive and conductive components ap- 
pear somewhat separated, there is inevitably some overlap and it is expected that under conditions where a conductive pulse is observed, the resistive pulse will be reduced from its total value and its magnitude will not be representative of the total particle volume.

Table 6.2 shows sizing data for particles A-D when sized in 100\% PBS, where biphasic pulses are not observed, and 10\% PBS, where biphasic pulses are observed. Both sizing experiments were calibrated with neutral particles which do not give biphasic pulses. It can be seen that for particle sets A-C there is a reduction in measured particle diameter at the lower electrolyte concentration. This is expected due to overlap of the conductive pulse with the resistive pulse. Particle set D sees very little change in particle diameter between electrolyte concentration. Future work here could better understand these results and the effect of biphasic pulses on particle sizing more generally.

Table 6.2: Particles sized using TRPS with and without visible biphasic pulses.

\begin{tabular}{|c|c|c|}
\hline Particle set & Diameter at 100\% PBS (nm) & Diameter at 10\% PBS (nm) \\
\hline A & $213 \pm 2.5$ & $182 \pm 2.5$ \\
\hline B & $213 \pm 2.5$ & $163 \pm 2.5$ \\
\hline C & $188 \pm 2.5$ & $168 \pm 2.5$ \\
\hline D & $173 \pm 2.5$ & $178 \pm 2.5$ \\
\hline
\end{tabular}




\subsection{Conclusion}

In this Chapter TRPS has been used to study biphasic pulses, in particular their conductive component, as a function of several quantified experimental variables. The chronological ordering of each biphasic pulse is consistent with concentration polarization, where the conductive component is generated as the particle passes through the ion depletion region. Results obtained at varied applied pressure indicate that the ionic depletion region can be shifted in the direction of flow. A quantitative link between conductive pulse magnitude and excess charge in the EDL is suggested by the linear relation between conductive pulse magnitude and particle surface group density. Conductive pulse magnitude monotonically decreases with increasing electrolyte concentration, and the onset concentration is $\sim 50 \mathrm{mM}$. The ratio of conductive to resistive pulse magnitudes decreases with increases in both negative potential and pore size, indicating a difference in mechanisms between conductive and resistive pulses.

This work will be important for TRPS users, especially if operating below conventional (or physiological) salt concentrations of $\sim 100 \mathrm{mM}$. Many of the observed trends will extend to other RPS techniques operating at 
similar length scales, although specific materials and pore geometries affect quantitative results. The size-tunable function of TRPS suggests further experiments which could contribute to the fundamental understanding of conductive and biphasic pulses. Also, the demonstrated relationship between biphasic pulses and particle surface charge suggests a possible method for surface charge measurement, as well as applications in particle discrimination and monitoring of on-bead chemistry. 


\section{Chapter 7}

\section{Broadening of Pulse Size}

\section{Distributions}

In this Chapter, the breadth of pulse size distributions is studied using nominally monodisperse particle size standards. The TRPS sizing method [43] that has been established and validated alongside EM [12, 43, 124] uses the simple relation that the pulse magnitude $(\Delta I)$ is proportional to particle volume. When using this approach, it is assumed that each pulse accurately measures the size of a single particle, so that the breadth of a pulse distribution is determined solely by the size dispersity of the mea- 
sured particle set. However, pulse size distributions can be affected by other factors, some of which are known and can be mitigated, such as insufficient sampling frequency [51, 185], detection thresholds [12, 177], and reduction of resistive pulse heights for highly charged particles in low molarity electrolytes [236]. Here, the initial hypothesis is that off-axis transport is primarily responsible for the distribution broadening.

The effects of particle trajectory have not been thoroughly studied using TRPS. Trajectory variation could add random uncertainty to the size of any individual pulse, broadening the pulse size distribution. Understanding and reducing uncertainty represents important progress for nanoparticle size measurements, which can be inconsistent between different measurement techniques $[12,14,55,103,124]$. It has previously been shown that the resistive pulse size increases with the distance of the particle from the central axis of the pore, using theory and simulations [280-282] as well as other experimental systems [282-284]. Smythe et al. [280] first studied this effect analytically for a long cylindrical pore without end effects, and without considering surface charge. Berge et al. [283] experimentally studied resistive pulses generated by $15 \mu \mathrm{m}$ spheres passing through cylindrical pores, which suggested that differences in pulse magnitude $(<10 \%)$ 
were overestimated by Smythe et al.'s formula, and provided a correlation consistent with their results. Saleh and Sohn [284] studied $\sim 500 \mathrm{~nm}$ latex colloids, and suggested an empirically-derived correction to Berge et al.'s correlation to account for particle trajectory. Qin et al. [281] used FEM of a cylindrical pore to propose a new correlation predicting the resistance change caused by off-axis particles. Most recently, Tsutsui et al. [282] used FEM alongside experiments to study 510-900 nm particles travelling through cylindrical SiN pores with low aspect ratios of membrane thickness to pore width $(\leq 1)$.

The usual output of a TRPS experiment is a histogram of pulse magnitudes (Figure 7.1a), approximated as a normal distribution for statistical analysis (although TRPS distributions are often positively skewed). If the experiment has been calibrated, a distribution of particle sizes can be obtained, where the particle volume is proportional to the pulse magnitude [43]. Therefore after taking the cube root of TRPS pulse magnitudes, the resulting distribution is equivalent to a linear size measurement (i.e. particle diameter), which is usually of most interest for a sizing experiment.

Such TRPS distributions can be directly compared with similar measurements using other techniques. Figure $7.1 \mathrm{~b}$ shows that size distribu- 

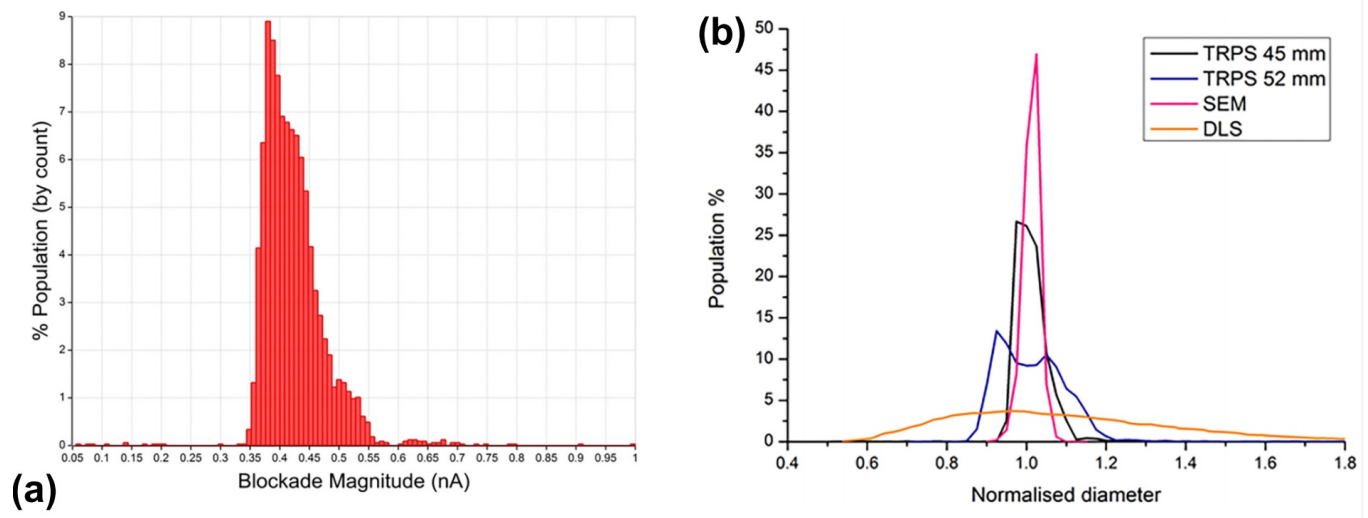

Figure 7.1: Typical size distribution measurements. Figure 7.1a is a TRPS blockade magnitude histogram obtained using particle set $\mathrm{B}$ and pore 1 with $45 \mathrm{~mm}$ applied stretch. Figure $7.1 \mathrm{~b}$ compares size distributions for the same pore and particles using TRPS at two stretch settings, SEM, and DLS. Each data set is normalised by the mean value, and distributed into histogram bins of width 0.025 on the normalised scale.

tions vary between techniques, even for nominally monodisperse size standard particles. EM, here SEM, is considered a gold standard in terms of accuracy. The biggest drawback of EM remains the time required for sample preparation and image analysis, and there may be some uncertainty regarding the effects of sample preparation and sampling strategy for size distributions. The DLS data are very broadly distributed. DLS size distributions are determined using light scattering data, which is dominated by relatively large particles [14, 177]. TRPS distributions are seen to broaden as the size of the pore opening is increased, and eventually two peaks can be observed. To quantify the width of these distributions for comparison 
with independent measurements of particle diameter, the CV is used, corresponding to the linear particle size measurement.

Aside from the specific technology and materials used, experiments in this Chapter build upon these previous studies because they systematically step through changes in the pore size using the elastomeric function of TRPS. Double peaks in size distributions, which have not been addressed previously, are measured and explained. As in some previous work $[283,284]$, the most important transport mechanism in these experiments is pressure-driven flow and therefore the effect of varying the applied pressure is investigated. Applied voltage and pore orientation are also varied.

Results are presented alongside data obtained using pressure reversal experiments in which the same particle is repeatedly passed back and forth through the pore by changing the direction of the applied pressure (see Section 7.1). These are advantageous to consider they generate a pulse distribution that is independent of size dispersity. FEM data also shown help with understanding particle trajectories through a pore, and their influence on the resistive pulses generated. Calculated trajectories are used in order to better understand the expected size distribution of pulses gen- 
erated by monodisperse particles.

Much of the material in this Chapter has been published in [285]. All of the TRPS experiments excluding the single particle reverse pressure experiments and the data in Figure 7.9 were performed by Weatherall. The data in Figure 7.9 were obtained by Vogel and analysed by Weatherall. The FEM and analysis was done by Hauer and Willmott. Willmott provided discussion and suggestions on the writing style and content of the paper.

\subsection{Reference Data}

\subsubsection{Finite Element Modelling of Particle Trajectories}

The modelling referenced in this Chapter was carried out using COMSOL Multiphysics v.4.4 by Hauer [285]. The geometry was defined as a regular truncated cone adjoining the two reservoirs of the fluid cell (Figure 7.2) with $a=5.0 \mu \mathrm{m}, b=50 \mu \mathrm{m}, l=150 \mu \mathrm{m}$, and $d=1.0 \mu \mathrm{m}$. The applied potential was $V_{0}=-0.1 \mathrm{~V}$, and the pressure was $P_{t o t}=980 \mathrm{~Pa}$. The aim of this modelling was to complement the experiments presented in this Chapter by explaining the mechanism for broadening of pulse distributions and 

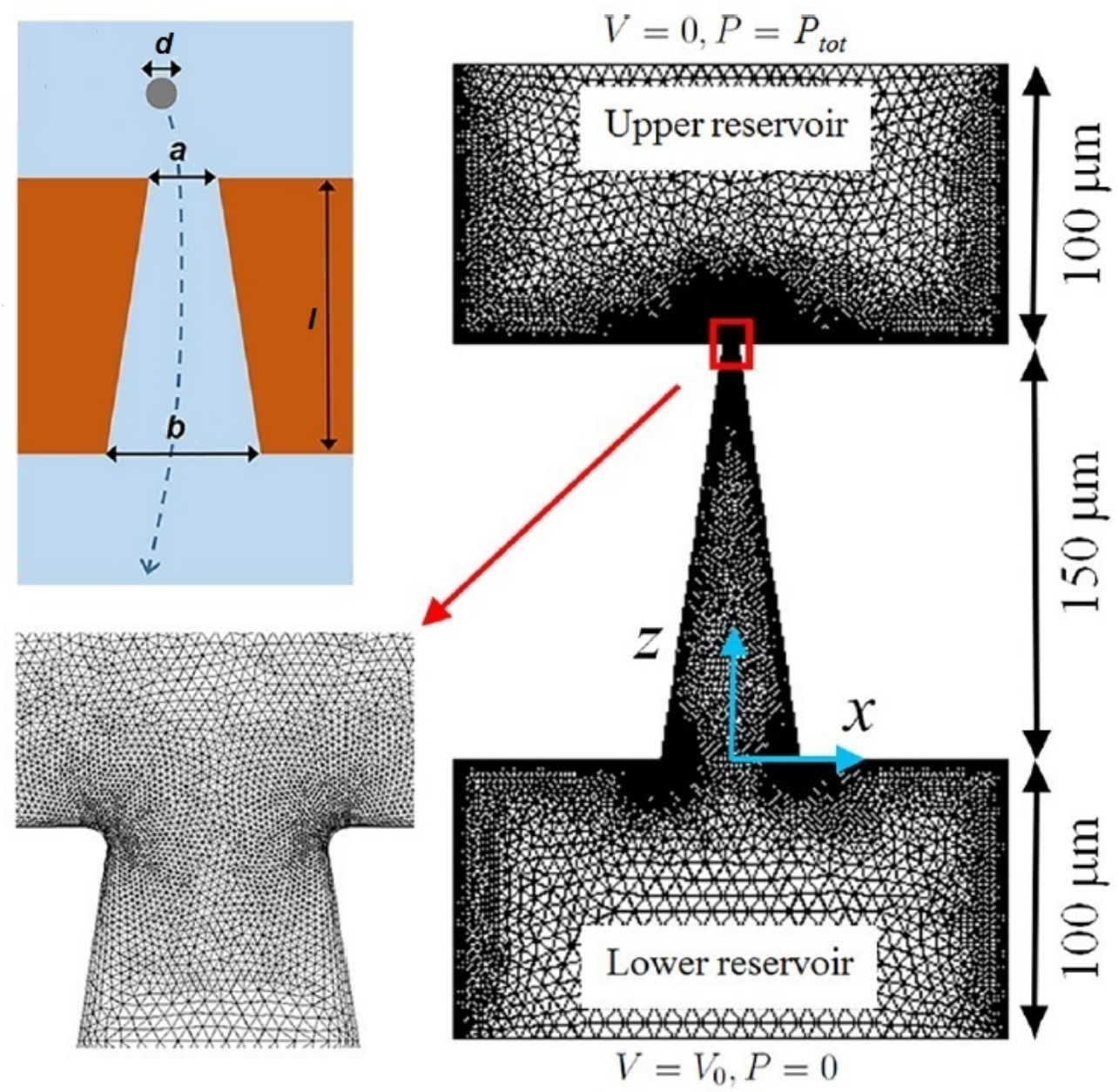

Figure 7.2: Geometry used for modelling. Upper left, schematic crosssection through a conical pore with entrance diameters $a$ and $b$, and membrane thickness $l$. A spherical particle follows some trajectory (dashed line) through the pore to generate a resistive pulse. Right, cross section for the model, including co-ordinate axes (blue). A magnification of the red rectangle (lower left) shows the meshing and rounded edges near the smaller pore entrance. Reproduced from [285]. 
the appearance of two peaks by accounting for the particle trajectories.

Particle trajectories were modeled with pressure-driven flow as the dominant transport mechanism. To include inertial effects and therefore hydrodynamic focusing [286, 287], an FEM arbitrary Lagrangian Euler method (denoted ALE) was first implemented in 2D.

It was found that particles with trajectories near the central axis have relatively small pulse magnitudes and short durations (Figure 7.3a) and particles passing through the region of enhanced electric field near the pore edge produce pulses with larger magnitudes and durations (Figure 7.3a, inset). To investigate whether hydrodynamic focusing has a noticeable effect ALE particle trajectories were then compared with a point particle trajectories where the particle simply moves along stream lines (Figure 7.3b). ALE particle trajectories deviate slightly toward the center of the pore at its narrowest point. Therefore, it is expected that the pulse magnitude distribution should shift to slightly lower values of $\Delta I$ when hydrodynamic focusing is included, although this effect is relatively minor in the presented experiments (Section 7.2.2).

A 3D model was used to obtain a distribution of $\Delta I$ values due to vari- 

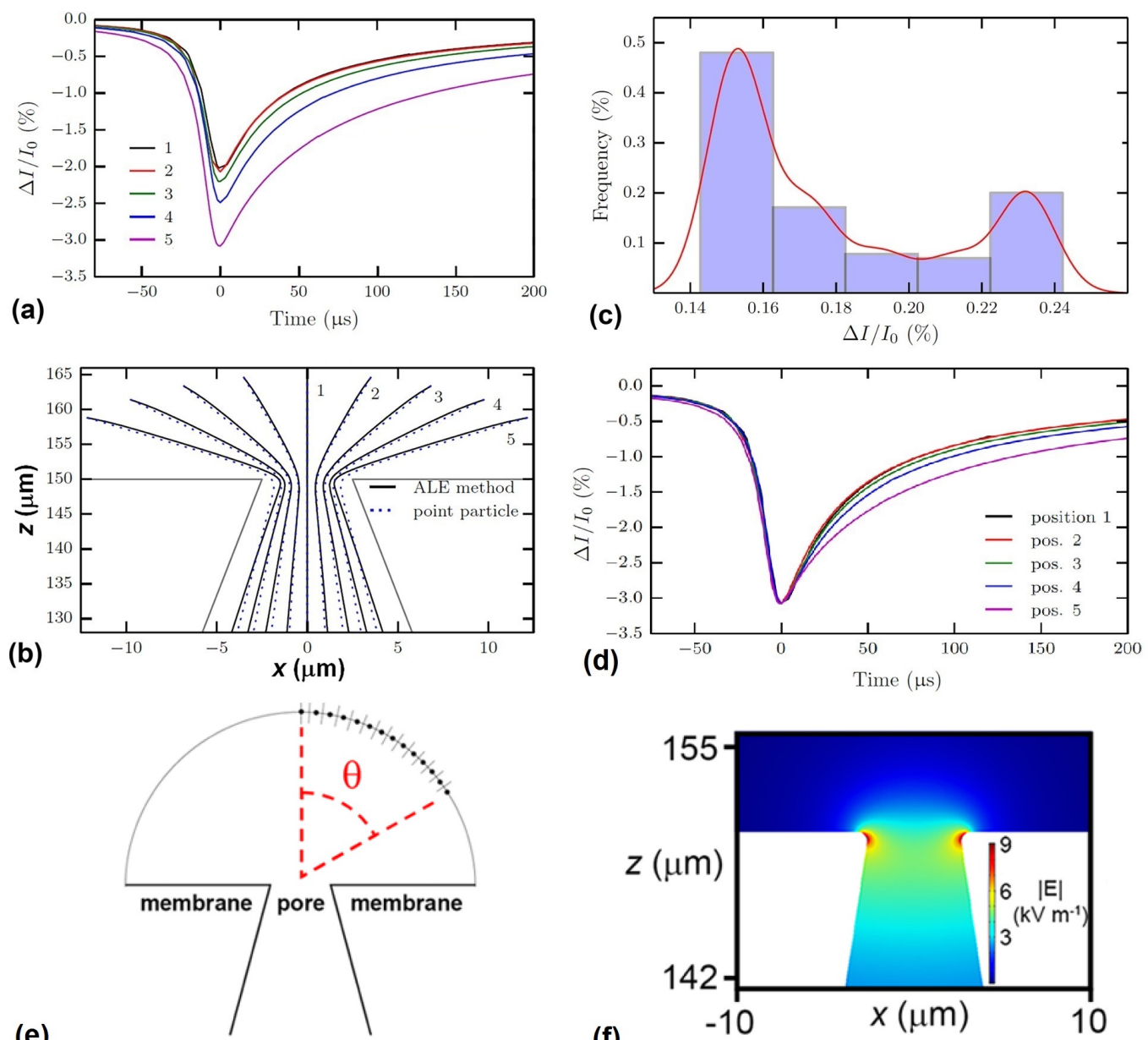

(e)

(f)

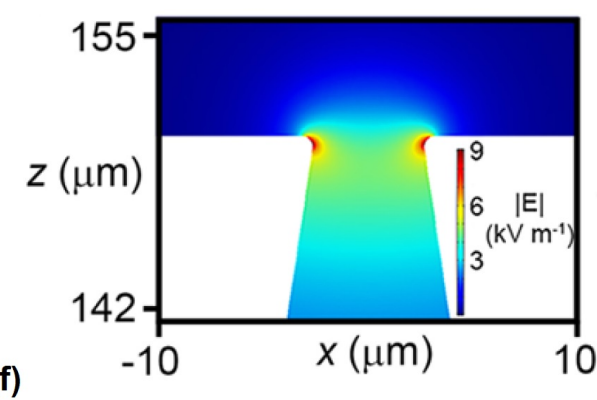

Figure 7.3: FEM resistive pulse simulations. (a) 2D resistive pulses simulated using the arbitrary Lagrangian Euler (ALE) method for five particle trajectories corresponding to different starting positions. The numbered trajectories are depicted in (b), which compares 2D trajectories using the ALE and point particle methods. (c) Shows the resistive pulse size distribution (bin width $0.02 \%$ ) calculated using 3D point particle simulations for 14 starting positions (e). The red line is constructed by adding Gaussian functions centred on each histogram bin. (d) The simulated pulses from (a) are re-plotted with normalised magnitudes. (f) Intensity map of the electric field magnitude near the pore entrance with no particle present (3D simulation). Reproduced from [285]. 
ation in trajectories. The resulting pulse size distribution has two peaks. The peak at higher values of $\Delta I$ can be attributed to the large proportion of particles approaching the pore at relatively large angles. Above a particular initial angle all particles take a trajectory so that they hit the membrane surface. In the model, it is assumed that all such particles enter the pore at the edge, generating relatively large pulses. The peak at lower values of $\Delta I$ is formed because the resistive pulse magnitude does not greatly vary over most of the trajectories in Figure 7.3a.

These results are broadly consistent with previous studies using resistive pulse sensors with different geometries, which suggest that off-axis particles result in pulses with larger magnitudes [281, 283, 288].

\subsubsection{Single Particle Pressure Reversal}

Pulse size distributions have been obtained for single particles as they move back and forth through tunable pores. The direction of the pressuredriven flow applied to the fluid cell was alternated. Each reversal was triggered by observation of a resistive pulse.

Pulse magnitude distributions were measured for individual $400 \mathrm{~nm}$ 

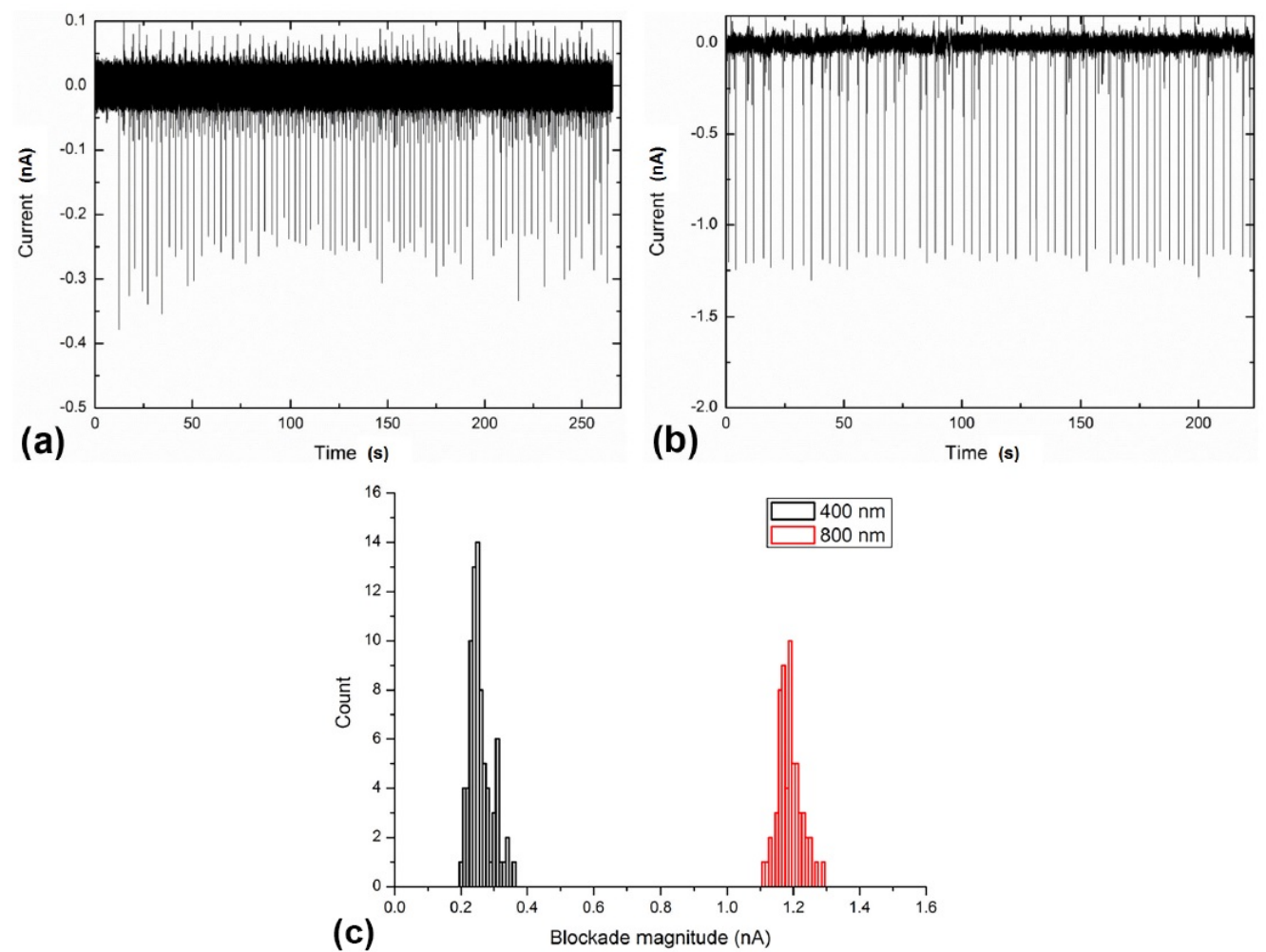

Figure 7.4: Examples of TRPS pulses for single particles moving back and forth through a pore due to applied pressure alternating between $\pm 6 \mathrm{~cm}$ $\mathrm{H}_{2} \mathrm{O}$. Pore 5 was used at $X=45.8 \mathrm{~mm}$ stretch, and $V_{0}=0.32 \mathrm{~V}$. Figure 7.4a: 79 pulses for a $400 \mathrm{~nm}$ particles. Figure 7.4b: 60 blockades for an $800 \mathrm{~nm}$ particle. Figure 7.4c compares pulse histograms for both particles. 
(Figure 7.4a) and $800 \mathrm{~nm}$ (Figure 7.4b) particles in this way. These data were all obtained using an NP400 pore at the same stretch and voltage conditions. There was less variation in blockade magnitude for larger particles relative to $I_{0}$, as the pulse distribution CVs for $400 \mathrm{~nm}$ particles and $800 \mathrm{~nm}$ particles were $4.4 \%$ and $1.0 \%$ respectively (Figure $7.4 \mathrm{c}$ ). These data sets for individual particles remove complications associated with particle dispersity, because all of the pulses are generated by the same particle.

Distribution broadening is a relatively small effect here because a particle's trajectory is correlated from one pulse to the next. In Figure 7.4a the pulses prior to $50 \mathrm{~s}$ ( $\sim 13$ reversals) after the start of the experiment are relatively large compared to those that occur afterwards. This is consistent with an initial period of focussing in which a particle moves sideways $\sim$ $0.1 \mu \mathrm{m}$ during each traversal (see Figure 7.3a). In comparison Berge et al. [283], using $2 \mathrm{~Hz}$ pressure reversals with long (aspect ratio $~ 10$ ) cylindrical pores and $15 \mu \mathrm{m}$ particles, found that the particles came to an (off-axis) equilibrium position after 20-80 reversals.

The non-monotonic variation in pulse heights suggests that Brownian motion has a considerable influence on the particle position over the timescale of the pressure reversals. The distribution CV for $800 \mathrm{~nm}$ particles 
is considerably smaller than for the $400 \mathrm{~nm}$ particles, even though the same pore was used at the same stretch settings for both particle sets. This suggests that the size of pulses generated by the $800 \mathrm{~nm}$ particles is less strongly dependent on trajectory.

\subsection{Results and Discussion}

TRPS distributions broaden as the size of the pore opening is increased, and eventually two peaks can be observed (Figure 7.1b). The complementary FEM studies (Section 7.1) suggest that the peak at lower $\Delta I$ is formed because the resistive pulse magnitude does not vary greatly over small angle trajectories that lead particles close to the centre of the pore. The peak at higher $\Delta I$ is attributed to large angle trajectories that lead particles to the membrane surface and therefore entering at the pore edge. In the following Sections detailed experimental results are presented for particle populations with the variation of stretch, pressure and voltage. Particles used in this Chapter are summarised in Table 7.1. The electrolyte used for all measurements was SEB. 
Table 7.1: Polystyrene particle sets used, including CVs in the diameter. ${ }^{a}$ CVs from SEM measurements. ${ }^{b}$ XPR 1961 series, with CVs provided by the supplier.

\begin{tabular}{|c|c|c|c|c|}
\hline Label & Nominal diameter & Supplier & CV & CV (DLS) \\
\hline B & 220 & Bangs Laboratories & $1.9 \%^{a}$ & $25.3 \%$ \\
\hline L & 350 & Polysciences & $2.5 \%^{a}$ & $24.4 \%$ \\
\hline M & 220 & Thermo-Fisher & $<5 \%^{b}$ & - \\
\hline
\end{tabular}

\subsubsection{Stretch Variation}

A pore at a particular stretch can measure a finite range of particle sizes, based on its diameter. When particles are too small, they can pass undetected due to the electronic noise (or particles passing near the pore without passing through [282]) in the measured ionic current. In the experiments reported in this Chapter, the smallest particles detected give a blockade magnitude above the threshold of the instrument, so the lower size detection threshold is not important. The upper size limit is determined by whether or not particles can pass through the pore unhindered. In order to fully characterise a sample, the user may need to use multiple stretches and even multiple pores [77].

Figures 7.5 and 7.6 demonstrate variation in the normalised pulse size distribution for particle set B (nominally, monodisperse $200 \mathrm{~nm}$ particles) on pores 1 and 2, and for particle set L (nominally, monodisperse 400 

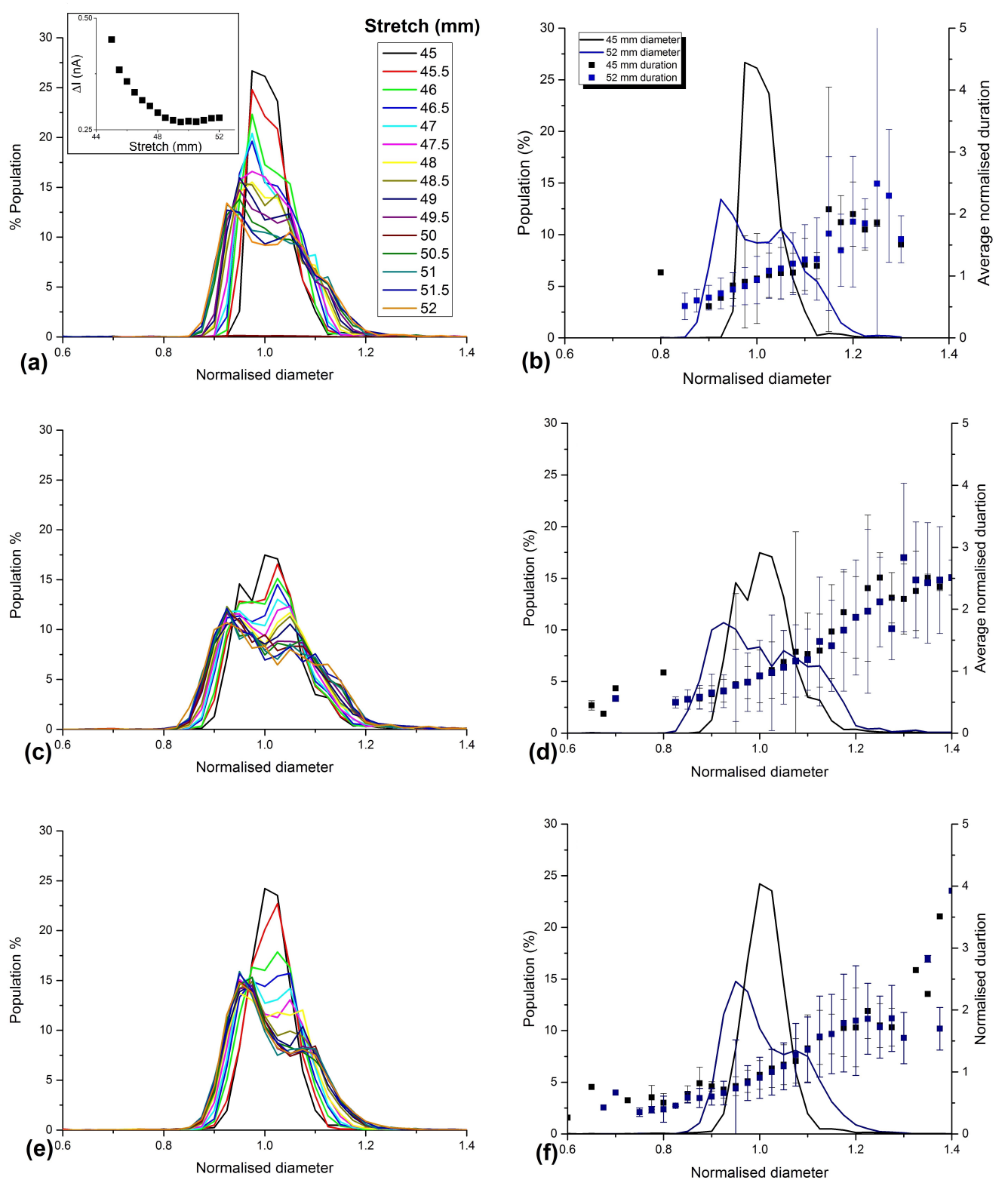

Figure 7.5: TRPS pulse size distributions as a function of stretch for (a) particle set B pore 1 (c) particle set B pore 2 and (e) particle set L pore 3. In Figures 7.5b, $\mathrm{d}$ and $\mathrm{f}$, size histograms from Figures 7.5a, c and e respectively are plotted at two stretches, along with the average baseline duration for pulses in each histogram bin. Error bars indicate the standard deviation, not shown for single events. Durations are normalised by the average for all data. Pore and particle set details can be found in Table 3.2 and Table 7.1 respectively. 
$\mathrm{nm}$ particles) on pore 3 as a function of membrane stretch. The conventional TRPS method for determining particle size assumes that $\Delta I$ is proportional to the particle volume, so the $\mathrm{CV}$ is calculated using the cube roots of TRPS pulse magnitudes. CVs of whole distributions were determined by dividing the standard deviation for all blockades by the mean for all blockades. It was found (Figures 7.5 and 7.6a) that the widths of normalised pulse magnitude distributions increase with stretch, so that the calculated particle size distributions would similarly broaden. For the same particle sets, size distributions independently measured using SEM (Table 7.1) yielded CVs lower than those plotted in Figure 7.6a, even at low stretch settings. Saleh and Sohn [284] similarly found that their distribution width was not explained by particle size dispersity, as the range of dwell times (80\%) was much larger than the predicted range $(2 \%)$ based on the manufacturer specification of particle size. The mean absolute pulse size (Figure 7.5a inset) decreased as the particle to pore size ratio decreased with stretch, as expected for resistive pulses [19, 236].

At high stretches, the shape of the distribution changes in addition to the width, and a second peak becomes apparent (Figure 7.5a, c and e). If the distribution is fitted with two peaks (using OriginPro 8 software), then 

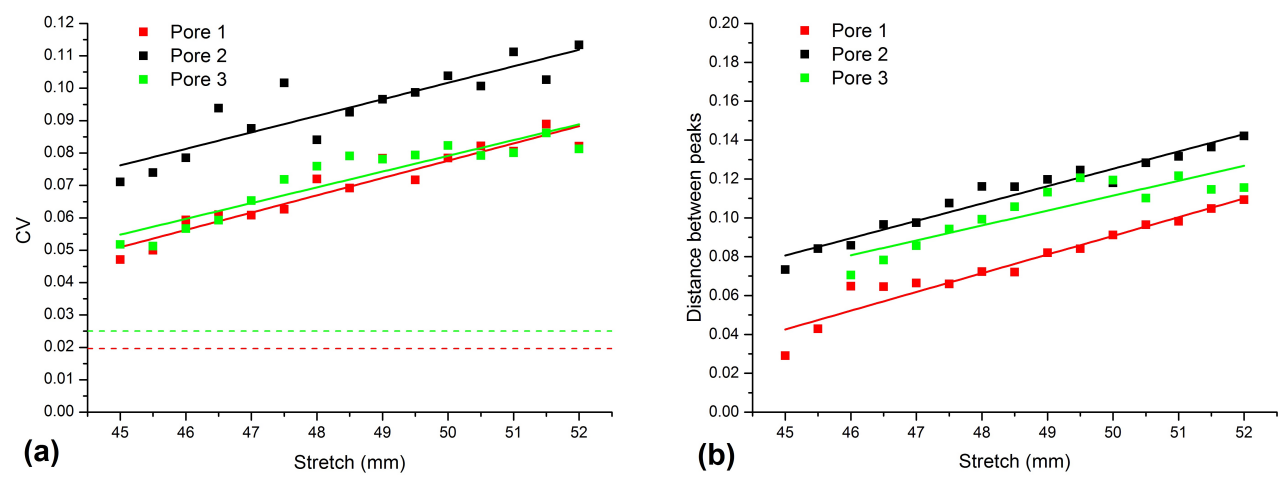

Figure 7.6: (a) Size distribution CVs using pores 1, 2 (both particle set B, $V_{0}=0.32 \mathrm{~V}$ ) and 3 (particle set $\mathrm{L}, V_{0}=0.22 \mathrm{~V}$ ). Dashed lines indicate CVs from SEM for particle sets A (red) and B (green). Other lines are linear fits to each data set. (b) Separation of peaks from Figure 7.5a (normalised diameter units) when two peaks are fitted to each distribution. Lines are linear fits to each data set. Pore and particle set details can be found in Table 3.2 and Table 7.1 respectively.

the distance between these peaks becomes larger as the stretch increases (Figure 7.6b). Assuming that all of the particles in the sample are the same size, the presence of two peaks indicates two favoured types of trajectory, as seen in simulations. As the pore is stretched, it appears that the difference in resistive pulse sizes between a particle moving on-axis and a particle near the edge of the pore increases.

The observations in Figures 7.5 and 7.6 suggest that the difference between extreme $\Delta I / I_{0}$ values increases with pore size. This is consistent with the decrease in $\mathrm{CV}$ seen with decreasing particle to pore size ratio in 
pressure reversal experiements. In attempting to explain this trend, the geometric parameters of pore 1 were estimated using measurements of $R_{0}$ and Equation 2.8. A resting thickness of $210 \mu \mathrm{m}$ at $41.5 \mathrm{~mm}$ stretch [99] and scaling according to a hyperelastic material model [78] is assumed. The larger pore diameter $b$ is determined from the equivalent diameter from SEM imaging at $45 \mathrm{~mm}$ stretch $(23.2 \mu \mathrm{m})$, and $a$ and $b$ are assumed to scale proportionally to each other. Using this approach, values of $a=1.0$ $\mu \mathrm{m}$ and $1.6 \mu \mathrm{m}$ were obtained for stretches of 45 and $52 \mathrm{~mm}$ respectively a $58 \%$ increase.

Using previous models for cylindrical pores, these values of $a$ can be used as the pore diameter in order to estimate the effect of changing pore size on pulse sizes. Qin et al.'s approach [281] produces a very slight decrease in the range of normalised pulse sizes between $45 \mathrm{~mm}$ and $52 \mathrm{~mm}$ stretch $(0.05 \%$, opposite to our experimental trend). Using Berge et al.'s approach [283] the normalized pulse size range also decreases from $2.1 \%$ to $0.7 \%$. In contrast, Smythe et al.'s tabulated values [280] predict greater $\Delta I$ for a particle at a constant distance from the pore wall when the particle to pore size ratio increases.

As the stretch is increased the pore opening diameter increases, so that 
larger particles are able to pass through the pore. It is therefore possible that the particle size distribution broadens with stretch as larger particles become accessible. However, this explanation is inconsistent with two observations. Firstly, TRPS CVs exceed the independently measured CVs (Figure 7.6a), suggesting that broadening occurs regardless of the actual size distribution. Secondly, inclusion of larger particles does not explain the presence of two peaks in the distribution.

Figures $7.5 \mathrm{~b}, \mathrm{~d}$ and $\mathrm{f}$ provide further evidence for the correlation between off-axis transport and large pulses, showing that larger events also had longer pulse durations. Particles travelling near the pore edge in pressure-driven flow will travel more slowly than those in the centre of the flow (see Figure $7.3 \mathrm{~d}$ and elsewhere $[283,284]$ ). The same trend was observed by Tsutsui et al., [282] in that case explained by electroosmotic effects. Note that error bars in Figures 7.5b, $\mathrm{d}$ and $\mathrm{f}$ may be large when there are few events in a bin. 


\subsubsection{Pressure Variation}

The pressure applied across the membrane can be controlled using the qNano instrument's VPM. In the usual TRPS measurement protocols, pressure is varied in order to measure concentration [42], to study the influence of different transport mechanisms during charge measurements [46, $47,111]$, and to ensure stable pulse detection during size measurements. Figure 7.7 shows the measured pulse size distributions for particle set B on pores 1 and 2 at different applied pressures. As with increasing stretch, the apparent particle size distribution broadens with increasing pressure (Figures 7.7 and 7.8). Figure 7.8a shows that the $\mathrm{CV}$ for two different data sets increased well in excess of the value obtained using SEM. When the particle size distributions were fitted with two peaks, the distance between the peaks also increased with pressure (Figure 7.8b).

As Figure 7.7a inset shows, the mean pulse height decreased with increasing pressure. This observation is likely to be caused by under-sampling of the peaks, because the experiments at relatively high applied pressures could have FWHM durations shorter than $0.1 \mathrm{~ms}$ (the usual standard for other data). Under-sampling is also consistent with broadening of the size 


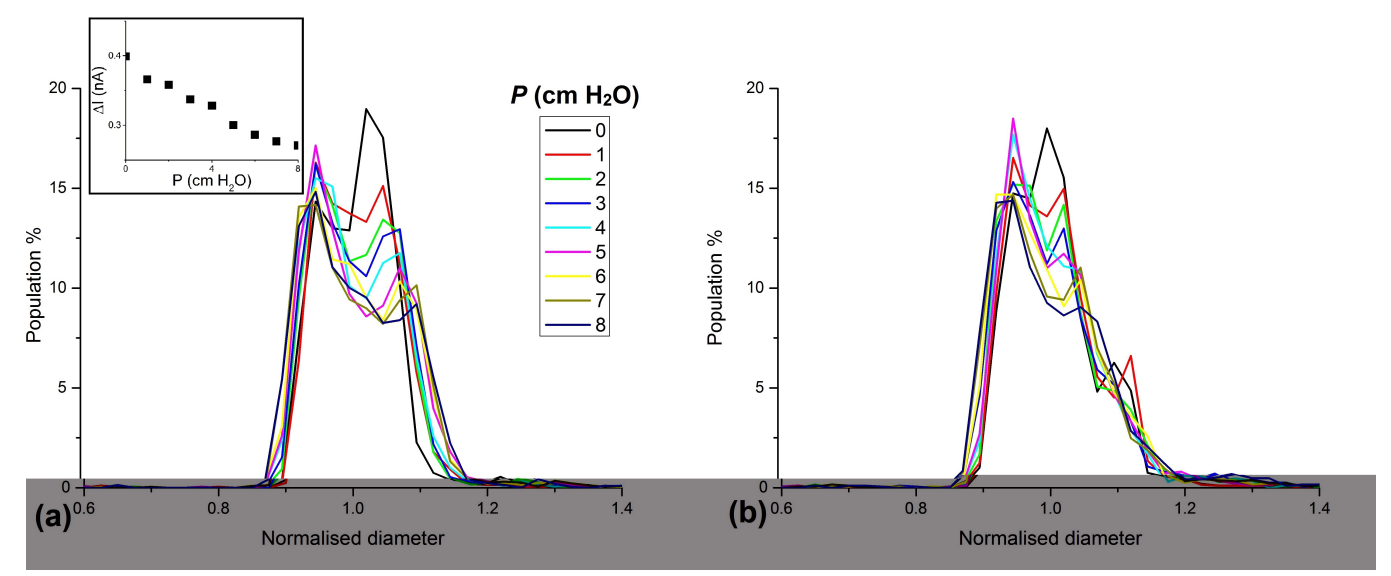

Figure 7.7: TRPS pulse size distributions as a function of applied pressure for particle set $B$ (a) on pore 1 and (b) on pore 2 are normalised by the mean blockade magnitude of the $>1000$ pulses at each pressure (inset), with bin size 0.025 .

distribution. However, this does not explain the appearance of two peaks in the data, which is again attributable to particle trajectory variation.

In Figure 7.7, it is apparent that the peak at larger values of $\Delta I$ becomes smaller at increased $P$ (relative to the other peak, Figure 7.8c). This trend was not observed for changing pore size (inset), and is not explained by under-sampling. Large $\Delta I$ pulses are produced by particles near the pore edge which travel slowly, and are therefore least affected by undersampling. It follows that those particles at the edge of the pore opening become less frequent as the pressure is increased. For simple pressuredriven pipe flow, the total volumetric flow rate $\left(\pi r^{4} P^{\prime} / 8\right.$ for radius $\left.r\right)$ is 

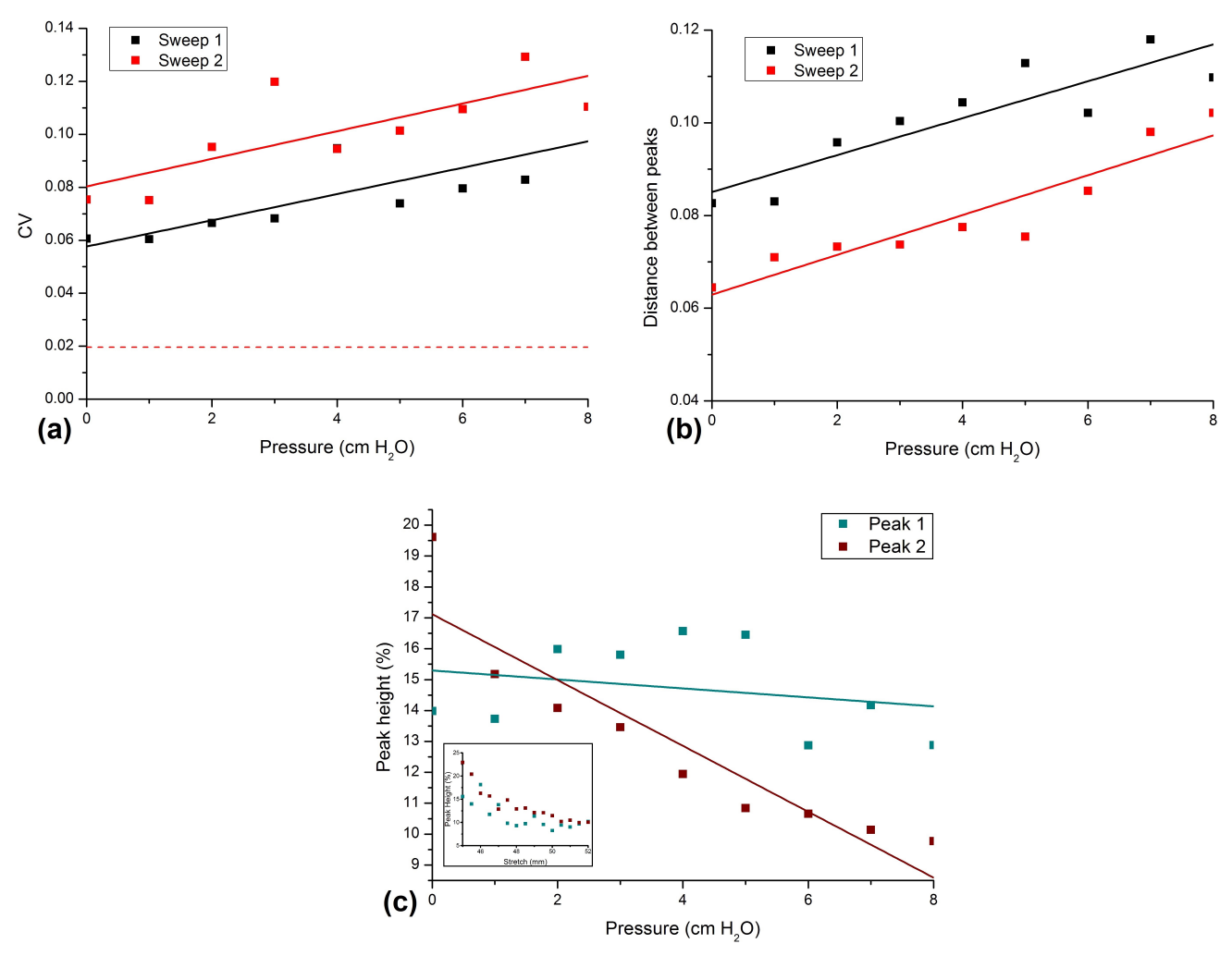

Figure 7.8: (a) Size distribution CVs for Figure 7.7a (Sweep 1) and 7.7b (Sweep 2). Dashed line indicates the CV from SEM for particle set B. (b) Separation of the positions of peaks from Figure 7.7a and $b$ (normalised diameter units) when two peaks are fitted to the distribution at each stretch. (c) Relative sizes of the peaks from Sweep 1. The brown data set occurs at higher values of $\Delta I$ than the green data set. Inset, heights of the two peaks as a function of stretch. Peak 1 occurs at lower values of $\Delta I$. Solid lines in Figures are linear fits to each data set. 
linear with respect to the pressure gradient $P^{\prime}$. Therefore the observed trend is not explained by pipe flow, because the proportion of fluid passing through an annulus at any particular distance from the centre of the pore is independent of $P^{\prime}$.

Hydrodynamic focussing means that particle trajectories may be slightly shifted towards the central axis within the pore constriction at higher pressures as seen in FEM studies (Figure 7.3), consistent with the experimental result (Figure 7.8c) that the relative size of the peak at higher $\Delta I$ decreased with increasing $P$. Hydrodynamic or inertial focussing is a non-trivial phenomenon [286, 287], and has a minor effect in the experiments presented. The low channel Reynolds number $(\operatorname{Re} \approx 0.10$ for simulations, even smaller for experimental pores) is an indicator of the degree of this effect. In comparison, Berge et al.'s observations [283] of hydrodynamically focussed particles passing through cylindrical pores have associated Reynolds numbers between 0.7 and 2.4 .

Further data (Figure 7.9) independently verify the dependence of resistive pulse distributions on pressure and stretch, including over repeated measurements (runs). These data were obtained using a different pore, particle set, and electrolyte to those used in Figs. 7.5 and 7.7. For these 


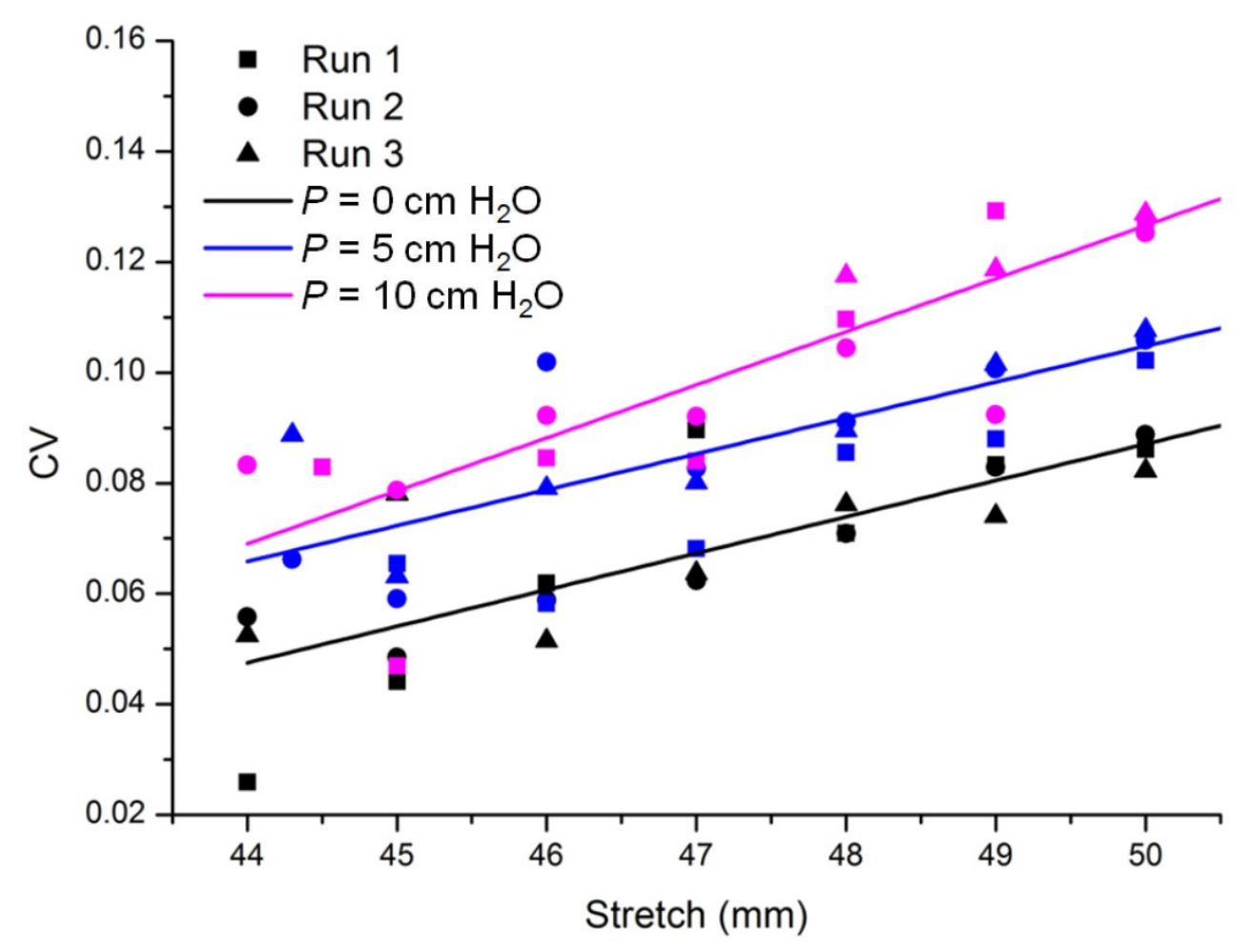

Figure 7.9: Data obtained from TRPS experiments using pore 4 and particle set $\mathrm{M}$, with $0.28 \mathrm{~V}<V_{0}<1.02 \mathrm{~V}$. CV data were extracted from pulse size distributions as in previous figures. Lines are linear fits to all data from the three runs at each pressure setting. 
experiments, $V_{0}$ was varied with stretch so that $I_{0}$ and $\Delta I$ were approximately constant between different stretch settings - in the previous experiments, $V_{0}$ was held constant and current measurements were varied with stretch. Both stretch and pressure have positive correlations with respect to distribution breadth.

\subsubsection{Pore Orientation and Applied Potential}
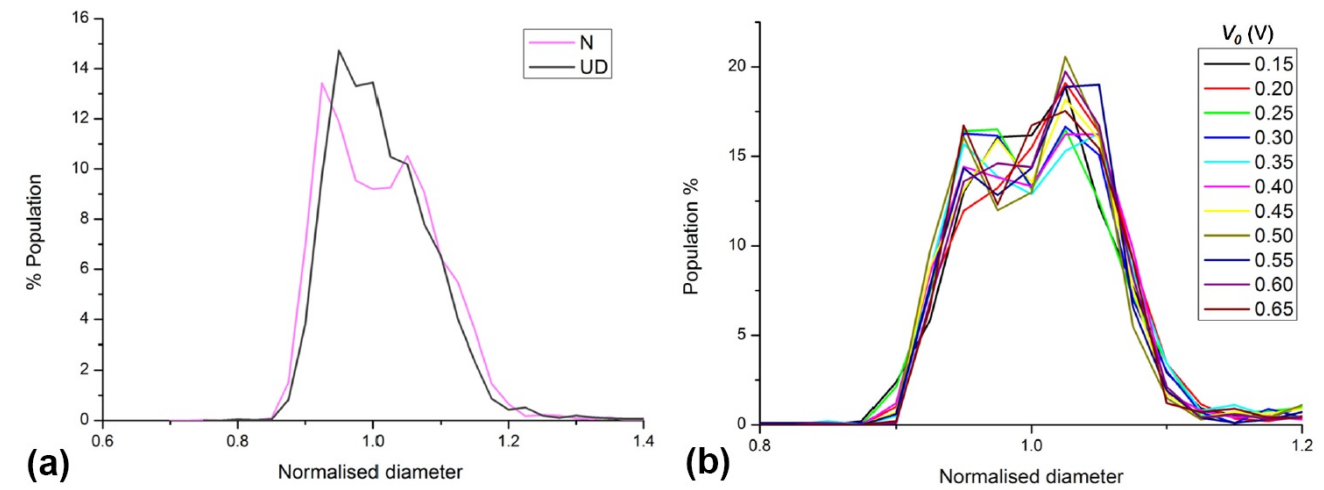

Figure 7.10: (a) Normalised TRPS resistive pulse distributions using particle set $B$ with pore 1 in the normal (N) and upside-down (UD) pore orientations, and bin size 0.025 . Data represent at least 1000 resistive pulses at $52 \mathrm{~mm}$ stretch, normalised by the mean blockade magnitude. (b) Similar data at varied applied voltage using pore 1 and particle set $B$.

Because tunable pores are conical, they can be assembled in either a normal orientation with the small opening at the top, or an upside down orientation with the large opening at the top. All experiments in this Chap- 
ter other than those presented in this Section used the normal orientation, with particles in the upper fluid cell moving downwards. When using the upside down orientation for one series of measurements, it was found that the second peak was less prominent (Figure 7.10a) and the CV in fact decreased with stretch, although this was not observed when using another pore. The experiment in Figure 7.10a suggests that the particle trajectories are less widely distributed across the width of the pore when particles move from the larger to the smaller pore opening. It is probable that hydrodynamic focussing is more effective in the upside-down configuration, as the particle must pass through the membrane within the cone before reaching the point at which the resistive pulse is generated. While in the cone, the wall force and shear lift elements of hydrodynamic focussing [287] are more significant than outside the cone. Strong focussing (possibly to an off-axis position) would result in merging of the two peaks, as observed in Figure 7.10a. Because the exact geometry is pore-dependent, this explanation also accounts for the inconsistency in data obtained from different pores.

Figure $7.10 \mathrm{~b}$ shows that the applied potential does not have an effect on the pulse size distribution, confirming that the physical origin of the 
distribution broadening is not electrokinetic in nature.

\subsubsection{Electrokinetic Effects}

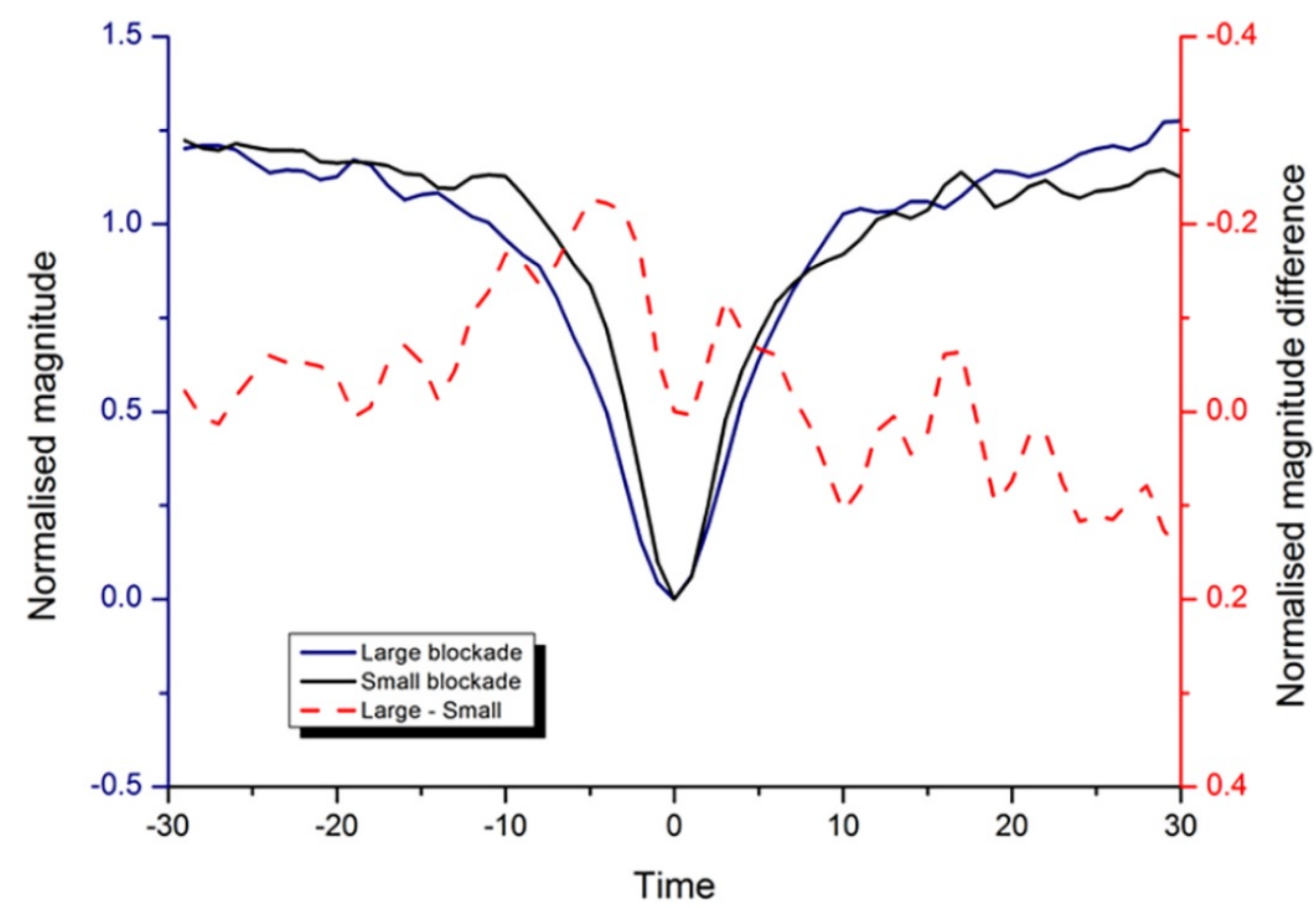

Figure 7.11: Plots of the average of 5 small and large pulses obtained using particle set B and pore 1 at $52 \mathrm{~mm}$ stretch (Figure 7.5a). The averaged pulses are normalised so that their magnitudes are equal, and the difference between pulses is plotted in red.

In Tsutsui et al.'s recent work [282], electrokinetic transport was dominant, and temporal fluctuations in particle position were simulated based on competition between electrokinetic focussing and Brownian motion (al- 
though full trajectories were not calculated). Electrokinetic focussing was provided by the electrostatic force between the membrane surface and a similarly charged particle, acting towards the centre of the pore. A key piece of experimental evidence for the importance of this focussing was that resistive pulse profiles varied prior to the pulse peak, but were mostly uniform thereafter. This suggested that the particle trajectories varied up until they entered the pore, at which point they became focussed on to the central axis.

Similar evidence for electrokinetic focussing has been observed here, in that the experimental pulse profiles suggest some differences prior to the pulse peak, and there is no evidence for large differences following the pulse peak, as predicted by simulations based on hydrodynamics alone (Figure 7.11). Electrokinetic focussing is also consistent with experimentally observed trends. With increasing stretch or higher pressure, the electrokinetic transport becomes relatively weak, so distribution broadening would be expected. In the upside-down configuration, particles can be focussed on-axis prior to generation of the pulse peak, producing a more uniform pulse size distribution. Other forms of electrokinetic transport have thus far been neglected, and this is primarily justified by the inde- 
pendence of the size distribution and the applied voltage (Figure 7.10b). The assumption is that pressure-driven flow dominates transport due to electrophoresis, electroosmosis, or DC dielectrophoresis.

It is worth discussing some expected effects if electrokinetics (other than the focussing effect) were to be significant. In contrast to pressuredriven flow and electrophoresis, electroosmosis produces a characteristic plug-like flow profile. This could change the relative flow rates between the centre and the edge of the pore, and therefore the relative size of peaks in a two-peak pulse size distribution. Another point is that the electrolyte resistivity is homogeneous in the simulations, justified by the electrical double layer thickness $(\sim 1 \mathrm{~mm})$ being much smaller than the pore and the particle sizes. However, ionic concentration polarization beyond the double layer can give rise to artefacts in RPS (Chapter 6). Although conductive and biphasic pulses are not typically important at electrolyte concentrations greater than $\sim 50 \mathrm{mM}$ in TRPS (Chapter 6), it is possible that size distributions are affected by electrolyte concentration distribution across the pore opening. However, these effects did not produce any clear experimental size distribution variations as a function of voltage. 


\subsubsection{Discussion}

The important practical consequence of the current work is reduction in uncertainty for particle size measurements made using resistive pulse techniques, in particular TRPS. It is clear that distribution broadening could exaggerate the apparent range of particle sizes. To reduce broadening, it is best to work with relatively small pore sizes, or else to limit analyses to those data within the peak at lower $\Delta I$. Real particle distributions have some dispersity, so the measured resistive pulse distribution can be interpreted as the convolution of the particle size distribution with a broadening distribution, such as those determined for single particles using pressure reversal in Figure 7.4. This process could be reversed via a deconvolution, thereby adjusting a pulse size distribution to obtain a distribution more closely reflecting the particle sizes. A similar empirical approach was used previously, reducing the measured size $\mathrm{CV}$ of a particle set from $7.1 \%$ to $3.5 \%$ [284].

Further, this study highlights the care that must be taken when interpreting experimental size distributions that have some breadth. All things considered, a distribution mode calculated using a well-defined histogram 
bin size is often the best single indicator of size, especially for monodisperse particles. Other central statistics (mean, median) are affected by outlying aggregates, distribution asymmetry [14], and thresholding [177], regardless of any broadening due to a measurement technique.

\subsection{Conclusion}

The size of a resistive pulse depends on the trajectory of a particle as it moves through the sensing pore. FEM demonstrates this effect as it applies to TRPS, and explains the appearance of two peaks in size distributions of mono-modal particles. In all experiments, pulse size distributions were broader than expected from SEM results when using the conventional volumetric sizing method. The two peaks observed move apart as the pore size increases, providing evidence that particle trajectories cause broadening of resistive pulse distributions. The reasons for distribution broadening at increasing pressures are less clear-cut, both because of possible under-sampling, and because the relative height of the peak at greater pulse size decreases with pressure. The latter observation is consistent with hydrodynamic focussing of particles towards the pore 
axis as they enter the smaller pore entrance from the adjacent half-space, as also suggested by the simulation which accounted for inertial effects. Electrokinetic effects, although present, do not seem to affect pulse sizes in the experiments, as there was no variation with $V_{0}$.

Understanding the genesis of pulse size distributions helps to define, and thereby reduce, the uncertainty in size measurements using resistive pulse sensors. In particular, distribution broadening defines an important random uncertainty for particle-by-particle analysis. The same considerations also apply to resistive pulse measurements of particle charge, shape, or for reliable discrimination between particle types. 


\section{Chapter 8}

\section{Conclusions and Future Work}

This Thesis has rigorously studied TRPS through a thorough investigation of particle and pore surface charge measurements and in-depth examination of biphasic pulses and particle size distribution broadening. To the existing body of work this Thesis adds an exhaustive review of applications of TRPS, and adds to the existing knowledge of the physical phenomena present in TRPS pores. TRPS research covers a wide range of particle types, encompassing numerous research fields and research objectives. Advancement of this technology requires a better understanding of the technique, particularly in the area of particle surface charge, and the 
research presented in this Thesis helps to provide that understanding. In this Chapter important results and advances are summarised and possible future work is discussed.

\section{1 $\zeta$-potential Measurements}

In Chapter 4 detailed theory and background of $\zeta$-potential and its measurement using TRPS were presented and discussed. A number of $\zeta$ potential measurement methods were outlined including a number of multipoint analysis methods in which the blockade was split into fractions and the electrokinetic component of each fraction was derived. The uncertainties associated with each method were described.

Method I and Method II were compared for charged and uncharged particles and it was found that Method II was superior discerning the particle types, regardless of pore geometry. Detailed error analysis of Method II found that blockade fractions closer to $n=0.3$ and measurements at higher voltages resulted in lower uncertainties. The large uncertainty in absolute values of $\zeta_{\text {particle }}$ for this method was due to Equation 4.11 and the combining of errors using Equation 4.12. This analysis was done without 
the errors associated with $\zeta_{C a l}$ and $\zeta_{\text {pore }}$.

Calibration $\zeta$-potential values were then obtained with DLS using two different DLS measurement cells, one of which was discovered to be of superior quality. This allowed for the measurement of $\zeta_{C a l}$ for a range of Izon Science calibration particles including those to be used in later measurements.

$\zeta$-potential measurements of nominally $200 \mathrm{~nm}$ particles each with a nominal surface charge were achieved in 3 different electrolytes using Method III, and in a single electrolyte at 11 concentrations using Method IV. For both DLS and TRPS there was no clear trend or difference observed between electrolytes. An increase in $\zeta_{\text {particle }}$ was seen with increasing electrolyte concentration as expected due to EDL compression at higher electrolyte concentrations. Trends with nominal particle surface charge were clearer with DLS than TRPS.

Detailed error analysis of Method IV was achieved, incorporating measured $\zeta_{C a l}$ and its related uncertainty. It was found that measurements at $n=0.2-0.45$ resulted in lower uncertainties. Lower uncertainties than Method II were observed due to less sources of random error. This anal- 
ysis was done without errors associated with $\zeta_{\text {pore }}$ which would further improve this method. The large uncertainty in absolute values of $\zeta_{\text {particle }}$ for this method is due to incorporating $\zeta_{\mathrm{Cal}}$ and the combining of errors using Equation 4.12.

An initial investigation of $\mathrm{pH}$ variation was attempted. However, difficulties with surfactant effects on $\zeta$-potential trends were encountered using DLS. TRPS measurements of particles without surfactant were unsuccessful at all attempted $\mathrm{pHs}$ due to pore blocking. It was suggested that the issue of surfactant affecting $\zeta$-potential measurements and pore blocking (which is a commonly occurring problem in TRPS) could be addressed concurrently by applying non-fouling pore coatings.

In Chapter 5, $\zeta_{\text {particle }}$ measurements using Method IV were continued with the addition of streaming potential measurements so that $\zeta_{\text {pore }}$ could be obtained using the qNano. $\zeta_{\text {pore }}$ measurements were made at a range of different pore sizes and then combined with $\zeta_{\text {particle }}$ measurements to show that charge measurements could be made at a range of stretches. $\zeta_{\text {pore }}$ and $\zeta_{\text {particle }}$ measurements were made at a range of different electrolyte concentrations and $\mathrm{pHs}$. The isoelectric point of TPU was obtained. Random uncertainties using Method IV have been shown to be less than 
previously investigated methods and the inclusion of measured calibration $\zeta_{\text {particle }}$ and $\zeta_{\text {pore }}$ values reduces the systematic uncertainty.

In Chapter 5, although uncertainties were better characterized and understood than Chapter 4 , trends with respect to $\mathrm{pH}$, electrolyte concentration and manufacturers specifications for charge were unclear, as they were for DLS measurements. These represent some of the ongoing issues for $\zeta$-potential measurements along with measurement and control of pore surface charge.

Chapters 4 and 5 represent a thorough investigation into charge measurements using TRPS. For the most part, previous investigations of $\zeta_{\text {particle }}$ measurement using TRPS have only compared a few particle types in one set of conditions $[14,112,114]$. These previous studies also use a single pre-assigned value of $\zeta_{\text {pore }}$ even when working in biological conditions which can affect pore surface properties. The work presented in these two Chapters builds on other detailed investigations of charge measurement with TRPS [47, 111], by examining a large number of particle sets in a range conditions, as well as addressing the issue of measuring $\zeta_{\text {pore }}$.

In addition to particle charge measurements, in Chapter 5, a number 
of types of coatings were successfully applied to TPU pores. Pore coatings were analysed using XPS, FTIR, $I-V$ curves and streaming potential measurements. Pore coatings have not been previously investigated using TRPS so are an interesting expansion of the current technology. However, in this research coating removed the ability of a pore to measure particles. Streaming potential measurements of coated pores in general agreed with ICR in terms of whether the pore was negatively or positively charged. Often ICR was a more consistent indicator of surface charge especially for dopamine coated pores where the ICR indicated a more negatively charged pore but $\zeta_{\text {pore }}$ indicated a more positive one. This is most likely because of uneven pore coating of the complicated pore geometry. Large current oscillations were observed with coated pores, possibly due to nanoprecipitation. Improvement of pore coatings to allow for particle measurement and complete coating of the pore (so that ICR agrees with $\left.\zeta_{\text {pore }}\right)$, as well as further investigation of the large current oscillations are all areas for future research. 


\subsection{Biphasic Pulses}

In Chapter 6, TRPS was used to study biphasic pulses, in particular their conductive component, as a function of several quantified experimental variables. The chronological ordering of each biphasic pulse was consistent with concentration polarization, where the conductive component was generated as the particle passes through the ion depletion region. Results obtained at varied applied pressure indicated that the ionic depletion region could be shifted in the direction of flow. A quantitative link between conductive pulse magnitude and excess charge in the EDL was suggested by the linear relation between conductive pulse magnitude and particle surface group density. Conductive pulse magnitude was seen to monotonically decrease with increasing electrolyte concentration, and the onset concentration was $\sim 50 \mathrm{mM}$. The ratio of conductive to resistive pulse magnitudes decreased with increases in both negative potential and pore size, indicating a difference in mechanisms between conductive and resistive pulses. This work will be important for TRPS users, especially if operating below conventional (or physiological) salt concentrations of $\sim 100 \mathrm{mM}$. Many of the observed trends will extend to other RPS techniques operating at similar length scales, although specific ma- 
terials and pore geometries affect quantitative results. The size-tunable function of TRPS suggests further experiments which could contribute to the fundamental understanding of conductive and biphasic pulses. Also, the demonstrated relationship between biphasic pulses and particle surface charge suggests a possible method for surface charge measurement, as well as applications in particle discrimination and monitoring of onbead chemistry.

\subsection{Distribution Broadening}

Chapter 7 investigated the breadth of pulse size distributions using nominally monodisperse particle size standards. The size of a resistive pulse depends on the trajectory of a particle as it moves through the sensing pore. FEM demonstrated this effect as it applies to TRPS, and explained the appearance of two peaks in size distributions of mono-modal particles. In all experiments, pulse size distributions were broader than expected from SEM results when using the conventional volumetric sizing method. The two peaks observed moved apart as the pore size increased, providing evidence that particle trajectories cause broadening of resistive pulse dis- 
tributions. The reasons for distribution broadening at increasing pressures were less clear-cut, both because of possible under-sampling, and because the relative height of the peak at greater pulse size decreased with pressure. The latter observation was consistent with hydrodynamic focussing of particles towards the pore axis as they enter the smaller pore entrance from the adjacent half-space, as also suggested by the simulation which accounted for inertial effects. Electrokinetic effects, although present, did not seem to affect pulse sizes in the experiments, as there was no variation with $V_{0}$.

Understanding the genesis of pulse size distributions helps to define, and thereby reduce, the uncertainty in size measurements using resistive pulse sensors. In particular, distribution broadening defines an important random uncertainty for particle-by-particle analysis. The same considerations also apply to resistive pulse measurements of particle charge, shape, or for reliable discrimination between particle types. 


\subsection{Future work}

A thorough investigation into $\zeta$-potential measurements was undertaken here. However, there is still a lack of consistency between TRPS, DLS and expected values from the manufacturer. Potential ways to address this include using pore coatings to remove the need for surfactant and control the pore surface charge, having more control over particle synthesis, or accounting for particle trajectories in $\zeta$-potential measurements. More $\zeta$-potential measurements for a wider range of particles would be beneficial including biological particles such as EVs where much of the recent TRPS research focuses. Biphasic pulses could also be applied to these discrimination experiments as conductive pulses have been shown to be an indicator of surface charge density. A number of coating methods were applied to TPU pores. However, particles were never able to measured on these coated pores. An investigation into coating pores, in particular anti-fouling coatings, so that they could still be used for conventional TRPS would improve the technique. The oscillations observed with coated pores were only investigated briefly here but a more intensive look at this phenomenon could give further information about coating stability and more generally ICR. 


\section{Bibliography}

[1] Doane, T. L.; Burda, C. Chem. Soc. Rev. 2012, 41, 2885-2911.

[2] Salata, O. V. J. Nanobiotechnology 2004, 2, DOI: $10.1186 / 1477-$ $3155-2-3$

[3] Zhu, Y.; Liao, L. J. Nanosci. Nanotechnol. 2015, 15, 4753-4773.

[4] Boisseau, P.; Lahmani, M., Nanoscience: Nanobiotechnology and Nanobiology; Springer Science \& Business Media, Heidelberg: 2009.

[5] Kruis, F. E.; Fissan, H.; Peled, A. J. Aerosol Sci. 1998, 29, 511-535.

[6] Chen, X.; Mao, S. S. Chem. Rev. 2007, 107, 2891-2959.

[7] Sozer, N.; Kokini, J. L. Trends Biotechnol. 2009, 27, 82-89.

[8] Spencer, D. S.; Puranik, A. S.; Peppas, N. A. Curr. Opin. Chem. Eng. $2015,7,84-92$. 
[9] Zeng, S.; Yong, K.-T.; Roy, I.; Dinh, X.-Q.; Yu, X.; Luan, F. Plasmonics 2011, 6, 491-506.

[10] Zetasizer Nano Series User Manual., http : / / www . malvern . com/en/support/resource-center/user-manuals/MAN0 485EN. aspx, 2013 (accessed October 27, 2016).

[11] Berne, B. J.; Pecora, R., Dynamic light scattering: with applications to chemistry, biology, and physics; Dover Publications, New York: 2000.

[12] Anderson, W.; Kozak, D.; Coleman, V. A.; Jämting, Å. K.; Trau, M. J. Coll. Interf. Sci. 2013, 405, 322-330.

[13] Donald, A. M. Nat. Mater. 2003, 2, 511-516.

[14] Somerville, J. A.; Willmott, G. R.; Eldridge, J.; Griffiths, M.; McGrath, K. M. J. Coll. Interf. Sci. 2012, 394, 243-251.

[15] Coulter, W. H. Means for counting particles suspended in a fluid. US Patent, 2,656,508, 1953.

[16] Simson, E. Int. J. Lab Hematol. 2013, 35, 230-236.

[17] Bayley, H.; Martin, C. R. Chem. Rev. 2000, 100, 2575-2594.

[18] DeBlois, R. W.; Bean, C. P. Rev. Sci. Instrum. 1970, 41, 909-916. 
[19] DeBlois, R. W.; Bean, C. P.; Wesley, R. K. A. J. Coll. Interf. Sci. 1977, $61,323-335$.

[20] DeBlois, R. W.; Wesley, R. K. J. Virol. 1977, 23, 227-233.

[21] DeBlois, R. W.; Uzgiris, E. E.; Cluxton, D. H.; Mazzone, H. M. Anal. Biochem. 1978, 90, 273-288.

[22] Deamer, D.; Akeson, M.; Branton, D. Nat. Biotechnol. 2016, 34, 518524.

[23] Kasianowicz, J. J.; Brandin, E.; Branton, D.; Deamer, D. W. Proc. Natl. Acad. Sci. 1996, 93, 13770-13773.

[24] Vercoutere, W. A.; Winters-Hilt, S.; DeGuzman, V. S.; Deamer, D.; Ridino, S. E.; Rodgers, J. T.; Olsen, H. E.; Marziali, A.; Akeson, M. Nucleic Acids Res. 2003, 31, 1311-1318.

[25] Dekker, C. Nat. Nanotechnol. 2007, 2, 209-215.

[26] Oxford Nanopore Technologies., http : / / www . nanoporetech. com, 2008 (accessed August 10, 2016).

[27] Mikheyev, A. S.; Tin, M. M. Mol. Ecol. Resour. 2014, 14, 1097-1102.

[28] Laver, T.; Harrison, J.; O’Neill, P.; Moore, K.; Farbos, A.; Paszkiewicz, K.; Studholme, D. J. Biomol. Detect. Quantif. 2015, 3, 1-8. 
[29] Venkatesan, B. M.; Bashir, R. Nat. Nanotechnol. 2011, 6, 615-624.

[30] Branton, D.; Deamer, D. W.; Marziali, A.; Bayley, H.; Benner, S. A.; Butler, T.; Di Ventra, M.; Garaj, S.; Hibbs, A.; Huang, X. Nat. Biotechnol. 2008, 26, 1146-1153.

[31] Deamer, D. W.; Branton, D. Acc. Chem. Res. 2002, 35, 817-825.

[32] Rhee, M.; Burns, M. A. Trends Biotechnol. 2007, 25, 174-181.

[33] Sexton, L. T.; Horne, L. P.; Martin, C. R. Mol. Biosyst. 2007, 3, 667685.

[34] Healy, K.; Schiedt, B.; Morrison, A. P. Future Med. 2007, 2, 875-897.

[35] Kasianowicz, J. J.; Robertson, J. W.; Chan, E. R.; Reiner, J. E.; Stanford, V. M. Annu. Rev. Anal. Chem. 2008, 1, 737-766.

[36] Healy, K. Future Med. 2007, 2, 459-481.

[37] Gu, L.-Q.; Shim, J. W. Analyst 2010, 135, 441-451.

[38] Haque, F.; Li, J.; Wu, H.-C.; Liang, X.-J.; Guo, P. Nano Today 2013, 8, $56-74$

[39] Howorka, S.; Siwy, Z. Chem. Soc. Rev. 2009, 38, 2360-2384.

[40] Kocer, A.; Tauk, L.; Dejardin, P. Biosens. Bioelectron. 2012, 38, 1-10. 
[41] Sowerby, S. J.; Broom, M. F.; Petersen, G. B. Sensor Actuat. B Chem. 2007, 123, 325-330.

[42] Willmott, G. R.; Vogel, R.; Yu, S. S. C.; Groenewegen, L. G.; Roberts, G. S.; Kozak, D.; Anderson, W.; Trau, M. J. Phys. Condens. Matter 2010, 22, 454116 .

[43] Vogel, R.; Willmott, G.; Kozak, D.; Roberts, G. S.; Anderson, W.; Groenewegen, L.; Glossop, B.; Barnett, A.; Turner, A.; Trau, M. Anal. Chem. 2011, 83, 3499-3506.

[44] Roberts, G. S.; Yu, S.; Zeng, Q.; Chan, L. C. L.; Anderson, W.; Colby, A. H.; Grinstaff, M. W.; Reid, S.; Vogel, R. Biosens. Bioelectron. 2012, $31,17-25$.

[45] Willmott, G. R.; Bauerfeind, L. H. Industrial Research Limited Report \#2385 2010.

[46] Kozak, D.; Anderson, W.; Vogel, R.; Chen, S.; Antaw, F.; Trau, M. ACS Nano 2012, 6, 6990-6997.

[47] Vogel, R.; Anderson, W.; Eldridge, J.; Glossop, B.; Willmott, G. Anal. Chem. 2012, 84, 3125-3131. 
[48] Weatherall, E.; Willmott, G. R.; Glossop, B. Seventh International Conference on Sensing Technology (ICST) 2013, DOI: 10 . 1109 / ICSensT . 2013.6727775 .

[49] Kozak, D.; Anderson, W.; Vogel, R.; Trau, M. Nano Today 2011, 6, 531-545.

[50] Eldridge, J.; Colby, A.; Willmott, G. R.; Yu, S.; Grinstaff, M. In Selected Topics in Nanomedicine, Chang, T. M. S., Ed.; World Scientific, Singapore; Chapter 10, pp 219-255.

[51] Willmott, G. R.; Broom, M. F.; Jansen, M. L.; Young, R. M.; Arnold, W. M. In Molecular- and Nano-Tubes, Hayden, O., Nielsch, K., Eds.; Springer, USA: 2011; Chapter 7, pp 209-261.

[52] Willmott, G. R.; Moore, P. W. Nanotechnology 2008, 19, 475-504.

[53] Roberts, G. S.; Kozak, D.; Anderson, W.; Broom, F. M.; Vogel, R.; Trau, M. Small 2010, 6, 2653-2658.

[54] Izon Science Ltd., http : / / www . izon . com, 2014 (accessed October 6, 2016).

[55] Heider, S.; Metzner, C. Virology 2014, 462, 199-206. 
[56] Arjmandi, N.; Van Roy, W.; Lagae, L.; Borghs, G. Anal. Chem. 2012, $84,8490-8496$.

[57] Park, S.; Lim, J.; Pak, Y. E.; Moon, S.; Song, Y.-K. Sensors 2013, 13, 6900-6909.

[58] Ito, T.; Sun, L.; Crooks, R. M. Anal. Chem. 2003, 75, 2399-2406.

[59] Ito, T.; Sun, L.; Bevan, M. A.; Crooks, R. M. Langmuir 2004, 20, 69406945.

[60] Saleh, O. A.; Sohn, L. L. Nano Lett. 2003, 3, 37-38.

[61] Heins, E. A.; Siwy, Z. S.; Baker, L. A.; Martin, C. R. Nano Lett. 2005, $5,1824-1829$.

[62] Wharton, J. E.; Jin, P.; Sexton, L. T.; Horne, L. P.; Sherrill, S. A.; Mino, W. K.; Martin, C. R. Small 2007, 3, 1424-1430.

[63] Uram, J. D.; Ke, K.; Mayer, M. ACS Nano 2008, 2, 857-872.

[64] Uram, J. D.; Ke, K.; Hunt, A. J.; Mayer, M. Angew. Chem. Int. Ed. $2006,45,2281-2285$.

[65] Uram, J. D.; Ke, K.; Hunt, A. J.; Mayer, M. Small 2006, 2, 967-972.

[66] An, R.; Uram, J. D.; Yusko, E. C.; Ke, K.; Mayer, M.; Hunt, A. J. Opt. Lett. 2008, 33, 1153-1155. 
[67] Steinbock, L. J.; Stober, G.; Keyser, U. F. Biosens. Bioelectron. 2009, 24, 2423-2427.

[68] Stober, G.; Steinbock, L.; Keyser, U. J. Appl. Phys. 2009, 105, 084702.

[69] German, S. R.; Luo, L.; White, H. S.; Mega, T. L. J. Phys. Chem. C 2012, 117, 703-711.

[70] Lan, W.-J.; White, H. S. ACS Nano 2012, 6, 1757-1765.

[71] Wang, G.; Zhang, B.; Wayment, J. R.; Harris, J. M.; White, H. S. J. Am. Chem. Soc. 2006, 128, 7679-7686.

[72] Lan, W.-J.; Holden, D. A.; Liu, J.; White, H. S. J. Phys. Chem. C 2011, $115,18445-18452$.

[73] Henriquez, R. R.; Ito, T.; Sun, L.; Crooks, R. M. Analyst 2004, 129, $478-482$.

[74] Luo, L.; German, S. R.; Lan, W.-J.; Holden, D. A.; Mega, T. L.; White, H. S. Annu. Rev. Anal. Chem. 2013, 7, 513-535.

[75] Wu, S.; Park, S. R.; Ling, X. S. Nano Lett. 2006, 6, 2571-2576.

[76] Lan, W.-J.; Holden, D. A.; Zhang, B.; White, H. S. Anal. Chem. 2011, $83,3840-3847$.

[77] Weatherall, E.; Willmott, G. R. Analyst 2015, 140, 3318-3334. 
[78] Willmott, G. R.; Chaturvedi, R.; Cummins, S. J. W.; Groenewegen, L. G. Exp. Mech. 2014, 54, 153-163.

[79] Willmott, G. R.; Parry, B. E. T. J. Appl. Phys. 2011, 109, 094307.

[80] Willmott, G.; Young, R. AIP Conference Proceedings 2009, 1151, 153156.

[81] Jansen, M. L.; Willmott, G. R.; Hoek, I.; Arnold, W. M. Measurement $2013,46,3560-3567$.

[82] Kozak, D.; Anderson, W.; Grevett, M.; Trau, M. J. Phys. Chem. C 2012, 116, 8554-8561.

[83] Willmott, G. R.; Platt, M.; Lee, G. U. Biomicrofluidics 2012, 6, 014103.

[84] Kozak, D.; Anderson, W.; Trau, M. Chem. Lett. 2012, 41, 1134-1136.

[85] Hauer, P.; Le Ru, E. C.; Willmott, G. R. Biomicrofluidics 2015, 9, 014110.

[86] Hauer, P. Coordinated Detection of Micro- and Nanoparticles using Tunable Resistive Pulse Sensing and Optical Spectroscopy., Ph.D. Thesis, Victoria University of Wellington, 2016.

[87] Lan, W.; Kubeil, C.; Xiong, J.; Bund, A.; White, H. S. J. Phys. Chem. C 2014, 118, 2726-2734.

[88] Willmott, G.; Smith, B. ANZIAM J. 2014, 55, 197-213. 
[89] Hall, J. E. J. Gen. Physiol. 1975, 66, 531-532.

[90] Schoch, R. B.; Han, J.; Renaud, P. Rev. Mod. Phys. 2008, 80, 839-883.

[91] Willmott, G. R.; Fisk, M. G.; Eldridge, J. Biomicrofluidics 2013, 7, 064106.

[92] Terejánszky, P.; Makra, I.; Furjes, P.; Gyurcsanyi, R. E. Anal. Chem. 2014, 86, 4688-4697.

[93] Bacri, L.; Oukhaled, A.; Schiedt, B.; Patriarche, G.; Bourhis, E.; Gierak, J.; Pelta, J.; Auvray, L. J. Phys. Chem. B 2011, 115, 2890-2898.

[94] Willmott, G. R.; Smith, B. G. Nanotechnology 2012, 23, 088001.

[95] Lee, S.; Zhang, Y.; White, H. S.; Harrell, C. C.; Martin, C. R. Anal. Chem. 2004, 76, 6108-6115.

[96] Allen, C. S.; Loo, J. F. C.; Yu, S.; Kong, S. K.; Chan, T.-F. Appl. Microbiol. Biotechnol. 2014, 98, 855-862.

[97] Yang, L.; Broom, M. F.; Tucker, I. G. Pharm. Res. 2012, 29, 2578-2586.

[98] De Vrij, J.; Maas, S. L. N.; van Nispen, M.; Sena-Esteves, M.; Limpens, R. W. A.; Koster, A. J.; Leenstra, S.; Lamfers, M. L.; Broekman, M. L. D. Nanomedicine 2013, 1443-1458. 
[99] Eldridge, J. Nanoparticle Charge and Shape Measurements using Tuneable Resistive Pulse Sensing., Ph.D. Thesis, Victoria University of Wellington, 2016.

[100] Maxwell, J. C., A Treatise on Electricity and Magnetism; Clarendon press, Oxford: 1881; Vol. 1.

[101] Rayleigh, L. Philos. Mag. 1892, 34, 481-502.

[102] Van der Pol, E.; Hoekstra, A.; Sturk, A.; Otto, C.; Van Leeuwen, T.; Nieuwland, R. J. Thromb. Haemost. 2010, 8, 2596-2607.

[103] Van der Pol, E.; Coumans, F.; Varga, Z.; Krumrey, M.; Nieuwland, R. J. Thromb. Haemost. 2013, 11, 36-45.

[104] Arjmandi, N.; Van Roy, W.; Lagae, L. Anal. Chem. 2014, 86, 46374641.

[105] McDaniel, J. R.; Weitzhandler, I.; Prevost, S.; Vargo, K. B.; Appavou, M.-S.; Hammer, D. A.; Gradzielski, M.; Chilkoti, A. Nano Lett. 2014, $14,6590-6598$.

[106] Platt, M.; Willmott, G. R.; Lee, G. U. Small 2012, 8, 2436-2444.

[107] Billinge, E. R.; Muzard, J.; Platt, M. J. Nanosci. Nanotechnol. 2013, 1, DOI: $10.7243 / 2053-0927-1-1$. 
[108] Ang, Y. S.; Yung, L.-Y. L. ACS Nano 2012, 6, 8815-8823.

[109] Ito, T.; Sun, L.; Henriquez, R. R.; Crooks, R. M. Acc. Chem. Res. 2004, $37,937-945$.

[110] Harms, Z. D.; Mogensen, K. B.; Nunes, P. S.; Zhou, K.; Hildenbrand, B. W.; Mitra, I.; Tan, Z.; Zlotnick, A.; Kutter, J. P.; Jacobson, S. C. Anal. Chem. 2011, 83, 9573-9578.

[111] Eldridge, J. A.; Willmott, G. R.; and, W. A.; Vogel, R. J. Coll. Interf. Sci. 2014, 429, 45-52.

[112] Blundell, E. L.; Healey, M. J.; Holton, E.; Sivakumaran, M.; Manstana, S.; Platt, M. Anal. Bioanal. Chem. 2016, 408, 5757-5768.

[113] Blundell, E. L. C. J.; Mayne, L.; Lickorish, M.; Christie, S.; Platt, M. Farad. Discuss. 2016, Advance Article, DOI: 10.1039 /C6FD 00072 J.

[114] Sikora, A.; Shard, A. G.; Minelli, C. Langmuir 2016, 32, 2216-2224.

[115] Kozak, D.; Broom, M.; Vogel, R. Curr. Drug Deliv. 2014, 12, 115-120.

[116] Maas, S. L. N.; de Vrij, J.; van der Vlist, E. J.; Geragousian, B.; van Bloois, L.; Mastrobattista, E.; Schiffelers, R.; Wauben, M. H. M.; Broekman, M. L.; Nolte, E. N. M. J. Control. Release 2014, 200, 87-96. 
[117] Booth, M. A.; Vogel, R.; Curran, J. M.; Harbison, S.; Travas-Sejdic, J. Biosens. Bioelectron. 2013, 45, 136-140.

[118] Low, M.; Yu, S.; Han, M. Y.; Su, X. Aust. J. Chem. 2011, 64, 1229-1234.

[119] Alsager, O. A.; Kumar, S.; Willmott, G. R.; McNatty, K. P.; Hodgkiss, J. M. Biosens. Bioelectron. 2014, 57, 262-268.

[120] Chiang, Y.-T.; Cheng, Y.-T.; Lu, C.-Y.; Yen, Y.-W.; Yu, L.-Y.; Yu, K.-S.; Lyu, S.-Y.; Yang, C.-Y.; Lo, C.-L. Chem. Mater. 2013, 25, 4364-4372.

[121] Chung, M.-C.; Dean, S.; Marakasova, E. S.; Nwabueze, A. O.; van Hoek, M. L. PloS one 2014, 9, e93119.

[122] Rabanel, J.-M.; Hildgen, P.; Banquy, X. J. Control. Release 2014, 185, 71-87.

[123] Brar, S. K.; Verma, M. Trends Anal. Chem. 2011, 30, 4-17.

[124] Bell, N. C.; Minelli, C.; Tompkins, J.; Stevens, M. M.; Shard, A. G. Langmuir 2012, 28, 10860-10872.

[125] Varga, Z.; Yuana, Y.; Grootemaat, A. E.; Van der Pol, E.; Gollwitzer, C.; Krumrey, M.; Nieuwland, R. J. Extracell. Vesicles 2014, 3, 23298.

[126] Varenne, F.; Makky, A.; Gaucher-Delmas, M.; Violleau, F.; Vauthier, C. Pharm. Res. 2016, 33, 1220-1234. 
[127] Domingos, R. F.; Baalousha, M. A.; Ju-Nam, Y.; Reid, M. M.; Tufenkji, N.; Lead, J. R.; Leppard, G. G.; Wilkinson, K. J. Environ. Sci. Technol. 2009, 43, 7277-7284.

[128] Sivakumaran, M.; Platt, M. Nanomedicine 2016, 11, 2197-2214.

[129] Salih, M.; Zietse, R.; Hoorn, E. J. Am. J. Physiol. Renal Physiol. 2014, $306,1251-1259$.

[130] Kathe, N.; Henriksen, B.; Chauhan, H. Drug Dev. Ind. Pharm. 2014, 40, 1565-1575.

[131] Pal, A. K.; Aalaei, I.; Gadde, S.; Gaines, P.; Schmidt, D.; Demokritou, P.; Bello, D. ACS Nano 2014, 8, 9003-9015.

[132] Böing, A.; Stap, J.; Hau, C.; Afink, G.; Ris-Stalpers, C.; Reits, E.; Sturk, A.; van Noorden, C.; Nieuwland, R. Biochim. Biophys. Acta Mol. Cell Res. 2013, 1833, 1844-1852.

[133] Burnouf, T.; Goubran, H. A.; Chen, T.-M.; Ou, K.-L.; El-Ekiaby, M.; Radosevic, M. Blood Rev. 2013, 27, 77-89.

[134] Patko, D.; Gyorgy, B.; Nemeth, A.; Szabó-Taylor, K.; Kittel, A.; Buzás, E. I.; Horvath, R. Sensor Actuat. B Chem. 2013, 188, 697-701. 
[135] Lane, R. E.; Korbie, D.; Anderson, W.; Vaidyanathan, R.; Trau, M. Sci. Rep. 2015, 5, 7639.

[136] Farkas, K.; Pang, L.; Lin, S.; Williamson, W.; Easingwood, R.; Fredericks, R.; Jaffer, M. A.; Varsani, A. Food Environ. Virol. 2013, 5, 231235.

[137] Coumans, F. A.; van der Pol, E.; Böing, A. N.; Hajji, N.; Sturk, G.; van Leeuwen, T. G.; Nieuwland, R. J. Extracell. Vesicles 2014, 3, DOI: $10.3402 / \mathrm{jev} \cdot \mathrm{v} 3.25922$.

[138] Van Bracht, E.; Stolle, S.; Hafmans, T. G.; Boerman, O. C.; Oosterwijk, E.; van Kuppevelt, T. H.; Daamen, W. F. Eur. J. Pharm. Biopharm. 2014, 87, 80-89.

[139] Yang, A. K. L.; Lu, H.; Wu, S. Y.; Kwok, H. C.; Ho, H. P.; Yu, S.; Cheung, A. K. L.; Kong, S. K. Anal. Chim. Acta 2013, 782, 46-53.

[140] Cheung, A. K. L.; Yang, A. K. L.; Ngai, B. H.; Samuel, S. C.; Gao, M.; Lau, P. M.; Kong, S. K. Analyst 2015, 140, 1337-1348.

[141] Parmentier, J.; Thomas, N.; Müllertz, A.; Fricker, G.; Rades, T. Int. J. Pharm. 2012, 437, 253-263.

[142] Gazzola, D.; Van Sluyter, S. C.; Curioni, A.; Waters, E. J.; Marangon, M. J. Agric. Food Chem. 2012, 60, 10666-10673. 
[143] Kühnemund, M.; Nilsson, M. Biosens. Bioelectron. 2015, 67, 11-17.

[144] Witzigmann, D.; Wu, D.; Schenk, S. H.; Balasubramanian, V.; Meier, W.; Huwyler, J. ACS Appl. Mater. Interfaces 2015, 7, 10446-10456.

[145] Billinge, E. R.; Broom, M.; Platt, M. Anal. Chem. 2013, 86, 1030-1037.

[146] Billinge, E. R.; Platt, M. Biosens. Bioelectron. 2015, 68, 741-748.

[147] Lai, K. K.; Renneberg, R.; Mak, W. C. RSC Adv. 2014, 4, 11802-11810.

[148] Pan, B.-T.; Johnstone, R. M. Cell 1983, 33, 967-978.

[149] Raposo, G.; Nijman, H. W.; Stoorvogel, W.; Liejendekker, R.; Harding, C. V.; Melief, C. J.; Geuze, H. J. J. Exp. Med. 1996, 183, 11611172.

[150] Andaloussi, S. E.; Mäger, I.; Breakefield, X. O.; Wood, M. J. Nat. Rev. Drug Discov. 2013, 12, 347-357.

[151] Raposo, G.; Stoorvogel, W. J. Cell Biol. 2013, 200, 373-383.

[152] Meel, R.; Krawczyk-Durka, M.; Solinge, W.; Schiffelers, R. Int. J. Lab Hematol. 2014, 36, 244-253.

[153] Momen-Heravi, F.; Balaj, L.; Alian, S.; Tigges, J.; Toxavidis, V.; Ericsson, M.; Distel, R. J.; Ivanov, A. R.; Skog, J.; Kuo, W. P. Front. Physiol. $2012,3,354$. 
[154] Witwer, K. W.; Buzas, E. I.; Bemis, L. T.; Bora, A.; Lässer, C.; Lötvall, J.; Nolte, E. N., et al. J. Extracell. Vesicles 2013, 2, 20360.

[155] Hexley, P.; Rismiller, K. P.; Robinson, C. T.; Babcock, G. F. Exosomes Microvesicles 2014, 2, 1-7.

[156] Akers, J. C.; Ramakrishnan, V.; Nolan, J. P.; Duggan, E.; Fu, C.-C.; Hochberg, F. H.; Chen, C. C.; Carter, B. S. PloS one 2016, 11, 1-11.

[157] Koritzinsky, E.; Street, J.; Star, R.; Yuen, P. J. Cell. Physio. 2016, 9-18.

[158] Mørk, M.; Pedersen, S.; Botha, J.; Lund, S. M.; Kristensen, S. R. Scand. J. Clin. Lab. Invest. 2016, 76, 349-360.

[159] Shimbo, K.; Miyaki, S.; Ishitobi, H.; Kato, Y.; Kubo, T.; Shimose, S.; Ochi, M. Biochem. Biophys. Res. Commun. 2014, 445, 381-387.

[160] Cheng, L.; Sharples, R. A.; Scicluna, B. J.; Hill, A. F. J. Extracell. Vesicles 2014, 3, 23743.

[161] Vogel, R.; Coumans, F. A.; Maltesen, R. G.; Böing, A. N.; Bonnington, K. E.; Broekman, M. L.; Broom, M. F.; Buzás, E. I.; Christiansen, G.; Hajji, N., et al. J. Extracell. Vesicles 2016, 5, DOI: 10.3402 / jev . v5.31242. 
[162] Szabó, G. T.; Tarr, B.; Pálóczi, K.; Éder, K.; Lajkó, E.; Kittel, Á.; Tóth, S.; György, B.; Pásztói, M.; Németh, A., et al. Cell. Mol. Life Sci. 2014, 4055-4067.

[163] Anderson, W.; Lane, R.; Korbie, D.; Trau, M. Langmuir 2015, 31, $6577-6587$.

[164] Cheng, L.; Sun, X.; Scicluna, B. J.; Coleman, B. M.; Hill, A. F. Kidney Int. 2014, 86, 433-444.

[165] Connolly, K. D.; Willis, G. R.; Datta, D. B.; Ellins, E. A.; Ladell, K.; Price, D. A.; Guschina, I. A.; Rees, D. A.; James, P. E. J. Lipid Res. 2014, 55, 2064-2072.

[166] Chou, M.-L.; Lin, L.-T.; Devos, D.; Burnouf, T. Transfusion 2015, 55, $2433-2444$.

[167] Lin, H.-C.; Chang, H.-W.; Hsiao, S.-H.; Chou, M.-L.; Seghatchian, J.; Burnouf, T. Transfus. Apher. Sci. 2015, 53, 246-252.

[168] György, B.; Szabó, T. G.; Pásztói, M.; Pál, Z.; Misják, P.; Aradi, B.; László, V.; Pállinger, E.; Pap, E.; Kittel, A., et al. Cell. Mol. Life Sci. 2011, 68, 2667-2688.

[169] Lee, Y.; Andaloussi, S. E.; Wood, M. J. Hum. Mol. Gen. 2012, 21, 125134. 
[170] De Vrij, J.; Maas, S.; Kwappenberg, K.; Schnoor, R.; Kleijn, A.; Dekker, L.; Luider, T. M.; de Witte, L. D.; Litjens, M.; van Strien, M. E., et al. Int. J. Cancer 2015, 137, 1630-1642.

[171] Yamashita, T.; Takahashi, Y.; Nishikawa, M.; Takakura, Y. Eur. J. Pharm. Biopharm. 2016, 98, 1-8.

[172] Katsuda, T.; Tsuchiya, R.; Kosaka, N.; Yoshioka, Y.; Takagaki, K.; Oki, K.; Takeshita, F.; Sakai, Y.; Kuroda, M.; Ochiya, T. Sci. Rep. 2013, $3,1197$.

[173] Nakamura, Y.; Miyaki, S.; Ishitobi, H.; Matsuyama, S.; Nakasa, T.; Kamei, N.; Akimoto, T.; Higashi, Y.; Ochi, M. FEBS Lett. 2015, 589, 1257-1265.

[174] Zhang, Y.; Chopp, M.; Meng, Y.; Katakowski, M.; Xin, H.; Mahmood, A.; Xiong, Y. J. Neurosurg. 2015, 122, 856-867.

[175] Ng, Y. H.; Rome, S.; Jalabert, A.; Forterre, A.; Singh, H.; Hincks, C. L.; Salamonsen, L. A. PloS one 2013, 8, e58502.

[176] Chang, H.-. I.; Yeh, M.-K. Int. J. Nanomedicine 2012, 7, 49-60.

[177] Bogomolny, E.; Hong, J.; Blenkiron, C.; Simonov, D.; Dauros, P.; Swift, S.; Phillips, A.; Willmott, G. R. SPIE BiOS 2015, DOI: 10 . 
[178] Choi, D. H.; Kwon, Y. M.; Chiura, H. X.; Yang, E. C.; Bae, S. S.; Kang, S. G.; Lee, J.-H.; Yoon, H. S.; Kim, S.-J. Appl. Environ. Microbiol. 2015, $81,4591-4599$.

[179] Abstracts from the Third International Meeting of ISEV 2014 Rotterdam, The Netherlands, April 30th - May 3rd, 2014., Vol. 3, 2014.

[180] Connolly, K. Adipocyte-derived extracellular vesicles: characterisation and function., Ph.D. Thesis, Cardiff University, 2016.

[181] Polanco, J. C.; Scicluna, B. J.; Hill, A. F.; Götz, J. J. Biol. Chem. 2016, $291,12445-12466$.

[182] Lammers, T.; Kiessling, F.; Hennink, W. E.; Storm, G. J. Control. Release 2012, 161, 175-187.

[183] Davis, M. E.; Shin, D. M., et al. Nat. Rev. Drug Discov. 2008, 7, 771782.

[184] Torchilin, V. P. Pharm. Res. 2007, 24, 1-16.

[185] Garza-Licudine, E.; Deo, D.; Yu, S.; Uz-Zaman, A.; Dunbar, W. B. Annual International Conference of the IEEE Engineering in Medicine and Biology 2010, 5736-5739. 
[186] Liu, H.; Maruyama, H.; Masuda, T.; Arai, F. Sensor Actuat. B Chem. $2015,220,40-49$.

[187] Van Bracht, E.; Raavé, R.; Verdurmen, W. P.; Wismans, R. G.; Geutjes, P. J.; Brock, R. E.; Oosterwijk, E.; van Kuppevelt, T. H.; Daamen, W. F. Int. J. Pharm. 2012, 439, 127-135.

[188] Burgess, M. T.; Porter, T. Proceedings of Meeting on Acoustics 2013, 19, 075059 .

[189] Burgess, M. T.; Porter, T. M. Ultrasound Med. Biol. 2015, 41, 21912201.

[190] Ruff, L. E.; Mahmoud, E. A.; Sankaranarayanan, J.; Morachis, J. M.; Katayama, C. D.; Corr, M.; Hedrick, S. M.; Almutairi, A. Integr. Biol. 2013, 5, 195-203.

[191] Naeem, M.; Kim, W.; Cao, J.; Jung, Y.; Yoo, J.-W. Colloids Surf. B 2014, $123,271-278$.

[192] Colby, A.; Colson, Y.; Grinstaff, M. W. Nanoscale 2013, 5, 3496-3504.

[193] Stolzoff, M.; Ekladious, I.; Colby, A. H.; Colson, Y. L.; Porter, T. M.; Grinstaff, M. W. Biomacromolecules 2015, 16, 1958-1966. 
[194] Pevarnik, M.; Schiel, M.; Yoshimatsu, K.; Vlassiouk, I. V.; Kwon, J. S.; Shea, K. J.; Siwy, Z. S. ACS Nano 2013, 7, 3720-3728.

[195] Holden, D. A.; Watkins, J. J.; White, H. S. Langmuir 2012, 28, 75727577.

[196] Bachman, H.; Brown, A. C.; Clarke, K. C.; Dhada, K. S.; Douglas, A.; Hansen, C. E.; Herman, E.; Hyatt, J. S.; Kodlekere, P.; Meng, Z., et al. Soft Matter 2015, 11, 2018-2028.

[197] Kodlekere, P.; Cartelle, A. L.; Lyon, L. A. RSC Adv. 2016, 6, 3161931631.

[198] Nurhasni, H.; Cao, J.; Choi, M.; Kim, I.; Lee, B. L.; Jung, Y.; Yoo, J.-W. Int. J. Nanomedicine 2015, 10, 3065-3080.

[199] Cao, J.; Naeem, M.; Noh, J.-K.; Lee, E. H.; Yoo, J.-W. Macromol. Res. 2015, 23, 485-492.

[200] Hwang, T.-L.; Aljuffali, I. A.; Lin, C.-F.; Chang, Y.-T.; Fang, J.-Y. Int. J. Nanomedicine 2015, 10, 371-385.

[201] Amirfazli, A. Nat. Nanotechnol. 2007, 2, 467-468.

[202] Upadhyay, D.; Scalia, S.; Vogel, R.; Wheate, N.; Salama, R. O.; Young, P. M.; Traini, D.; Chrzanowski, W. Pharm. Res. 2012, 29, 2456-2467. 
[203] Astafyeva, K. Physical and acoustical properties of fluorocarbon nanoparticles., PhD Thesis, Université Pierre et Marie Curie, 2014.

[204] Astafyeva, K.; Somaglino, L.; Desgranges, S.; Berti, R.; Patinote, C.; Langevin, D.; Lazeyras, F.; Salomir, R.; Polidori, A.; Contino-Pepin, C., et al. J. Mater. Chem. B 2015, 3, 2892-2907.

[205] Manta, S.; Delalande, A.; Bessodes, M.; Bureau, M. F.; Scherman, D.; Pichon, C.; Mignet, N. Ultrasound Med. Biol. 2016, 42, 624-630.

[206] Pal, A. K.; Watson, C. Y.; Pirela, S. V.; Singh, D.; Chalbot, M.-C. G.; Kavouras, I.; Demokritou, P. Toxicol. Sci 2015, 146, 321-333.

[207] Rybakova, D.; Radjainia, M.; Turner, A.; Sen, A.; Mitra, A. K.; Hurst, M. R. H. Mol. Microbiol. 2013, 89, 702-714.

[208] Akpinar, F.; Yin, J. J. Virol. Methods 2015, 218, 71-76.

[209] Gaudin, R.; Barteneva, N. S. Nat. Commun. 2015, 6, DOI: 10.1038 / ncomms 7022.

[210] Bennett, G.; Rajan, R.; Bunt, C. R.; Hussain, M. A. NZ Vet. J. 2012, $61,119-120$.

[211] Pang, L.; Nowostawska, U.; Weaver, L.; Hoffman, G.; Karmacharya, A.; Skinner, A.; Karki, N. Environ. Sci. Technol. 2012, 46, 11779-11787. 
[212] Saqer, L.; Al Ahmad, M.; Taher, H.; Al-Zuhair, S.; Al Naqbi, A. H. GCCCE IEEE 2015, DOI: 10.1109 / IEEEGCC . 2015.7060075.

[213] Pankhurst, Q. A.; Connolly, J.; Jones, S.; Dobson, J. J. Phys. D: Appl. 2003, 36, R167.

[214] Buchs, B.; Evangelou, M. W. H.; Winkel, L. H. E.; Lenz, M. Environ. Sci. Technol. 2013, 47, 2401-2407.

[215] Yoon, J.; Kota, A.; Bhaskar, S.; Tuteja, A.; Lahann, J. ACS Appl. Mater. Interfaces 2013, 5, 11281-11287.

[216] Saxena, S.; Lyon, L. A. J. Coll. Interf. Sci. 2015, 442, 39-48.

[217] Bangs Laboratories Inc., Ask the Particle Doctor., http : / / www . bangslabs.com/sites/default/files/imce/docs/ParticleDrQandA. pdf, 2015 (accessed September 1, 2015).

[218] Fairley, N. CasaXPS Version 2.3.14., https : / / www . casaxps . com, 1999-2016.

[219] Rasband, W. S. ImageJ; National Institutes of Health: Bethesda, Maryland., https: //imagej.nih.gov/ij/, 1997-2016.

[220] Blundell, E. L. C. J.; Vogel, R.; Platt, M. Langmuir 2016, 32, 10821090. 
[221] Hunter, R. J., Zeta Potential in Colloid Science; Academic Press, London: 1981.

[222] Ohsawa, K.; Murata, M.; Ohshima, H. Colloid Polym. Sci. 1986, 264, 1005-1009.

[223] Kirby, B. J.; Hasselbrink, E. F. Electrophoresis 2004, 25, 187-202.

[224] Zetasizer Nano accessories guide., http://www.cif.iastate. edu/sites/default/files/uploads/Other_Inst/ParticleSize/ AccessoriesandCellsGuide.pdf, 2013 (accessed October 27, 2016).

[225] Lefèvre, G.; Čerović, L.; Milonjić, S.; Fédoroff, M.; Finne, J.; Jaubertie, A. J. Coll. Interf. Sci. 2009, 337, 449-455.

[226] Burgreen, D.; Nakache, F. J. Phys. Chem. 1964, 68, 1084-1091.

[227] Rice, C.; Whitehead, R. J. Phys. Chem. 1965, 69, 4017-4024.

[228] Cummings, E. B.; Griffiths, S.; Nilson, R.; Paul, P. Anal. Chem. 2000, $72,2526-2532$.

[229] Möckel, D.; Staude, E.; Dal-Cin, M.; Darcovich, K.; Guiver, M. J. Membr. Sci. 1998, 145, 211-222. 
[230] Nyström, M.; Lindström, M.; Matthiasson, E. Colloids Surf. 1989, 36, 297-312.

[231] Sun, L.; Crooks, R. M. J. Am. Chem. Soc. 2000, 122, 12340-12345.

[232] Sun, J.; Kang, Y.; Boczko, E. M.; Jiang, X. Electroanalysis 2013, 25, $1023-1028$.

[233] Cervera, J.; Ramírez, P.; Manzanares, J. A.; Mafé, S. Microfluid. Nanofluidics 2010, 9, 41-53.

[234] Anderson, W.; Vogel, R.; Trau, M. Single nano micropore $\zeta$-potential measurements., Poster, International Conference on BioNano Innovation, University of Queensland, 2015.

[235] Innes, L.; Gutierrez, D.; Mann, W.; Buchsbaum, S. F.; Siwy, Z. S. Analyst 2015, 140, 4804-4812.

[236] Weatherall, E.; Willmott, G. R. J. Phys. Chem. B 2015, 119, 5328-5335.

[237] Kosmulski, M. J. Coll. Interf. Sci. 2011, 353, 1-15.

[238] Pardon, G.; Gatty, H. K.; Stemme, G.; van der Wijngaart, W.; Roxhed, N. Nanotechnology 2012, 24, 1-11.

[239] Xie, Y.; Xue, J.; Wang, L.; Wang, X.; Jin, K.; Chen, L.; Wang, Y. Langmuir 2009, 25, 8870-8874. 
[240] Pérez-Mitta, G.; Tuninetti, J. S.; Knoll, W.; Trautmann, C.; ToimilMolares, M. E.; Azzaroni, O. J. Am. Chem. Soc. 2015, 137, 6011-6017.

[241] Nguyen, G.; Howorka, S.; Siwy, Z. S. J. Membr. Biol. 2011, 239, 105113.

[242] De Groot, G. W.; Santonicola, M. G.; Sugihara, K.; Zambelli, T.; Reimhult, E.; Vörös, J.; Vancso, G. J. ACS Appl. Mater. Interfaces 2013, $5,1400-1407$.

[243] Bressan, R. A.; Crippa, J. A. Acta Psychiat. Scand. 2005, 111, 14-21.

[244] Lee, H.; Scherer, N. F.; Messersmith, P. B. Proc. Natl. Acad. Sci. 2006, 103, 12999-13003.

[245] Lee, H.; Dellatore, S. M.; Miller, W. M.; Messersmith, P. B. Science $2007,318,426-430$.

[246] Bandara, N.; Zeng, H.; Wu, J. J. Adhes. Sci Technol. 2013, 27, 21392162.

[247] Liu, Y.; Ai, K.; Lu, L. Chem. Rev. 2014, 114, 5057-5115.

[248] Lynge, M. E.; van der Westen, R.; Postma, A.; Städler, B. Nanoscale 2011, 3, 4916-4928. 
[249] Ejima, H.; Richardson, J. J.; Liang, K.; Best, J. P.; van Koeverden, M. P.; Such, G. K.; Cui, J.; Caruso, F. Science 2013, 341, 154-157.

[250] Bueno-Ferrer, C.; Hablot, E.; Perrin-Sarazin, F.; Garrigós, M. C.; Jiménez, A.; Averous, L. Macromol. Mater. Eng. 2012, 297, 777-784.

[251] Zangmeister, R. A.; Morris, T. A.; Tarlov, M. J. Langmuir 2013, 29, 8619-8628.

[252] Amstad, E.; Gehring, A. U.; Fischer, H.; Nagaiyanallur, V. V.; Hähner, G.; Textor, M.; Reimhult, E. J. Phys. Chem. C 2010, 115, 683-691.

[253] Weibel, D. E.; Vilani, C.; Habert, A. C.; Achete, C. A. J. Membr. Sci. 2007, 293, 124-132.

[254] Brandrup, J.; Immergut, E. H.; Grulke, E. A.; Abe, A.; Bloch, D. R., Polymer Handbook; John Wiley \& Sons, New York: 1989; Vol. 7.

[255] Moulder, J. F., Handbook of X-ray photoelectron spectroscopy: a reference book of standard spectra for identification and interpretation of XPS data; Chastain, J., Ed.; Physical Electronics Division, Perkin-Elmer Corporation: 1995.

[256] Siwy, Z. S. Adv. Funct. Mater. 2006, 16, 735-746. 
[257] Siwy, Z.; Apel, P.; Baur, D.; Dobrev, D. D.; Korchev, Y. E.; Neumann, R.; Spohr, R.; Trautmann, C.; Voss, K.-O. Surf. Sci. 2003, 532, 10611066.

[258] Apel, P. Y.; Korchev, Y. E.; Siwy, Z.; Spohr, R.; Yoshida, M. Nucl. Instr. Meth. Phys. Res. B 2001, 184, 337-346.

[259] Siwy, Z.; Gu, Y.; Spohr, H. A.; Baur, D.; Wolf-Reber, A.; Spohr, R.; Apel, P.; Korchev, Y. E. Europhys. Lett. 2002, 60, 349-355.

[260] Lan, W.-J.; Holden, D. A.; White, H. S. J. Am. Chem. Soc. 2011, 133, 13300-13303.

[261] Mévellec, V.; Roussel, S.; Tessier, L.; Chancolon, J.; Mayne-L'Hermite, M.; Deniau, G.; Viel, P.; Palacin, S. Chem. Mater. 2007, 19, 6323-6330.

[262] Brisset, F.; Vieillard, J.; Berton, B.; Morin-Grognet, S.; DuclairoirPoc, C.; Le Derf, F. Appl. Surf. Sci. 2015, 329, 337-346.

[263] Decher, G. Science 1997, 277, 1232-1237.

[264] Hammond, P. T. Adv. Mater. 2004, 16, 1271-1293.

[265] Tang, Z.; Wang, Y.; Podsiadlo, P.; Kotov, N. A. Adv. Mater. 2006, 18, 3203-3224. 
[266] Powell, M. R.; Sullivan, M.; Vlassiouk, I.; Constantin, D.; Sudre, O.; Martens, C. C.; Eisenberg, R. S.; Siwy, Z. S. Nat. Nanotechnol. 2008, 3, 51-57.

[267] Chang, H.; Kosari, F.; Andreadakis, G.; Alam, M. A.; Vasmatzis, G.; Bashir, R. Nano Lett. 2004, 4, 1551-1556.

[268] Chang, H.; Venkatesan, B. M.; Iqbal, S. M.; Andreadakis, G.; Kosari, F.; Vasmatzis, G.; Peroulis, D.; Bashir, R. Biomed. Microdevices 2006, 8, 263-269.

[269] Smeets, R. M. M.; Keyser, U. F.; Krapf, D.; Wu, M.-Y.; Dekker, N. H.; Dekker, C. Nano Lett. 2006, 6, 89-95.

[270] Heng, J. B.; Ho, C.; Kim, T.; Timp, R.; Aksimentiev, A.; Grinkova, Y. V.; Sligar, S.; Schulten, K.; Timp, G. Biophys. J. 2004, 87, 2905-2911.

[271] Kowalczyk, S. W.; Dekker, C. Nano Lett. 2012, 12, 4159-4163.

[272] Fan, R.; Karnik, R.; Yue, M.; Li, D.; Majumdar, A.; Yang, P. Nano Lett. 2005, 5, 1633-1637.

[273] Maffeo, C.; Yoo, J.; Comer, J.; Wells, D. B.; Luan, B.; Aksimentiev, A. J. Phys. Condens. Matter 2014, 26, 413101. 
[274] Shankla, M.; Aksimentiev, A. Nat. Commun. 2014, 5, DOI: 10.1038 / ncomms 6171.

[275] Goyal, G.; Freedman, K. J.; Kim, M. J. Anal. Chem. 2013, 85, 81808187.

[276] Wang, Y.; Kececi, K.; Mirkin, M. V.; Mani, V.; Sardesai, N.; Rusling, J. F. Chem. Sci. 2013, 4, 655-663.

[277] Menestrina, J.; Yang, C.; Schiel, M.; Vlassiouk, I. V.; Siwy, Z. S. J. Phys. Chem. C 2014, 118, 2391-2398.

[278] Bangs Laboratories Inc., TechNote 206., https : / / www . bangs labs . com/sites/default/files/imce/docs/TechNote206Web. pdf, 2001 (accessed October 13, 2015).

[279] Wright, M. R., An Introduction to Aqueous Electrolyte Solutions; John Wiley \& Sons, West Sussex: 2007.

[280] Smythe, W. Rev. Sci. Instrum. 1972, 43, 817-818.

[281] Qin, Z.; Zhe, J.; Wang, G.-X. Meas. Sci. Technol. 2011, 22, 045804.

[282] Tsutsui, M.; He, Y.; Yokota, K.; Arima, A.; Hongo, S.; Taniguchi, M.; Washio, T.; Kawai, T. ACS Nano 2015, 10, 803-809.

[283] Berge, L. I.; Jossang, T.; Feder, J. Meas. Sci. Technol. 1990, 1, 471-474. 
[284] Saleh, O.; Sohn, L. Rev. Sci. Instrum. 2002, 73, 4396-4398.

[285] Weatherall, E.; Hauer, P.; Vogel, R.; Willmott, G. R. Anal. Chem. 2016, 88, 8648-8656.

[286] Di Carlo, D. Lab Chip 2009, 9, 3038-3046.

[287] Martel, J. M.; Toner, M. Annu. Rev. Biomed. Eng. 2014, 16, 371-396.

[288] Shank, B. B.; Adams, R. B.; David, K. J. Lab. Clin. Med. 1969, 74, 630641. 
Appendices 



\section{Appendix A}

\section{Publications and Presentations}

\section{A.1 Publications}

- Weatherall, E.; Willmott, G. R. \& Glossop, B. Individual nanoparticle zeta potential measurements using tunable resistive pulse sensing. Seventh International Conference on Sensing Technology (ICST), 2013, 874-878

- Weatherall, E. \& Willmott, G. R. Conductive and Biphasic Pulses in Tunable Resistive Pulse Sensing. J. Phys. Chem. B, ACS Publications, $2015,119,5328-5335$ 
- Weatherall, E. \& Willmott, G. R. Applications of tunable resistive pulse sensing. Analyst, Royal Society of Chemistry, 2015, 140, 33183334

- Weatherall, E.; Hauer, P.; Vogel, R. \& Willmott, G. R. Pulse Size Distributions in Tunable Resistive Pulse Sensing. Anal. Chem., ACS Publications, 2016, 88, 8648-8656

\section{A.2 Patents}

- Glossop, B. M.; Vogel, R.; Weatherall, E. \& Jones, M. D. "Measurement of particle charge." U.S. Patent Application No. 14/916,405.

\section{A.3 Conference Presentations}

- Weatherall, E.; Willmott, G. R. \& Glossop, B. "Theory and Applications of Charge Analysis using TRPS Systems" Talk presented at Izon Science Research Symposium. Boston, USA, October 2013.

- Weatherall, E.; Willmott, G. R. \& Glossop, B. "Individual nanoparticle zeta potential measurements using tunable resistive pulse sens- 
ing" Poster presented at Seventh International Conference on Sensing Technology (ICST). Wellington, New Zealand, December 2013.

- Weatherall, E. \& Willmott, G. R. "Current Enhancement in Tunable Resistive Pulse Sensing at Low Electrolyte Concentration" Talk presented at Fifth Australia and New Zealand Nano- and Micro-fluidics (ANZNMF) Symposium and Workshop. Hobart, Australia, April 2014.

- Weatherall, E. \& Willmott, G. R. "Tunable resistive pulse sensing" Talk presented at The MacDiarmid Institute Student \& Postdoc Symposium. Auckland, New Zealand, November 2014.

- Weatherall, E. \& Willmott, G. R. "Particle charge measurement using tunable resistive pulse sensing" Talk presented at Seventh International Conference on Advanced Materials and Nanotechnology (AMN-7), Nelson, New Zealand, February 2015.

- Weatherall, E. \& Willmott, G. R. "Conductive and Biphasic Pulses in Tunable Resistive Pulse Sensing" Poster presented at The MacDiarmid Institute Student \& Postdoc Symposium. Wellington, New Zealand, November 2015. 
- Weatherall, E. \& Willmott, G. R. "Particle and pore zeta-potential measurements using tunable resistive pulse sensing" Poster presented at EMBL Conference: Microfluidics 2016. Heidelberg, Germany, July 2016. 


\section{Appendix B}

\section{SEM Images}

A20407

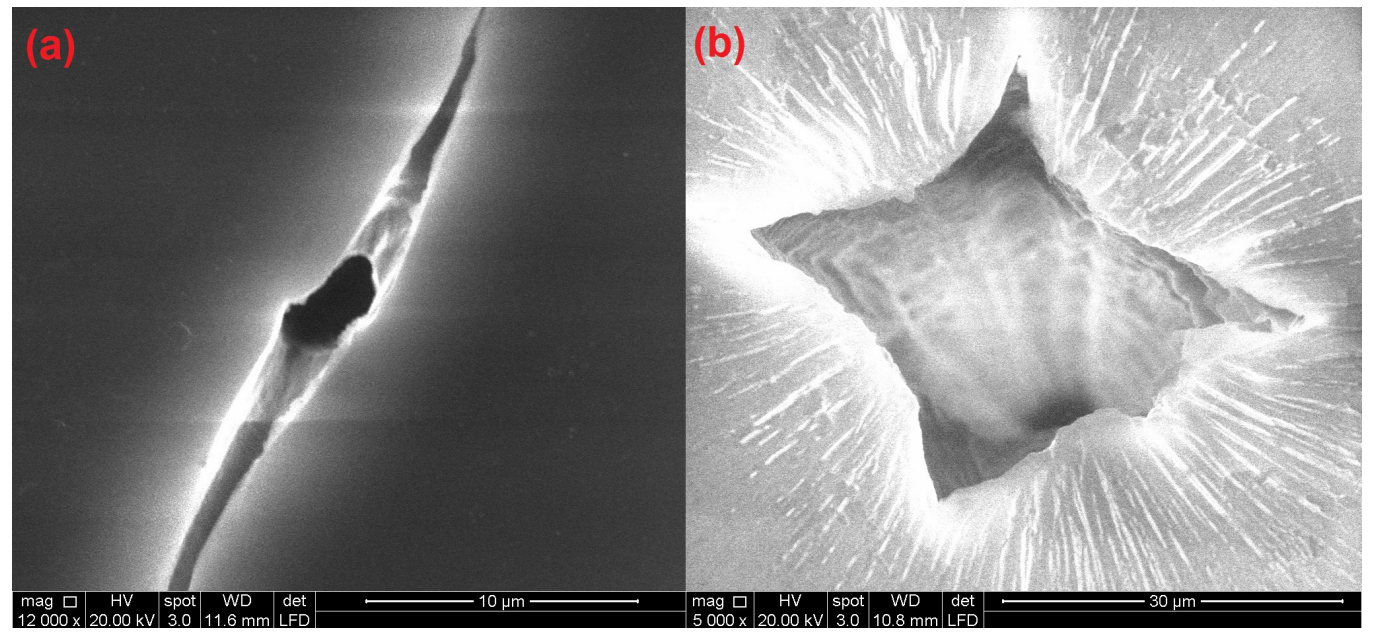

Figure B.1: SEM images of (a) small pore opening and (b) large pore opening for NP150 pore A20407 


\section{A21820}

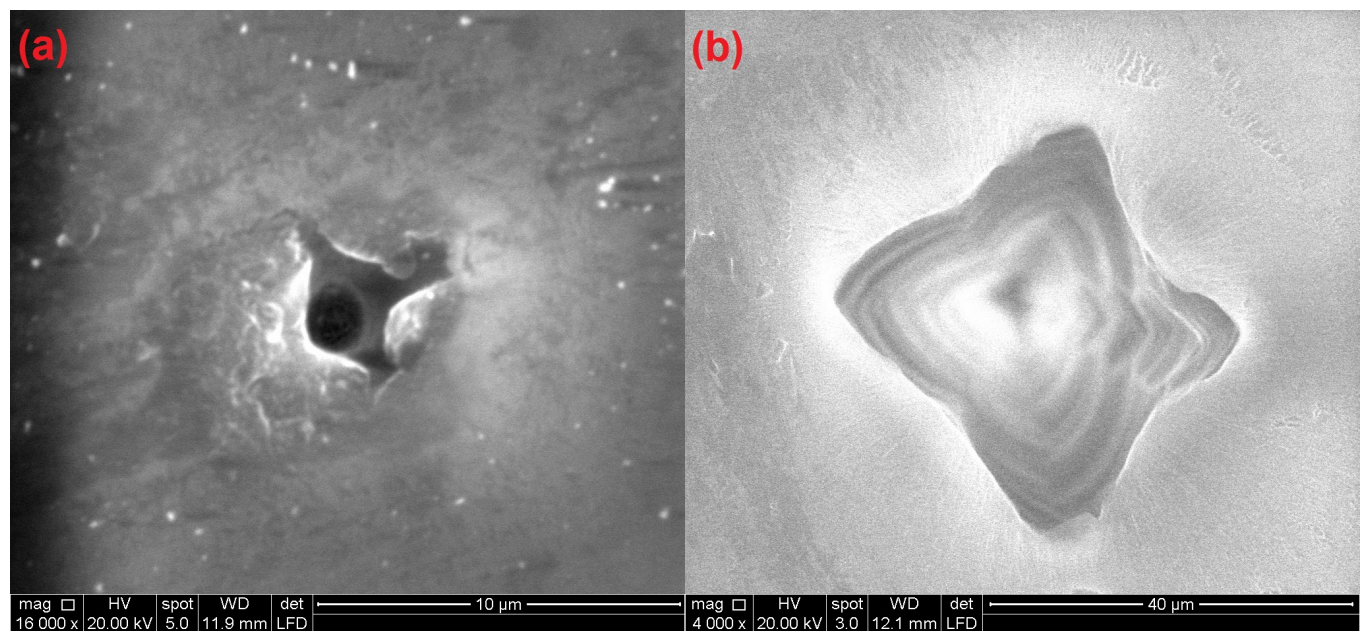

Figure B.2: SEM images of (a) small pore opening and (b) large pore opening for NP150 pore A21820

\section{A28818}

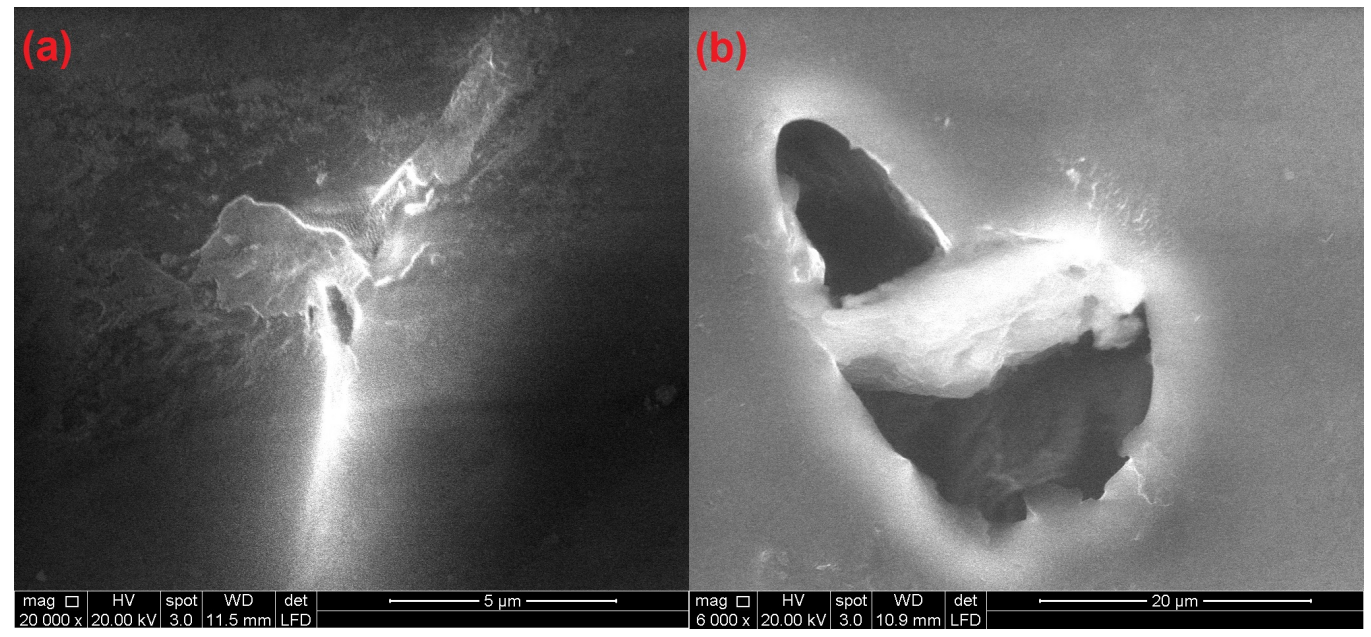

Figure B.3: SEM images of (a) small pore opening and (b) large pore opening for NP150 pore A28818 


\section{A32029}

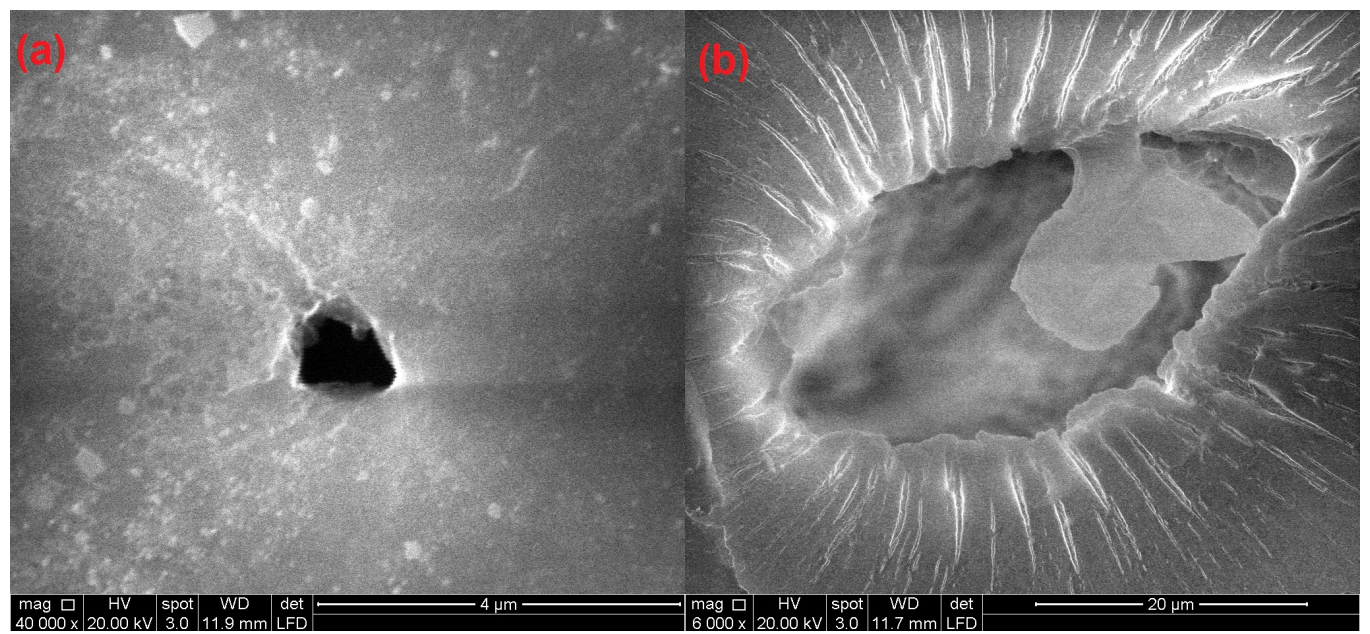

Figure B.4: SEM images of (a) small pore opening and (b) large pore opening for NP200 pore A32029

\section{A12353}

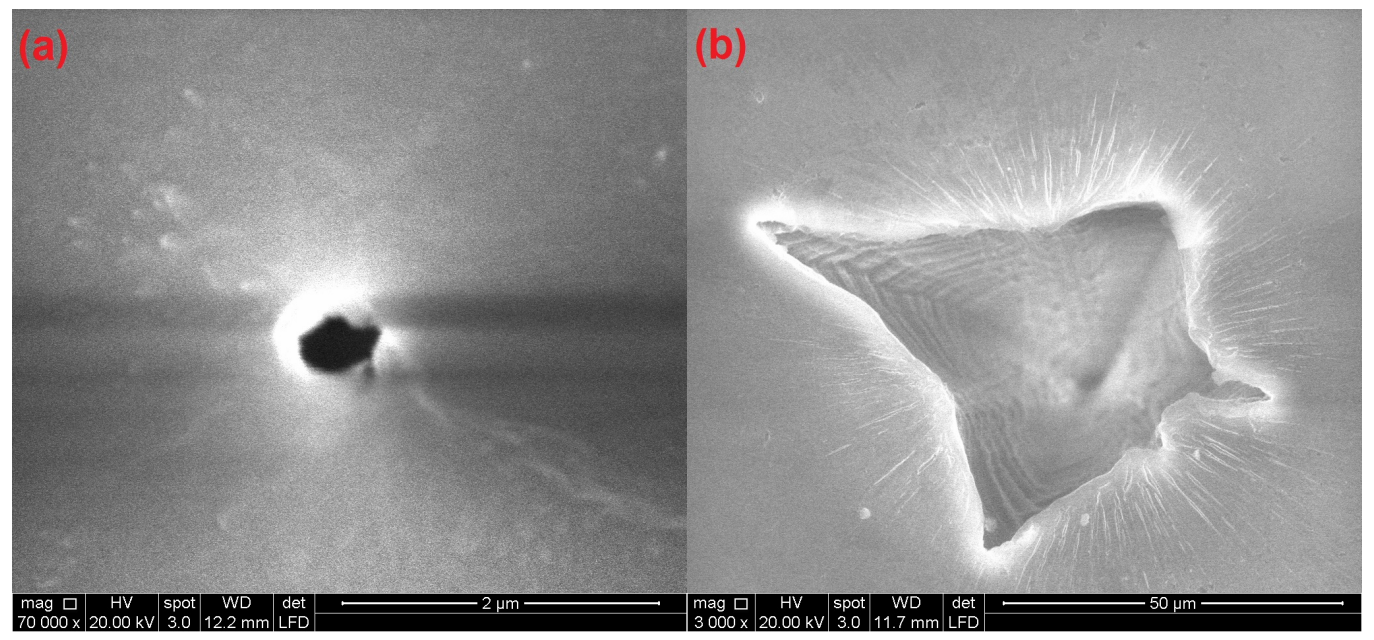

Figure B.5: SEM images of (a) small pore opening and (b) large pore opening for NP400 pore A12353 


\section{A22300}

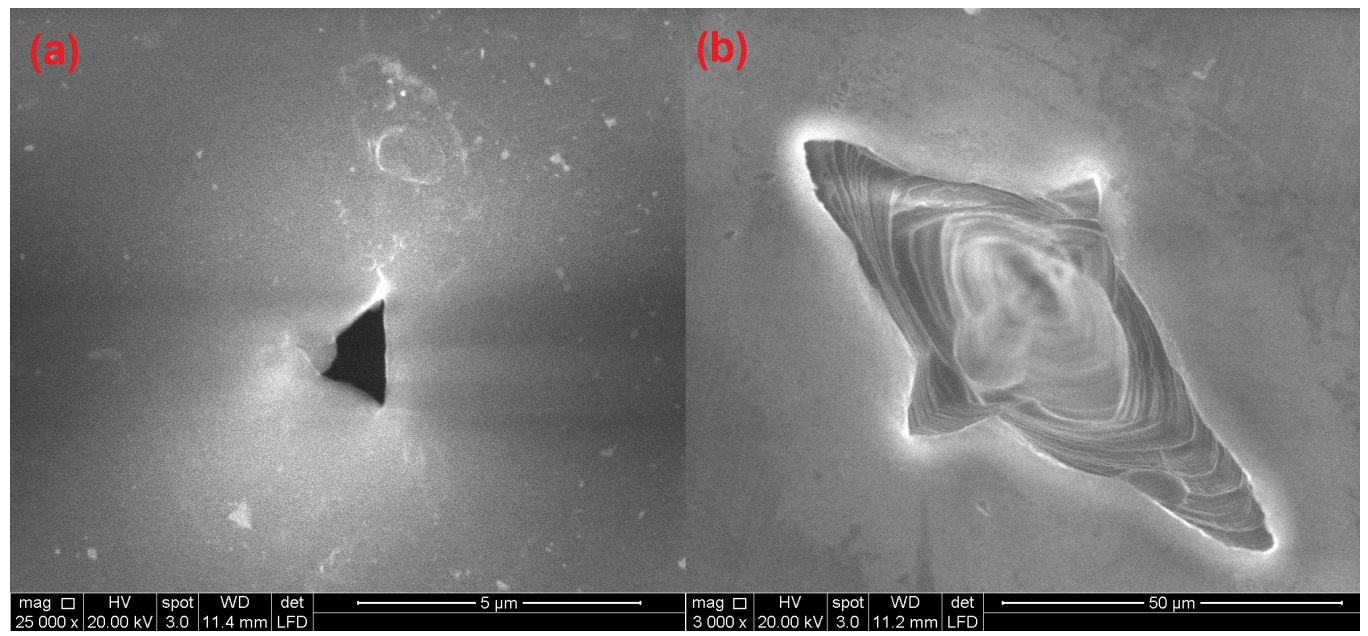

Figure B.6: SEM images of (a) small pore opening and (b) large pore opening for NP800 pore A22300

A40357

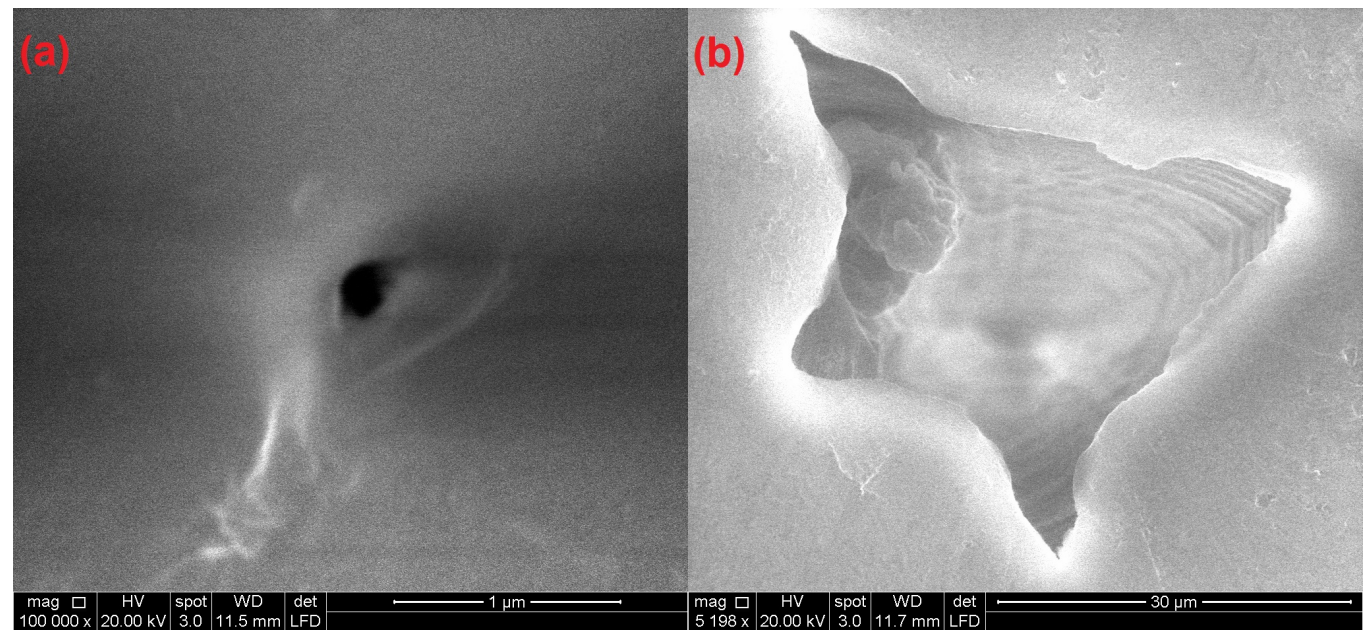

Figure B.7: SEM images of (a) small pore opening and (b) large pore opening for NP150 pore A40357 


\section{A40338}

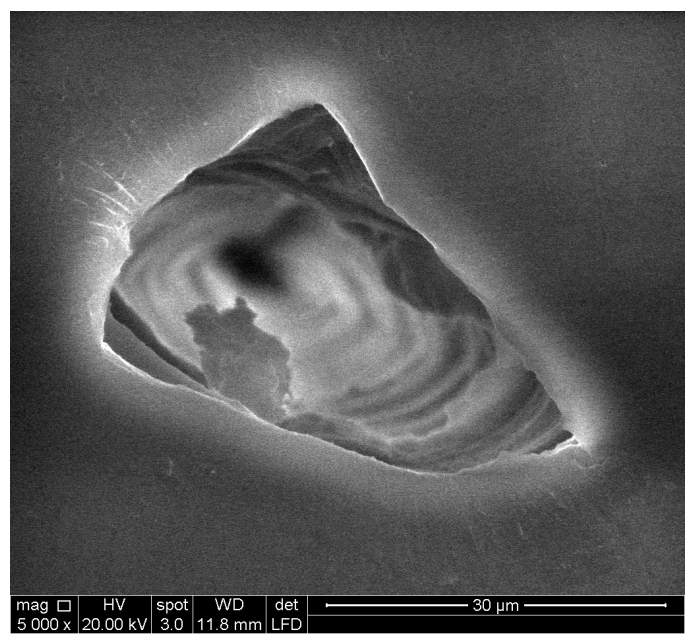

Figure B.8: SEM image of the large pore opening for NP150 pore A40338

A41972

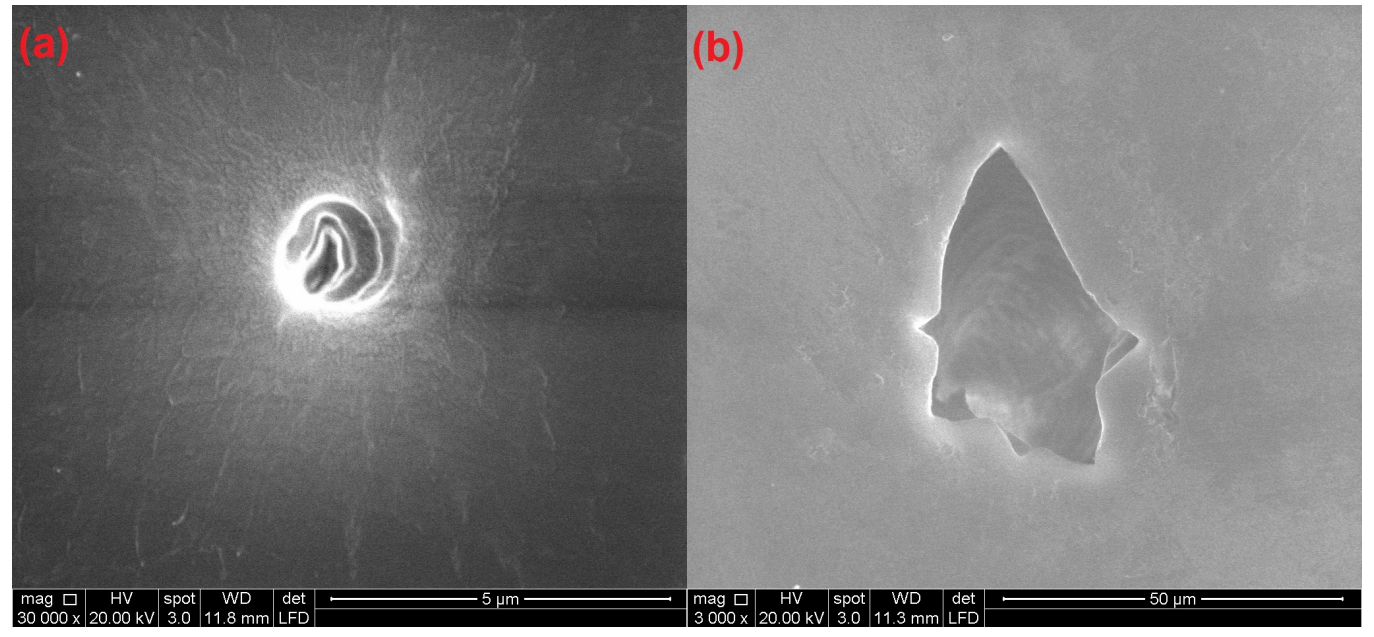

Figure B.9: SEM images of (a) small pore opening and (b) large pore opening for NP150 pore A41972 


\section{NHH23}

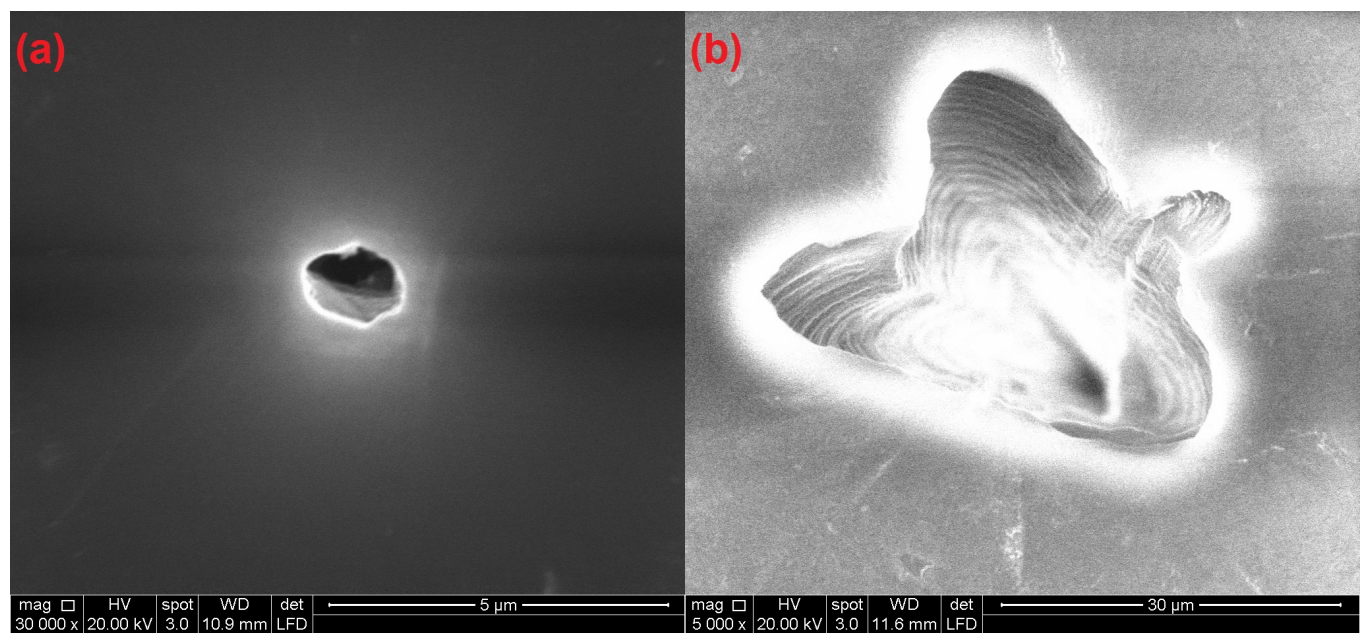

Figure B.10: SEM images of (a) small pore opening and (b) large pore opening for NP2000 pore NHH23

A21032

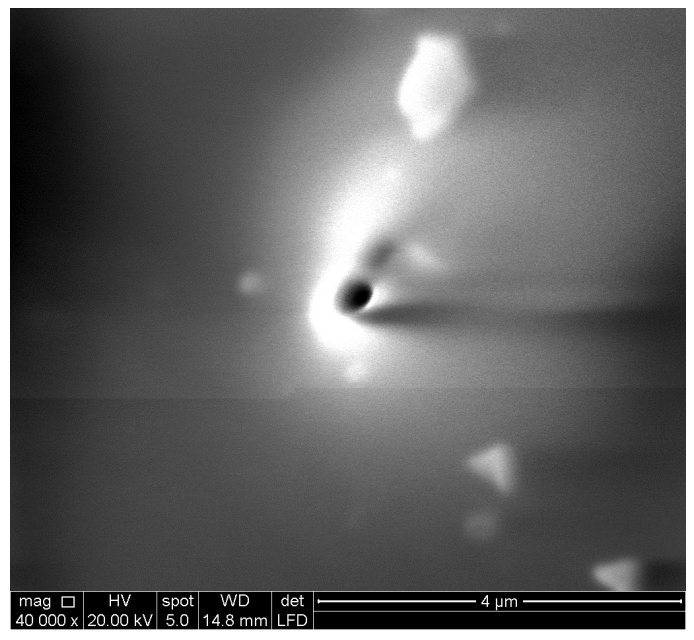

Figure B.11: SEM image of small pore opening for NP150 pore A21032 


\section{A23802}

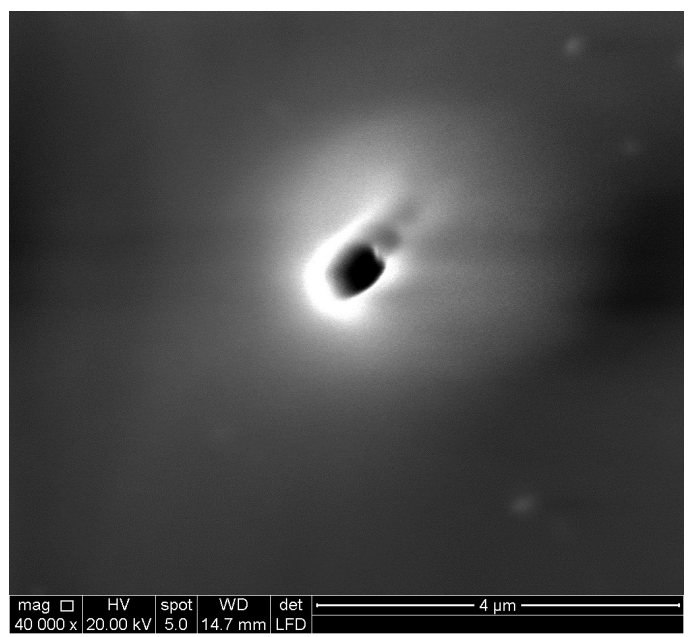

Figure B.12: SEM image of small pore opening for NP150 pore A23802

A25135

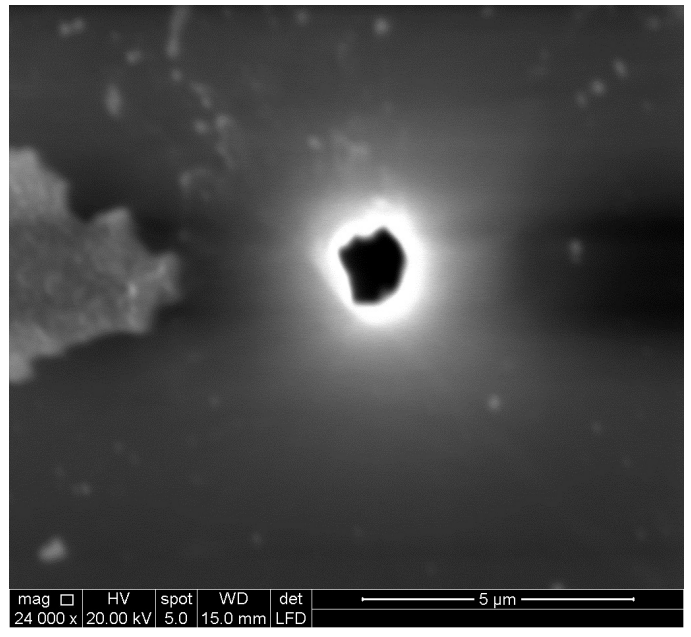

Figure B.13: SEM image of small pore opening for NP150 pore A25135 
A27082

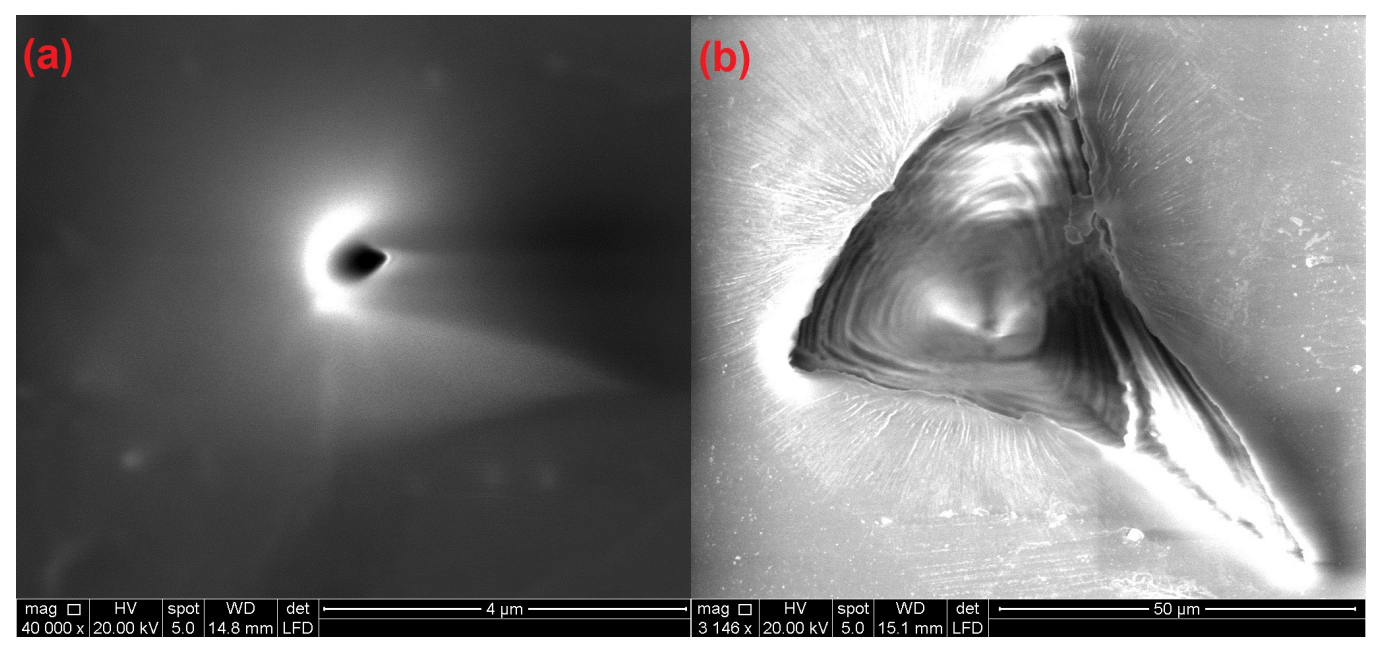

Figure B.14: SEM images of (a) small pore opening and (b) large pore opening for NP150 pore A27082

A25136

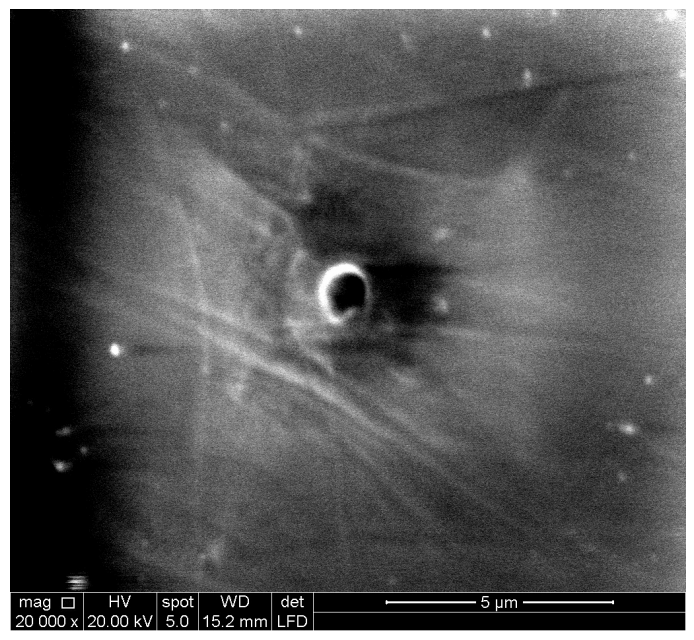

Figure B.15: SEM image of small pore opening for NP150 pore A25136 


\section{A33701}

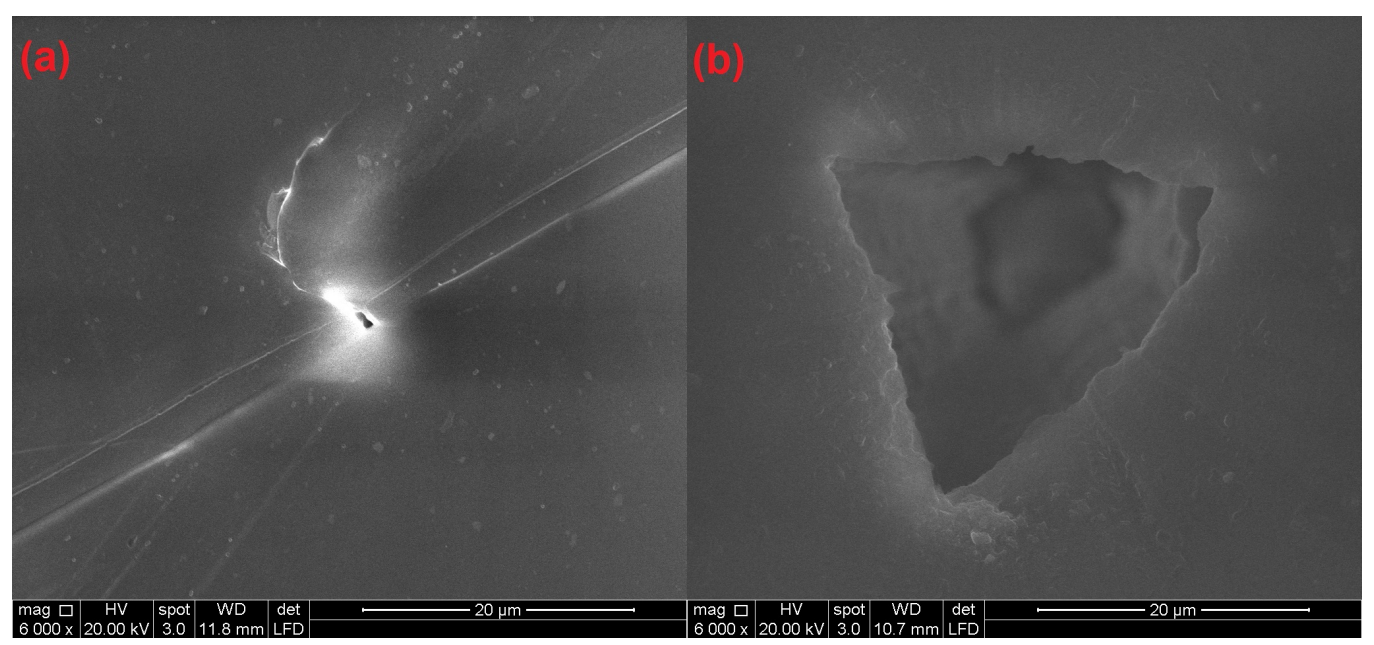

Figure B.16: SEM images of (a) small pore opening and (b) large pore opening for NP150 pore A33701 
Appendix C

$\zeta$-potential Raw Data Example:

Method IV 


\begin{tabular}{|r|r|r|r|r|r|r|r|r|r|r|r|r|r|}
\hline $\mathbf{0 . 0 5}$ & $\mathbf{0 . 1}$ & $\mathbf{0 . 1 5}$ & $\mathbf{0 . 2}$ & $\mathbf{0 . 2 5}$ & $\mathbf{0 . 3}$ & $\mathbf{0 . 3 5}$ & $\mathbf{0 . 4}$ & $\mathbf{0 . 4 5}$ & $\mathbf{0 . 5}$ & $\mathbf{0 . 6}$ & $\mathbf{0 . 7}$ & $\mathbf{0 . 8}$ & $\mathbf{0 . 9}$ \\
\hline 2.198 & 1.406 & 0.941 & 0.666 & 0.495 & 0.374 & 0.315 & 0.275 & 0.233 & 0.202 & 0.154 & 0.119 & 0.08 & 0.044 \\
\hline 2.565 & 1.836 & 0.688 & 0.55 & 0.412 & 0.341 & 0.286 & 0.258 & 0.229 & 0.202 & 0.161 & 0.133 & 0.104 & 0.061 \\
\hline 2.1 & 1.224 & 0.987 & 0.671 & 0.532 & 0.394 & 0.36 & 0.311 & 0.277 & 0.252 & 0.171 & 0.137 & 0.109 & 0.068 \\
\hline 1.834 & 1.207 & 0.9 & 0.64 & 0.451 & 0.38 & 0.327 & 0.3 & 0.238 & 0.205 & 0.154 & 0.113 & 0.084 & 0.055 \\
\hline 1.873 & 1.271 & 0.788 & 0.508 & 0.418 & 0.384 & 0.278 & 0.248 & 0.204 & 0.173 & 0.125 & 0.093 & 0.066 & 0.036 \\
\hline 3.099 & 1.794 & 1.116 & 0.765 & 0.646 & 0.576 & 0.435 & 0.414 & 0.38 & 0.341 & 0.265 & 0.173 & 0.098 & 0.059 \\
\hline 1.878 & 1.215 & 0.77 & 0.53 & 0.421 & 0.326 & 0.272 & 0.211 & 0.193 & 0.18 & 0.144 & 0.122 & 0.097 & 0.065 \\
\hline 3.177 & 1.638 & 0.963 & 0.65 & 0.471 & 0.376 & 0.316 & 0.258 & 0.223 & 0.199 & 0.166 & 0.13 & 0.092 & 0.052 \\
\hline 1.717 & 1.133 & 0.705 & 0.493 & 0.448 & 0.346 & 0.292 & 0.261 & 0.246 & 0.222 & 0.163 & 0.127 & 0.1 & 0.063 \\
\hline 1.907 & 1.15 & 0.777 & 0.553 & 0.427 & 0.32 & 0.259 & 0.194 & 0.16 & 0.133 & 0.091 & 0.066 & 0.048 & 0.03 \\
\hline 1.888 & 1.136 & 0.725 & 0.649 & 0.406 & 0.336 & 0.284 & 0.262 & 0.201 & 0.18 & 0.111 & 0.081 & 0.052 & 0.031 \\
\hline 1.87 & 1.37 & 1.033 & 0.628 & 0.486 & 0.414 & 0.338 & 0.27 & 0.235 & 0.207 & 0.161 & 0.124 & 0.095 & 0.056 \\
\hline 1.519 & 1.296 & 0.752 & 0.656 & 0.474 & 0.383 & 0.292 & 0.262 & 0.219 & 0.178 & 0.145 & 0.101 & 0.061 & 0.034 \\
\hline 2.195 & 1.83 & 0.984 & 0.75 & 0.51 & 0.444 & 0.4 & 0.367 & 0.343 & 0.275 & 0.214 & 0.145 & 0.102 & 0.056 \\
\hline 2.337 & 1.4 & 0.988 & 0.722 & 0.568 & 0.494 & 0.448 & 0.342 & 0.274 & 0.242 & 0.157 & 0.124 & 0.081 & 0.054 \\
\hline 2.067 & 0.993 & 0.699 & 0.458 & 0.351 & 0.309 & 0.253 & 0.229 & 0.201 & 0.175 & 0.13 & 0.09 & 0.059 & 0.036 \\
\hline 1.395 & 0.946 & 0.613 & 0.459 & 0.364 & 0.298 & 0.231 & 0.198 & 0.158 & 0.139 & 0.108 & 0.087 & 0.068 & 0.048 \\
\hline 3.178 & 2.012 & 1.161 & 0.821 & 0.597 & 0.494 & 0.413 & 0.368 & 0.329 & 0.272 & 0.207 & 0.169 & 0.12 & 0.075 \\
\hline 2.273 & 1.42 & 0.718 & 0.518 & 0.457 & 0.347 & 0.303 & 0.271 & 0.224 & 0.19 & 0.15 & 0.117 & 0.07 & 0.033 \\
\hline
\end{tabular}

Figure C.1: Fractional durations $\left(T_{n}\right)$ for calibration particle set $\mathrm{B}$, obtained at 0 net pressure on pore A31629. $n$ values are shown in row 1 , each subsequent row relates to different particle. 


\begin{tabular}{|c|c|c|c|c|c|c|c|c|c|c|c|c|c|}
\hline 0.05 & 0.1 & 0.15 & 0.2 & 0.25 & 0.3 & 0.35 & 0.4 & 0.45 & 0.5 & 0.6 & 0.7 & 0.8 & 0.9 \\
\hline 0.454959 & 0.711238 & 062699 & 501502 & 2.020202 & 2.673797 & 3.174603 & 3.636364 & 4.291845 & 1.950495 & 6.493506 & .403361 & 12.5 & 2.72727 \\
\hline & & & & & & & & & & & & & \\
\hline & & & & & & & & & & & & & \\
\hline & & & & & 779 & & & & & & & & \\
\hline & 0.786 & & & & & & & & & & & & \\
\hline & & & & & & & & & & & & & \\
\hline & & & & & & & & & & & & & \\
\hline & & & & & & & & & & & & & \\
\hline & & & & & & & & & & & & 10 & \\
\hline & & & & & & & & & & & & 3333 & \\
\hline & & & & & 619 & 127 & 794 & & & & & & 306 \\
\hline & 27 & & & & 459 & & 04 & & & & 516 & & \\
\hline & & & & & 966 & 4658 & & & & & & & \\
\hline & 0.54 & & & & & 2.5 & & & & & & & 5714 \\
\hline 0.427899 & 0.714286 & D12146 & & 760563 & 024291 & 2.232143 & & & & & .064516 & & 1852 \\
\hline 0.483793 & 1.007049 & 430615 & 183406 & 849003 & 236246 & 3.952569 & 4.366812 & 975124 & & 308 & & & 7778 \\
\hline 0.716846 & 1.057082 & 531321 & 178649 & 253 & 5705 & 004 & 505 & 114 & & 259 & 425 & 588 & 333 \\
\hline 0.314663 & 0.497018 & 861326 & 218027 & & 291 & & & & & & & & \\
\hline 0.439947 & 0.704225 & 392758 & 1.930502 & 2.188184 & 2.881844 & 3.30033 & 3.690037 & 4.464286 & 5.263158 & 6.666667 & 8.547009 & 14.28571 & 30.30303 \\
\hline
\end{tabular}

Figure C.2: Relative velocities $\left(v_{n C a l}^{e l}\right)$ for calibration particle set B, calculated as $1 / T_{n}$ from the values in Figure C.1. $n$ values are shown in row 1, each subsequent row relates to different particle. 


\begin{tabular}{|c|c|c|c|c|c|c|c|c|c|c|c|c|c|}
\hline 0.0 & 0.1 & 0.15 & 0.2 & 0.25 & 0.3 & 0.35 & 0.4 & 0.45 & 0.5 & 0.6 & 0.7 & 0.8 & 0.9 \\
\hline $0.5047 €$ & 872461 & 1.352266 & 1.867744 & 2.409506 & 2.988155 & 3.629947 & 4.356211 & 5.123463 & 5.890523 & 7.684089 & 10.26688 & 4.2785 & 2.88062 \\
\hline
\end{tabular}

Figure C.3: Averaged relative velocities $\left(v_{n C a l}^{e l}\right)$ for calibration particle set B, calculated by averaging the velocities at each $n$ in Figure C.2. $n$ values are shown in row 1. 


\begin{tabular}{|r|r|r|r|r|r|r|r|r|r|r|r|r|r|}
\hline $\mathbf{0 . 0 5}$ & $\mathbf{0 . 1}$ & $\mathbf{0 . 1 5}$ & $\mathbf{0 . 2}$ & $\mathbf{0 . 2 5}$ & $\mathbf{0 . 3}$ & $\mathbf{0 . 3 5}$ & $\mathbf{0 . 4}$ & $\mathbf{0 . 4 5}$ & $\mathbf{0 . 5}$ & $\mathbf{0 . 6}$ & $\mathbf{0 . 7}$ & $\mathbf{0 . 8}$ & $\mathbf{0 . 9}$ \\
\hline 1.18 & 0.976 & 0.753 & 0.437 & 0.372 & 0.357 & 0.217 & 0.188 & 0.17 & 0.156 & 0.129 & 0.098 & 0.079 & 0.057 \\
\hline 1.076 & 0.896 & 0.679 & 0.436 & 0.397 & 0.33 & 0.243 & 0.227 & 0.198 & 0.181 & 0.133 & 0.117 & 0.101 & 0.05 \\
\hline 1.556 & 1.193 & 0.712 & 0.686 & 0.455 & 0.431 & 0.276 & 0.258 & 0.238 & 0.222 & 0.158 & 0.126 & 0.096 & 0.049 \\
\hline 2.791 & 1.78 & 1.355 & 1.161 & 0.97 & 0.841 & 0.634 & 0.614 & 0.577 & 0.542 & 0.448 & 0.113 & 0.077 & 0.043 \\
\hline 2.275 & 1.33 & 1.147 & 0.743 & 0.495 & 0.451 & 0.354 & 0.324 & 0.284 & 0.22 & 0.152 & 0.095 & 0.074 & 0.049 \\
\hline 2.075 & 1.887 & 1.161 & 0.705 & 0.559 & 0.491 & 0.324 & 0.301 & 0.209 & 0.186 & 0.115 & 0.093 & 0.075 & 0.047 \\
\hline 1.058 & 0.696 & 0.494 & 0.381 & 0.318 & 0.291 & 0.28 & 0.201 & 0.133 & 0.1 & 0.074 & 0.053 & 0.036 & 0.022 \\
\hline 1.114 & 0.599 & 0.574 & 0.368 & 0.315 & 0.278 & 0.228 & 0.16 & 0.144 & 0.131 & 0.095 & 0.059 & 0.047 & 0.032 \\
\hline 1.437 & 0.935 & 0.452 & 0.374 & 0.352 & 0.338 & 0.213 & 0.196 & 0.174 & 0.154 & 0.109 & 0.071 & 0.052 & 0.033 \\
\hline 1.619 & 0.959 & 0.731 & 0.64 & 0.505 & 0.377 & 0.275 & 0.261 & 0.246 & 0.206 & 0.176 & 0.137 & 0.096 & 0.079 \\
\hline 0.936 & 0.886 & 0.568 & 0.467 & 0.336 & 0.308 & 0.253 & 0.174 & 0.151 & 0.139 & 0.121 & 0.102 & 0.079 & 0.054 \\
\hline 1.212 & 1.02 & 0.838 & 0.572 & 0.456 & 0.429 & 0.279 & 0.26 & 0.227 & 0.16 & 0.128 & 0.092 & 0.069 & 0.026 \\
\hline 1.735 & 1.197 & 0.978 & 0.516 & 0.39 & 0.363 & 0.255 & 0.237 & 0.216 & 0.156 & 0.125 & 0.095 & 0.063 & 0.044 \\
\hline 2.039 & 1.275 & 0.946 & 0.68 & 0.521 & 0.312 & 0.286 & 0.236 & 0.197 & 0.187 & 0.156 & 0.097 & 0.072 & 0.05 \\
\hline 1.788 & 0.878 & 0.5 & 0.479 & 0.355 & 0.339 & 0.218 & 0.202 & 0.144 & 0.127 & 0.095 & 0.067 & 0.048 & 0.029 \\
\hline 1.909 & 1.141 & 0.924 & 0.582 & 0.376 & 0.362 & 0.348 & 0.274 & 0.238 & 0.218 & 0.19 & 0.158 & 0.133 & 0.081 \\
\hline 2.634 & 1.79 & 1.509 & 0.713 & 0.669 & 0.438 & 0.408 & 0.304 & 0.192 & 0.168 & 0.107 & 0.082 & 0.056 & 0.035 \\
\hline 3.99 & 3.284 & 2.573 & 2.144 & 0.678 & 0.539 & 0.436 & 0.382 & 0.298 & 0.238 & 0.192 & 0.141 & 0.11 & 0.073 \\
\hline 3.456 & 1.498 & 1.359 & 1.176 & 0.9 & 0.759 & 0.61 & 0.585 & 0.516 & 0.498 & 0.418 & 0.389 & 0.253 & 0.128 \\
\hline
\end{tabular}

Figure C.4: Fractional durations $\left(T_{n}\right)$ for sample particle set $D$, obtained at 0 net pressure on pore A31629. $n$ values are shown in row 1 , each subsequent row relates to different particle. 


\begin{tabular}{|c|c|c|c|c|c|c|c|c|c|c|c|c|c|}
\hline 0.05 & 0.1 & 0.15 & 0.2 & 0.25 & 0.3 & 0.35 & 0.4 & 0.45 & 0.5 & 0.6 & 0.7 & 0.8 & 0.9 \\
\hline 47458 & 2459 & 021 & & 172 & 30112 & 608295 & 319149 & 53 & 6.410256 & 7.751938 & 5408 & 12.65 & 17.54386 \\
\hline 368 & 116071 & 754 & 5578 & 518892 & 30303 & 115226 & 186 & 050 & 4862 & 7.518797 & 547009 & 90099 & 20 \\
\hline 0.642674 & 0.838223 & 1.404494 & 1.457726 & 2.197802 & 2.320186 & 3.623188 & 3.875969 & .201681 & 4.504505 & 6.329114 & 7.936508 & 10.41667 & 20.40816 \\
\hline 0.358295 & 0.561798 & 0.738007 & 0.861326 & 1.030928 & 1.189061 & 1.577287 & 1.628664 & 1.733102 & 1.845018 & 2.232143 & 8.849558 & 12.98701 & 23.25581 \\
\hline 0.43956 & 75188 & 0.87184 & 1.345895 & 2.020202 & 2.217295 & 2.824859 & 3.08642 & 3.521127 & 4.545455 & 6.578947 & 10.52632 & 13.51351 & 20.40816 \\
\hline 0.481928 & 0.529942 & 0.861326 & 1.41844 & 1.788909 & 2.03666 & 642 & 3.322259 & 4.784689 & 5.376344 & 5652 & 269 & 13.33333 & 21.2766 \\
\hline 0.94518 & 1.436 & 291 & 2.624672 & 3.14 & 3.43 & 3.57 & 4.975124 & 7.518797 & 10 & & 5792 & 7778 & 45.45455 \\
\hline 0.897666 & 1.669449 & 1.74216 & 2.717391 & 3.174603 & 3.597122 & 4.38 & 6.25 & 6.944444 & 7.633588 & 10.52632 & 1915 & 21.2766 & 31.25 \\
\hline 0.695894 & 1.069519 & 2.212389 & 2.673797 & 2.840909 & 2.95858 & 4.694836 & 5.102041 & 5.747126 & 6.493506 & 9.174312 & 8451 & 19.23077 & 30.30303 \\
\hline 0.617665 & 1.042753 & 989 & 625 & 1.980198 & 2.65252 & 364 & 3.831418 & 4.06 & 4.854369 & 1818 & 9927 & 10.41667 & 12.65823 \\
\hline 1.068376 & 1.128668 & 1.760563 & 2.141328 & 2.97619 & 3.246753 & 3.95 & 5.747126 & 6.622517 & 7.194245 & 8.264463 & 3922 & 55823 & 18.51852 \\
\hline 0.825083 & 0.980392 & 1.193317 & 1.748252 & 2.192982 & 2.331002 & 3.584229 & 3.846154 & 4.405286 & 6.25 & 7.8125 & 10.86957 & 14.49275 & 38.46154 \\
\hline 0.576369 & 0.835422 & 1.022495 & 984 & 2.564103 & 2.754821 & 1569 & 4.219409 & 4.62963 & 6.410256 & & 10.52632 & 15.87302 & 22.72727 \\
\hline & 0.78 & 1.057082 & 1.47 & & 3.2 & & & & & & & 889 & 20 \\
\hline & 1.13 & & 2.08 & 2.81 & 2.94 & & 4.9 & 6.9 & 7.87 & & 14. & 333 & 34.48276 \\
\hline & 0.876424 & 2251 & 1.71 & 2.65 & 2.762431 & 2.8 & 3.6 & & 7156 & & & 797 & 12.34568 \\
\hline 651 & 0.558659 & 0.662691 & 1.402525 & 1.494768 & 2.283105 & 5098 & 39474 & 5.2 & 52381 & 5794 & 19512 & 5714 & 28.57143 \\
\hline 0627 & 0.304507 & 0.388651 & 0.466418 & 1.474926 & 1.855288 & 3578 & 2.617801 & 5705 & 4.201681 & 88333 & 7.092199 & 0909 & 13.69863 \\
\hline 0.289352 & 0.667557 & 0.735835 & 0.85034 & 1.111111 & 1.317523 & 1.639344 & 1.709402 & 1.937984 & 2.008032 & 2.392344 & 2.570694 & 3.952569 & 7.8125 \\
\hline
\end{tabular}

Figure C.5: Relative velocities ( $v_{n \text { Sample }}^{e l}$ ) for sample particle set D, calculated by averaging the velocities at each $n$ in Figure C.4. $n$ values are shown in row 1, each subsequent row relates to different particle. 


\begin{tabular}{|c|c|c|c|c|c|c|c|c|c|c|c|c|c|}
\hline 0.05 & 0.1 & 0.15 & 0.2 & 0.25 & 0.3 & 0.35 & 0.4 & 0.45 & 0.5 & 0.6 & 0.7 & 0.8 & 0.9 \\
\hline 1.6789 & 1.174368 & & 225184 & 653 & & 269521 & 1.221049 & 14812 & .088232 & 1.00883 & & & \\
\hline & & & & & & & & & & & & & \\
\hline & & & & & & & & & & & & & \\
\hline & & & & & & & & & & & & & \\
\hline & & & & & & & & & & & & & 941 \\
\hline & & & & & & 266 & & & & & & & 896 \\
\hline & & & & & & 879 & 076 & & & & & 427 & 5596 \\
\hline & & & & & & 273 & 733 & & & 885 & & 115 & 784 \\
\hline & 1.22 & & & & & 362 & 211 & & & & & 834 & 4397 \\
\hline & & & & & & & & & & & & & 3229 \\
\hline & & & & & & & & & & & 4907 & 524 & 9354 \\
\hline 1.634573 & 1.123709 & 0.882458 & 0.936023 & & 0.780081 & 0.987406 & 913 & 9826 & & & 8702 & 006 & 0965 \\
\hline 1.141846 & 0.957546 & 0.756134 & 1.037607 & 064161 & 0.921914 & 1.080338 & 0.968596 & 0.903613 & & & 5269 & 1673 & 3298 \\
\hline 0.971605 & 0.898967 & 0.781712 & 0.787361 & 0.796589 & 1.072611 & 0.963238 & 0.9727 & 0.990764 & & 0.834225 & 4129 & 2714 & 4102 \\
\hline 1.107999 & 1.305448 & 999 & 1.11 & 078 & 0.98 & 3698 & 1.136422 & & & & 5374 & 0071 & 1.507072 \\
\hline 777 & & & & & & 627 & 3378 & & & & 459 & & 9569 \\
\hline & 26 & & & & & & & & & & & & 3717 \\
\hline & & & & & & & & & & & & & 987 \\
\hline 0.573236 & 0.765142 & & & & & 0.451617 & & & & 0.311337 & 0.250387 & 0.27682 & 0.341446 \\
\hline
\end{tabular}

Figure C.6: Ratio of the relative velocities of sample particle set D over the averaged relative velocities of calibration particle set B $\left(v_{n S a m p l e}^{e l} / v_{n C a l}^{e l}\right)$ taken from Figures C.5 and C.3 respectively. $n$ values are shown in row 1 , each subsequent row relates to different sample particle. 


\begin{tabular}{|c|c|c|c|c|c|c|c|c|c|c|c|c|c|}
\hline 0.05 & 0.1 & 0.15 & 0.2 & 0.25 & 0.3 & 0.35 & 0.4 & 0.45 & 0.5 & 0.6 & 0.7 & 0.8 & 0.9 \\
\hline-35.73946 & -29.98779 & -27.79561 & -30.5671 & -29.31844 & -27.28645 & -31.07254 & -30.51996 & -29.68857 & -29.00585 & -28.10066 & -27.93027 & -26.70637 & -25.34102 \\
\hline-37.58937 & -31.18313 & -29.01575 & -30.59913 & -28.51753 & -28.1608 & -29.52404 & -28.12843 & -27.83766 & -27.29233 & -27.75478 & -26.09031 & -24.50498 & -26.56476 \\
\hline-31.1145 & -27.55263 & -28.4403 & -25.49741 & -26.99837 & -25.45165 & -27.97878 & -26.74323 & -25.94898 & -25.31762 & -25.98978 & -25.41243 & -24.9167 & -26.76813 \\
\hline-24.69193 & -23.94072 & -22.82162 & -21.85721 & -21.47759 & -21.13634 & -21.55354 & -20.86214 & -20.45625 & -20.17069 & -19.91157 & -26.42625 & -26.96888 & -28.18693 \\
\hline-26.52728 & -26.42443 & -23.94986 & -24.81483 & -26.1581 & -25.05912 & -25.47159 & -24.67702 & -24.43471 & -25.39687 & -26.36043 & -28.28807 & -27.38923 & -26.76813 \\
\hline-27.48413 & -23.52448 & -23.86124 & -25.25762 & -25.06379 & -24.36999 & -26.29303 & -25.2942 & -27.24621 & -27.0049 & -29.50074 & -28.53942 & -27.24538 & -27.20081 \\
\hline-37.94647 & -35.37369 & -33.66537 & -32.62 & -31.47818 & -29.71018 & -27.81622 & -29.61967 & -33.32976 & -35.95312 & -36.64845 & -37.55031 & -38.77787 & -39.24719 \\
\hline-36.8734 & -38.41383 & -31.28692 & -33.18593 & \begin{tabular}{|l|}
-31.61987 \\
\end{tabular} & -30.32325 & -30.37431 & -32.95596 & -32.05179 & -31.37338 & -32.21669 & -35.41977 & -33.58731 & -32.16994 \\
\hline-32.31647 & -30.57485 & -35.25109 & -32.91984 & -30.04108 & -27.88717 & -31.34433 & -29.9518 & -29.38769 & -29.16696 & -30.21087 & -32.23896 & -31.95391 & -31.69813 \\
\hline-30.5497 & -30.22512 & -28.13255 & -26.13691 & -25.96883 & -26.71953 & -28.02015 & -26.62664 & -25.64495 & -25.99472 & -25.02946 & -24.70486 & -24.9167 & -22.90681 \\
\hline-40.72881 & -31.34773 & -31.44207 & -29.66985 & -30.68113 & -28.98657 & -29.01321 & -31.63996 & -31.33548 & -30.52311 & -28.86103 & -27.48594 & -26.70637 & -25.82663 \\
\hline-35.23413 & -29.41028 & -26.66002 & -27.27067 & -26.97557 & -25.49292 & -27.85642 & -26.6652 & -26.40202 & -28.6957 & -28.19051 & -28.6692 & -28.17106 & -35.76301 \\
\hline-29.61704 & -27.51603 & -25.21993 & -28.42872 & -28.73144 & -27.10982 & -28.91585 & -27.642 & -26.90119 & -29.00585 & -28.46868 & -28.28807 & -29.27307 & -27.92359 \\
\hline-27.6763 & -26.84822 & -25.51152 & -25.57591 & -25.68111 & -28.82777 & -27.58092 & -27.68878 & -27.89471 & -26.94926 & -26.11016 & -28.04708 & -27.68894 & -26.56476 \\
\hline-29.23119 & -31.4821 & -33.46059 & -29.34242 & -29.92749 & -27.85387 & -31.00616 & -29.55521 & -32.05179 & -31.83868 & -32.21669 & -33.17263 & -33.2334 & -33.78063 \\
\hline-28.43057 & -28.05178 & -25.7237 & -27.08732 & -29.18314 & -27.13885 & -25.62455 & -26.15092 & -25.94898 & -25.47758 & -24.40834 & -23.62763 & -22.60303 & -22.75109 \\
\hline-25.17425 & -23.89971 & -22.18668 & -25.16048 & -23.67214 & -25.31019 & -24.29741 & -25.2084 & -28.18884 & -28.11972 & -30.46528 & -30.14105 & -30.8572 & -30.83538 \\
\hline-22.26029 & -20.57883 & -19.87645 & -19.44684 & -23.57826 & -23.67804 & -23.80308 & -23.45066 & -24.06664 & -24.73156 & -24.32701 & -24.47494 & -23.85821 & -23.42518 \\
\hline
\end{tabular}

Figure C.7: $\zeta_{n \text { Sample }}$ of sample particle set D calculated using the ratios in Figure C.6 in combination with calibration $\zeta_{C a l}$ and $\zeta_{\text {pore }}$ values using Equation 4.12. 